
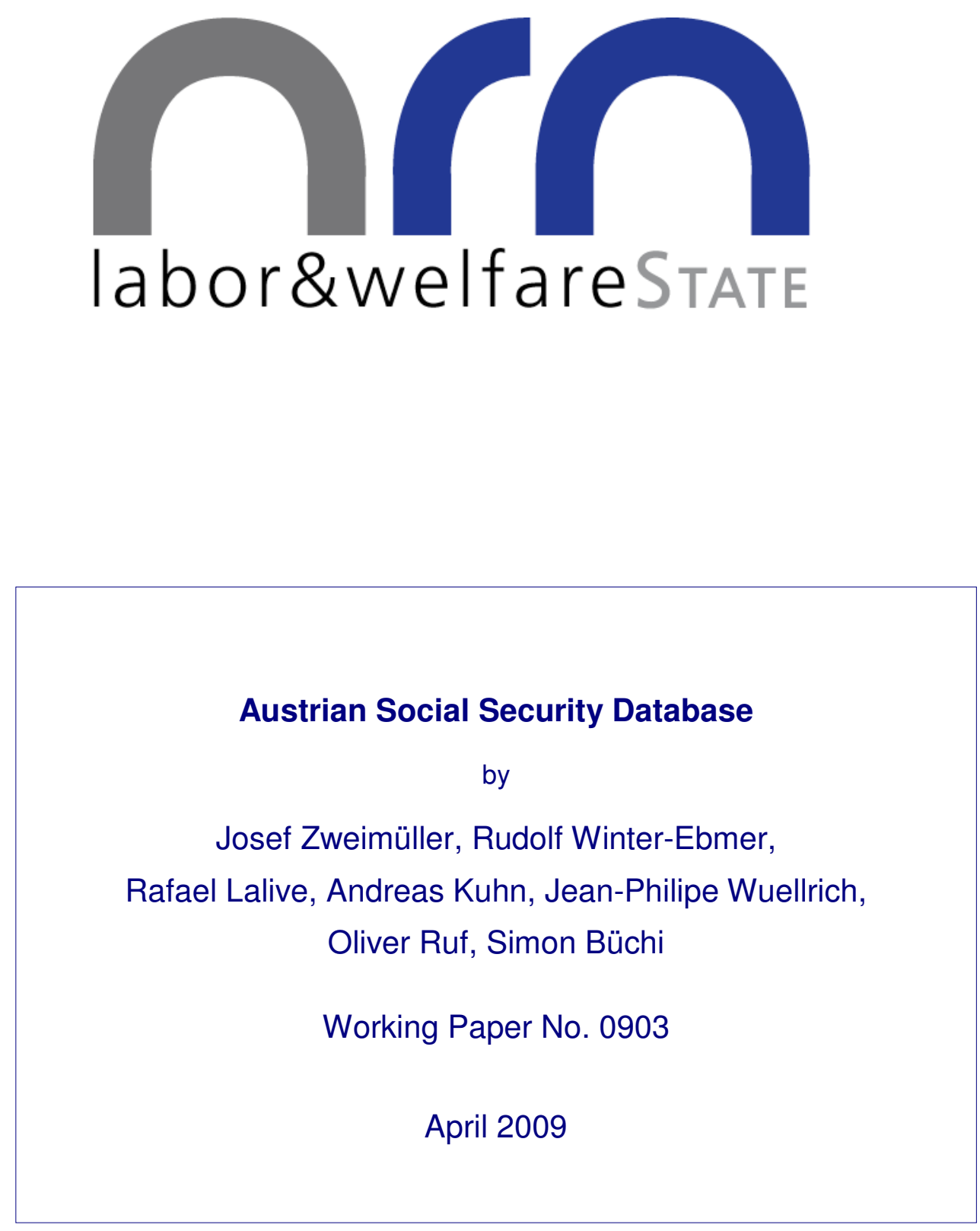

Supported by the Austrian Science Funds



The Austrian Center for Labor Economics and the Analysis of the Welfare State

JKU Linz

Department of Economics

Altenberger Strasse 69

4040 Linz, Austria

www.labornrn.at 


\title{
Austrian Social Security Database
}

\author{
Josef Zweimüller \\ Rudolf Winter-Ebmer \\ Rafael Lalive \\ Andreas Kuhn \\ Jean-Philipe Wuellrich \\ Oliver Ruf \\ Simon Büchi * \\ University of Zurich \\ University of Linz \\ University of Lausanne \\ SUVA Luzern
}

April 21, 2009

\begin{abstract}
The Austrian Social Security Database (ASSD) is a matched firm-worker data set, which records the labor market history of almost 11 million individuals from January 1972 to April 2007. Moreover, more than 2.2 million firms can be identified. The individual labor market histories are described in the following dimensions: very detailed daily labor market states and yearly earnings at the firm-worker level, together with a limited set of demographic characteristics. Additionally the ASSD provides some firm related information, such as geographical location and industry affiliation. This paper is a short description of this huge data base and intended for people using this data in their own empirical work.
\end{abstract}

*Address: Josef Zweimüller (zweim@iew.uzh.ch), Andreas Kuhn (kuhn@iew.uzh.ch), JeanPhilippe Wuellrich (wuellrich@iew.uzh.ch), Simon Büchi (sibuechi@iew.uzh.ch), Institute for Empirical Research in Economics, Bluemlisalpstr. 10, 8006 Zurich, Switzerland; Rudolf Winter-Ebmer (Rudolf.WinterEbmer@jku.at), Department of Economics, University of Linz, 4040 Linz, Austria; Rafael Lalive, University of Lausanne, Faculty of Business and Economics, Extranef 210, 1015 Lausanne, Switzerland; Oliver Ruf (oliver.ruf@suva.ch), Suva, Postfach 4358, Fluhmattstrasse 1, 6002 Luzern, Switzerland 


\section{Contents}

\begin{tabular}{lll}
\hline 1 & Introduction & 4
\end{tabular}

\begin{tabular}{|ll|}
\hline 2 & Labor market history 1972-2007
\end{tabular}

$2.1 \quad$ Labor market states (qual_i.dta, $i \in\{1,2, \ldots, 40\}$ ) . . . . . . . . 5

2.2 Yearly Earnings (bg- $j$. dta, $j \in\{1,2, \ldots, 40\}$ or $j \in\{1972, \ldots, 2007\})$. . 6

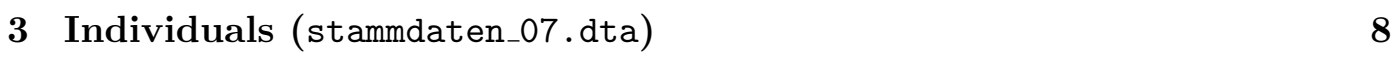

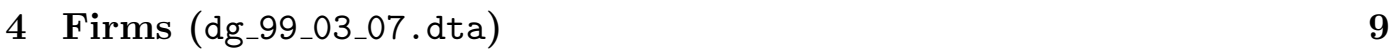

\begin{tabular}{|lll}
5 & Labor Market History before 1972 (REV data) & 10
\end{tabular}

5.1 Overview . . . . . . . . . . . . . . . . . . 10

5.2 Introduction . . . . . . . . . . . . . . . . . 10

5.3 Who is in the REV data? . . . . . . . . . . . . . . . 11

\begin{tabular}{|ll}
\hline A Script "normspell2.do" & 13
\end{tabular}

\begin{tabular}{ll}
\hline B Figures & 15
\end{tabular}

\begin{tabular}{lr}
\hline C Tables & 17
\end{tabular} 


\section{List of Figures}

1 Geographical representation of the nuts-classification . . . . . . . . . 15

$2 \quad$ Employees of 1972 and fraction in REV data . . . . . . . . . . . 16

3 Employees of 1972 working in 1985 (or afterwards) and fraction in REV data . . . . . . . . . . . . . . . . . 16

\section{List of Tables}

$1 \quad$ Detailed description of the different types of qualifications . . . . . . 17

$2 \quad$ Labor market states: classification . . . . . . . . . . . . . . . 36

$3 \quad$ Dates at which qualifications are first introduced . . . . . . . . . . . . 37

$4 \quad$ Minimal and maximal contribution basis, 1972-2007, in $€$. . . . . . . 42

5 Codelist for the variable stsl . . . . . . . . . . . . . . . . . . . . . . 43

$6 \quad$ Codelist for the variable bundesland $\ldots \ldots$. . . . . . . . . . . . . . . 49

$7 \quad$ Codelist for the variable beot $\ldots \ldots \ldots$. . . . . . . . . . 50

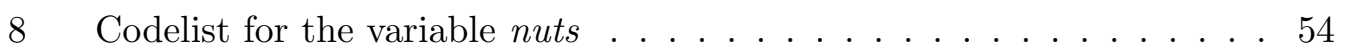

$9 \quad$ Codelist for the variable industry_15 . . . . . . . . . . . . . 56

10 Codelist for the variable wikl_25 . . . . . . . . . . . . . . . 57

11 Codelist for the variable wikl 59 . . . . . . . . . . . . . . 58

12 Codelist for the variable onace . . . . . . . . . . . . . . . . 60

13 Codelist for the variable onace_17 f . . . . . . . . . . . . . 78

14 Codelist for the variable onace_60 . . . . . . . . . . . . . . . . 79 


\section{Introduction}

Austrian Social Security is organized in a decentralized structure and comprises Health, Accident and Pension Insurance. Responsibility for social security is delegated to independent bodies, the insurance institutions. There are 23 insurers 16 providing health insurance and 7 general insurance institutions - some of which are also responsible for two or even all three types of social security. For historical reasons the system is structured on a geographical as well as occupational basis. The Main Association of Austrian Social Security Institutions (Hauptverband der österreichischen Sozialversicherungsträger) administers the ASSD by gathering the individual data of each of the 23 Austrian social security institutions. The Main Association of Austrian Social Security Institutions has been collecting data using computerized support since 1972. This is why January 1, 1972 consitutes the "natural" beginning of the ASSD. Social security information prior to that date has not been thoroughly digitized.

\section{$2 \quad$ Labor market history $1972-2007$}

The ASSD records the universe of spells of individuals, which determine the eligibility for and the amount of their social security benefits regarding health, accident, and pension insurance. There are more than 200 different types of spells that contribute to that determination (we refer to these types as qualifications in the following). These qualifications can be translated into labor market states (see section 2.1 for details). The determination of the amount of the social security benefits additionally depends on social security contributions, which are derived from yearly individual earnings. Accordingly, the ASSD contains (imperfect) information on yearly earnings (see section 2.2 for details). 


\subsection{Labor market states (qual_i.dta, $i \in\{1,2, \ldots, 40\}$ )}

The labor market states are archived in forty different files named qual_i.dta $(i \in$ $\{1,2, \ldots, 40\})$, each of which contains approximately a 2.5 percent random sample of the pool of all individuals (i.e. each file contains the universe of spells for roughly 275,000 individuals). The observation unit in this data is a spell indicating in what time period, i.e. from (vdat) and until (bdat) when, an individual (svnr, the "social security number") was subject to an activity (qual) relevant for social security benefits and with whom (benr) this activity is associated. Whether or not the spell is associated with a firm is indicated by $k t k z$. If $k t k z=\mathrm{K}$, then benr contains the firm identifier (firm number). If $k t k z=\mathrm{F}$, then benr contains an identifier for the social security institution (e.g. if a spell concerns unemployment, retirement, etc.). The data is structured as follows:

\begin{tabular}{lll} 
variable & description & code \\
\hline svnr & social security number & - \\
benr & firm number & - \\
ktkz & spell association & $\mathrm{K}=$ firm \\
& & $\mathrm{F}=$ social security institution \\
vdat & start of spell & Stata time format \\
bdat & end of spell & Stata time format \\
qual & qualification & see table 1 in the appendix \\
svnrklasse & file number $i$ & $i \in\{1,2, \ldots, 40\}$
\end{tabular}

Notes: ${ }^{\star}$ left-censored if start date before January 1, 1972. ${ }^{\star \star}$ contains missing values if spell is right-censored

Since there are more than 200 different types of qualifications, they need to be converted into a smaller and economically meaningful classifications, i.e. into labor market states. Unfortunately, there are some qualifications that cannot be unambiguously translated into a labor market state 1 Table 2 provides an example of such a classification. The following main categories are defined: (i) employed, (ii) unemployed, (iii) retired, (iv) sick, (v) special interest, and (vi) other. Note, however, that depending on your own research question, you may want to use your own classification 2

There is a practical problem arising if you want to assign an unambiguous labor market state to an individual at a specific reference date or if you want, for example, determine the labor market experience for an individual: the spells may overlap,

\footnotetext{
${ }^{1}$ For example qualification 48 (Sonderunterstützung) is somewhere between unemployment and retirement.

${ }^{2}$ For example, short sickness leaves are classified a sickness according to our classification, although in judicial terms the employment contract is not interrupted. Thus you may want to reclassify these qualifications as employment.
} 
meaning that an individual may have more than one qualification at once. To handle this problem, we provide the following Stata script (see section A for the stata code): normspel12.do. To run the script, you have to specify four arguments: byvar, start, end and gap. The first argument specifies that you wish to correct the spells within this variable or set of variables. The second and third argument specify the variables containing the starting and end of a spell (i.e. vdat and bdat). The last argument is used for collapsing spells, which have a gap between them no more than specified by this argument. The Stata command is as follows (this would eliminate any overlapping spells at the "svnr benr"-level and at the same time eliminate all gaps between spells at the "svnr benr"-level of 30 or less days):

do .../normspel12. do ' 'svnr benr', vdat bdat 30

Another issue to pay attention to are qualifications that were only introduced after 1972. For example, civil servants were only included in the ASSD since 1988. Consequently, this results in inconsistencies, when, for instance, you want to calculate employment rates from 1972-2007. Table 3 displays for each qualification the date at which it has appeared for the first time in the ASSD.

\subsection{Yearly Earnings (bg $j$.dta, $j \in\{1,2, \ldots, 40\}$ or $j \in\{1972, \ldots, 2007\}$ )}

The contribution basis, according to which social security benefits are calculated, is directly linked to the yearly earnings of an individual. However, the contribution basis features lower and upper boundaries, which means that we do not observe earnings for the lower tail of the earnings distribution and we only observe the upper boundary of the contribution basis for the upper tail of the earnings distribution (i.e. right-censoring). Note that the values of the lower and upper boundaries vary over time. Table 4 displays these values. Furthermore, the yearly earnings are collected at the "svnr benr"-level, i.e. we observe the yearly earnings of an individual for each different firm within a year. The social security contributions are edited in two different forms. You can use either form - depending on how you want to use the earnings information. The yearly earnings are split up into two parts: (i) the contribution basis in $€($ bg_euro), and (ii) special payments in $€$ (sz_euro). The former is the yearly wage and the latter part contains supplementary payments such as 13 th, or 14 th monthly wage, christmas bonus or extra vacation payments. In order to calculate the daily wage, we need to know how many days an individual has 
worked for a firm a year. This information is contained in (duration), which sums up all (non-overlapping) spell lengths at the "svnr benr"-level in a given year that are associated with a firm (i.e. $k t k z=\mathrm{K}$ ). The daily wage (dailywage) is calculated by adding up the yearly wage and the supplementary payments and dividing this amount by duration.

The observation unit in this data are yearly earnings of a "svnr benr"-pair in a given year (1972-2007). The data is structured as follows:

\begin{tabular}{llc} 
variable & description & code \\
\hline svnr & social security number & - \\
benr & firm number & - \\
contryear & year of contribution & - \\
bg_euro & bg in $€($ contribution basis) & - \\
sz_euro & special payments in $€$ & $\mathrm{~K}=$ firm \\
& (e.g. bonuses) & $\mathrm{F}=$ social security institution \\
ktkz & spell association & - \\
& & $j \in\{1,2, \ldots, 40\}$ \\
tetg & non-informative variable & - \\
svnrklasse & file number $j^{\star}$ & - \\
duration & sum of the number of days from each spells & - \\
& within a year associated with a specific firm & \\
dailywage & daily wage & \\
\hline
\end{tabular}

Notes: ${ }^{\star}$ same allocation as in the qualification files. ${ }^{\star \star}$ adjusted for overlapping spells. $\star \star \star=\frac{\text { contribution basis }+ \text { special payments }}{\text { duration }}$ 


\section{Individuals (stammdaten_07.dta)}

The individual level data contain some basic demographic information. Note that this is time invariant date, i.e. this is the state of an individual at the time of the data delivery (April 2007). The data structure is as follows:

\begin{tabular}{|c|c|c|}
\hline variable & description & code \\
\hline Svnr & \multicolumn{2}{|l|}{ social security number - } \\
\hline frau & sex & $\begin{array}{l}1=\text { female } \\
0=\text { male }\end{array}$ \\
\hline birhtyear & year of birth & - \\
\hline birthmonth & month of birth & - \\
\hline \multirow[t]{10}{*}{ akgr } & \multirow[t]{10}{*}{ academic degree $^{\star}$} & $0=$ no academic degree \\
\hline & & $1=$ Doktor $($ Dr. $)$ \\
\hline & & $2=$ Magister (Mag.) \\
\hline & & $3=$ Diplomingenieur (Dipl.Ing.) \\
\hline & & $4=$ Diplomkaufmann (DKfm) \\
\hline & & 5 = Diplomvolkswirt (Dipl.Vw.) \\
\hline & & $6=$ Tierarzt $($ Tzt $)$ \\
\hline & & $7=$ Diplomdolmetsch (Dipl.Dolm.) \\
\hline & & $8=$ Diplomtierarzt (Dipl.Tzt) \\
\hline & & $9=$ Ingenieur (Ing.) \\
\hline & nationality & see table 5 \\
\hline stsl_former & nationality in 1999 & see table斉 \\
\hline deathday & day of death & - \\
\hline deathmonth & month of death & - \\
\hline deathyear & year of death & - \\
\hline \multirow[t]{3}{*}{ stma } & specifying death date & $\begin{array}{l}1=\text { date of death refers to the date when the person } \\
\text { died (day, month, and year known) }\end{array}$ \\
\hline & & $\begin{array}{l}2=\text { date of death refers to the date when the death } \\
\text { was notified (only month and year known) }\end{array}$ \\
\hline & & $\begin{array}{l}3=\text { person is dead (but either date unknown: day } \\
\text { contains missing values, month and year contain zeros) }\end{array}$ \\
\hline svnrklasse & qual/bg - file number & $\in\{1,2, \ldots, 40\}$ \\
\hline
\end{tabular}

Notes: ${ }^{\star}$ only those which are compulsory to carry from law. 


\section{Firms (dg_99_03_07.dta)}

The firm level data contain some information related to the geographical location as well as the industry affiliation. Note that this is time invariant date, i.e. this is the state of a firm at the time of the data delivery (i.e. 1999, 2003, or 2007, which is indicated by data_delivery). The data structure is as follows:

\begin{tabular}{|c|c|c|}
\hline variable & description & code \\
\hline benr & firm number & - \\
\hline vstr & non-informative variable & - \\
\hline data_delivery & year of data delivery & 1999,2003 , or 2007 \\
\hline formation & year of firm number assignment & - \\
\hline bundesland & province & see table bundesland \\
\hline beot & firm location (6 digits) & see table 7 \\
\hline plzl & zip code & - \\
\hline nuts & firm location (EU compliant) & see table 8 and figure 1 \\
\hline industry_15 * & industry affiliation (15 cat.) & \\
\hline wikl_25 & industry affiliation according to wikl (25 cat.) & see table 10 \\
\hline wikl_59 & industry affiliation according to wikl (59 cat.) & see table $\overline{11}$ \\
\hline onace & original onace code (4 digits) & see table $\overline{12}$ \\
\hline onace_17 & industry affiliation according to onace (17 cat.) & see table $\overline{\overline{13}}$ \\
\hline onace_60 & industry affiliation according to onace (60 cat.) & see table $\overline{14}$ \\
\hline
\end{tabular}

Notes: * bases on wikl_25 and replaces missing values by information from onace_60 - if not missing either. 


\section{Labor Market History before 1972 (REV data)}

\subsection{Overview}

The REV data is an extension to the Austrian Social Security Database (ASSD) providing social security information prior to January 1972. Despite a very similar data structure, some important differences exist between the two sources. Especially, the REV data does not contain any firm information, and it does not represent an equally comprehensive sample of the labor force. For practical purposes, information from the REV data has shown to be reliable for individuals who are still economically active after the mid-1980s.

\subsection{Introduction}

Social security information prior to 1972 has not been thoroughly digitized. In the early 1980s, however, the Main Association of Social Security Institutions established a file where a considerable part of the missing records has been registered in electronic form. In case older information of a person was needed, i.e. in the event of an application for a pension or if an extract of the current insurance time was required by the insured person, all information of this individual was incorporated in the so called REV data ( $R E V$ stands for $\boldsymbol{R}$ ückwirkend $\boldsymbol{E}$ rfasste $\boldsymbol{V}$ ersicherungsdaten).

Data is stored almost identically to the ASSD, but some of its contents is missing. As a main difference, no firm identifiers are attributed to employment spells, so the REV data can not be used to track individual working histories in detail.

rev_stammdaten.dta is the equivalent to the ASSD-file of the same name (see section 3), sharing the same variables but birthmonth which is not included in the REV data. A missing svnrklasse variable indicates that the corresponding person is not available in the ASSD.

rev_qualifikation.dta contains the same qualification spells as the ASSD (see section 2.1]. As pointed out above, no firm numbers are present. The original $k t k z$ is not available, too, what is the reason for the newly constructed $k t k z_{-} q u a l 0 \%$. To each qualification, the ktkz indication of the ASSD data delivery 2007 was assigned, thus defining the value of the new variable. The assignment was unique for each qualification type. Missing values belong to (usually World War II related) qualifications not existing in the ASSD. 
rev_beitragsgrundlagen.dta stores the contribution basis of the individuals in Euros. duration corresponds to the total duration of ktkz-qual07 = "K" spells of a given year. In contrast to the ASSD (see section 2.2), due to the missing firm information the variable value results from spells of all employers. dailywage equals the sum of bg_euro and sz_euro divided by duration. Since some wage information stem from fictious firms $\left(k t k z_{-} q u a l 07=\right.$ "F"), the $d u$ ration and dailywage variables may not be comparable to the corresponding ASSD values. Moreover, for many employment spells we do not observe any contribution payments, and disproportionately often contributions are set to zero. It is therefore absolutely not recommended to use wage information from this file without investigating further.

\subsection{Who is in the REV data?}

In the REV data, pre-1972 social security security information of 2,379,724 persons is stored. Individual records have been included by the administrators if they were needed after some point in time in the late 1970s. More precisely, people can be assumed to be in the REV data if an assessment of the insurance time was required after that date. This may be provoked by any reason, but it occurs primarily in the case of a pension claim. Unfortunately, the start of the adaption of this practice is not known. However, data allows some inference.

Figure 2 plots the number of people working in 1972 and the fraction with a REV data - entry for each year of birth. We can expect most of the workforce of 1972 to have worked already in 1971 and before, so a high fraction indicates an overall good availability of ASSD individuals in the REV data. The graphs show that the matching quality jumps remarkably for men born in 1919 and women born in 1924 . Since individuals are usually included at the time they retire and since typical pension ages are at about 60 (men) and 55 (women), we can expect the data recording to have started around 1979. Matching quality further improves with younger cohorts. On the other hand, quality deteriorates with year of birth of about 1950 (men) / 1955 (women) and younger. This can be easily explained by the fact that a notable part of these individuals had not yet attained the age of retirement when the ASSD ends in 2007. Another bunch of people may have started their working career in 1972, so they are not in the REV data for obvious reasons.

Given this overall picture, a high fraction of positive matches is likely to occur for 
people not retired in the mid-1980s but who once became eligible for a pension benefit. A person subgroup presumably fulfilling these criteria are those individuals who were working in 1972 and still on the job in the mid-1980s ${ }^{3}$ This is verified in figure 3 which reprints figure 2 for this new sample.

Again, the matching rate declines with younger cohorts for the reasons already explained. With regard to their age in 1972, however, relatively little information is lost compared to the missing social security records of older people.

In summary, the following members of the pre-1972 workforce are usually not included in the REV data:

- Retirees and the deceased of the early 1980s and before

- Individuals with little work experience (no eligibility for a pension)

- People not yet retired in early 2007. E.g. for a men who started working at the age of 18 and intends to retire in 2008 at the regular pension age of $65^{4}$. this implies a missing social insurance contribution span of 11 years. Although an extreme case, this situation is not unrealistic. A careful sample selection is essential to prevent such gaps when REV data is used.

\footnotetext{
${ }^{3}$ Retirement candidacy requires relatively long working histories.

${ }^{4}$ Year of birth $=1943$.
} 


\section{A Script "normspell2.do"}






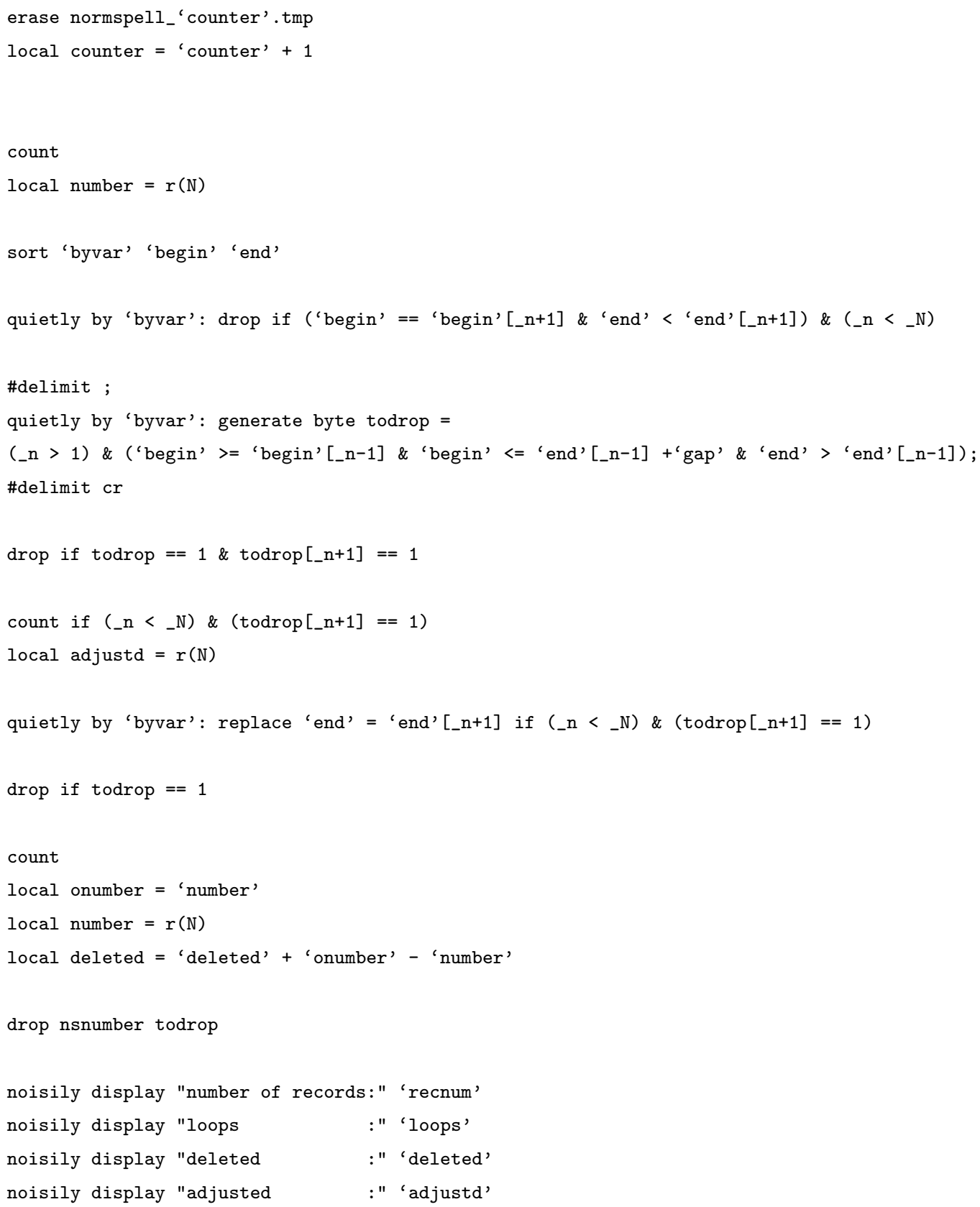


B Figures

Gliederung in NUTS I- und NUTS-II-Einheiten

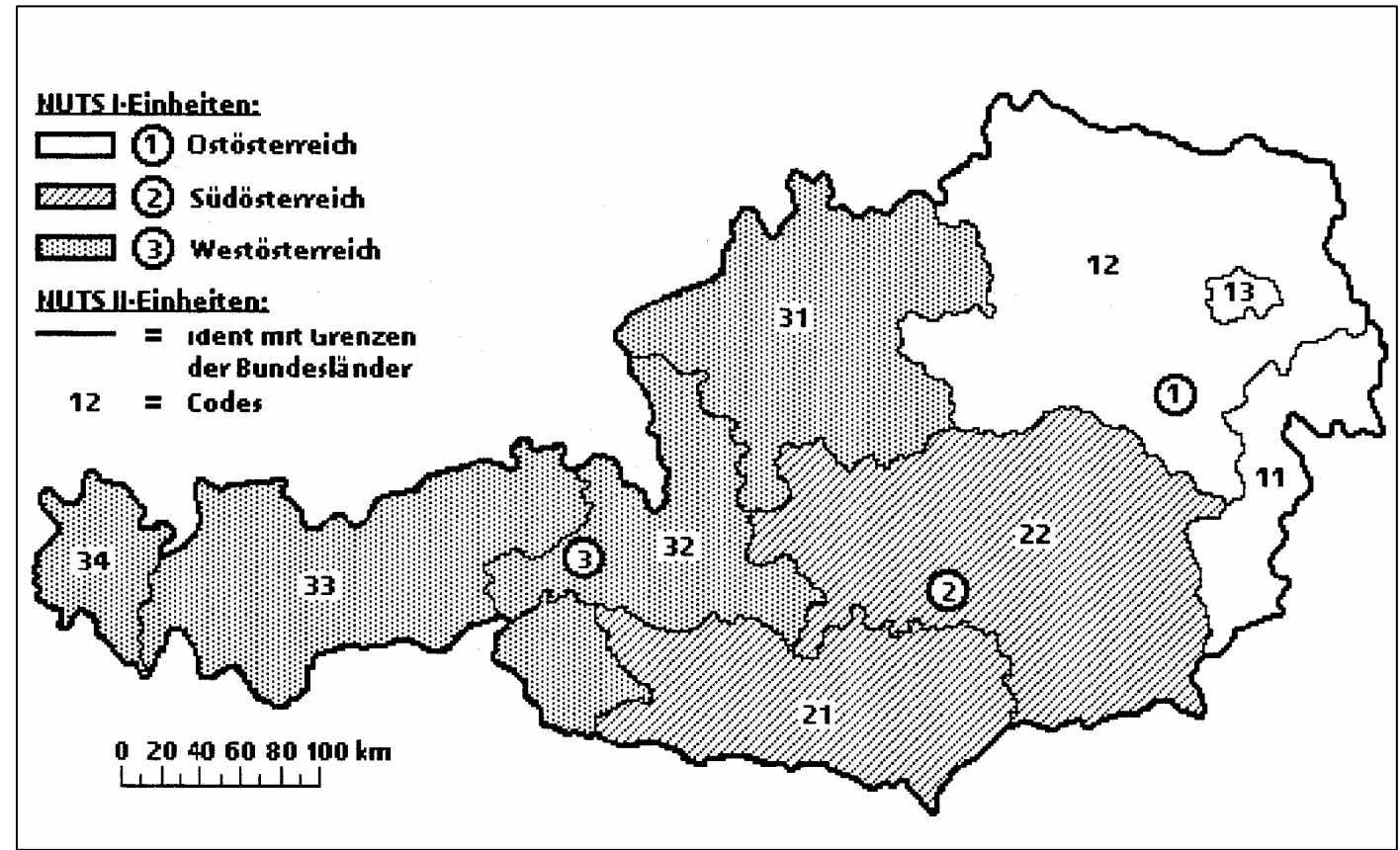

Gliederung in NUTS III-Einheiten

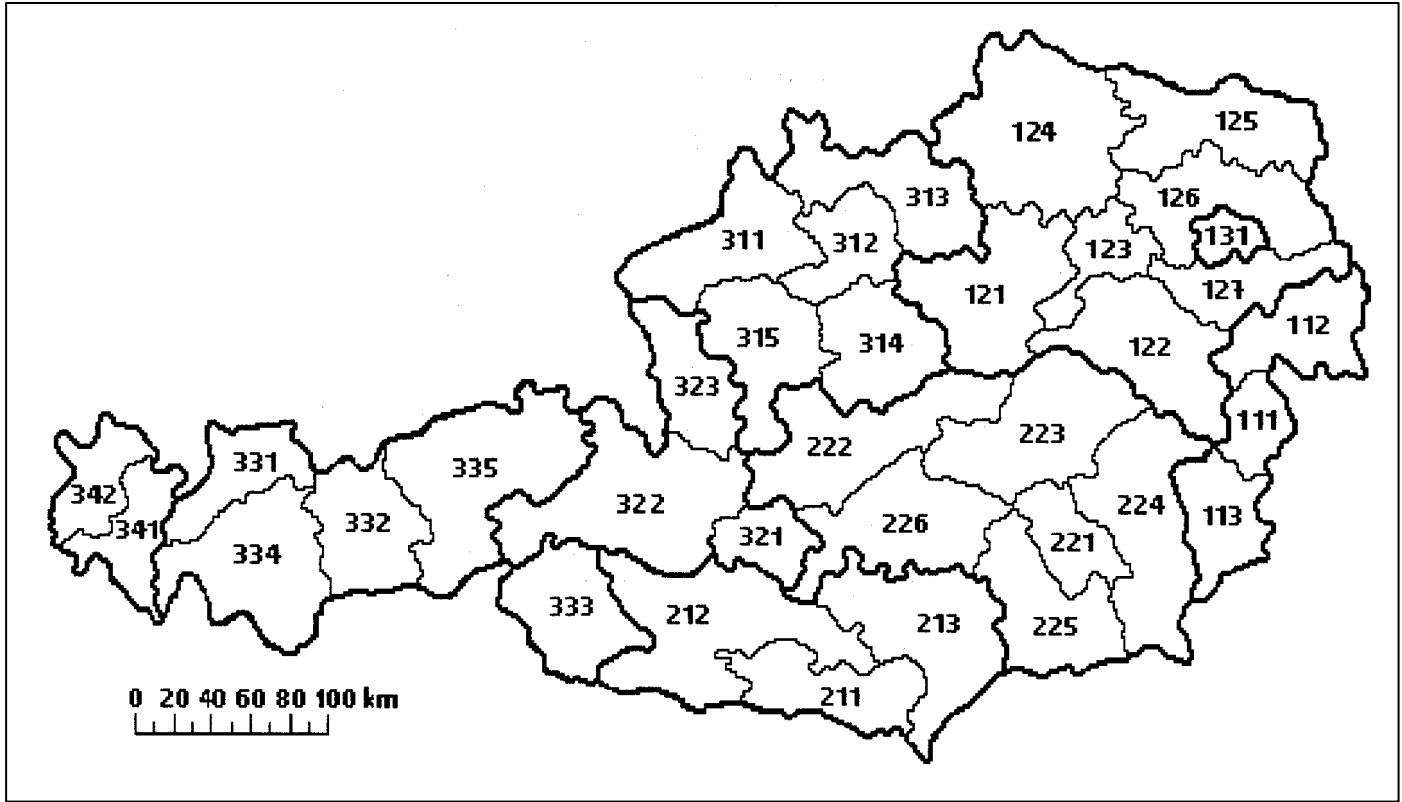

Figure 1: Geographical representation of the nuts-classification Source: Main Association of Austrian Social Security Institutions 
Figure 2: Employees of 1972 and fraction in REV data

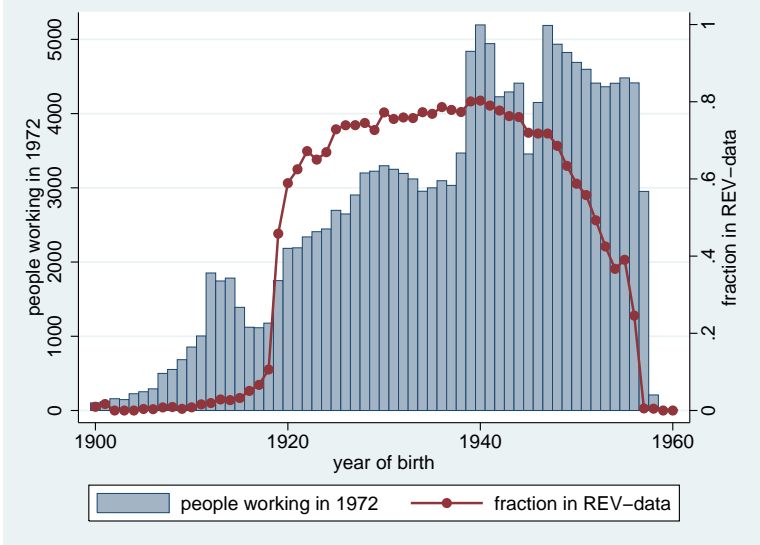

(a) men

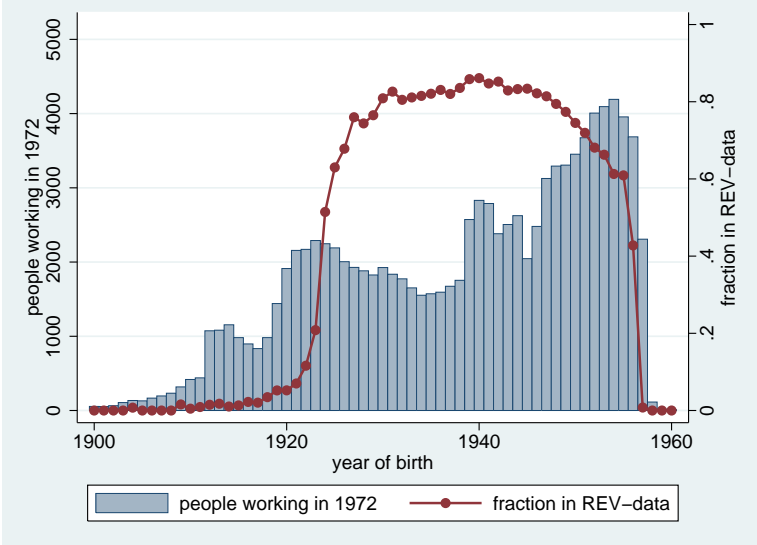

(b) women

Figure 3: Employees of 1972 working in 1985 (or afterwards) and fraction in REV data

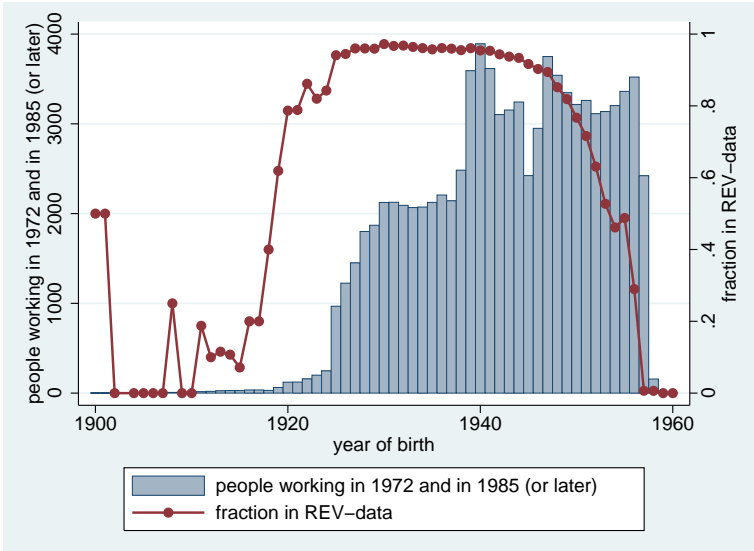

(a) men

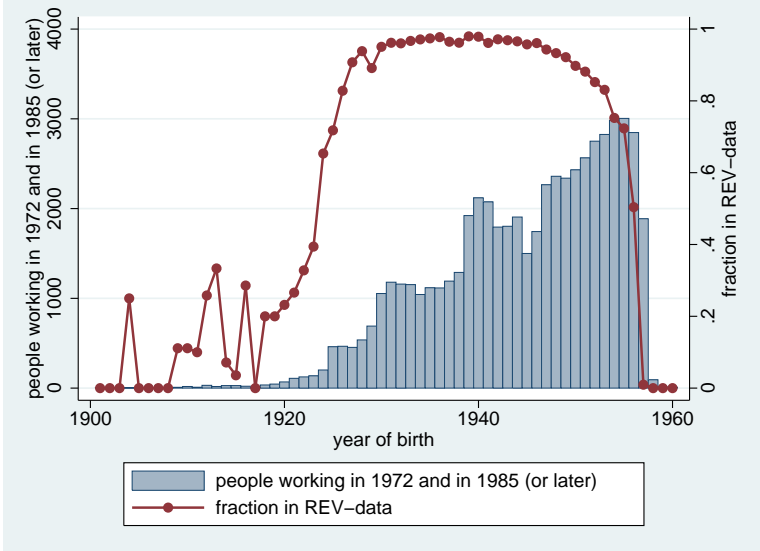

(b) women

Notes: Both figures represent $10 \%$ random samples of the respective subgroup. 


\section{Tables}

Table 1: Detailed description of the different types of qualifications

\begin{tabular}{ll}
\hline \hline qual & type \\
\hline 01 & Bestätigte Nullzeit \\
& \\
02 & $\begin{array}{l}\text { Ersatzzeit - Ohne Verschulden } \\
\text { des Versicherten zu Unrecht be- } \\
\text { zogene Leistung aus der Arbeit- } \\
\text { slosenversicherung }\end{array}$
\end{tabular}

PV-Pflichtversicherung nach dem ASVG - Angestellter mit wesentlicher bergmännischer Tätigkeit schaftsvollpension Knappschaftsalterspension

PV-Pflichtversicherung nach dem ASVG (knappschaftl. PV, Arb. mit wesentlich bergmännische Tätigkeit) PV-Pflichtversicherung nach dem ASVG (knappschaftl. PV, Arb. + Gewinnhauertätigkeit) Ersatzzeit für die Zeit in der Mangels Notlage kein Anspruch auf Notstandshilfe besteht (§ 34/1 AIVG)

PV-Pflichtversicherung nach dem ASVG - Angestellter

description

"Hat ein Pensionsversicherungsträger im Zuge eines Datenergänzungsverfahrens festgestellt, daß für einen Zeitraum, den der Versicherte als Versicherungszeit angab, keine für die österreichische Sozialversicherung bedeutsamen Zeiten vorliegen, meldet er diesen Zeitraum mit Qualifikation 01 an den Hauptverband. Diese Meldung obliegt im Regelfall dem leistungszuständigen Pensionsversicherungsträger."

"Alle Zeiten, für die eine Person ohne eigenes Verschulden Geldleistungen aus der Arbeitslosenversicherung bezogen hat, wenn sie wegen Zeitablauf nicht mehr rückgefordert werden können. Zeiten in denen aus eigenem Verschulden eine Geldleistung aus der Arbeitslosenversicherung nicht mehr rückforderbar ausbezahlt worden war, werden von der Arbeitsmarktverwaltung aus der Versicherungsdatei gelöscht!"

Bezug eines Sonderruhegeldes "Alle Zeiten, in denen der Bezug eines Sonderruhegeldes gemäß Artikel X nach dem NSchG des Nachtschicht- Schwerarbeitsgesetzes vorliegt. Sonderruhegeld aufgrund von Knappschaftszeiten sind ab 1.1.2005 vom Versicherungsträger 05 unter der Qualifikation Y5 zu melden."

Bezug von Knappschaftssold Alle Zeiten, in denen ein bescheidmäßig zuerkannter Anspruch auf Knappschaftssold besteht.

Bezug einer Invaliditäts- / "Alle Zeiten, in denen ein bescheidmäßig zuerkannter Anspruch auf eine Leistung Berufsunfähigkeits- / Knapp- aus dem Versicherungsfall der geminderten Arbeitsfähigkeit nach dem ASVG, mit Ausnahme von Knappschaftspension, besteht. Der Tatbestand der Knappschaftsvollpension ist ab 1.1.2005 vom Versicherungsträger 05 unter der Qualifikation Y7 zu melden."

Bezug einer Erwerb- "Alle Zeiten, in denen ein bescheidmäßig zuerkannter Anspruch auf eine Leistung sunfähigkeits- / Knapp- aus dem Versicherungsfall der dauernden Erwerbsunfähigkeit nach dem GSVG, schaftspension dem BSVG (LZVG) oder auf Knappschaftspension nach dem ASVG wegen Dienstunfähigkeit besteht."

Bezug einer Alterspension / "Alle Zeiten, in denen ein bescheidmäßig zuerkannter Anspruch auf eine Leistung aus dem Versicherungsfall des Alters nach dem ASVG (auch Knappschaftsalterspension) oder auf eine Alterspension nach dem GSVG oder BSVG besteht. Der Tatbestand der Knappschaftsalterspension ist ab 1.1.2005 vom Versicherungsträger 05 unter der Qualifikation Y9 zu melden."

PV-Pflichtversicherung nach "Alle Pflichtversicherungszeiten in der Pensionsversicherung der Arbeiter nach dem ASVG - Arbeiter dem ASVG einschließlich der Pflichtversicherungszeiten als Arbeiter in der knappschaftlichen Pensionsversicherung mit Ausnahme der Sonderfälle mit Qualifikation 11 - 12, B1, B2, C1, D1 sowie G1 - G6. Pflichtversicherungszeiten im knappschaftlichen PV-Zweig sind ab 1.1.2005 vom Versicherungsträger 05 unter der Qualifikation Y1 zu melden."

Continued on next page...
"Alle Pflichtversicherungszeiten als Arbeiter in der knappschaftlichen Pensionsversicherung für Personen mit wesentlich bergmännischer Tätigkeit (lt. Anlage 9 zum ASVG)."

"Alle Pflichtversicherungszeiten als Arbeiter in der knappschaftlichen Pensionsversicherung für Personen mit Gewinnhauertätigkeit (lt. Anlage 10 zum ASVG)."

"Alle Zeiten gemäß des $\S 34$ Abs. 1 AIVG, die gemäß den Bestimmungen des $\S$ 227 Abs. 1 Z 5 ASVG als Ersatzzeiten in der Pensionsversicherung angerechnet werden."

"Alle Pflichtversicherungszeiten in der Pensionsversicherung der Angestellten einschließlich der Pflichtversicherungszeiten als Angestellter in der knappschaftlichen Pensionsversicherung, mit Ausnahme der Sonderfälle mit Qualifikation 15, 16, B4 und C4. Pflichtversicherungszeiten im knappschaftlichen PV-Zweig sind ab 1.1.2005 vom Versicherungsträger 05 unter der Qualifikation Y4 zu melden.”

"Alle Pflichtversicherungszeiten als Angestellter in der knappschaftlichen Pensionsversicherung für Personen mit wesentlich bergmännischer Tätigkeit (lt. Anlage 9 zum ASVG)." 
... table 1 continued

\begin{tabular}{|c|c|c|}
\hline qual & type & description \\
\hline 16 & $\begin{array}{l}\text { PV-Pflichtversicherung nach } \\
\text { dem ASVG - Angestellter mit } \\
\text { Gewinnhauertätigkeit }\end{array}$ & $\begin{array}{l}\text { "Alle Pflichtversicherungszeiten als Angestellter in der knappschaftlichen Pen- } \\
\text { sionsversicherung für Personen mit Gewinnhauertätigkeit (lt. Anlage } 10 \text { zum } \\
\text { ASVG)." }\end{array}$ \\
\hline 17 & $\begin{array}{l}\text { Zeit einer Beitragsvorschrei- } \\
\text { bung zur Pflichtversicherung } \\
\text { nach dem GSVG / FSVG / } \\
\text { BSVG }\end{array}$ & $\begin{array}{l}\text { "Alle Zeiten, in denen eine Pflichtversicherung nach dem GSVG (FSVG) oder } \\
\text { BSVG besteht, die vorgeschriebenen Beiträge aber (noch) nicht bezahlt wurden. } \\
\text { Zeiträume unter der Qualifikation } 17 \text { beziehen sich auf gespeicherte Pflichtver- } \\
\text { sicherungszeiten mit Qualifikation } 18,19, \text { F1 oder F2 und werden parallel zu } \\
\text { diesen gespeichert." }\end{array}$ \\
\hline 18 & $\begin{array}{l}\text { Pflichtversicherung nach dem } \\
\text { GSVG }\end{array}$ & $\begin{array}{l}\text { "Alle Pflichtversicherungszeiten nach dem GSVG (siehe Kapitel D.6.17.) mit Aus- } \\
\text { nahme der Zeiten einer selbständigen Erwerbstätigkeit gemäß } \S 2 \text { Abs. } 1 \text { Z } 4 \\
\text { GSVG (siehe Kapitel D.6.F3.)." }\end{array}$ \\
\hline 19 & $\begin{array}{l}\text { Pflichtversicherung nach dem } \\
\text { BSVG (Betriebsführer) }\end{array}$ & $\begin{array}{l}\text { "Alle Pflichtversicherungszeiten nach dem BSVG für in der Land- und } \\
\text { Forstwirtschaft selbständig erwerbstätige Personen, die auf ihre Rechnung und } \\
\text { Gefahr einen land- und forstwirtschaftlichen Betrieb führen oder auf deren Rech- } \\
\text { nung und Gefahr ein solcher Betrieb geführt wird, wenn sie nicht von der } \\
\text { Pflichtversicherung ausgenommen sind (siehe Kapitel D.6.17.)." }\end{array}$ \\
\hline
\end{tabular}

Pflichtversicherung nach dem BSVG (Angehöriger)

"Alle Pflichtversicherungszeiten nach dem BSVG für die Angehörigen von Personen, die auf eigene Rechnung und Gefahr einen land- und forstwirtschaftlichen Betrieb führen oder auf deren Rechnung und Gefahr ein solcher Betrieb geführt wird, wenn sie nicht von der Pflichtversicherung ausgenommen sind (siehe Kapitel D.6.17.)."

21 Überweisungsbetrag an den Dienstgeber stgeber für Versicherungszeiten im PV-Zweig der 'Arbeiter' nach dem ASVG (Arb. + knappschaftl. PV)

Freiwillige Weiterversicherung nach dem ASVG (Ang. + knappschaftl. PV) nach dem GSVG oder FSVG triebsführer) Versicherten in ein pensionsversicherungsfreies Dienstverhältnis an den Dienstgeber einen Überweisungsbetrag gemäß § 308 ASVG, § 172 GSVG, § 164 BSVG oder $\S 64$ NVG leistet oder die Beiträge rückerstattet. Gilt auch für Sonderverträge mit internationalen Vereinigungen (UNIDO, IAEO und ähnl.)."

"Alle Zeiten eines pensionsversicherungsfreien Dienstverhältnisses, für die ein Pensionsversicherungsträger nach dem Ausscheiden eines Versicherten aus diesem vom Dienstgeber einen Überweisungsbetrag gemäß $\S \S 311$ und 314 ASVG erhält. Zeiten im Rahmen der knappschaftlichen Pensionsversicherung sind ab 1.1.2005 vom Versicherungsträger 05 unter der Qualifikation Y2 zu melden. Im knappschaftlichen Bereich ist eine Unterscheidung zwischen Arbeiter und Angestellten nicht erforderlich, da bei der Verdichtung ein diesbezüglicher Umstand nicht berücksichtigt wird."

"Zeiten einer Anhaltung, in Ansehung derer ein österreichisches Gericht rechtskräftig einen Entschädigungsanspruch für strafgerichtliche Anhaltung oder Verurteilung zuerkannt hat und für die der Bund an den zuständigen Pensionsversicherungsträger Beiträge entrichtet (§ 506a ASVG, § 117 GSVG, § 108 BSVG). Zeiten im Rahmen der knappschaftlichen Pensionsversicherung sind ab 1.1.2005 vom Versicherungsträger 05 unter der Qualifikation Y3 zu melden."

"Zeiten, für die Beiträge außerhalb der gesetzlich vorgesehenen Fristen entrichtet wurden, und die daher für die Anwartschaftsfeststellung in der Pensionsversicherung unwirksam sind. Zeiten, für die aufgrund der eingetretenen Verjährung keine Beiträge mehr eingefordert werden können oder für die vom Versicherungsträger die Beiträge als uneinbringlich abgeschrieben wurden, sind in die Versicherungsdatei nicht zu melden."

"Alle Zeiten, für die Beiträge zur freiwilligen Weiterversicherung in der Pensionsversicherung der Arbeiter, auch als Arbeiter in der knappschaftlichen Pensionsversicherung entrichtet werden. Die Zeiten in der knappschaftlichen Pensionsversicherung sind ab 1.1.2005 vom Versicherungsträger 05 unter der Qualifikation Z5 zu melden."

"Alle Zeiten, für die in der Pensionsversicherung der Angestellten, auch in der knappschaftlichen Pensionsversicherung als Angestellte Beiträge zur freiwilligen Weiterversicherung entrichtet werden. Die Zeiten in der knappschaftlichen Pensionsversicherung sind ab 1.1.2005 vom Versicherungsträger 05 unter der Qualifikation Z6 zu melden."

"Alle Zeiten, für die Beiträge zur freiwilligen Weiterversicherung nach dem GSVG oder FSVG entrichtet werden, sowie Zeiten der Selbstversicherung von Verpächtern von Betrieben gemäß $§ 234$ GSVG."

"Alle Zeiten, für die unter Kapitel D.6.19. Punkt a) beschriebenen Personen nach Beendigung der Pflichtversicherung Beiträge zur freiwilligen Weiterversicherung nach dem BSVG entrichtet werden (siehe Kapitel D.6.19.)." 
... table 1 continued

\begin{tabular}{lll}
\hline \hline qual & type & description \\
\hline 29 & Freiwillige Weiterversicherung & "Alle Zeiten, für die unter Kapitel D.6.20. Punkt a) beschriebenen Personen nach \\
& nach dem BSVG (Angehöriger) & Beendigung der Pflichtversicherung Beiträge zur freiwilligen Weiterversicherung \\
& & nach dem BSVG entrichten (siehe Kapitel D.6.20.)."
\end{tabular}

2A Verjährter Beitragsgrundlagen"Unter oben genannter Qualifikation sind jene Beitragsgrundlagenteile zu melden, teil (GSVG) für die aufgrund der eingetretenen Verjährung keine Beiträge mehr eingefordert werden können und die daher in der Pensionsversicherung im Rahmen der Verdichtung außer Ansatz zu lassen sind."

$30 \quad$ Mittlere Schule

"Alle Zeiten des Besuches einer inländischen öffentlichen oder mit dem Öffentlichkeitsrecht ausgestatteten mittleren Schule mit mindestens zweijährigem Bildungsgang, wobei erstmalig jenes Schuljahr gemeldet wird, das in dem Kalenderjahr beginnt, in dem der Schüler das 15. Lebensjahr vollendet. Ausgenommen von dieser Meldung sind berufsbegleitende Schulen (Berufs-, Fachschulen etc.), da die betreffenden Personen als Lehrling ohnehin gemeldet sind und auf diesem Weg Versicherungszeiten erwerben und Zeiten eines Schulbesuches, für die nachträglich Beiträge bezahlt wurden (siehe Kapitel D.6.H1)."

31 Höhere Schule "Alle Zeiten des Besuches einer inländischen höheren Schule, Akademie oder verwandten Lehranstalt in dem für diese Schularten vorgeschriebenen normalen Ausbildungsgang (wenn nachträglich keine Beiträge entrichtet wurden), wobei erstmalig jenes Schuljahr gemeldet wird, das in dem Kalenderjahr beginnt, in dem der Schüler das 15. Lebensjahr vollendet (siehe Kapitel D.6.H2.)."

Hochschule

"Alle Zeiten des Besuches einer inländischen Hochschule bzw. Kunstakademie oder Kunsthochschule in dem für diese Schul-(Studien)art vorgeschriebenen normalen Ausbildungs-(Studien)gang, wenn nachträglich keine Beiträge entrichtet wurden (siehe Kapitel D.6.H3.)."

"Alle Zeiten des ordentlichen oder außerordentlichen Präsenzdienstes entsprechend den Bestimmungen des österreichischen Wehrgesetzes. Zeiten von Inspektionen, Instruktionen (bis 31.12.1976) sowie Truppen- und Kaderübungen gemäß § 16 des Wehrgesetzes sind ebenfalls dieser Qualifikation zuzuordnen. Die Speicherung dieser Qualifikation erfolgt nur durch den zuständigen Pensionsversicherungsträger."

Wochengeldbezug (auf Dienst-

"Alle Zeiten, in denen weibliche Personen auf Grund eines ASVGDienstverhältnisses gemäß $\S 162$ ASVG Wochengeld erhalten, Wochengeldbezug in der Schutzfrist gemäß $\S 122$ Abs. 2 Z 2 lit. a und b und Abs. 3 ASVG, Wochengeldbezug gem. § 84 B-KUVG ab 1.1.1999, oder Zeiten des Ruhens des Wochengeldes $\S 166$ Abs. 1 Z 1 ASVG. Teilweises oder gänzliches Ruhen des Wochengeldes gemäß $\S 166$ Abs. 1 Z 2 bzw. Z 3 ASVG bewirkt ein Fortbestehen der Pflichtversicherung (Teilentgelt). Ausgenommen sind Bezieher von Wochengeld mit Qualifikation 35."

Wochengeldbezug (Sonderfälle) "Alle Zeiten, in denen weibliche Personen, die als Teilversicherte gemäß § 7 Z 1 lit. a - f (z. B. Rechtsanwaltsanwärter) oder $\S 8$ Abs. $1 \mathrm{Z} 4$ (freiberuflich tätige bildende Künstler, Pflichtmitglieder der Tierärztekammern und Pflichtmitglieder der Österreichischen Dentistenkammer) gelten, oder aufgrund des Bezuges von Arbeitslosengeld, Karenzurlaubsgeld, Notstandshilfe oder einer Sonderunterstützung gemäß $\S 29$ MSCHG oder aufgrund einer durch eine Beschäftigung bei einer internationalen Organisation (IAEO, UNIDO) oder verschiedenen Gemeinde- und Landesbediensteten (eigene Ruhensgenussbestimmungen) begründeten Krankenversicherung nach dem ASVG Wochengeld beziehen, Zeiten des Ruhens dieses


bezug während dieser die Versicherte Urlaubsentschädigung, Urlaubsabfindung oder Kündigungsentschädigung bezieht. In jenen Fällen, in denen sich die Zuständigkeit des Krankenversicherungsträgers ändert, weil während des Ruhens eines Arbeitslosengeldbezuges wegen Bezug einer Urlaubsabfindung oder Urlaubsentschädigung der Wohnort des Versicherten nicht im gleichen Bundesland wie der Beschäftigungsort liegt, ist ein Wochengeldbezug ebenfalls unter der Qualifikation 35 zu melden."

"Krankengeldbezug in der Schutzfrist gemäß $\S 122$ Abs. 2 Z 2 lit. a und b geberkontonummer bezogen) ASVG und Zeiten, während derer der Anspruch auf Krankengeld ausschließlich gemäß $\S 143$ Abs. 1 Z 2 ASVG ruhte oder gemäß $\S 143$ Abs. 6 ASVG teilweise ruhte. Ebenso alle Zeiten, für die ein Anspruch auf Krankengeld vom Versicherungsträger festgestellt, das Krankengeld vom Anspruchsberechtigten aber nicht behoben wurde. Andere Ruhensgründe (z.B. Übergangsgeldbezug) gelten als Unterbrechung des Krankengeldbezuges - Qualifikation 00. Weiters alle Zeiten gem. § 84 B-KUVG analog den oben angeführten Bestimmungen des ASVG (ab 1.1.1999). Ausgenommen sind Bezieher von Krankengeld mit Qualifikation 37." 


\begin{tabular}{|c|c|c|c|}
\hline qual & type & & description \\
\hline 37 & $\begin{array}{l}\text { Krankengeldbezug } \\
\text { derfälle) }\end{array}$ & (Son- & $\begin{array}{l}\text { "Alle Zeiten, in denen Personen, die als Teilversicherte gemäß } \S 7 \mathrm{Z} 1 \text { lit. a } \\
\text { - f (z.B. Rechtsanwaltsanwärter) oder } \S 8 \mathrm{Abs} .1 \mathrm{Z} 4 \text { (freiberuflich tätige } \\
\text { bildende Künstler, Pflichtmitglieder der Tierärztekammern und Pflichtmitglieder } \\
\text { der Österreichischen Dentistenkammer) gelten oder auf Grund des Bezuges von } \\
\text { Arbeitslosengeld, Karenzurlaubsgeld oder Notstandshilfe oder auf Grund einer } \\
\text { durch eine Beschäftigung bei einer internationalen Organisation (IAEO, UNIDO) } \\
\text { oder verschiedenen Gemeinde- und Landesbediensteten (eigene Ruhegenussbes- } \\
\text { timmungen) begründeten Krankenversicherung nach dem ASVG Krankengeld } \\
\text { beziehen oder Zeiten, während derer der Anspruch auf ein solches Krankengeld } \\
\text { gemäß } § 143 \text { Abs. } 1 \mathrm{Z} 2 \text { ASVG ruhte oder gemäß } \S 143 \text { Abs. } 6 \text { ASVG teil- } \\
\text { weise ruhte. Ebenso alle Zeiten, für die ein Anspruch auf Krankengeld vom Ver- } \\
\text { sicherungsträger festgestellt, das Krankengeld vom Anspruchsberechtigten aber } \\
\text { nicht behoben wurde. Andere Ruhensgründe gelten als Unterbrechung dieses } \\
\text { Krankengeldbezuges (z.B. Übergangsgeldbezug). In jenen Fällen, in denen sich die } \\
\text { Zuständigkeit des Krankenversicherungsträgers ändert, weil während des Ruhens } \\
\text { eines Arbeitslosengeldbezuges wegen Bezug einer Urlaubsabfindung oder Urlaub- } \\
\text { sentschädigung der Wohnort des Versicherten nicht im gleichen Bundesland wie } \\
\text { der Beschäftigungsort liegt, ist ein Krankengeldbezug ebenfalls unter der Quali- } \\
\text { fikation } 37 \text { zu melden." }\end{array}$ \\
\hline
\end{tabular}
oder Notstandshilfe oder Überbrückungshilfe (BGBl.Nr. 174/1963) vorliegt (bis 31.12.1997). Ab 1.1.1998 sind unter dieser Qualifikation nur Zeiten eines Arbeitslosengeldbezuges zu speichern (siehe Kapitel D.6.C5.) Des Weiteren sind ab 1.1.2004 alle Zeiten, in denen eine Beihilfe zur Deckung des Lebensunterhaltes gem. $\S 20$ Abs. 2 lit.c des AMFG ausschließlich oder gemeinsam mit Arbeitslosengeld bezogen wird, unter dieser Qualifikation zu melden." "Alle Zeiten von Personen, die als arbeitslos im Sinne des AlVG gemeldet sind, jedoch vom Bezug einer Geldleistung aus einem anderen Grund als wegen Arbeitsunwilligkeit, Auflösung des Dienstverhältnisses durch eigenes Verschulden freiwillige Lösung des Dienstverhältnisses ohne triftigen Grund oder Unterlassung der Kontrollmeldung ausgeschlossen sind. Es können aber auch Zeiten einer vorgemerkten Arbeitssuche (ohne Arbeitslosigkeit) unter Qualifikation 39 gemeldet werden."

3A Präsenzdienst- bzw. Ausbildungsdienst beim österr. Bundesheer

"Diese Qualifikation dient dem Krankenversicherungsträger zum Melden von Präsenzdienst bzw. Ausbildungszeiten im laufenden Verfahren und wird automatisch aufgrund einer Meldung des Heeresdatenverarbeitungsamtes (HDVA) vom Hauptverband im Namen des jeweilig zuständigen Krankenversicherungsanspruches für den Versicherten und allfällig vorliegender Angehöriger." "Unter obgenannter Qualifikation sind jene Zeiten zu melden, für die eine Person nach $\S 61$ Abs. 9 bzw. Abs. 10 nach dem Wehrgesetz 2001 im Rahmen als Zeitsoldat Wehrdienst geleistet hat. Jene Zeit, die bereits heute nach $\S 8$ Abs. 1 Z 5 ASVG als Pflichtversicherungszeit eingespeichert wird, bleibt hievon unberührt." "Unter obgenannter Qualifikation ist die Bemessungsgrundlage nach § 44 Abs. $1 \mathrm{Z} 12$ ASVG für jene Zeiten des Wochengeldbezuges zu melden, die mittels Qualifikation 34 bzw. 35 in die Versicherungsdatei des HVB eingespeichert wurden. Darüber hinaus ist auch jener Wochengeldbezug zu berücksichtigen, der nicht als Zeitenmeldung (34 bzw. 35) vorliegt, sondern lediglich in Form von Teilentgelttagen im Rahmen der Beitragsgrundlagenmeldungen beim entsprechenden Beschäftigungsverhältnis angeführt wird (Lehrlinge)." Z 14 ASVG für jene Zeiten des Krankengeldbezuges zu melden, die mittels Qualifikation 36 bzw. 37 in die Versicherungsdatei des HVB eingespeichert wurden. Darüber hinaus ist auch jener Krankengeld zu berücksichtigen, dass nur $50 \%$ gebührt und aus den dadurch keine Qualifikationszeitenmeldungen (36 oder 37) resultieren. Generell sind jegliche Zeiten des Krankengeldbezuges, die im Rahmen der Beitragsgrundlagenmeldungen nur als Teilentgelttage gemeldet werden, zu berücksichtigen. Beim Krankengeldbezug für Versicherte nach dem ALVG gelten die jeweiligen Beitragsgrundlagen für das ALVG (§ 44 Abs. 1 Z 13)." 


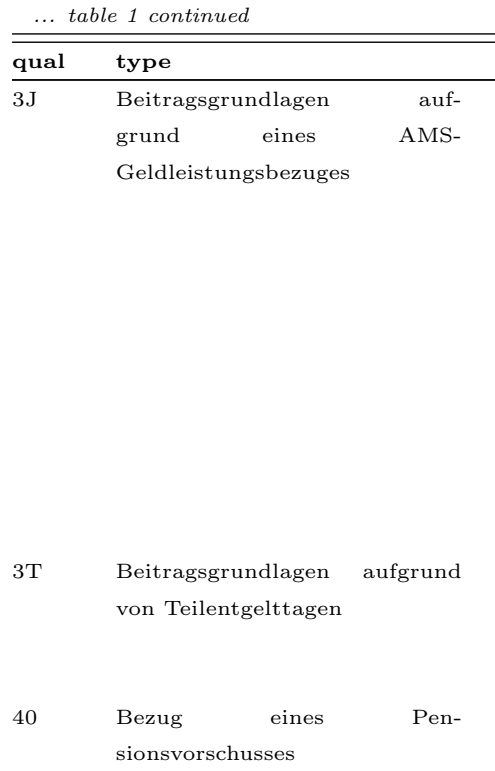

description

"U

Unter obgenannter Qualifikation sind die Beitragsgrundlagen für jene Zeiten zu recht bezogene Leistung aus der ALV) 13 - Ersatzzeit für die Zeit, in der mangels Notlage kein Anspruch auf Notstandshilfe besteht (§ 34/1 AIVG) 38 - (Bezug von Arbeitslosengeld) 48 - (Bezug einer Sonderunterstützung) 56 - (Ersatzzeit aufgrund des Ruhens eines ALG-Bezuges wegen Urlaubsabfindung bzw. Urlaubsentschädigung) 81 - (Sondernotstandhilfe) A1 - (Bezug einer Beihilfe gem. $§ 20$ Abs. 2 lit.c AMFG - Arbeiter) A4 - (Bezug einer Beihilfe gem. § 20 Abs. 2 lit.c AMFG - Angestellter) BK - (Bezug von Kombilohn - Bezugszeiten) C5 - (Bezug von Notstands- oder Überbrückungshilfe) N4 - (Weiterbildungsgeld nach ALVG 1977 ab dem 45 Lebensjahr) NU - (Weiterbildungsgeld nach ALVG 1977 vor dem 45.Lebensjahr) QA - Vormerkung zur Kranken- und Pensionsversicherung nach dem ASVG in die Versicherungsdatei des HVB eingespeichert wurden."

"Unter oben genannter Qualifikation sind die Beitragsgrundlagen aufgrund von Teilentgelttagen, die bei den einzelnen Beschäftigungsverhältnissen (z.B. Qual 10 oder 14 Meldungen) lediglich als Tagesanzahl gemeldet werden, jedoch bei der dazugehörigen Beitragsgrundlage nicht beinhaltet ist, zu melden."

sionsvorschusse

"Alle Zeiten einer Bevorschussung von Pensionsleistungen gemäß $\S 23$ AlVG. Bei ablehnendem Pensionsbescheid oder einer späteren Pensionszuerkennung als ursprünglich bekannt gegeben ändert die Arbeitsmarktverwaltung die Qualifikation für den betroffenen Zeitraum auf 38 (Arbeitslosengeldbezug)."

"Alle Zeiten eines Bezuges von Karenzurlaubsgeld gemäß $\S 26$ (ausgenommen Abs. $1 \mathrm{Z} 3$ ) und Karenzgeldbezug $\S 26 \mathrm{a}$ (ausgenommen Abs. $1 \mathrm{Z} 3$ ) AlVG bzw. $\S 2$ Abs. 1 KGG." "Alle Zeiten, in denen ein bescheidmäßig zuerkannter Anspruch auf eine grund von mindestens $50 \%$ Erwerbsfähigkeitseinbuße Versehrtenrente aus der gesetzlichen Unfallversicherung aufgrund einer Erwerbsfähigkeitseinbuße von mindestens $50 \%$ (Schwerversehrte) besteht."

"Alle Zeiten eines Bezuges von Karenzurlaubsgeld gemäß $\S 26$ Abs. 1 Z 3 und $\S 26 \mathrm{a}$ Abs. $1 \mathrm{Z} 3$ AlVG bzw. § 2 Abs. 1 KGG (bei Annahme eines Kindes an Kindes statt)."

"Alle Zeiten einer unentgeltlichen beruflichen Ausbildung eines Beschädigten im dem KOVG

Beschädigtenrente nach dem KOVG Sinne des KOVG."

"Alle Zeiten einer Untersuchungshaft, wenn das strafgerichtliche Verfahren gemäß $\S 90$ oder $\S 109$ der Strafprozessordnung eingestellt wurde oder mit einem Freispruch endete."

"Alle Zeiten einer Strafhaft auf Grund einer Tat, die nach den österreichischen Gesetzen im Zeitpunkt der Begehung der Tat strafbar war, nach den österreichischen Gesetzen bei Eintritt des Versicherungsfalles jedoch nicht mehr strafbar ist."

"Alle Zeiten, in denen der Bezug einer Beschädigtenrente nach dem KOVG 1957, dem HVG oder dem OFG auf Grund einer Erwerbsfähigkeitseinbuße von mindestens $70 \%$ vorliegt."

"Alle Zeiten, in denen der Bezug einer Sonderunterstützung nach einem Sonderunterstützungsgesetz (BG 117/67 oder BG 642/73) vorliegt."

"Diese Qualifikation ist für jene in der Pensionsversicherung nach dem ASVG, GSVG und BSVG pflichtversicherten bzw. weiterversicherten weiblichen Personen einzugeben, die ab dem 1.1.1972 von einem lebendgeborenen Kind entbunden wurden ( $§ 227 \mathrm{Z} 4 \mathrm{ASVG}$ ). Bei Meldung durch die Pensionsversicherungsträger im Datenergänzungsverfahren können auch Lebendgeburten, die im Jahr 1971 liegen, gemeldet werden, weil die anrechenbare Ersatzzeit von einem Kalenderjahr in den Zeitraum der allgemeinen elektronischen Datenspeicherung ab 00.01.1972 reicht. Für solche Meldungen ist jedenfalls ein gesonderter Eingabesatz zu verwenden. Weiters können Familienangehörige sowie Dienstnehmer, die in einem pensionsversicherungsfreien Dienstverhältnis stehen, gemeldet werden. Die Meldung ist für jedes lebendgeborene Kind wie ein 1-tägiges Versicherungsverhältnis zum Geburtstag des Kindes zu veranlassen. Bei Mehrlingsgeburten an einem Kalendertag ist nur eine Meldung zu beauftragen."

4B KV-Pfl.-Vers. $\S 2 / 1 / 1-\S 2 / 1 / 3$ GSVG (Geldleistung)

"Alle Zeiten der KV-Pflichtversicherung nach $\S 2$ Abs. $1 / 1$ bis Abs. $1 / 3$ GSVG (Kammermitglieder, Gesellschafter, Geschäftsführer) - SACHLEISTUNG."

"Alle Zeiten der KV-Pflichtversicherung nach $\S 2$ Abs. $1 / 1$ bis Abs. 1/3 GSVG (Kammermitglieder, Gesellschafter, Geschäftsführer) - GELDLEISTUNGSANSPRUCH."

Continued on next page... 


\begin{tabular}{|c|c|c|}
\hline qual & type & description \\
\hline $4 \mathrm{C}$ & $\begin{array}{l}\text { KV-Pfl.-Vers. } § 2 / 1 / 4 \text { GSVG } \\
\text { (Sachleistung) }\end{array}$ & $\begin{array}{l}\text { "Alle Zeiten der KV-Pflichtversicherung nach } \S 2 \text { Abs. 1/4 GSVG (klassische } \\
\text { selbständige Erwerbstätige) - SACHLEISTUNGSANSPRUCH." }\end{array}$ \\
\hline $4 \mathrm{D}$ & $\begin{array}{l}\text { KV-Pfl.-Vers. } § 2 / 1 / 4 \text { GSVG } \\
\text { (Geldleistung) }\end{array}$ & $\begin{array}{l}\text { "Alle Zeiten der KV-Pflichtversicherung nach } \S 2 \text { Abs. } 1 / 4 \text { GSVG (klassische } \\
\text { selbständige Erwerbstätige) - GELDLEISTUNGSANSPRUCH." }\end{array}$ \\
\hline $4 \mathrm{E}$ & $\begin{array}{l}\text { KV-Pfl.-Vers. } \S \S 14 / \mathrm{A}, 14 / \mathrm{B} \\
\text { GSVG (Sachleistung) }\end{array}$ & $\begin{array}{l}\text { "Alle Zeiten der KV-Pflichtversicherung nach } \S 14 \text { a GSVG (Selbstversicherung) } \\
\text { und } \S 14 \mathrm{~b} \text { GSVG (Pflichtversicherung) - SACHLEISTUNGSANSPRUCH." }\end{array}$ \\
\hline $4 \mathrm{~F}$ & $\begin{array}{l}\text { KV-Pfl.-Vers. } \S \S 14 / \mathrm{A}, 14 / \mathrm{B} \\
\text { GSVG (Geldleistung) }\end{array}$ & $\begin{array}{l}\text { "Alle Zeiten der KV-Pflichtversicherung nach } \S 14 \text { a GSVG (Selbstversicherung) } \\
\text { und } \S 14 \mathrm{~b} \text { GSVG (Pflichtversicherung) - GELDLEISTUNG." }\end{array}$ \\
\hline $4 \mathrm{G}$ & $\begin{array}{l}\text { KV-Weiterversicherung } \\
\text { (GSVG) - Sachleistung }\end{array}$ & $\begin{array}{l}\text { "Alle Zeiten der KV-Pflichtversicherung aufgrund einer freiwilligen Weiterver- } \\
\text { sicherung nach dem GSVG - SACHLEISTUNG." }\end{array}$ \\
\hline $4 \mathrm{H}$ & $\begin{array}{l}\text { KV-Weiterversicherung } \\
\text { (GSVG) - Geldleistung }\end{array}$ & $\begin{array}{l}\text { "Alle Zeiten der KV-Pflichtversicherung aufgrund einer freiwilligen Weiterver- } \\
\text { sicherung nach dem GSVG - GELDLEISTUNG." }\end{array}$ \\
\hline $4 \mathrm{I}$ & $\begin{array}{l}\text { KV-Pensionisten (GSVG) - } \\
\text { Sachleistung }\end{array}$ & $\begin{array}{l}\text { "Alle Zeiten der KV-Pflichtversicherung aufgrund eines Pensionsbezuges nach dem } \\
\text { GSVG - SACHLEISTUNG." }\end{array}$ \\
\hline $4 \mathrm{~J}$ & $\begin{array}{l}\text { KV-Pensionisten (GSVG) } \\
\text { Geldleistung }\end{array}$ & $\begin{array}{l}\text { "Alle Zeiten der KV-Pflichtversicherung aufgrund eines Pensionsbezuges nach dem } \\
\text { GSVG - GELDLEISTUNG." }\end{array}$ \\
\hline $4 \mathrm{~K}$ & $\begin{array}{l}\text { KV-Pflichtversicherung } \\
\text { (GSVG) - Sachleistung }\end{array}$ & $\begin{array}{l}\text { "Alle Zeiten der KV-Pflichtversicherung aufgrund eines vorläufigen Pensions- } \\
\text { bezuges, einer Rehabilitation bzw. eines Bezuges von Übergangsgeld nach dem } \\
\text { GSVG - SACHLEISTUNG" }\end{array}$ \\
\hline $4 \mathrm{~L}$ & $\begin{array}{l}\text { KV-Pflichtversicherung } \\
\text { (GSVG) - Geldleistung }\end{array}$ & $\begin{array}{l}\text { "Alle Zeiten der KV-Pflichtversicherung aufgrund eines vorläufigen Pensions- } \\
\text { bezuges, einer Rehabilitation bzw. eines Bezuges von Übergangsgeld nach dem } \\
\text { GSVG - GELDLEISTUNG" }\end{array}$ \\
\hline $4 \mathrm{M}$ & $\begin{array}{l}\text { KV-Pfl.Vers. } \quad \S 2 / 1 / 4 \text { GSVG } \\
\text { o.Leist.anspr. (Sachleistung) }\end{array}$ & $\begin{array}{l}\text { "Alle Zeiten der KV-Pflichtversicherung nach } \S 2 \text { Abs. 1/4 GSVG ohne Leis- } \\
\text { tungsanspruch (klassische selbständige Erwerbstätige) - SACHLEISTUNG." }\end{array}$ \\
\hline $4 \mathrm{~N}$ & $\begin{array}{l}\text { KV-Pfl.Vers. } \quad \S 2 / 1 / 4 \text { GSVG } \\
\text { o.Leist.anspr. (Geldleistung) }\end{array}$ & $\begin{array}{l}\text { "Alle Zeiten der KV-Pflichtversicherung nach } \S 2 \text { Abs. 1/4 GSVG ohne Leis- } \\
\text { tungsanspruch (klassische selbständige Erwerbstätige) - GELDLEISTUNG." }\end{array}$ \\
\hline $4 \mathrm{P}$ & $\begin{array}{l}\text { KV-Pfl.Vers. } \quad \S \S 14 / \mathrm{A}, 14 / \mathrm{B} \\
\text { GSVG o.Leist.anspr. (Sachleis- } \\
\text { tung) }\end{array}$ & $\begin{array}{l}\text { "Alle Zeiten der KV-Pflichtversicherung nach } \S 14 \text { a (Selbstversicherung) und } \S \\
\text { 14b (Pflichtversicherung) GSVG ohne Leistungsanspruch - SACHLEISTUNG." }\end{array}$ \\
\hline $4 \mathrm{R}$ & $\begin{array}{l}\text { KV-Pfl.Vers. } \S \S 14 / \mathrm{A}, 14 / \mathrm{B} \\
\text { GSVG o.Leist.anspr. (Geldleis- } \\
\text { tung) }\end{array}$ & $\begin{array}{l}\text { "Alle Zeiten der KV-Pflichtversicherung nach } \S 14 \text { a (Selbstversicherung) und } \S \\
\text { 14b (Pflichtversicherung) GSVG ohne Leistungsanspruch - GELDLEISTUNG." }\end{array}$ \\
\hline $4 \mathrm{Z}$ & $\begin{array}{l}\text { Verjährung / Schuldenerlass } \\
\text { (FSVG) }\end{array}$ & $\begin{array}{l}\text { "Alle Zeiten, in denen eine Pflichtversicherung nach dem FSVG besteht, die } \\
\text { Beiträge jedoch nicht mehr eingefordert werden dürfen bzw. verjährt sind." }\end{array}$ \\
\hline 50 & $\begin{array}{l}\text { Nachträglicher Einkauf von } \\
\text { Versicherungszeiten (ASVG / } \\
\text { GSVG) }\end{array}$ & $\begin{array}{l}\text { "Versicherungszeiten, die gemäß Art. VII der } 32 ., \text { Art. VI der } 35 . \text { Novelle } \\
\text { zum ASVG oder gemäß Art. II der } 25 \text {. Novelle zum GSPVG nachträglich durch } \\
\text { Bezahlung der vorgeschriebenen Beiträge erworben wurden." }\end{array}$ \\
\hline 51 & $\begin{array}{l}\text { Ausschluss vom Anspruch auf } \\
\text { Karenzurlaubs- bzw. Karen- } \\
\text { zgeld, weil dieses der Kindes- } \\
\text { vater bezieht bzw. Karenzgeld- } \\
\text { bezug bei Teilzeitbeschäftigung }\end{array}$ & $\begin{array}{l}\text { "Alle Zeiten, in denen die Kindesmutter vom Anspruch auf Karenzurlaubs- } \\
\text { bzw. Karenzgeld ausgeschlossen ist, weil für diesen Zeitraum der Kindesvater } \\
\text { das Karenzurlaubs- bzw. Karenzgeld bezieht (Ausschluss der Kindesmutter } \\
\text { vom Erwerb einer Ersatzzeit gemäß } \S 227 \mathrm{Abs.} 1 \mathrm{Z} 4 \mathrm{ASVG} \text { ). Weiters wird } \\
\text { auch jener Zeitraum dokumentiert, in welchem beide Elternteile Karenzgeld bei } \\
\text { Teilzeitbeschäftigung beziehen." }\end{array}$ \\
\hline 52 & $\begin{array}{l}\text { Überweisungsbetrag vom Dien- } \\
\text { stgeber - Rückzahlung }\end{array}$ & $\begin{array}{l}\text { "Alle Zeiten eines pensionsversicherungsfreien Dienstverhältnisses, für die ein } \\
\text { Pensionsversicherungsträger nach dem Ausscheiden eines Versicherten aus diesem } \\
\text { vom Dienstgeber einen Überweisungsbetrag gemäß } \S 311 \mathrm{ASVG} \text { erhält, wenn für } \\
\text { diese Zeiten vorher ein Überweisungsbetrag gemäß } \S 308 \mathrm{ASVG} \text {, } 172 \mathrm{GSVG} \text {, } \\
164 \text { BSVG oder } \S 64 \mathrm{NVG} \text { geleistet wurde." }\end{array}$ \\
\hline 53 & Zivildienst & $\begin{array}{l}\text { "Alle Zeiten des ordentlichen oder außerordentlichen Zivildienstes aufgrund der } \\
\text { Bestimmungen des Zivildienstgesetzes (BGBl.Nr. 187/1974)." }\end{array}$ \\
\hline 54 & Ausgedinge (BSVG) & $\begin{array}{l}\text { "Neutrale Zeit, die unter Berücksichtigung des gesetzlich vorgesehenen Alters } \\
\text { nach Über(Auf)- gabe eines land(forst)wirtschaftlichen Betriebes erworben wird, } \\
\text { wenn keine Pflichtversicherung in der Pensionsversicherung besteht und aus den } \\
\text { Leistungen der Über(Auf)gabe überwiegend der Lebensunterhalt bestritten wird } \\
\text { (wurde)." }\end{array}$ \\
\hline 55 & $\begin{array}{l}\text { Beschäftigung bei interna- } \\
\text { tionaler Vereinigung und } \\
\text { andere neutrale Zeiten }\end{array}$ & 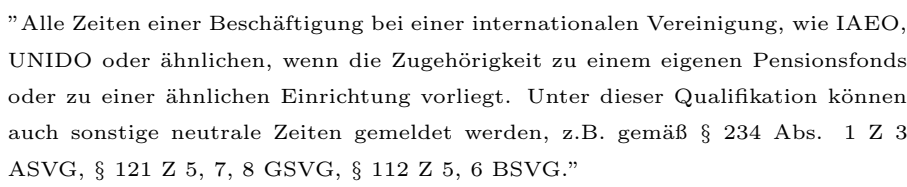 \\
\hline 56 & $\begin{array}{l}\text { Ersatzzeit aufgrund des Ruhens } \\
\text { eines Arbeitslosengeldes } \\
\text { wegen Urlaubsabfindung/- } \\
\text { entschädigung }\end{array}$ & $\begin{array}{l}\text { "Alle Zeiten, in denen ein Arbeitslosengeldbezug ruht, weil der Arbeitslose eine } \\
\text { Urlaubsabfindung oder eine Urlaubsentschädigung erhält ( } § 16 \text { Abs. } 1 \text { lit. } 1 \text { AlVG, } \\
\S 227 \text { Abs. } 1 \text { Z } 5 \text { ASVG)." }\end{array}$ \\
\hline
\end{tabular}


... table 1 continued

\begin{tabular}{lll}
\hline \hline qual & type & description \\
\hline 57 & Besondere Pensionsbeiträge & "Alle Zeiten, für die ein Dienstnehmer aus Anlaß der Aufnahme in ein pensionsver- \\
& sicherungsfreies Dienstverhältnis an den Dienstgeber einen besonderen Pensions- \\
& beitrag geleistet hat, sofern für diese Zeiten ein Überweisungsbetrag gemäß \\
& 311 ASVG geleistet worden ist. Die Zeiten in der knappschaftlichen Pensionsver- \\
& sicherung sind ab 1.1.2005 vom Versicherungsträger 05 unter der Qualifikation Z7 \\
& zu melden." \\
& "Alle Zeiten, in denen Personen in Staaten einer pensionsversicherungspflichti- \\
& gen Beschäftigung nachgehen bzw. behaupten, die mit Österreich ein zwischen- \\
& staatliches Abkommen abgeschlossen haben, das Vorschriften über die An- und \\
& Zusländische & Zusammenrechnung von Versicherungszeiten enthält."
\end{tabular}

$61 \quad$ Beitragszeit gem. $\quad \S 506 \mathrm{~b}$ ASVG

"Alle Zeiten, für die eine Person nach Ausscheiden aus einem im Interesse Österreichs gelegenen Dienstverhältnisses bei einer internationalen Organisation durch Beitragszahlung Pflichtversicherungszeiten für die Dauer dieses Dienstverhältnisses erwirbt." 308 Abs. 4 ASVG

"Alle Zeiten, für die im Zusammenhang mit einer Beurlaubung gegen Entfall des Entgeltes während eines pensionsversicherungsfreien Dienstverhältnisses ein Überweisungsbetrag gemäß $§ 308$ Abs. 4 ASVG entrichtet wird."

Witwen(Witwer)pension

"Alle Zeiten, in denen ein bescheidmäßig zuerkannter Anspruch auf eine Witwen(Witwer)- pension nach den Bestimmungen des ASVG, BSVG oder GSVG besteht."

Bezug eines Übergangsgeldes

Pflichtversicherung nach dem BSVG / GSVG (Rehabilitation)

Ausbildungzeit nach einem Hochschulstudium

Neutrale Zeit infolge Krankheit

Neutrale Zeit für

Kindererziehung(-pflege)

"Alle Pflichtversicherungszeiten als Betriebsführer eines auf gemeinsame Rechnung und Gefahr von Ehegatten geführten Betriebes (§ 2a Abs. 1 BSVG), sofern keine Ausnahme gemäß $\S 2$ a Abs. 2 BSVG vorliegt. Es gilt daher der halbe Versicherungswert."

"Alle Zeiten, in denen ein verwaistes Kind einen bescheidmäßig zuerkannten Anspruch auf eine Waisenpension hat (Einfach- oder Doppelwaise)."

"Alle Zeiten, in denen ein bescheidmäßig zuerkannter Anspruch auf Übergangsgeld gemäß $\S 199$ und 306 ASVG, § 164 GSVG oder § 156 BSVG besteht."

"Alle Zeiten, in denen einer Person berufliche Maßnahmen der Rehabilitation gemäß $§ 198$ bzw. § 303 ASVG, § 153 BSVG oder $\S 161$ GSVG gewährt werden." "Alle Zeiten einer Pflichtversicherung gemäß $\S 3$ Abs. 5 GSVG bzw. $§ 2$ Abs. 6 BSVG (im Zusammenhang mit Rehabilitationsmaßnahmen)."

"Alle Zeiten einer Ausbildung nach einem Hochschulstudium, wenn aus diesem Grund nicht ohnehin eine Pflichtversicherung entsteht bzw. die Ausbildung im Lehrinstitut für Dentisten in Wien gegeben war. Diese Zeiten aber nur dann, wenn keine Beiträge bezahlt wurden (siehe Kapitel D.6.H4.)."

Versicherungszeit bis einschließlich 31.12 .1972 , die als Ordensangehöriger erworben wurde.

"Alle Zeiten einer Beschäftigung vor der Versicherungspflicht (Beschäftigung im Betrieb der Eltern, Groß-, Wahl- oder Stiefeltern, Pecher, Bergführer usw.), die im Sinne der einschlägigen gesetzlichen Bestimmungen durch Pauschalabgeltung in der Pensionsversicherung zur Anrechnung kommen."

"Alle Zeiten einer Ausbildung am Lehrinstitut für Dentisten, wenn keine Beiträge bezahlt wurden (siehe Kapitel D.6.H5.)."

"Bis einschließlich 31.12.1992 wurde die Sondernotstandshilfe wie das Arbeitslosengeld und die Notstandshilfe unter der gemeinsamen Qualifikation 38 gemeldet. Ab Jänner 1993 ist die Sondernotstandshilfe nicht mehr pfändbar und daher unterscheidbar von den beiden anderen Geldleistungen unter Qualifikation 81 gespeichert."

"Versicherungszeit gemäß ASVG, BSVG und GSVG aufgrund eines rechtskräftigen Entschädigungsanspruches gegenüber einem österreichischen Gericht für eine strafrechtliche Anhaltung oder Verurteilung, wenn mangels gesetzlicher Voraussetzungen eine Beitragsentrichtung nicht gegeben war."

"Alle Zeiten, für die ein Krankenversicherungsträger nach dem ASVG die Versicherungspflicht noch nicht zweifelsfrei festgestellt hat (laufendes Verwaltungsverfahren)."

"Neutrale Zeit infolge Krankheit, wenn nicht aufgrund gesetzlicher Bestimmungen eine Teilpflichtversicherungszeit vorliegt (siehe Kapitel D.6.36.)."

"Neutrale Zeit nach § 234 (1) Z 11 ASVG für Zeiten der Kindererziehung(-pflege) die zur Selbst- oder Weiterversicherung oder zum nachträglichen Einkauf von Versicherungszeiten aus gleichem Grund berechtigt hätte."

Continued on next page... 
... table 1 continued

\begin{tabular}{llll}
\hline \hline qual & type & description & \\
\hline 96 & PV-Pflichtversicherung & nach & "Alle Pflichtversicherungszeiten von Personen, die hauptberuflich im land-
\end{tabular}
dem BSVG - Ehepartner (forst-)wirtschaftlichen Betrieb der Ehegatten beschäftigt sind, sofern keine Betriebsführung auf gemeinsame Rechnung und Gefahr der Ehegatten vorliegt und auch keine Ausnahme aus der Pflichtversicherung nach dem BSVG gegeben ist ( 2a Abs. 1 BSVG)." (forst-)wirtschaftlichen Betrieb der Ehegatten beschäftigt sind, sofern keine Be(halber Versicherungswert) triebsführung auf gemeinsame Rechnung und Gefahr der Ehegatten vorliegt und auch keine Ausnahme aus der Pflichtversicherung nach dem BSVG gegeben ist ( 2a Abs. 1 Z 3 BSVG). Es gilt der halbe Versicherungswert." Doppelbeschäftigung) "Alle Pflichtversicherungszeiten nach dem BSVG für die Angehörigen von Personen, die auf eigene Rechnung und Gefahr einen land-(forst-)wirtschaftlichen Betrieb führen, wenn sie in zwei Betrieben gleichzeitig tätig sind."

A1 PV-Pflichtversicherung - Bezug einer Beihilfe gem. § 20 Abs. 2 lit. c AMFG - Arbeiter Arbeitslosenversicherung

A3 Bezug einer Teilzeitbeihilfe "Alle Zeiten, in denen eine Beihilfe zur Deckung des Lebensunterhaltes gemäß 20 Abs. 2 lit.c des AMFG bezogen wird, wenn die damit verbundene Umschulung für eine Tätigkeit als Arbeiter vorgenommen wird."

"Zeiten, in denen Dienstnehmer gemäß § 1 Abs. 1 ALVG auch dann arbeitslosenversichert sind, wenn sie Anspruch auf Leistungen einer Krankenfürsorgeanstalt haben (z.B. "definitiv gestellte" Dienstnehmer der Bank Austria)."

A4 PV-Pflichtversicherung - Bezug einer Beihilfe gem. § 20 Abs. 2 lit. c AMFG - Angestellter Alle Zeiten eines Bezuges von Teilzeitbeihilfe gemäß § 1 Z 2 Karenzgeldgesetz. "Alle Zeiten, in denen eine Beihilfe zur Deckung des Lebensunterhaltes gemäß 20 Abs. 2 lit.c des AMFG bezogen wird, wenn die damit verbundene Umschulung für eine Tätigkeit als Angestellter vorgenommen wird." h ASVG h ASVG stgeber gem. $\S \S 564$ und 314a ASVG dem BSVG - (Schwieger-)Kind

PV-Pflichtversicherung nach dem BSVG - (Schwieger-)Kind bei Doppelbeschäftigung

Pflichtversicherung nach dem ASVG - Lehrlinge (Arb.)

Pflichtversicherung nach dem ASVG - Lehrlinge (Arb.) mit wesentlich bergmännischer Tätigkeit Pflichtversicherung nach dem ASVG - Lehrlinge (Ang.) mehrfacher geringfügiger Beschäftigung - Arbeiter

"Zeiten, in denen eine Person (Arbeiter) keinen Anspruch auf Arbeitslosengeld hat, weil das erzielte Einkommen aus einer vorübergehenden Beschäftigung den Grenzwert für die Gewährung von Arbeitslosengeld überschritten hat."

"Zeiten, in denen eine Person (Angestellter) keinen Anspruch auf Arbeitslosengeld hat, weil das erzielte Einkommen aus einer vorübergehenden Beschäftigung den Grenzbetrag für die Gewährung von Arbeitslosengeld überschritten hat."

"Alle Zeiten eines pensionsversicherungsfreien Dienstverhältnisses für die ein Pensionsversicherungsträger nach dem Ausscheiden eines Versicherten aus diesem, vom Dienstgeber einen Überweisungsbetrag gemäß §§ 564 und 314a ASVG erhält." "Alle Pflichtversicherungszeiten als (Schwieger-)Kind im land-(forst)wirtschaftlichen Betrieb der Schwiegermutter (des Schwiegervaters); 18. Novelle zum BSVG."

"Alle Pflichtversicherungszeiten als (Schwieger)Kind im land-(forst)wirtschaftlichen Betrieb der Schwiegermutter (des Schwiegervaters), wenn bereits eine Pflichtversicherung als (Schwieger)Kind (Qualifikation A8) vorliegt (Doppelbeschäftigung); 18. Novelle zum BSVG."

"Alle Pflichtversicherungszeiten als Lehrling in der Pensionsversicherung der Arbeiter einschließlich der Pflichtversicherungszeiten als Lehrling in der knappschaftlichen Pensionsversicherung - Arbeiter (bis 31.12.2004), mit Ausnahme der Sonderfälle mit Qualifikation B2."

"Alle Pflichtversicherungszeiten als Arbeiterlehrling in der knappschaftlichen Pensionsversicherung für Personen mit wesentlich bergmännischer Tätigkeit (lt. Anlage 9 zum ASVG)."

"Alle Pflichtversicherungszeiten als Lehrling in der Pensionsversicherung der Angestellten einschließlich der Pflichtversicherungszeiten als Lehrling in der knappschaftlichen Pensionsversicherung - Angestellte."

"Zeiten einer Vollversicherung aufgrund mehrfacher geringfügiger Beschäftigung als Arbeiter nach dem ASVG bzw. B-KUVG, sofern die Geringfügigkeitsgrenze infolge mehrerer geringfügiger Beschäftigungen des Versicherten überschritten wurde oder parallel zu einer geringfügigen Beschäftigung bereits eine Vollversicherung nach dem ASVG bzw. B-KUVG vorliegt. Zeiten eines Krankengeldbzw. Wochengeldbezuges, deren Anspruch aus dieser Vollversicherung resultiert, sind unter der gleichen "Dienstgeberkontonummer" mittels den Qualifikationen 36 und 34 zu melden." 


\begin{tabular}{|c|c|}
\hline$\overline{\text { qual }}$ & type \\
\hline B9 & $\begin{array}{lr}\text { Vollversicherung } & \text { aufgrund } \\
\text { mehrfacher } & \text { geringfügiger } \\
\text { Beschäftigung - Angestellter }\end{array}$ \\
\hline BB & $\begin{array}{l}\text { Vollversicherung aufgrund } \\
\text { mehrfacher geringfügiger } \\
\text { Beschäftigung nach dem B- } \\
\text { KUVG - Beamter/in bzw. } \\
\text { Mandatar/in }\end{array}$ \\
\hline $\mathrm{BD}$ & $\begin{array}{lr}\text { Vollversicherung } & \text { aufgrund } \\
\text { mehrfacher } & \text { geringfügiger } \\
\text { Beschäftigung - } & \text { Dienstleis- } \\
\text { tungsscheck (DLS) } & \end{array}$ \\
\hline
\end{tabular}

description

"Zeiten einer Vollversicherung aufgrund mehrfacher geringfügiger Beschäftigung als Angestellter nach dem ASVG bzw. B-KUVG, sofern die Geringfügigkeitsgrenze infolge mehrerer geringfügiger Beschäftigungen des Versicherten überschritten wurde oder parallel zu einer geringfügigen Beschäftigung bereits eine Vollversicherung nach dem ASVG bzw. BKUVG vorliegt. Zeiten eines Krankengeld- bzw. Wochengeldbezuges, deren Anspruch aus dieser Vollversicherung resultiert, sind unter der gleichen "Dienstgeberkontonummer" mittels den Qualifikationen 36 und 34 zu melden."

"Zeiten einer Vollversicherung aufgrund mehrfacher geringfügiger Beschäftigung nach dem BKUVG, sofern die Geringfügigkeitsgrenze infolge mehrerer geringfügiger Beschäftigungen des Versicherten überschritten wurde oder parallel zu einer geringfügigen Beschäftigung bereits eine Vollversicherung nach dem BKUVG vorliegt."

"Zeiten einer Vollversicherung aufgrund mehrfacher geringfügiger Beschäftigung im Rahmen eines Dienstleistungsschecks, sofern die Geringfügigkeitsgrenze infolge mehrerer geringfügiger Beschäftigungen des Versicherten überschritten wurde oder parallel zu einer geringfügigen Beschäftigung bereits eine Vollversicherung nach dem ASVG bzw. B-KUVG vorliegt. Zeiten eines Krankengeld- bzw. Wochengeldbezuges, deren Anspruch aus dieser Vollversicherung resultiert, sind unter der gleichen "Dienstgeberkontonummer" mittels den Qualifikationen 36 und 34 zu melden."

BE Pflichtversicherung in der PV aufgrund eines Dienstleistungsschecks (DLS)

"Arbeitnehmer, die - ohne berücksichtigungswürdigende Gründe - einen Dienstleistungsscheck verspätet vorlegen und dadurch deren Einkommen im betroffenen Monat die Geringfügigkeitsgrenze übersteigt. In diesem Fall entsteht eine Pflichtversicherung in der Pensionsversicherung jedoch nicht in der Krankenversicherung."

BK $\begin{array}{ll}\text { Bezug von Kombilohn } \\ \text { (Bezugszeiten) }\end{array}$

le Zeiten, in denen zur Förderung der Beschäftigungsaufnahme von länger als ein Jahr langzeitbeschäftigungslosen Personen unter 25 sowie über 45 Jahren im Niedriglohnsektor Kombilohnbeihilfen im Sinne des § 34a AMSG vorliegen. Die Beihilfe an den Arbeitnehmer gilt im Rahmen der Sozialversicherung als Beihilfe zur Deckung des Lebensunterhaltes."

C1 PV-Pflichtversicherung nach dem ASVG - Hausgehilfen Beitragsrückerstattung gem. § 70 Abs. 4 ASVG

Rückerstattung von Krankenversicherungbeiträgen

PV-Pflichtversicherung nach dem ASVG - Hausangestellte knappschaftliche PV) dem ASVG (Angestellte und knappschaftliche PV)

"Alle Pflichtversicherungszeiten auf Grund einer Beschäftigung, die ganz oder teilweise nach dem Hausgehilfen- und Hausangestelltengesetz (HGHAG 1962) geregelt ist. Ausgenommen sind Sonderfälle mit Qualifikation C4."

"Als Kennzeichnung, dass in einem Jahr Pensionsversicherungsbeiträge, welche aufgrund eines Anrechnungsbeitrages gemäß $\S 13$ Bundesbezügegesetz geleistet wurden, wegen Überschreitung der Höchstbeitragsgrundlage rückerstattet wurden, wird vom erstattenden Versicherungsträger ein eintägiges Versicherungsverhältnis (immer der jeweils 1.1. eines Jahres) gemeldet."

"Als Kennzeichnung, dass in einem Jahr Krankenversicherungsbeiträge wegen Überschreitung der Höchstbeitragsgrundlage rückerstattet wurden, wird vom erstattenden Versicherungsträger ein eintägiges Versicherungsverhältnis (immer der jeweils 1.1. eines Jahres) gemeldet."

"Alle Pflichtversicherungszeiten aufgrund einer Beschäftigung, die ganz oder teilweise nach dem Hausgehilfen- und Hausangestelltengesetz (HGHAG 1962) geregelt ist, wenn Dienste höherer Art geleistet werden (Hausangestellte)."

"Alle Zeiten ab 1.1.1998 in denen ein Bezug von Notstands- oder Überbrückungshilfe vorliegt, bzw. ab 1.1.2004 Bezieher einer Beihilfe zur Deckung des Lebensunterhaltes gem. $\S 20$ Abs. 2 lit. c des AMFG (DLU) im Zusammenhang mit Notstands- oder Überbrückungshilfe."

"Alle Pflichtversicherungszeiten in der Pensionsversicherung der Arbeiter (knappschaftliche PV) nach dem ASVG (OMV-Mitarbeiter) ab dem 1. Juli 1993."

"Alle Pflichtversicherungszeiten in der Pensionsversicherung der Angestellten (knappschaftliche PV) nach dem ASVG ab dem 1. Juli 1993 (OMV-Mitarbeiter)."

"Als Kennzeichnung, dass in einem Jahr Pensionsversicherungsbeiträge wegen Überschreitung der Höchstbeitragsgrundlage rückerstattet wurden, wird vom erstattenden Versicherungsträger ein eintägiges Versicherungsverhältnis (immer der jeweils 1.1. eines Jahres) gemeldet."

"Alle Zeiten, für die bei der Sozialversicherungsanstalt der gewerblichen Wirtschaft und der Sozialversicherungsanstalt der Bauern Beiträge wegen Vorliegens einer anderen Beschäftigung (Mehrfachversicherung) nicht bzw. nur zum Teil vorgeschrieben werden." 


\begin{tabular}{llll}
\hline \hline qual & type & description \\
\hline CX & Rückerstattung & von Arbeit- & "Als Kennzeichnung, dass in einem Jahr Arbeitslosenversicherungsbeiträge wegen \\
& slosenversicherungsbeiträgen & Überschreitung der Höchstbeitragsgrundlage im Zuge der Mehrfachversicherung \\
& rückerstattet wurden, ist vom erstattenden Versicherungsträger ein eintägiges \\
& Versicherungsverhältnis (immer der jeweils 1.1 eines Jahres) zu melden. Gemäß \\
& $\S 45$ AIVG sind die jeweiligen krankenversicherungsrechtlichen Vorschriften mit \\
& der Maßgabe anzuwenden, dass an die Stelle der Krankenversicherung die Ar- \\
& beitslosenversicherung tritt. § 70 ASVG ist überdies mit der Maßgabe anzuwen- \\
& den, dass an die Stelle des dort genannten Prozentsatzes des Erstattungsbetrages \\
& der für den von der (dem) Versicherten zu tragenden Anteil am Arbeitslosenver- \\
& sicherungsbeitrag geltenden Prozentsatz tritt."
\end{tabular}

D1 PV-Pflichtversicherung nach "Alle Pflichtversicherungszeiten in der Pensionsversicherung der Arbeiter aufdem ASVG - Hausbesorger grund eines privatrechtlichen Dienstverhältnisses als Hausbesorger (HBG 1970)."

D2 Pflichtversicherung aufgrund "Alle Zeiten, für die eine Urlaubsentschädigung bzw. eine Ersatzleistung für einer Urlaubsabfindung oder Urlaubsentgelt (Urlaubsentschädigung, Urlaubsabfindung) gewährt wird."

-entschädigung

D3 Pflichtversicherung

aufgrund einer

Kündigungsentschädigung

Pflichtversicherung auf-

grund einer Winter-

feiertagsentschädigung

sicherungsdauer

"Alle Zeiten, für die von der Bauarbeiter-Urlaubskasse WinterBezug einer vorzeitiger Alterspension wegen geminderter Erwerbsfähigkeit ASVG

Selbstversicherung gem. $\S 16 a$ dem ASVG für Zeiten der Kindererziehung (-pflege) (Arb. und knappschaft. PV) schaft. PV)

Continued on next page...

Alle Zeiten, in denen ein bescheidmäßig zuerkannter Anspruch auf eine vorzeitige Alterspension bei Arbeitslosigkeit besteht."

"Alle Zeiten, in denen ein bescheidmäßig zuerkannter Anspruch auf eine vorzeitige Alterspension wegen geminderter Erwerbsfähigkeit besteht."

"Alle Zeiten, in denen ein normaler Alterspensionsbezug vorliegt und der Pensionist (die Pensionistin) weiterhin ein sozialversicherungspflichtiges Einkommen bezieht, welches eine bestimmte Höchstgrenze überschreitet."

"Alle Zeiten, in denen ein bescheidmäßig zuerkannter Anspruch auf eine Gleitpension besteht. Ab 1.1.1998 sind unter dieser Qualifikation nur Gleitpensionen gemäß $\S 253 \mathrm{c}$ Abs. $1 \mathrm{Z} 1$ lit.a ASVG, § 131b Abs. 1 Z1 lit.a GSVG und § 122b Abs. 1 Z 1 lit.a BSVG zu speichern (siehe auch Kapitel D.6.N9.)."

"Alle Zeiten der Pflege eines im gemeinsamen Haushalt lebenden behinderten Kindes, für das eine erhöhte Familienbeihilfe im Sinne des § 8 Abs. 4 des Familienlastenausgleichsgesetzes 1967 gewährt wird, wenn die Arbeitskraft der pflegenden Person aus diesem Grund gänzlich beansprucht wird."

Alle Zeiten einer Selbstversicherung in der Pensionsversicherung gemäß $\S 16 \mathrm{a}$ ASVG.

Alle Zeiten einer Kindererziehung pro Kind maximal 48 Monate beginnend ab dem Monatsersten nach Vorliegen der Voraussetzungen." 1.1.1979 von einer Entwicklungshilfeorganisation gemäß $§ 1$ Abs. 2 des Entwicklungshilfegesetzes im Rahmen der Entwicklungshilfe als Entwicklungshelfer oder Experte beschäftigt bzw. ausgebildet werden, Beiträge entrichtet werden."

"Zeiten, für die Beiträge zur freiwilligen Weiterversicherung in der Pensionsversicherung nach dem ASVG zum Zwecke des nachträglichen Einkaufes von Versicherungszeiten für Zeiten der Kindererziehung (-pflege) bis 31.12.1978 gemäß Art. VII des Sozialrechtsänderungsgesetzes 1978 (33. Novelle zum ASVG) entrichtet werden."

"Zeiten, für die in der Pensionsversicherung der Arbeiter bzw. in der knappschaftlichen Pensionsversicherung als Arbeiter Beiträge zur freiwilligen Weiterversicherung wegen Kindererziehung (-pflege) entrichtet werden."

"Zeiten, für die in der Pensionsversicherung der Angestellten bzw. in der knappschaftlichen Pensionsversicherung als Angestellte Beiträge zur freiwilligen Weiterversicherung wegen Kindererziehung (-pflege) entrichtet werden." 


\begin{tabular}{ll}
\hline \hline qual & type \\
\hline E8 & Selbstversicherung nach dem \\
& ASVG für Zeiten der Kinder- \\
& erziehung (-pflege) (Arb. und \\
& knappschaft. PV) \\
E9 & Selbstversicherung nach dem \\
& ASVG für Zeiten der Kinder- \\
& erziehung (-pflege) (Ang. und \\
knappschaft. PV) \\
Vorläufige Versicherungszeit \\
wegen Kindererziehung
\end{tabular}

description

"Zeiten, für die in der Pensionsversicherung der Arbeiter bzw. in der knappschaftlichen Pensionsversicherung als Arbeiter Beiträge zur Selbstversicherung nach § 18 ASVG wegen Kindererziehung (-pflege) entrichtet werden."

"Zeiten, für die in der Pensionsversicherung der Angestellten bzw. in der knappschaftlichen Pensionsversicherung als Angestellte Beiträge zur Selbstversicherung nach $\S 18$ ASVG wegen Kindererziehung (-pflege) entrichtet werden."

"Im Rahmen des Pensionskontos ist es erforderlich, "fiktive", d.h. noch nicht geprüfte Kindererziehungszeiten an die Versicherungsdatei melden zu können. Zu diesem Zweck dient obgenannte Qualifikation. Basis sind Meldungen des Kinderbetreuungsgeldkompetenzcenter, die über die Datendrehscheibe des Hauptverbandes an den zuständigen Pensionsversicherungsträger übermittelt werden. Im Zuge der Leistungsfeststellung von Seiten des PV-Trägers werden diese Zeiten geprüft und unter der Qualifikation "E3" in die Versicherungsdatei eingespeichert. Es dürfen pro Kind maximal 60 Monate gemeldet werden."

F1 Pflichtversicherung nach dem FSVG - freiberuflich selbständige Erwerbstätigkeit

Pflichtversicherung nach dem GSVG - mit gleichzeitiger Pflichtversicherung aufgrund freiberuflich sebständiger Erwerbstätigkeit (FSVG)

Pflichtversicherung gem. Abs.1 Z.4 GSVG

gem. 2 Abs.1 Z 4 GSVG mit gleichzeitiger Pflichtversicherung aufgrund freiberuflich selbständiger Erwerbstätigkeit (FSVG)

Nachträglicher Einkauf von Versicherungszeiten aufgrund freiberuflich selbständiger Erwerbstätigkeit (FSVG)

Freiwillige Weiterversicherung nach dem BSVG - Angehöriger mit halber Mindestbeitragsgrundlage

Freiwillige Weiterversicherung nach dem BSVG - Ehegatte (halbe Mindestbeitragsgrundlage)

F8 Freiwillige Weiterversicherung nach dem BSVG - Betriebsführer (halbe Mindestbeitragsgrundlage)

Freiwillige Weiterversicherung

"Zeiten, für die Beiträge zur Pflichtversicherung ab 1.1.1979 nach den Bestimmungen des Bundesgesetzes (FSVG) vom 30.11.1978 über die Sozialversicherung freiberuflich selbständig Erwerbstätiger entrichtet werden. Diese Meldung impliziert in weiterer Folge, dass die betroffene Person in ihrer Eigenschaft als Angehöriger keinen KV-Anspruch haben kann. Da es sich hierbei um eine PVQualifikation handelt, begründet diese Meldung auch keinen Eigenanspruch in der Krankenversicherung."

"Zeiten, für die Beiträge zur Pflichtversicherung nach dem GSVG mit gleichzeitiger Pflichtversicherung wegen freiberuflich selbständiger Erwerbstätigkeit ab dem 1.1.1979 nach den Bestimmungen des Gesetzes vom 30.11.1978 über die Sozialversicherung freiberuflich selbständig Erwerbstätiger entrichtet werden. Diese Meldung impliziert in weiterer Folge, dass die betroffene Person in ihrer Eigenschaft als Angehöriger keinen KV-Anspruch haben kann. Da es sich hierbei um eine PV-Qualifikation handelt, begründet diese Meldung auch keinen Eigenanspruch in der Krankenversicherung."

Zeiten einer selbständigen Erwerbstätigkeit gemäß $§ 2$ Abs. 1 Z 4 GSVG.

"Zeiten einer selbständigen Erwerbstätigkeit gemäß $§ 2$ Abs.1 Z 4 GSVG mit gleichzeitiger Pflichtversicherung wegen freiberuflich selbständiger Erwerbstätigkeit ab 1.1.1998. Diese Meldung impliziert in weiterer Folge, dass die betroffene Person in ihrer Eigenschaft als Angehöriger keinen KV-Anspruch haben kann. Da es sich hierbei um eine PV-Qualifikation handelt, begründet diese Meldung auch keinen Eigenanspruch in der Krankenversicherung."

"Zeiten, für die Beiträge der freiwilligen Weiterversicherung zum Zwecke des nachträglichen Einkaufes bis 31.12.1978 aufgrund freiberuflich selbständiger Erwerbstätigkeit nach den Bestimmungen des Bundesgesetzes (FSVG) vom 30.11.1978 über die Sozialversicherung freiberuflich selbständig Erwerbstätiger entrichtet wurden."

"Alle Zeiten von Personen, die nach dem Ausscheiden aus einer Pflichtversicherung als Angehöriger nach dem BSVG, Beiträge zur freiwilligen Weiterversicherung in der Pensionsversicherung nach dem BSVG entrichten."

"Alle Zeiten von Personen, die nach dem Ausscheiden aus einem von den Ehepartnern auf gemeinsame Rechnung und Gefahr geführten land-(forst)wirtschaftlichen Betriebes, Beiträge zur freiwilligen Weiterversicherung in der Pensionsversicherung nach dem BSVG entrichten."

"Alle Zeiten von Personen, die nach dem Ausscheiden aus einer Beschäftigung als Betriebsführer eines auf gemeinsame Rechnung und Gefahr der Ehegatten geführten land- (forst-) wirtschaftlichen Betriebes Beiträge zur freiwilligen Weiterversicherung in der Pensionsversicherung nach dem BSVG entrichten. Für diese Personen gilt für den Mindestbeitrag die halbe Mindestbeitragsgrundlage."

"Alle Zeiten von Personen, die nach dem Ausscheiden aus einer Beschäftigung als nach dem BSVG - Ehegatte hauptberuflich tätiger Ehegatte im land-(forst-)wirtschaftlichen Betrieb des Ehegatten, Beiträge zur freiwilligen Weiterversicherung in der Pensionsversicherung nach dem BSVG entrichten."

Continued on next page.. 


\begin{tabular}{|c|c|c|}
\hline qual & type & description \\
\hline$\overline{\mathrm{G} 1}$ & $\begin{array}{l}\text { PV-Pflichtversicherung nach } \\
\text { dem ASVG/NSchG (Arb. und } \\
\text { knappschaft. PV) }\end{array}$ & $\begin{array}{l}\text { "Alle Pflichtversicherungszeiten in der Pensionsversicherung der Arbeiter nach } \\
\text { dem ASVG, bei Vorliegen einer Beschäftigung gem. dem NSchG, einschließlich } \\
\text { der Pflichtversicherungszeiten als Arbeiter in der knappschaftlichen Pensionsver- } \\
\text { sicherung (bis 31.12.2004) mit Ausnahme der Sonderfälle mit Qualifikation G2, } \\
\text { G3." }\end{array}$ \\
\hline G2 & $\begin{array}{l}\text { PV-Pflichtversicherung } \text { nach } \\
\text { dem ASVG/NSchG (knapp- } \\
\text { schaft. PV, Arb. mit } \\
\text { wesentlich } \quad \text { bergmännischer } \\
\text { Tätigkeit) }\end{array}$ & $\begin{array}{l}\text { "Alle Pflichtversicherungszeiten als Arbeiter in der knappschaftlichen Pen- } \\
\text { sionsversicherung für Personen mit wesentlich bergmännischer Tätigkeit, wenn } \\
\text { eine Beschäftigung gem. dem NSchG vorliegt." }\end{array}$ \\
\hline G3 & $\begin{array}{l}\text { PV-Pflichtversicherung nach } \\
\text { dem ASVG/NSchG (knapp- } \\
\text { schaft. PV, Arb. mit } \\
\text { Gewinnhauertätigkeit) }\end{array}$ & $\begin{array}{l}\text { "Alle Pflichtversicherungszeiten als Arbeiter in der knappschaftlichen } \\
\text { Pensionsversicherung für Personen mit Gewinnhauertätigkeit, wenn eine } \\
\text { Beschäftigung gem. dem NSchG vorliegt." }\end{array}$ \\
\hline G4 & $\begin{array}{l}\text { PV-Pflichtversicherung nach } \\
\text { dem ASVG/NSchG (Ang. und } \\
\text { knappschaftl. PV) }\end{array}$ & $\begin{array}{l}\text { "Alle Pflichtversicherungszeiten in der Pensionsversicherung der Angestellten ein- } \\
\text { schließlich der Pflichtversicherungszeiten als Angestellter in der knappschaftlichen } \\
\text { Pensionsversicherung (bis } 31.12 .2004 \text { ), mit Ausnahme der Sonderfälle mit Quali- } \\
\text { fikation G5, G6." }\end{array}$ \\
\hline G5 & $\begin{array}{l}\text { PV-Pflichtversicherung nach } \\
\text { dem ASVG/NSchG (knapp- }\end{array}$ & $\begin{array}{l}\text { "Alle Pflichtversicherungszeiten als Angestellter in der knappschaftlichen Pen- } \\
\text { sionsversicherung für Personen mit wesentlich bergmännischer Tätigkeit." }\end{array}$ \\
\hline
\end{tabular}
sionsversicherung für Personen mit wesentlich bergmännischer Tätigkeit."

schaft. PV, Ang. mit wesentlich bergmännischer Tätigkeit) dem ASVG/NSchG (knappschaft. PV, Ang. mit Gewinnhauertätigkeit) DLS

"Alle Pflichtversicherungszeiten als Angestellter in der knappschaftlichen Pensionsversicherung für Personen mit Gewinnhauertätigkeit (lt. Anlage 10 zum ASVG)."

"Schüler (Schülerinnen), die in Ausbildung zum gehobenen Dienst für Gesundheits- und Krankenpflege nach dem Gesundheits- und Krankenpflegegesetz, BGBl. I Nr. 108/97, oder zum Krankenpflegedienst oder zum medizinischtechnischen Fachdienst im Sinne des Bundesgesetzes über die Regelung des medizinisch-technischen Fachdienstes und der Sanitätshilfsdienste, BGBl. Nr. 102/1961, stehen, bzw. Studierende an einer medizinischtechnischen Akademie nach dem MTD-Gesetz, BGBl. Nr. 460/1992, oder an einer Hebammenakademie nach dem Hebammengesetz, BGBl. Nr. 310/1994, (§ 4 Abs. 1 Z 5 ASVG). Bis 31.12.1997 war dieser Personenkreis unter der Qualifikation 14 gespeichert."

"Alle Zeiten, in denen eine Beschäftigung nach dem ASVG bzw. B-KUVG als Arbeiter(in) vorliegt, wenn der jeweilige Richtsatz für die Geringfügigkeit nicht überschritten wird. Nur Unfallversicherung!"

"Alle Zeiten, in denen eine Beschäftigung nach dem ASVG bzw. B-KUVG als Angestellte(r) vorliegt, wenn der jeweilige Richtsatz für die Geringfügigkeit nicht überschritten wird. Nur Unfallversicherung!"

"Alle Zeiten, in denen eine Beschäftigung im Rahmen eines Dienstleistungsschecks vorliegt, wenn der jeweilige Richtsatz für die Geringfügigkeit nicht überschritten wird. Nur Unfallversicherung!"

"Alle Zeiten, in denen eine Beschäftigung nach dem B-KUVG als Beamter/in oder Mandatar/in vorliegt, wenn der jeweilige Richtsatz für die Geringfügigkeit nicht überschritten wird. Nur Unfallversicherung!"

"Alle Zeiten des Besuches einer inländischen öffentlichen oder mit dem Öffentlichkeitsrecht ausgestatteten mittleren Schule mit mindestens zweijährigem Bildungsgang, wobei erstmalig jenes Schuljahr gemeldet wird, das in dem Kalenderjahr beginnt, in dem der Schüler das 15. Lebensjahr vollendet, sofern auf Grund der 44. Novelle zum ASVG, der 11. Novelle zum BSVG bzw. der 13. Novelle zum GSVG Beiträge entrichtet wurden und diese Zeiten daher gemäß § 227 Abs. 3 ASVG, § 107 Abs. 8 BSVG bzw. § 116 Abs. 8 GSVG leistungswirksam sind."

"Alle Zeiten des Besuches einer inländischen höheren Schule, Akademie oder verwandten Lehranstalt in dem für diese Schularten vorgeschriebenen normalen Ausbildungsgang, wobei erstmalig jenes Schuljahr gemeldet wird, das in dem Kalenderjahr beginnt, in dem der Schüler das 15. Lebensjahr vollendet. Alle diese Zeiten nur dann, wenn aufgrund der 44. Novelle zum ASVG, der 11. Novelle zum BSVG bzw. der 13. Novelle zum GSVG Beiträge entrichtet wurden und diese Zeiten daher gemäß § 227 Abs. 3 ASVG, § 107 Abs. 8 BSVG bzw. § 116 Abs. 8 GSVG leistungswirksam sind." 


\begin{tabular}{|c|c|c|}
\hline qual & type & description \\
\hline H3 & $\begin{array}{l}\text { Ausbildung nach einem } \\
\text { Hochschulstudium } \\
\text { Beitragszahlung }\end{array}$ & 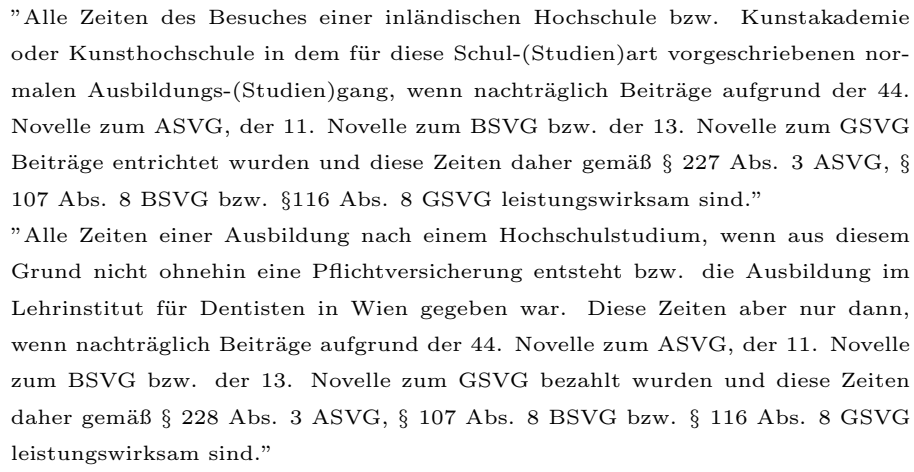 \\
\hline $\mathrm{HB}$ & $\begin{array}{l}\text { Höhere Schule, } \\
\text { Beitragszahlung (Beamte) }\end{array}$ & $\begin{array}{l}\text { "Gemäß } \S 53 \text { Abs. } 2 \text { lit. h PG1965, } 46 \text { Abs. } 2 \text { lit. h BB-PG sind die } \\
\text { Zeit eines abgeschlossenen inländischen oder einem solchen gleichzuhaltenden } \\
\text { Studiums an einer öffentlichen oder mit dem Öffentlichkeitsrecht ausgestatteten } \\
\text { höheren Schule, soweit die gesetzliche Mindestdauer nicht überschritten worden } \\
\text { ist, anzurechnen. Die zwingende Meldung einer Beitragsgrundlage ist vorgesehen, } \\
\text { da die Höhe dieser Beitragsgrundlage variiert. Für alle ab } 1955 \text { geborenen Bundes- } \\
\text { beamtInnen ist das Allgemeine Pensionsgesetz (APG) anzuwenden. Diese Zeiten- } \\
\text { und Beitragsgrundlagenspeicherung dient primär zur Befüllung des einheitlichen } \\
\text { Pensionskontos (ePK)." }\end{array}$ \\
\hline I1 & $\begin{array}{l}\text { Selbstversicherung } \\
\text { ASVG }\end{array}$ & $\begin{array}{l}\text { "Alle Zeiten, für die Beiträge zur Selbstversicherung in der Krankenversicherung } \\
\text { gemäß } § 16 \text { Abs. } 1 \text { ASVG entrichtet werden." }\end{array}$ \\
\hline $\mathrm{I} 2$ & $\begin{array}{l}\text { Selbstversicherung } \\
\text { ASVG }\end{array}$ & $\begin{array}{l}\text { "Alle Zeiten, für die Beiträge zur Selbstversicherung in der Krankenversicherung } \\
\text { gemäß } § 16 \text { Abs. } 2 \text { ASVG entrichtet werden." }\end{array}$ \\
\hline I3 & $\begin{array}{l}\text { Selbstversicherung } \S 16 \text { ASVG } \\
\text { - Wartezeit }\end{array}$ & $\begin{array}{l}\text { "Alle Zeiten, für die Beiträge zur Selbstversicherung in der Krankenversicherung } \\
\text { gemäß } § 16 \text { entrichtet werden und gemäß den Bestimmungen des } \S 124 \text { ASVG kein } \\
\text { Leistungsanspruch besteht." }\end{array}$ \\
\hline $\mathrm{I} 4$ & $\begin{array}{l}\text { Krankenversicherung bei Pen- } \\
\text { sionsbezug }\end{array}$ & $\begin{array}{l}\text { "Alle Zeiten, die auf Grund eines Pensionsbezuges - ausgenommen nach dem } \\
\text { GSVG und BSVG - einen Krankenversicherungsschutz ergeben." }\end{array}$ \\
\hline I5 & $\begin{array}{l}\text { Bezug von Kinderbetreuungs- } \\
\text { geld }\end{array}$ & $\begin{array}{l}\text { "Unter oben angeführter Qualifikation sind jene Zeiten zu melden, in denen der } \\
\text { Versicherte Kinderbetreuungsgeld nach dem KBGG bezieht. Gemäß } \S 12 \text { Abs. } \\
\text { 5a ASVG, } ₹ 7 \text { Abs. 1Z. } 5 \text { GSVG und BSVG endet die Krankenversicherung der } \\
\text { Bezieher von Kinderbetreuungsgeld mit Ablauf des Kalendermonates, für den let- } \\
\text { ztmalig Kinderbetreuungsgeld ausgezahlt wird." }\end{array}$ \\
\hline I7 & $\begin{array}{l}\text { Anspruch auf Kinderbetreu- } \\
\text { ungsgeld }\end{array}$ & $\begin{array}{l}\text { "Diese Qualifikationsmeldung dient zur Feststellung der Anrechnung der Kinder- } \\
\text { erziehung als Beitragszeit in der Pensionsversicherung. Es sind jene Zeiten zu } \\
\text { melden, in denen der Versicherte Anspruch auf Kinderbetreuungsgeld nach dem } \\
\text { KBGG hat, dieser jedoch ausschließlich auf Grund von Wochengeldbezug ruht." }\end{array}$ \\
\hline I8 & $\begin{array}{l}\text { Krankenversicherung bei } \\
\text { Pensionsbezug (auf Dienstge- } \\
\text { berkontonummer bezogen) }\end{array}$ & $\begin{array}{l}\text { "Alle Zeiten, die auf Grund eines Pensionsbezuges - ausgenommen nach dem } \\
\text { GSVG und BSVG - einen Krankenversicherungsschutz ergeben. Im Unterschied } \\
\text { zu der Qualifikation I4 ist hier die Eingabe einer Dienstgeberkontonummer er- } \\
\text { forderlich." }\end{array}$ \\
\hline I9 & $\begin{array}{l}\text { Krankenversicherung bei Er- } \\
\text { werbstätigkeit nach dem BSVG }\end{array}$ & $\begin{array}{l}\text { "Alle Zeiten, die auf Grund einer Erwerbstätigkeit nach dem BSVG einen } \\
\text { Krankenversicherungsschutz ergeben. Diese Meldung erfolgt parallel zu den bere- } \\
\text { its bestehenden pensionsversicherungsrelevanten Qualifikationen" }\end{array}$ \\
\hline IA & $\begin{array}{l}\text { KV-Pflichtversicherung- } \\
\text { Sachleistung wegen KBG } \\
\text { (GSVG) }\end{array}$ & $\begin{array}{l}\text { "Da die Qualifikationen I5 und I7 von der SVA der gewerblichen Wirtschaft } \\
\text { im Bezug auf Kinderbetreuungsgeld ausschließlich als PV-Qualifikationen } \\
\text { gemeldet werden, dient diese Qualifikation zur Meldung eines Krankenver- } \\
\text { sicherungsanspruches nach dem GSVG aufgrund des Bezuges von Kinderbetreu- } \\
\text { ungsgeld mit Sachleistungsanspruch. Besteht die Krankenversicherung aufgrund } \\
\text { des Kinderbetreuungsgeld neben einer anderen Krankenversicherung nach dem } \\
\text { GSVG, wird sie zu den bereits bestehenden Qualifikationen zusätzlich gemeldet." }\end{array}$ \\
\hline IB & $\begin{array}{l}\text { KV-Pflichtversicherung- } \\
\text { Geldleistung wegen KBG } \\
\text { (GSVG) }\end{array}$ & $\begin{array}{l}\text { "Da die Qualifikationen I5 und I7 von der SVA der gewerblichen Wirtschaft } \\
\text { im Bezug auf Kinderbetreuungsgeld ausschließlich als PV-Qualifikationen } \\
\text { gemeldet werden, dient diese Qualifikation zur Meldung eines Krankenver- } \\
\text { sicherungsanspruches nach dem GSVG auf Grund des Bezuges von Kinderbetreu- } \\
\text { ungsgeld mit Geldleistungsanspruch. Besteht die Krankenversicherung auf Grund } \\
\text { des Kinderbetreuungsgeld neben einer anderen Krankenversicherung nach dem } \\
\text { GSVG, wird sie zu den bereits bestehenden Qualifikationen zusätzlich gemeldet." }\end{array}$ \\
\hline $\mathrm{J} 1$ & $\begin{array}{l}\text { Pflichtversicherung } \\
\text { öffentlich Bediensteter }\end{array}$ & $\begin{array}{l}\text { "Alle Zeiten einer pragmatisierten Beschäftigung als öffentlich Bediensteter gemäß } \\
\S 1 \text { Abs. } 1 \text { (ausgenommen Z 7,12 und 14b) des B-KUVG bzw. gemäß } \S 472 \text { oder } \\
479 \text { a ASVG." }\end{array}$ \\
\hline
\end{tabular}

Continued on next page... 
... table 1 continued

\begin{tabular}{llrl}
\hline \hline qual & type & description \\
\hline J2 & Karenzurlaub & mit & "Alle Zeiten eines Karenzurlaubes gemäß $\S 7$ Abs. 2 Z 3 des B-KUVG und \\
& Beitragszahlung & äquivalenter Bestimmungen bei der Versicherungsanstalt der österreichischen \\
& & Eisenbahnen und bei der Betriebskrankenkasse der Wiener Verkehrsbetriebe." \\
J3 & Vertragsbedienstete nach B- & "Alle Zeiten, in denen eine Beschäftigung als Vertragsbediensteter nach dem \\
& KUVG, Einkommen unter der & B-KUVG vorliegt, wenn der jeweilige Richtsatz für Geringfügigkeit nicht \\
& Geringfügigkeitsgrenze gem. $\S$ & überschritten wird. Nur kranken- und unfallversichert!"
\end{tabular}
$5 / 2$ ASVG

Ruhegenuss aufgrund einer "Alle Zeiten eines Anspruches auf Ruhegenuss gemäß $\S 1$ Abs. 1 Z 7, 12 und Beschäftigung als öffentlich 14b des BKUVG und äquivalenter Bestimmungen bei der Versicherungsanstalt für Bediensteter Eisenbahnen und Bergbau und bei der Betriebskrankenkasse der Wiener Verkehrsbetriebe."

Versorgungsgenuss - Witwe "Alle Zeiten in denen ein Versorgungsgenuss - Witwe/Witwer - gemäß $\S 1$ Abs. (Witwer)

$1 \mathrm{Z} \mathrm{7,12}$ oder 14b des B-KUVG und äquivalenter Bestimmungen bei der Versicherungsanstalt für Eisenbahnen Bergbau und bei der Betriebskrankenkasse der Wiener Verkehrsbetriebe vorliegt."

J7 Versorgungsgenuss - Waise "Alle Zeiten in denen ein Versorgungsgenuss - Waise - gemäß $\S 1$ Abs. 1 Z 7, 12 oder 14b des B-KUVG und äquivalenter Bestimmungen bei der Versicherungsanstalt für Eisenbahnen und Bergbau und bei der Betriebskrankenkasse der Wiener Verkehrsbetriebe vorliegt."

Pflichtversicherte nach dem BKUVG - § 4 Versicherte B-KUVG, $\S 1 / 1 / 8$ - 11 B-KUVG ('Politiker')

"Alle Zeiten einer Kranken- und Unfallversicherung für Personen, die gemäß $\S 4$ B-KUVG versichert sind."

Alle Zeiten einer Pflichtversicherung nach dem B-KUVG - § 1 Abs. 1 Ziffer 8-11 ("Politiker")

"Alle Zeiten eines öffentlich-rechtlichen Dienstverhältnisses zum Bund für alle ab 1955 geborenen Bundesbeamten/innen, die dem Pensionsgesetz 1965 unterliegen - ausgenommen sind Zeiten einer Dienstzuteilung nach $\S 17$ Abs. 1a PTSG zur Post AG, Telekom AG und Postbus AG. Für alle ab 1955 geborenen Bundesbeamten/innen ist das Allgemeine Pensionsgesetz (APG) anzuwenden. Diese Zeiten- und Beitragsgrundlagenspeicherung dient primär zur Befüllung des einheitlichen Pensionskontos (ePK)."

"Alle Zeiten nach dem Pensionsgesetz 1965, dem Bundestheaterpensionsgesetz und dem Bundesbahn-Pensionsgesetz für die ein (besonderer) Pensionsbeitrag geleistet wurde und somit in der Beamtenpensionsversicherung anzurechnen sind. Diese Zeiten- und Beitragsgrundlagenspeicherung dienst primär zur Befüllung des einheitlichen Pensionskontos (ePK)."

JM Geprüfte Beamtennullzeit Wurde im Zuge eines Datenergänzungsverfahrens für Beamtenversicherungszeiten festgestellt, dass für einen Zeitraum keine für Beamtenvordienstzeiten vorliegen, so ist dieser Zeitraum als geprüfte Beamtennullzeit zu melden. Diese Zeiten- und Beitragsgrundlagenspeicherung dient primär zur Befüllung des einheitlichen Pensionskontos (ePK)."

PV-Pflichtversicherung als Beamter/in bei den ÖBB

"Alle Zeiten eines öffentlich-rechtlichen Dienstverhältnisses bei den

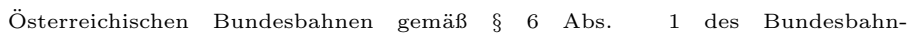
Pensionsgesetzes. Diese Zeiten- und Beitragsgrundlagenspeicherung dient primär zur Befüllung des einheitlichen Pensionskontos (ePK)."

K1 Selbstversicherung in der Krankenversicherung (§ 16 ASVG) ingfügiger Beschäftigung gem. $\S 19$ a ASVG - Arbeiter

"Alle Zeiten, für die Beiträge zur Selbstversicherung in der Krankenversicherung gemäß $§ 16$ ASVG entrichtet werden."

"Zeiten einer Selbstversicherung bei geringfügiger Beschäftigung gemäß $\S 19 \mathrm{a}$ ASVG. Zeiten eines Krankengeld- bzw. Wochengeldbezuges, deren Anspruch aus dieser Selbstversicherung resultiert, sind unter der gleichen "Dienstgeberkontonummer" mittels den Qualifikationen 36 und 34 zu melden."

"Zeiten einer Selbstversicherung bei geringfügiger Beschäftigung gemäß $\S 19 \mathrm{a}$ ASVG. Zeiten eines Krankengeld- bzw. Wochengeldbezuges, deren Anspruch aus dieser Selbstversicherung resultiert, sind unter der gleichen "Dienstgeberkontonummer" mittels den Qualifikationen 36 und 34 zu melden."

"Alle Zeiten, für die Beiträge zur freiwilligen Weiterversicherung in der Pensionsversicherung der Arbeiter, auch als Arbeiter in der knappschaftlichen Pensionsversicherung entrichtet werden, bei denen die Dienstgeberanteile aus Mitteln des Bundes getragen werden ( $\$ 77$ Abs. 6 ASVG)." 


\begin{tabular}{|c|c|c|}
\hline qual & type & description \\
\hline$\overline{\mathrm{K} 5}$ & 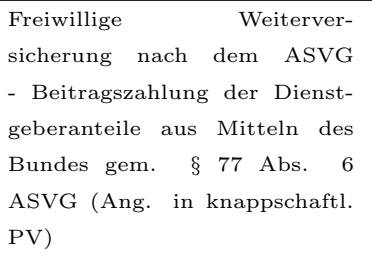 & $\begin{array}{l}\text { "Alle Zeiten, für die Beiträge zur freiwilligen Weiterversicherung in der Pen- } \\
\text { sionsversicherung der Angestellten, auch als Angestellte in der knappschaftlichen } \\
\text { Pensionsversicherung entrichtet werden, bei denen die Dienstgeberanteile aus Mit- } \\
\text { teln des Bundes getragen werden ( } § 77 \text { Abs. } 6 \text { ASVG)." }\end{array}$ \\
\hline K6 & 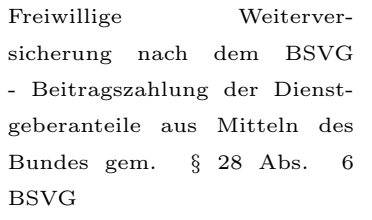 & $\begin{array}{l}\text { "Alle Zeiten, für die Beiträge zur freiwilligen Weiterversicherung nach dem GSVG } \\
\text { entrichtet werden, bei denen die Dienstgeberanteile aus Mitteln des Bundes ge- } \\
\text { tragen werden ( } \$ 33 \text { Abs. } 9 \text { GSVG)." }\end{array}$ \\
\hline K7 & $\begin{array}{l}\text { Freiwillige } \\
\text { sicherung nach dem GSVG } \\
\text { - Beitragszahlung der Dienst- } \\
\text { geberanteile aus Mitteln des } \\
\text { Bundes gem. } \quad \text { \& } 33 \text { Abs. } 9 \\
\text { GSVG }\end{array}$ & $\begin{array}{l}\text { "Alle Zeiten, für die Beiträge zur freiwilligen Weiterversicherung nach dem BSVG } \\
\text { entrichtet werden, bei denen die Dienstgeberanteile aus Mitteln des Bundes ge- } \\
\text { tragen werden ( } \S 28 \text { Abs. } 6 \text { BSVG)." }\end{array}$ \\
\hline K9 & $\begin{array}{llr}\text { Weiterversicherung } & \text { in } & \text { der } \\
\text { Krankenversicherung } \quad(\S \quad 8 \\
\text { BSVG) }\end{array}$ & $\begin{array}{l}\text { "Alle Zeiten, für die Beiträge zur Weiterversicherung in der Krankenversicherung } \\
\text { gemäß } § 8 \text { BSVG entrichtet werden." }\end{array}$ \\
\hline KA & $\begin{array}{l}\text { Selbstversicherung bei ger- } \\
\text { ingfügiger Beschäftigung gem. } \\
\S 7 \text { a Abs. } 2 \text { Z } 1 \text { B-KUVG - } \\
\text { Arbeiter }\end{array}$ & $\begin{array}{l}\text { "Zeiten einer Selbstversicherung als Arbeiter bei geringfügiger Beschäftigung } \\
\text { gemäß } \S 7 \mathrm{a} \text { Abs. } 2 \mathrm{Z} 1 \mathrm{~B}-\mathrm{KUVG} \text {. Zeiten eines Krankengeld- bzw. Wochen- } \\
\text { geldbezuges, deren Anspruch aus dieser Selbstversicherung resultiert, sind unter } \\
\text { der gleichen "Dienstgeberkontonummer" mittels den Qualifikationen } 36 \text { und } 34 \\
\text { zu melden. Diese Qualifikationsmeldung repräsentiert eine Pflichtversicherung in } \\
\text { der Kranken- und Pensionsversicherung." }\end{array}$ \\
\hline KB & $\begin{array}{l}\text { Selbstversicherung bei ger- } \\
\text { ingfügiger Beschäftigung gem. } \\
\S 7 \text { a Abs. } 2 \text { Z } 1 \text { B-KUVG - } \\
\text { Angestellter }\end{array}$ & $\begin{array}{l}\text { "Zeiten einer Selbstversicherung als Angestellter bei geringfügiger Beschäftigung } \\
\text { gemäß } § 7 \text { a Abs. } 2 \text { Z } 1 \text { B-KUVG: Zeiten eines Krankengeld- bzw. Wochengeld- } \\
\text { bezuges, deren Anspruch aus dieser Selbstversicherung resultiert, sind unter der } \\
\text { gleichen "Dienstgeberkontonummer" mittels den Qualifikationen } 36 \text { und } 34 \mathrm{zu} \\
\text { melden. Diese Qualifikationsmeldung repräsentiert eine Pflichtversicherung in der } \\
\text { Kranken- und Pensionsversicherung." }\end{array}$ \\
\hline $\mathrm{KC}$ & $\begin{array}{l}\text { Selbstversicherung bei ger- } \\
\text { ingfügiger Beschäftigung gem. } \\
\S 7 \text { a Abs. } 2 \text { Z } 2 \text { B-KUVG - } \\
\text { Beamter/in bzw. Mandatar/in }\end{array}$ & $\begin{array}{l}\text { "Zeiten einer Selbstversicherung in der Krankenversicherung als Beamter/in } \\
\text { oder Mandatar/in bei geringfügiger Beschäftigung gemäß } \S 7 \mathrm{a} \text { Abs. } 2 \text { Z } 2 \text { B- } \\
\text { KUVG. Diese Qualifikationsmeldung repräsentiert eine Pflichtversicherung in der } \\
\text { Kranken- und Pensionsversicherung." }\end{array}$ \\
\hline L1 & $\begin{array}{l}\text { Beiträge (noch) nicht bezahlt ( } \\
\text { zu QUAL } 64 \text { ) }\end{array}$ & $\begin{array}{l}\text { "vorgeschriebenen Beiträge aber (noch) nicht bezahlt wurden den verschiedenen } \\
\text { Pflichtversicherungszeiten nach dem BSVG zuordnen zu können, wurden die Qual- } \\
\text { ifikationen L1 bis L7 geschaffen. Sie sind wie folgt zuzuordnen: L1 zu Pflichtver- } \\
\text { sicherungszeiten unter Qualifikation } 64 \text { L2 " " " } 96 \text { L3 " " " } 97 \text { L4 " " " } 20 \\
\text { L5 " " "99 L6" " " A8 L7" " " A9" }\end{array}$ \\
\hline L2 & $\begin{array}{l}\text { Beiträge (noch) nicht bezahlt ( } \\
\text { zu QUAL 96) }\end{array}$ & \\
\hline L3 & $\begin{array}{l}\text { Beiträge (noch) nicht bezahlt ( } \\
\text { zu QUAL 97) }\end{array}$ & \\
\hline L4 & $\begin{array}{l}\text { Beiträge (noch) nicht bezahlt ( } \\
\text { zu QUAL 20) }\end{array}$ & \\
\hline L5 & $\begin{array}{l}\text { Beiträge (noch) nicht bezahlt ( } \\
\text { zu QUAL 99) }\end{array}$ & \\
\hline L6 & $\begin{array}{l}\text { Beiträge (noch) nicht bezahlt ( } \\
\text { zu QUAL A8) }\end{array}$ & \\
\hline L8 & $\begin{array}{l}\text { Verjährung / Schuldenerlass } \\
\text { imRahmen des GSVG, FSVG }\end{array}$ & $\begin{array}{l}\text { "Alle Zeiten, in denen eine Pflichtversicherung nach dem GSVG bzw. FSVG } \\
\text { besteht, die Beiträge jedoch nicht mehr eingefordert werden dürfen bzw. verjährt } \\
\text { sind. Zeiträume unter der Qualifikation "L8" beziehen sich auf gespeicherte } \\
\text { Pflichtversicherungszeiten und werden parallel zu diesen gespeichert." }\end{array}$ \\
\hline L9 & $\begin{array}{l}\text { Nicht vorgeschriebene } \\
\text { Krankenversicherungsbeitrags- } \\
\text { grundlage }\end{array}$ & $\begin{array}{l}\text { "Alle Zeiten, für die bei der Sozialversicherungsanstalt der Bauern Krankenver- } \\
\text { sicherungsbeiträge wegen Vorliegens einer anderen Beschäftigung (Mehrfachver- } \\
\text { sicherung) nicht bzw. nur zum Teil vorgeschrieben werden, ist eine "Eintages- } \\
\text { meldung" (1.1.) unter oben genannter Qualifikation vorzusehen." }\end{array}$ \\
\hline
\end{tabular}

Continued on next page... 


\begin{tabular}{ll}
\multicolumn{1}{c}{.. table 1 continued } \\
\hline \hline qual & type \\
\hline M1 & $\begin{array}{l}\text { Unwirksame } \\
\text { (zu QUAL 64) }\end{array}$ \\
& \\
& \\
M2 & $\begin{array}{l}\text { Unwirksame Beitragszahlung } \\
\text { (zu QUAL 96) }\end{array}$ \\
M3 & $\begin{array}{l}\text { Unwirksame Beitragszahlung } \\
\text { (zu QUAL 97) }\end{array}$ \\
M4 & $\begin{array}{l}\text { Unwirksame Beitragszahlung } \\
\text { (zu QUAL 20) }\end{array}$ \\
M8 & $\begin{array}{l}\text { Hauptberuflich beschäftigte El- } \\
\text { tern im Rahmen des BSVG }\end{array}$ \\
M9 & $\begin{array}{l}\text { Beitragserstattung gem. } \\
\text { Abs. 5 ASVG }\end{array}$ \\
&
\end{tabular}

description

"Um Zeiträume, für die eine Pflichtversicherung nach dem BSVG besteht, die Beiträge aber verspätet und somit für die Anwartschaftsfeststellung in der Pensionsversicherung unwirksam entrichtet wurden, den verschiedenen Pflichtversicherungszeiten nach dem BSVG zuordnen zu können, wurden die Qualifikationen M1 bis M7 geschaffen. Sie sind wie folgt zuzuordnen: M1 zu Pflichtversicherungszeiten unter Qualifikation 64 M2 " " " 96 M3 " " " 97 M4 " " " 20 M5 " " " 99 M6 " " " A8 M7 " " " A9"

Bezug einer Versehrtenrente aufgrund von weniger als $50 \%$ Erwerbsunfähigkeitseinbuße Bezug einer Vollrente gem. § 205 Abs. 2 Z 1 ASVG bzw. § 103 Abs. 2 Z 1 B-KUVG Bezug einer Hinterbliebenenrente aus der gesetzlichen Unfallversicherung Weiterbildungsgeld nach dem ALVG $1977 \mathrm{ab}$ dem 45. LJ Sicherung gem. § 5d AMPFG - $\quad$ ASVG-Versicherungsträger (keine Beitragszahlung) (keine Beitragszahlung) BSVG-Versicherungsträger (keine Beitragszahlung) Versicherungsmonate)

NU Weiterbildungsgeld nach dem ALVG 1977 vor dem 45. LJ

O1 Beitragspflichtige Mitversicherung in der Krankenversicherung
"Alle Zeiten, die einer Tätigkeit als hauptberuflich beschäftigte Eltern gemäß den Bestimmungen des $\S 2$ Abs. 1 Ziffer 4 BSVG zugrunde liegen."

"Alle Zeiten, für die der Pensionsversicherungsträger Versicherten, die im Rahmen eines pensionsversicherungsfreien Dienstverhältnisses gegen Entfall der Bezüge beurlaubt sind und während des Karenzurlaubes eine die Pflichtversicherung in der Pensionsversicherung nach dem ASVG begründende Erwerbstätigkeit ausüben, die Beiträge gemäß den Bestimmungen des $\S 70$ Abs. 5 ASVG erstattet hat."

"Alle Zeiten, in denen ein bescheidmäßig zuerkannter Anspruch auf eine Versehrtenrente aus der gesetzlichen Unfallversicherung aufgrund einer Erwerbsunfähigkeitseinbuße von weniger als $50 \%$ besteht."

"Alle Zeiten, in denen ein bescheidmäßig zuerkannter Anspruch auf eine sogenannte Vollrente gemäß $\S 205$ Abs. 2 Z 1 ASVG bzw. $§ 103$ Abs. 2 Z 1 B-KUVG besteht."

"Alle Zeiten, in denen ein bescheidmäßig zuerkannter Anspruch auf eine Hinterbliebenenrente aus der gesetzlichen Unfallversicherung besteht."

"Alle Zeiten, während derer der Versicherte nach Vollendung des 45. Lebensjahres Weiterbildungsgeld nach dem Arbeitslosenversicherungsgesetz 1977 bezog."

"Personen, die krankenversicherungspflichtig erwerbstätig sind und nicht der Versicherungspflicht nach dem Arbeitslosenversicherungsgesetz 1977 unterliegen, sowie unselbständig Beschäftigte im Ausland können sich zur Zahlung eines Sicherungsbeitrages gem. $\S 5 \mathrm{~d}$ Arbeitsmarktpolitik-Finanzierungsgesetz (AMPFG) verpflichten. Für die ersten drei Jahre nach dem Ende der letzten Pflichtversicherung in der Arbeitslosenversicherung ist jedoch kein Sicherungsbeitrag zu entrichten. Dieser "beitragsfreie" Zeitraum ist zu dokumentieren."

"Personen, die krankenversicherungspflichtig erwerbstätig sind und nicht der Versicherungspflicht nach dem Arbeitslosenversicherungsgesetz 1977 unterliegen sowie unselbständig Beschäftigte im Ausland können sich zur Zahlung eines Sicherungsbeitrages gem. $\S 5 \mathrm{~d}$ Arbeitsmarktpolitik-Finanzierungsgesetz (AMPFG) verpflichten. Für die ersten drei Jahre nach dem Ende der letzten Pflichtversicherung in der Arbeitslosenversicherung ist jedoch kein Sicherungsbeitrag zu entrichten. Dieser "beitragsfreie" Zeitraum ist zu dokumentieren."

"Personen, die krankenversicherungspflichtig erwerbstätig sind und nicht der Versicherungspflicht nach dem Arbeitslosenversicherungsgesetz 1977 unterliegen sowie unselbständig Beschäftigte im Ausland können sich zur Zahlung eines Sicherungsbeitrages gem. $\S 5 \mathrm{~d}$ Arbeitsmarktpolitik-Finanzierungsgesetz (AMPFG) verpflichten. Für die ersten drei Jahre nach dem Ende der letzten Pflichtversicherung in der Arbeitslosenversicherung ist jedoch kein Sicherungsbeitrag zu entrichten. Dieser "beitragsfreie" Zeitraum ist zu dokumentieren."

"Zeiten eines Gleitpensionsbezuges gem. § 253c Abs.1 Z 1 lit.b ASVG, § 131b Abs. $1 \mathrm{Z} 1$ lit.b GSVG und $\S 122 \mathrm{~b}$ Abs. 1 Z 1 lit.b BSVG."

"Unter oben genannter Qualifikation sind jene Zeiten zu melden, während derer der Versicherte vor Vollendung des 45. Lebensjahres Weiterbildungsgeld nach dem Arbeitslosenversicherungsgesetz 1977 bezog (Freistellung bei gleichzeitiger Einstellung einer Ersatzkraft)."

"Alle Zeiten, für die ein Zusatzbeitrag gemäß § 51d ASVG bzw. § 20b B-KUVG zu leisten ist, sind unter oben genannter Qualifikation unter der Versicherungsnummer des Versicherten (nicht des Mitversicherten) zu melden."

Continued on next page... 


\begin{tabular}{|c|c|c|}
\hline qual & type & description \\
\hline $\mathrm{O} 2$ & $\begin{array}{l}\text { Beitragspflichtige Mitver- } \\
\text { sicherung in der Krankenver- } \\
\text { sicherung (GSVG/BSVG) }\end{array}$ & $\begin{array}{l}\text { "Alle Zeiten, für die ein Zusatzbeitrag gemäß } \S 27 \mathrm{c} \text { GSVG bzw. § } 24 \mathrm{c} \text { BSVG zu } \\
\text { leisten ist, sind unter oben genannter Qualifikation unter der Versicherungsnum- } \\
\text { mer des Versicherten (nicht des Mitversicherten) zu melden." }\end{array}$ \\
\hline $\mathrm{O} 3$ & $\begin{array}{l}\text { KV-Pflichtversicherung nach } \\
\text { dem ASVG - Auslandsbe- } \\
\text { treute mit ständigem Wohnsitz } \\
\text { in Österreich ('De-facto- } \\
\text { Versicherte') }\end{array}$ & $\begin{array}{l}\text { "Alle Zeiten von Auslandsbetreuten mit ständigem Wohnsitz in Österreich sind } \\
\text { unter oben genannter Qualifikation zu melden." }\end{array}$ \\
\hline $\mathrm{O} 4$ & $\begin{array}{l}\text { KV-Pflichtversicherung nach } \\
\text { dem ASVG - Asylwerber bzw. } \\
\text { Flüchtlinge }\end{array}$ & $\begin{array}{l}\text { "Alle Zeiten von Asylwerben bzw. Flüchtlingen (ASVG) sind unter oben genan- } \\
\text { nter Qualifikation zu melden." }\end{array}$ \\
\hline O5 & $\begin{array}{l}\text { KV-Pflichtversicherung nach } \\
\text { dem ASVG - Zugeteilte nach } \\
\text { dem OFG }\end{array}$ & $\begin{array}{l}\text { "Alle Zeiten von zugeteilten Personen nach dem Opferfürsorgegesetz (ohne Leis- } \\
\text { tungseinschränkung) sind unter oben genannter Qualifikation zu melden." }\end{array}$ \\
\hline O6 & $\begin{array}{l}\text { KV-Pflichtversicherung nach } \\
\text { dem ASVG - Kriegshin- } \\
\text { terbliebene }\end{array}$ & $\begin{array}{l}\text { "Alle Zeiten von Kriegshinterbliebenen, die eine Hinterbliebenenrente nach } \S 68 \\
\text { bzw. § } 69 \text { Kriegsopferversorgungsgesetz beziehen, sind unter oben genannter } \\
\text { Qualifikation zu melden." }\end{array}$ \\
\hline $\mathrm{O} 7$ & $\begin{array}{l}\text { Ruhen des KV-Anspruchs für } \\
\text { den Hauptversicherten }\end{array}$ & $\begin{array}{l}\text { "Um jene Fälle im Rahmen der KV-Anspruchsermittlung für die SV-Chipkarte ab- } \\
\text { decken zu können, in denen der Versicherte aufgrund eines Auslandsaufenthaltes } \\
\text { keinen KV-Anspruch bei einem österreichischen Krankenversicherungsträger hat } \\
\text { (bzw. in denen der KV-Anspruch ruht), seine in Österreich bleibenden } \\
\text { Angehörigen aber weiterhin KV-Anspruch haben, ist es erforderlich, diese Zeiten } \\
\text { mit einer eigenen Qualifikation zu melden. Des weiteren ist diese Qualifikation } \\
\text { auch für die Angehörigen der Fremdkassenbetreuten der Krankenfürsorgeanstalten } \\
\text { zu verwenden." }\end{array}$ \\
\hline O8 & Familienhospizkarenz & $\begin{array}{l}\text { "Familienhospiz und der daraus resultierende Kranken- und Pensionsver- } \\
\text { sicherungsschutz aufgrund einer Freistellung gegen Entfall des Entgeltes bzw. } \\
\text { eines Entgeltbezuges der unter der Geringfügigkeitsgrenze liegt (Geringfügig } \\
\text { Beschäftige). Ab 1.1.2005 ist der Tatbestand der Familienhospizkarenz unter den } \\
\text { beiden Qualifikationen } 09 \text { und OJ zu melden." }\end{array}$ \\
\hline O9 & $\begin{array}{l}\text { Familienhospizkarenz -nur PV- } \\
\text { Schutz }\end{array}$ & $\begin{array}{l}\text { "Familienhospiz und der daraus resultierende pensionsversicherungsrechtliche } \\
\text { Schutz au Grund einer Teilkarenzierung in Form einer Herabsetzung des Ent- } \\
\text { geltes unter dem Ausgleichszulagenrichtsatz. Ab 1.1.2005 ist der Tatbestand der } \\
\text { "klassischen" Familienhospizkarenz (KV- und PV-Schutz) jedenfalls mit den Qual- } \\
\text { ifikationen O9 und OJ zu melden" }\end{array}$ \\
\hline OJ & $\begin{array}{l}\text { Familienhospizkarenz }- \text { nur } \\
\text { KV-Schutz }\end{array}$ & $\begin{array}{l}\text { "Familienhospiz und der daraus resultierende ausschließliche krankenver- } \\
\text { sicherungsrechtliche Schutz aufgrund der Tatsache, dass kein oder lediglich ein } \\
\text { geringfügiges Einkommen vorliegt." }\end{array}$ \\
\hline OK & $\begin{array}{lrl}\text { Kein } & \text { KV-Anspruch } \\
\text { österreichischer EKVK }\end{array}$ & $\begin{array}{l}\text { "Unter dieser Qualifikation sind alle Zeiten von Auslandsbetreuten mit ständigem } \\
\text { Wohnsitz in Österreich zu melden, wenn aufgrund dieses Aufenthaltes keine } \\
\text { EKVK (Europäische Krankenversicherungskarte) ausgestellt werden darf. An- } \\
\text { merkung: Diese Qualifikation ist parallel zu einer bereits bestehenden Qualifika- } \\
\text { tion O3 (Auslandsbetreute mit ständigem Wohnsitz in Österreich - "De-facto- } \\
\text { Versicherte") zu melden." }\end{array}$ \\
\hline $\mathrm{P} 1$ & $\begin{array}{l}\text { Pflichtversicherung Werkver- } \\
\text { trag gem. } \& 4 \text { Abs. } 5 \text { ASVG - } \\
\text { Arbeiter }\end{array}$ & $\begin{array}{l}\text { "Alle Pflichtversicherungszeiten in der Pensionsversicherung der Arbeiter gemäß } \\
\S 4 \text { Abs. } 5 \text { ASVG (Werkverträge)." }\end{array}$ \\
\hline $\mathrm{P} 2$ & $\begin{array}{l}\text { Pflichtversicherung Werkver- } \\
\text { trag gem. } \$ 4 \text { Abs. } 5 \text { ASVG - } \\
\text { Angestellter }\end{array}$ & $\begin{array}{l}\text { "Alle Pflichtversicherungszeiten in der Pensionsversicherung der Angestellten } \\
\text { gemäß } § 4 \text { Abs. } 5 \text { ASVG (Werkverträge)." }\end{array}$ \\
\hline P3 & $\begin{array}{l}\text { Pflichtversicherung Werkver- } \\
\text { trag gem. } § 4 \text { Abs. } 4 \text { ASVG - } \\
\text { Arbeiter }\end{array}$ & $\begin{array}{l}\text { "Alle Pflichtversicherungszeiten in der Pensionsversicherung der Arbeiter gemäß } \\
\S 4 \text { Abs. } 4 \text { ASVG ("Freie Dienstverträge")." }\end{array}$ \\
\hline $\mathrm{P} 4$ & $\begin{array}{l}\text { Pflichtversicherung Werkver- } \\
\text { trag gem. } § 4 \text { Abs. } 4 \text { ASVG - } \\
\text { Angestellter }\end{array}$ & $\begin{array}{l}\text { "Alle Pflichtversicherungszeiten in der Pensionsversicherung der Angestellten } \\
\text { gemäß } § 4 \text { Abs. } 4 \text { ASVG ("Freie Dienstverträge")." }\end{array}$ \\
\hline P5 & $\begin{array}{l}\text { Anrechnungsbetrag gem. } \S 13 \\
\text { Bundesbezügegesetz }\end{array}$ & Zeiten eines Anrechnungsbetrages gemäß $\S 13$ Bundesbezügegesetz. \\
\hline P6 & $\begin{array}{l}\text { Überweisungsbetrag gem. } \S \\
49 \mathrm{~h} \text { Abs. } 3 \text { des Bezügegesetzes } \\
\text { / Anrechnungsbetrag gem. } \S 13 \\
\text { Bundesbezügegesetz }\end{array}$ & Zeiten eines Überweisungsbetrages gemäß $\S 49 \mathrm{~h}$ Abs. 3 des Bezügegesetzes. \\
\hline
\end{tabular}

Continued on next page... 


\begin{tabular}{|c|c|c|}
\hline qual & type & description \\
\hline P7 & $\begin{array}{l}\text { Freie Dienstverträge gem. } \S \\
4 \text { Abs. } 4 \text { ASVG - geringfügig } \\
\text { beschäftigt - Arbeiter }\end{array}$ & $\begin{array}{l}\text { "Alle Zeiten, in denen ein freier Dienstvertrag gem. § } 4 \text { Abs.4 ASVG vorliegt, } \\
\text { wenn der jeweilige Grenzwert für die Geringfügigkeit nicht überschritten wird. } \\
\text { Nur Unfallversicherung!" }\end{array}$ \\
\hline P8 & $\begin{array}{l}\text { Freie Dienstverträge gem. } \S \\
4 \text { Abs. } 4 \text { ASVG - geringfügig } \\
\text { beschäftigt - Angestellter }\end{array}$ & $\begin{array}{l}\text { "Alle Zeiten, in denen ein freier Dienstvertrag gem. } \S 4 \text { Abs.4 ASVG als } \\
\text { Angestellter vorliegt, wenn der jeweilige Grenzwert für die Geringfügigkeit nicht } \\
\text { überschritten wird. Nur Unfallversicherung!" }\end{array}$ \\
\hline P9 & Beiträge nicht bezahlt & $\begin{array}{l}\text { "Diese Qualifikation dient den Krankenversicherungsträgern zur Speicherung von } \\
\text { Pflichtversicherungszeiten, für die noch keine Beiträge wirksam entrichtet wur- } \\
\text { den, jedoch die Möglichkeit einer wirksamen Entrichtung besteht. (Zeitraum in- } \\
\text { nerhalb der 5-jährigen Verjährungsfrist laut } \S 68 \text { ASVG). Beispiel: Werden An- } \\
\text { meldungen nicht innerhalb von sechs Monaten präsentiert, ist das Präsenzdatum } \\
\text { als vorläufiger Beginn der Pflichtversicherung in Vormerkung zu nehmen. Die } \\
\text { Zeit vom tatsächlichen Beginn der Beschäftigung bis zum fiktiven Beginn der } \\
\text { Pflichtversicherung ist vorläufig unter dieser Qualifikation in Vormerkung zu brin- } \\
\text { gen. Werden die Beiträge innerhalb der im } \S 68 \text { ASVG vorgesehenen Fristen } \\
\text { bezahlt, erfolgt eine Änderung der Qualifikation von P9 auf } 10 \text { bzw. 14." }\end{array}$ \\
\hline Q1 & $\begin{array}{l}\text { Teilversicherung in der } \\
\text { Krankenversicherung gem. } \\
\S 43 \text { Abs. } 1 \text { bzw. Abs. } 2 \text { KGG }\end{array}$ & $\begin{array}{l}\text { "Alle Zeiten, in denen eine Teilversicherung in der Krankenversicherung gemäß } \\
\text { den Bestimmungen des } \S 43 \text { Abs. } 2 \text { KGG vorliegt. Nur für VSTR 07: Alle Zeiten, in } \\
\text { denen eine Teilversicherung in der Krankenversicherung gemäß den Bestimmungen } \\
\text { des } § 43 \text { Abs. } 1 \text { bzw. Abs. } 2 \text { vorliegt." }\end{array}$ \\
\hline Q2 & $\begin{array}{l}\text { Pflichtversicherung in der } \\
\text { Krankenversicherung nach dem } \\
\text { ASVG (auf DG-Kontonummer } \\
\text { bezogen) }\end{array}$ & $\begin{array}{l}\text { "Zeiten einer Pflicht(Teil-)versicherung in der Krankenversicherung nach dem } \\
\text { ASVG. Unter dieser Qualifikation wurden die Personengruppen des } \S 4 \text { Abs. } 3 \\
\text { und } \S 8 \text { Abs.1 Z } 4 \mathrm{~b} \text { und } \mathrm{Z} 4 \mathrm{c} \text { ASVG gemeldet. Ab } 1 \text {. Jänner } 2001 \text { sind unter } \\
\text { dieser Qualifikation sämtliche Teilversicherungen in der Krankenversicherung nach } \\
\text { dem ASVG zu melden. Diese sind insbesondere Zeiten gemäß den Bestimmungen } \\
\text { der oben angeführten Paragrafen sowie des } \S 7 \mathrm{Z} .1, \S 8 \text { Abs. } 1 \mathrm{Z} .1 \text { lit. b } \\
\text { bis c und e } \S 9 \text { ASVG, Leistungszugeteilte auf Grund der Bestimmungen des } \\
\text { HVG und Bezieher von Arbeitslosengeld (Notstandshilfe, Pensionsvorschuss etc.). } \\
\text { Des Weiteren werden unter dieser Qualifikation Zeiten einer Dienstfreistellung } \\
\text { für Vertragsbedienstete in Anwendung des } \S 160 \text { BDG gegen Entfall der Bezüge } \\
\text { gemeldet. Weiters sind unter dieser Qualifikation die Fremdkassenbetreuten der } \\
\text { Krankenfürsorgeanstalten einzuspeichern." }\end{array}$ \\
\hline Q3 & $\begin{array}{l}\text { Pflichtversicherung in der } \\
\text { Krankenversicherung nach dem } \\
\text { ASVG }\end{array}$ & $\begin{array}{l}\text { "Alle Zeiten für die unter der Qualifikation Q2 angeführten Personengruppen, bei } \\
\text { denen aus versicherungsträgerspezifischen Gründen keine Dienstgeberkontonum- } \\
\text { mer gemeldet werden kann." }\end{array}$ \\
\hline Q4 & $\begin{array}{l}\text { Kein Leistungsanspruch in der } \\
\text { Krankenversicherung }\end{array}$ & $\begin{array}{l}\text { "Diese Qualifikation soll zusätzlich zu den "Vollversicherungsqualifikationen" } \\
\text { (z.B. Qualifikation } 10 \text { oder 14) gemeldet werden und zeigt an, dass diese Qual- } \\
\text { ifikation keine Krankenversicherungspflicht begründet, sondern nur eine Pen- } \\
\text { sionsversicherungspflicht (Negativmeldung). Diese Meldung ist für Personen, die } \\
\text { gemäß den Bestimmungen des } \S 7 \mathrm{Z} .2 \mathrm{ASVG} \text { versichert sind, sowie für fallweise } \\
\text { Beschäftigte, sofern sie die "Wartezeitbestimmungen" des } \S 468 \text { ASVG noch nicht } \\
\text { erfüllt haben, erforderlich." }\end{array}$ \\
\hline Q5 & $\begin{array}{l}\text { Selbstversicherung in der } \\
\text { Krankenversicherung } \text { gem. } \\
\S 16 \quad \text { ASVG- 'Wartezeit'- } \\
\text { Übergangslösung }\end{array}$ & $\begin{array}{l}\text { "Jene Versicherungsträger, welche die neuen Qualifikationen für die Selbstver- } \\
\text { sicherung in der Krankenversicherung gemäß } \S 16 \text { ASVG noch nicht einsetzen } \\
\text { (Qualifikationen I1, I2 und I3), können zur Dokumentation von Zeiten, für die } \\
\text { gemäß den Bestimmungen des } \S 124 \text { ASVG kein Leistungsanspruch besteht, die } \\
\text { oben angeführte Qualifikation verwenden." }\end{array}$ \\
\hline Q6 & $\begin{array}{l}\text { Sicherungsbeitrag gem. } \S 5 \mathrm{~d} \\
\text { AMPFG - Beitragszahlung bei } \\
\text { ASVG-Versicherungsträger }\end{array}$ & $\begin{array}{l}\text { "Personen, die krankenversicherungspflichtig erwerbstätig sind und nicht der } \\
\text { Versicherungspflicht nach dem Arbeitslosenversicherungsgesetz } 1977 \text { unterliegen } \\
\text { sowie unselbständig Beschäftigte im Ausland können sich zur Zahlung eines } \\
\text { Sicherungsbeitrages gem. } \$ 5 \text { d Arbeitsmarktpolitik- Finanzierungsgesetz } \\
\text { (AMPFG) verpflichten." }\end{array}$ \\
\hline Q7 & $\begin{array}{l}\text { Sicherungsbeitrag gem. } \S 5 \mathrm{~d} \\
\text { AMPFG - Beitragszahlung bei } \\
\text { GSVG-VSTR }\end{array}$ & $\begin{array}{l}\text { "Personen, die krankenversicherungspflichtig erwerbstätig sind und nicht der } \\
\text { Versicherungspflicht nach dem Arbeitslosenversicherungsgesetz } 1977 \text { unterliegen } \\
\text { sowie unselbständig Beschäftigte im Ausland können sich zur Zahlung eines } \\
\text { Sicherungsbeitrages gem. } \S 5 \mathrm{~d} \text { Arbeitsmarktpolitik- Finanzierungsgesetz } \\
\text { (AMPFG)verpflichten." }\end{array}$ \\
\hline Q8 & $\begin{array}{l}\text { Sicherungsbeitrag gem. } \S 5 \mathrm{~d} \\
\text { AMPFG - Beitragszahlung bei } \\
\text { BSVG-VSTR }\end{array}$ & $\begin{array}{l}\text { "Personen, die krankenversicherungspflichtig erwerbstätig sind und nicht der } \\
\text { Versicherungspflicht nach dem Arbeitslosenversicherungsgesetz } 1977 \text { unterliegen } \\
\text { sowie unselbständig Beschäftigte im Ausland können sich zur Zahlung eines } \\
\text { Sicherungsbeitrages gem. } § 5 \mathrm{~d} \text { Arbeitsmarktpolitik- Finanzierungsgesetz } \\
\text { verpflichten." }\end{array}$ \\
\hline Q9 & Erntehelfer & $\begin{array}{l}\text { "Alle Zeiten, die einer Tätigkeit als Erntehelfer gemäß den Bestimmungen des } \S \\
7 \text { Ziffer } 1 \text { lit. f ASVG zu Grunde liegen." }\end{array}$ \\
\hline
\end{tabular}

Continued on next page... 


\begin{tabular}{lll}
\hline \hline qual & type & description \\
\hline Y1 & PV-Pflichtversicherung nach & "Alle Pflichtversicherungszeiten als Arbeiter in der knappschaftlichen Pen- \\
dem ASVG (Arbeiter in der & sionsversicherung mit Ausnahme der Sonderfälle mit Qualifikation 11 - 12, B1, \\
knappschaftl. PV) & B2, B2, C1, D1 sowie G1 - G6. Zeiträume bis 31.12.2004 sind unter der Quali- \\
& fikation 10 vom Versicherungsträger 04 zu melden"
\end{tabular}

Bezug von vorzeitiger Al- "Alle Zeiten, in denen ein bescheidmäßig zuerkannter Anspruch auf eine vorzeitterspension bei langer Ver- ige Alterspension bei langer Versicherungsdauer aus der knappschaftlichen Pensicherungsdauer aus der sionsversicherung besteht. Zeiträume bis 31.12.2004 sind unter der Qualifikation knappschaftl. PV D5 vom Versicherungsträger 04 zu melden."

Bezug einer Knappschaftsalterspension der knappschaftl. PV)

"Alle Zeiten, in denen ein bescheidmäßig zuerkannter Anspruch auf eine vorzeitige Alterspension bei langer Versicherungsdauer aus der knappschaftlichen Pensionsversicherung besteht. Zeiträume bis 31.12.2004 sind unter der Qualifikation D5 vom Versicherungsträger 04 zu melden."

"Alle Pflichtversicherungszeiten als Lehrling in der knappschaftlichen Pensionsversicherung - Arbeiter, mit Ausnahme der Sonderfälle mit Qualifikation B2. Zeiträume bis 31.12.2004 sind unter der B1 vom Versicherungsträger $04 \mathrm{zu}$ melden." knappschaftl. PV) PV)

"Alle Zeiten, in denen ein bescheidmäßig zuerkannter Anspruch auf eine Gleitpension der knappschaftlichen Pensionsversicherung besteht. Ab 1.1.1998 sind unter dieser Qualifikation nur Gleitpensionen gemäß § 253c Abs. 1 Z1 lit. a ASVG, § 131b Abs. $1 \mathrm{Z} 1$ lit. a GSVG und $\S 122 \mathrm{~b}$ Abs. $1 \mathrm{Z} 1$ lit. a BSVG zu speichern (siehe auch Kapitel D.6.N9.). Zeiträume bis 31.12.2004 sind unter der Qualifikation D9 vom Versicherungsträger 04 zu melden."

"Alle Pflichtversicherungszeiten als Angestelltenlehrling in der knappschaftlichen Pensionsversicherung. Zeiträume bis 31.12.2004 sind unter der Qualifikation B4 vom Versicherungsträger 04 zu melden."

"Alle Pflichtversicherungszeiten als Arbeiter in der knappschaftlichen Pensionsversicherung nach dem ASVG, bei Vorliegen einer Beschäftigung gem. dem NSchG mit Ausnahme der Sonderfälle mit Qualifikation G2, G3. Zeiträume bis 31.12.2004 sind unter der Qualifikation G1 vom Versicherungsträger $04 \mathrm{zu}$ melden."

"Alle Pflichtversicherungszeiten als Angestellter in der knappschaftlichen Pensionsversicherung mit Ausnahme der Sonderfälle mit Qualifikation G5, G6. Zeiträume bis 31.12.2004 sind unter der Qualifikation G4 vom Versicherungsträger 04 zu melden."

"Alle Pflichtversicherungszeiten als Angestellter in der knappschaftlichen Pensionsversicherung gemäß $\S 4$ Abs. 4 ASVG ("Freie Dienstverträge"). Zeiträume bis 31.12.2004 sind unter der Qualifikation P4 vom Versicherungsträger $04 \mathrm{zu}$ melden."

ZM Bezug einer vorzeitigen Alterspension wegen geminderter Erwerbsfähigkeit aus der knappschaftl. PV

"Alle Zeiten, in denen ein bescheidmäßig zuerkannter Anspruch auf eine vorzeitige Alterspension wegen geminderter Erwerbsfähigkeit aus der knappschaftlichen Pensionsversicherung besteht. Zeiträume bis 31.12.2004 sind unter der Qualifikation D7 vom Versicherungsträger 04 zu melden." 
Panel A: Employment

Panel A.1: Regular Employment

101112141516 A5 A6 B1 B2 B4 B8 B9 C1 C4 C6 C7 D1 E4 G1 G2 G3 G4 G5 G6 G7 P1 P2 P3 P4 Y1 Y4 Z1 Z4 ZC ZD ZJ

Panel A.2: Self Employed incl. Farmers and Relatives of Farmers primarily occupied on the farm

1718192064969799 A8 A9 F1 F2 F3 F4 M8

Panel A.3: Employment as Civil Servant

BB J1 J8 J9 JA JN

Panel A.4: Special Forms of Employment

60 A2 G8 G9 GD GT P7 P8 Q9

Panel B: Sickness

$36373 \mathrm{G} 86$

Panel C: Unemployment

$38395681 \mathrm{C} 5$

Panel D: Retirement

Panel D.1: Old-age Pensions

09 D5 D6 J5 Y6 Y8 Y9

Panel D.2: Disability Pensions and the like

0708 D7 Y7 ZM

Panel D.3: Special Forms of Retirement

054048 D8 D9 N9 Z3 Y5

Panel E: Special Interests

PanelE.1: Parental Leave

$34353 \mathrm{E} 4151 \mathrm{~J} 2$

Panel E.2: Additional Spells related to Maternity

4987 E3 EX I5 I7

Panel E.3: Benefit Payments not linked to Current Employment Status 066365 D2 D3 D4 J6 J7 N3

Panel E.4: Accident Benefits and Reintegration Measures after Accidents

$426668 \mathrm{~N} 1 \mathrm{~N} 2$

Panel E.5: Reintegration Support for Unemployed

A1 A3 A4 N4

Panel E.6: Education

$303132 \mathrm{H} 1 \mathrm{H} 2 \mathrm{H} 3 \mathrm{H} 4 \mathrm{HB}$

Panel E.7: Military and Civilian Service

33 3A 3B 53

Panel E.8: Voluntary Contribution in Pension Insurance

2526272829 E1 E2 E5 E6 E7 E8 E9 F5 F6 F7 F8 F9 K2 K3 K4 K5 K6 K7

Panel F: Other

$010213212223242 \mathrm{~A} 3 \mathrm{~J} 3 \mathrm{~T} 43444546474 \mathrm{~A} 4 \mathrm{~B} 4 \mathrm{C} 4 \mathrm{D} 4 \mathrm{E} \quad 4 \mathrm{~F} 4 \mathrm{G} 4 \mathrm{H} 4 \mathrm{I} 4 \mathrm{~J} 4 \mathrm{~K} 4 \mathrm{~L} 4 \mathrm{M} 4 \mathrm{~N} 4 \mathrm{P} 4 \mathrm{R} 4 \mathrm{Z} 5052$ 545557616269707879808385 A7 BD BE BK C2 C3 C8 C9 CX I1 I2 I3 I4 I8 I9 IA IB J3 JL JM K1 K9 KA KB KC L1 L2 L3 L4 L5 L6 L8 L9 M1 M2 M3 M4 M9 N6 N7 N8 NU O1 O2 O3 O4 O5 O6 O7 O8 O9 OJ OK P5 P6 P9 Q1 Q2 Q3 Q4 Q5 Q6 Q7 Q8 
Table 3: Dates at which qualifications are first introduced

\begin{tabular}{|c|c|c|c|}
\hline \multicolumn{2}{|c|}{ sorted by qual } & \multicolumn{2}{|c|}{ sorted by date } \\
\hline qual & date & qual & date \\
\hline 01 & $12 / 31 / 1971$ & 49 & $1 / 1 / 1971$ \\
\hline 02 & $4 / 11 / 1998$ & 01 & $12 / 31 / 1971$ \\
\hline 05 & $7 / 1 / 1981$ & 06 & $12 / 31 / 1971$ \\
\hline 06 & $12 / 31 / 1971$ & 07 & $12 / 31 / 1971$ \\
\hline 07 & $12 / 31 / 1971$ & 08 & $12 / 31 / 1971$ \\
\hline 08 & $12 / 31 / 1971$ & 09 & $12 / 31 / 1971$ \\
\hline 09 & $12 / 31 / 1971$ & 10 & $12 / 31 / 1971$ \\
\hline 10 & $12 / 31 / 1971$ & 11 & $12 / 31 / 1971$ \\
\hline 11 & $12 / 31 / 1971$ & 12 & $12 / 31 / 1971$ \\
\hline 12 & $12 / 31 / 1971$ & 14 & $12 / 31 / 1971$ \\
\hline 13 & $1 / 1 / 2000$ & 15 & $12 / 31 / 1971$ \\
\hline 14 & $12 / 31 / 1971$ & 16 & $12 / 31 / 1971$ \\
\hline 15 & $12 / 31 / 1971$ & 18 & $12 / 31 / 1971$ \\
\hline 16 & $12 / 31 / 1971$ & 19 & $12 / 31 / 1971$ \\
\hline 17 & $1 / 1 / 1972$ & 20 & $12 / 31 / 1971$ \\
\hline 18 & $12 / 31 / 1971$ & 21 & $12 / 31 / 1971$ \\
\hline 19 & $12 / 31 / 1971$ & 22 & $12 / 31 / 1971$ \\
\hline 20 & $12 / 31 / 1971$ & 23 & $12 / 31 / 1971$ \\
\hline 21 & $12 / 31 / 1971$ & 24 & $12 / 31 / 1971$ \\
\hline 22 & $12 / 31 / 1971$ & 25 & $12 / 31 / 1971$ \\
\hline 23 & $12 / 31 / 1971$ & 26 & $12 / 31 / 1971$ \\
\hline 24 & $12 / 31 / 1971$ & 27 & $12 / 31 / 1971$ \\
\hline 25 & $12 / 31 / 1971$ & 28 & $12 / 31 / 1971$ \\
\hline 26 & $12 / 31 / 1971$ & 29 & $12 / 31 / 1971$ \\
\hline 27 & $12 / 31 / 1971$ & 30 & $12 / 31 / 1971$ \\
\hline 28 & $12 / 31 / 1971$ & 31 & $12 / 31 / 1971$ \\
\hline 29 & $12 / 31 / 1971$ & 32 & $12 / 31 / 1971$ \\
\hline $2 \mathrm{~A}$ & $1 / 1 / 1993$ & 33 & $12 / 31 / 1971$ \\
\hline 30 & $12 / 31 / 1971$ & 34 & $12 / 31 / 1971$ \\
\hline 31 & $12 / 31 / 1971$ & 35 & $12 / 31 / 1971$ \\
\hline 32 & $12 / 31 / 1971$ & 36 & $12 / 31 / 1971$ \\
\hline 33 & $12 / 31 / 1971$ & 37 & $12 / 31 / 1971$ \\
\hline 34 & $12 / 31 / 1971$ & 38 & $12 / 31 / 1971$ \\
\hline 35 & $12 / 31 / 1971$ & 39 & $12 / 31 / 1971$ \\
\hline 36 & $12 / 31 / 1971$ & 41 & $12 / 31 / 1971$ \\
\hline 37 & $12 / 31 / 1971$ & 42 & $12 / 31 / 1971$ \\
\hline 38 & $12 / 31 / 1971$ & 47 & $12 / 31 / 1971$ \\
\hline 39 & $12 / 31 / 1971$ & 50 & $12 / 31 / 1971$ \\
\hline $3 \mathrm{~A}$ & $1 / 1 / 2002$ & 52 & $12 / 31 / 1971$ \\
\hline 3B & $1 / 1 / 2005$ & 54 & $12 / 31 / 1971$ \\
\hline $3 \mathrm{E}$ & $1 / 1 / 2005$ & 55 & $12 / 31 / 1971$ \\
\hline $3 \mathrm{G}$ & $1 / 1 / 2005$ & 57 & $12 / 31 / 1971$ \\
\hline $3 \mathrm{~J}$ & $1 / 1 / 2005$ & 62 & $12 / 31 / 1971$ \\
\hline $3 \mathrm{~T}$ & $1 / 1 / 2003$ & 70 & $12 / 31 / 1971$ \\
\hline 40 & $6 / 1 / 1978$ & 78 & $12 / 31 / 1971$ \\
\hline 41 & $12 / 31 / 1971$ & 79 & $12 / 31 / 1971$ \\
\hline 42 & $12 / 31 / 1971$ & 80 & $12 / 31 / 1971$ \\
\hline 43 & $1 / 1 / 1988$ & 85 & $12 / 31 / 1971$ \\
\hline 44 & $6 / 10 / 1972$ & 87 & $12 / 31 / 1971$ \\
\hline 45 & $8 / 19 / 1980$ & $\mathrm{~A} 1$ & $12 / 31 / 1971$ \\
\hline 46 & $6 / 26 / 1973$ & $\mathrm{~A} 4$ & $12 / 31 / 1971$ \\
\hline 47 & $12 / 31 / 1971$ & $\mathrm{~A} 7$ & $12 / 31 / 1971$ \\
\hline 48 & $3 / 1 / 1975$ & B1 & $12 / 31 / 1971$ \\
\hline 49 & $1 / 1 / 1971$ & B2 & $12 / 31 / 1971$ \\
\hline $4 \mathrm{~A}$ & $1 / 1 / 2000$ & $\mathrm{~B} 4$ & $12 / 31 / 1971$ \\
\hline $4 \mathrm{~B}$ & $1 / 1 / 2000$ & $\mathrm{C} 1$ & $12 / 31 / 1971$ \\
\hline $4 \mathrm{C}$ & $1 / 1 / 2000$ & $\mathrm{C} 4$ & $12 / 31 / 1971$ \\
\hline $4 \mathrm{D}$ & $1 / 1 / 2000$ & D1 & $12 / 31 / 1971$ \\
\hline $4 \mathrm{E}$ & $1 / 1 / 2000$ & E3 & $12 / 31 / 1971$ \\
\hline $4 \mathrm{~F}$ & $1 / 1 / 2000$ & E5 & $12 / 31 / 1971$ \\
\hline $4 \mathrm{G}$ & $1 / 1 / 1994$ & G1 & $12 / 31 / 1971$ \\
\hline $4 \mathrm{H}$ & $1 / 1 / 1994$ & G2 & $12 / 31 / 1971$ \\
\hline
\end{tabular}

Continued on next page... 


\begin{tabular}{|c|c|c|c|}
\hline \multicolumn{2}{|c|}{ sorted by qual } & \multicolumn{2}{|c|}{ sorted by date } \\
\hline qual & date & qual & date \\
\hline $4 \mathrm{I}$ & $1 / 1 / 2000$ & G4 & $12 / 31 / 1971$ \\
\hline $4 \mathrm{~J}$ & $1 / 1 / 2000$ & $\mathrm{H} 1$ & $12 / 31 / 1971$ \\
\hline $4 \mathrm{~K}$ & $1 / 1 / 2000$ & $\mathrm{H} 2$ & $12 / 31 / 1971$ \\
\hline $4 \mathrm{~L}$ & $1 / 1 / 2006$ & H3 & $12 / 31 / 1971$ \\
\hline $4 \mathrm{M}$ & $1 / 1 / 2000$ & $\mathrm{JM}$ & $12 / 31 / 1971$ \\
\hline $4 \mathrm{~N}$ & $1 / 1 / 2000$ & M4 & $12 / 31 / 1971$ \\
\hline $4 \mathrm{P}$ & $1 / 1 / 2000$ & 17 & $1 / 1 / 1972$ \\
\hline $4 \mathrm{R}$ & $4 / 1 / 2001$ & 60 & $1 / 1 / 1972$ \\
\hline $4 \mathrm{Z}$ & $1 / 1 / 1980$ & F5 & $1 / 1 / 1972$ \\
\hline 50 & $12 / 31 / 1971$ & G3 & $1 / 1 / 1972$ \\
\hline 51 & $1 / 1 / 1990$ & L8 & $1 / 1 / 1972$ \\
\hline 52 & $12 / 31 / 1971$ & 86 & $5 / 16 / 1972$ \\
\hline 53 & $4 / 1 / 1975$ & $\mathrm{H} 4$ & $6 / 1 / 1972$ \\
\hline 54 & $12 / 31 / 1971$ & 44 & $6 / 10 / 1972$ \\
\hline 55 & $12 / 31 / 1971$ & $\mathrm{JN}$ & $1 / 1 / 1973$ \\
\hline 56 & $1 / 1 / 1988$ & 83 & $5 / 8 / 1973$ \\
\hline 57 & $12 / 31 / 1971$ & 46 & $6 / 26 / 1973$ \\
\hline 60 & $1 / 1 / 1972$ & G5 & $9 / 1 / 1974$ \\
\hline 61 & $1 / 1 / 1988$ & 48 & $3 / 1 / 1975$ \\
\hline 62 & $12 / 31 / 1971$ & 53 & $4 / 1 / 1975$ \\
\hline 63 & $1 / 1 / 1976$ & 63 & $1 / 1 / 1976$ \\
\hline 64 & $1 / 1 / 1992$ & 65 & $1 / 1 / 1976$ \\
\hline 65 & $1 / 1 / 1976$ & 66 & $1 / 1 / 1977$ \\
\hline 66 & $1 / 1 / 1977$ & 68 & $1 / 1 / 1977$ \\
\hline 68 & $1 / 1 / 1977$ & 40 & $6 / 1 / 1978$ \\
\hline 69 & $9 / 1 / 1981$ & $\mathrm{E} 4$ & $1 / 1 / 1979$ \\
\hline 70 & $12 / 31 / 1971$ & E6 & $1 / 1 / 1979$ \\
\hline 78 & $12 / 31 / 1971$ & E7 & $1 / 1 / 1979$ \\
\hline 79 & $12 / 31 / 1971$ & E8 & $1 / 1 / 1979$ \\
\hline 80 & $12 / 31 / 1971$ & E9 & $1 / 1 / 1979$ \\
\hline 81 & $1 / 1 / 1993$ & $\mathrm{~F} 1$ & $1 / 1 / 1979$ \\
\hline 83 & $5 / 8 / 1973$ & F2 & $1 / 1 / 1979$ \\
\hline 85 & $12 / 31 / 1971$ & $4 \mathrm{Z}$ & $1 / 1 / 1980$ \\
\hline 86 & $5 / 16 / 1972$ & $\mathrm{C} 9$ & $1 / 1 / 1980$ \\
\hline 87 & $12 / 31 / 1971$ & 45 & $8 / 19 / 1980$ \\
\hline 96 & $1 / 1 / 1992$ & $\mathrm{~L} 4$ & $12 / 1 / 1980$ \\
\hline 97 & $1 / 1 / 1992$ & P9 & $1 / 1 / 1981$ \\
\hline 99 & $4 / 1 / 1988$ & 5 & $7 / 1 / 1981$ \\
\hline $\mathrm{A} 1$ & $12 / 31 / 1971$ & 69 & $9 / 1 / 1981$ \\
\hline A2 & $1 / 1 / 1995$ & P6 & $1 / 1 / 1984$ \\
\hline A3 & $8 / 14 / 1997$ & G6 & $5 / 5 / 1986$ \\
\hline A4 & $12 / 31 / 1971$ & 43 & $1 / 1 / 1988$ \\
\hline A5 & $7 / 1 / 1994$ & 56 & $1 / 1 / 1988$ \\
\hline A 6 & $7 / 1 / 1994$ & 61 & $1 / 1 / 1988$ \\
\hline A 7 & $12 / 31 / 1971$ & E1 & $1 / 1 / 1988$ \\
\hline A 8 & $7 / 1 / 1993$ & $\mathrm{~J} 1$ & $1 / 1 / 1988$ \\
\hline A9 & $7 / 1 / 1993$ & $\mathrm{~J} 2$ & $1 / 1 / 1988$ \\
\hline B1 & $12 / 31 / 1971$ & J5 & $1 / 1 / 1988$ \\
\hline B2 & $12 / 31 / 1971$ & J6 & $1 / 1 / 1988$ \\
\hline B4 & $12 / 31 / 1971$ & J7 & $1 / 1 / 1988$ \\
\hline B8 & $1 / 1 / 1998$ & 99 & $4 / 1 / 1988$ \\
\hline B9 & $1 / 1 / 1998$ & HB & $4 / 21 / 1988$ \\
\hline $\mathrm{BB}$ & $1 / 1 / 2006$ & 51 & $1 / 1 / 1990$ \\
\hline $\mathrm{BD}$ & $2 / 1 / 2006$ & 64 & $1 / 1 / 1992$ \\
\hline $\mathrm{BE}$ & $1 / 5 / 2006$ & 96 & $1 / 1 / 1992$ \\
\hline BK & $2 / 1 / 2006$ & 97 & $1 / 1 / 1992$ \\
\hline $\mathrm{C} 1$ & $12 / 31 / 1971$ & $\mathrm{E} 2$ & $1 / 1 / 1992$ \\
\hline $\mathrm{C} 2$ & $1 / 1 / 1997$ & M1 & $1 / 1 / 1992$ \\
\hline $\mathrm{C} 3$ & $1 / 1 / 1997$ & F8 & $4 / 1 / 1992$ \\
\hline $\mathrm{C} 4$ & $12 / 31 / 1971$ & L1 & $5 / 1 / 1992$ \\
\hline C5 & $1 / 1 / 1996$ & M2 & $10 / 1 / 1992$ \\
\hline $\mathrm{C} 6$ & $7 / 1 / 1993$ & F7 & $12 / 1 / 1992$ \\
\hline $\mathrm{C} 7$ & $7 / 1 / 1993$ & $2 \mathrm{~A}$ & $1 / 1 / 1993$ \\
\hline
\end{tabular}

Continued on next page... 


\begin{tabular}{|c|c|c|c|}
\hline \multicolumn{2}{|c|}{ sorted by qual } & \multicolumn{2}{|c|}{ sorted by date } \\
\hline qual & date & qual & date \\
\hline $\mathrm{C} 8$ & $1 / 1 / 1993$ & 81 & $1 / 1 / 1993$ \\
\hline C9 & $1 / 1 / 1980$ & $\mathrm{C} 8$ & $1 / 1 / 1993$ \\
\hline $\mathrm{CX}$ & $1 / 1 / 2005$ & F9 & $1 / 1 / 1993$ \\
\hline D1 & $12 / 31 / 1971$ & $\mathrm{~L} 2$ & $1 / 1 / 1993$ \\
\hline D2 & $5 / 1 / 1996$ & D8 & $2 / 1 / 1993$ \\
\hline D3 & $5 / 1 / 1996$ & M3 & $3 / 1 / 1993$ \\
\hline $\mathrm{D} 4$ & $3 / 10 / 1997$ & $\mathrm{~A} 8$ & $7 / 1 / 1993$ \\
\hline D5 & $7 / 1 / 1993$ & A9 & $7 / 1 / 1993$ \\
\hline D6 & $7 / 1 / 1993$ & $\mathrm{C} 6$ & $7 / 1 / 1993$ \\
\hline D7 & $7 / 1 / 1993$ & $\mathrm{C} 7$ & $7 / 1 / 1993$ \\
\hline D8 & $2 / 1 / 1993$ & D5 & $7 / 1 / 1993$ \\
\hline D9 & $7 / 1 / 1993$ & D6 & $7 / 1 / 1993$ \\
\hline $\mathrm{E} 1$ & $1 / 1 / 1988$ & D7 & $7 / 1 / 1993$ \\
\hline $\mathrm{E} 2$ & $1 / 1 / 1992$ & D9 & $7 / 1 / 1993$ \\
\hline E3 & $12 / 31 / 1971$ & $4 \mathrm{G}$ & $1 / 1 / 1994$ \\
\hline $\mathrm{E} 4$ & $1 / 1 / 1979$ & $4 \mathrm{H}$ & $1 / 1 / 1994$ \\
\hline E5 & $12 / 31 / 1971$ & G8 & $1 / 1 / 1994$ \\
\hline E6 & $1 / 1 / 1979$ & G9 & $1 / 1 / 1994$ \\
\hline $\mathrm{E} 7$ & $1 / 1 / 1979$ & I1 & $1 / 1 / 1994$ \\
\hline $\mathrm{E} 8$ & $1 / 1 / 1979$ & I2 & $1 / 1 / 1994$ \\
\hline E9 & $1 / 1 / 1979$ & I3 & $1 / 1 / 1994$ \\
\hline $\mathrm{EX}$ & $8 / 1 / 2000$ & K1 & $1 / 1 / 1994$ \\
\hline $\mathrm{F} 1$ & $1 / 1 / 1979$ & K9 & $1 / 1 / 1994$ \\
\hline $\mathrm{F} 2$ & $1 / 1 / 1979$ & Q5 & $1 / 1 / 1994$ \\
\hline F3 & $1 / 1 / 1998$ & A5 & $7 / 1 / 1994$ \\
\hline $\mathrm{F} 4$ & $1 / 1 / 1998$ & $\mathrm{~A} 6$ & $7 / 1 / 1994$ \\
\hline F5 & $1 / 1 / 1972$ & $\mathrm{~N} 2$ & $7 / 1 / 1994$ \\
\hline F6 & $4 / 1 / 1995$ & A2 & $1 / 1 / 1995$ \\
\hline F7 & $12 / 1 / 1992$ & M9 & $1 / 1 / 1995$ \\
\hline F8 & $4 / 1 / 1992$ & F6 & $4 / 1 / 1995$ \\
\hline F9 & $1 / 1 / 1993$ & C5 & $1 / 1 / 1996$ \\
\hline G1 & $12 / 31 / 1971$ & N1 & $1 / 1 / 1996$ \\
\hline G2 & $12 / 31 / 1971$ & N3 & $1 / 1 / 1996$ \\
\hline G3 & $1 / 1 / 1972$ & $\mathrm{JL}$ & $4 / 1 / 1996$ \\
\hline G4 & $12 / 31 / 1971$ & D2 & $5 / 1 / 1996$ \\
\hline G5 & $9 / 1 / 1974$ & D3 & $5 / 1 / 1996$ \\
\hline G6 & $5 / 5 / 1986$ & N6 & $5 / 1 / 1996$ \\
\hline G7 & $1 / 1 / 1998$ & N7 & $5 / 1 / 1996$ \\
\hline G8 & $1 / 1 / 1994$ & Q6 & $5 / 1 / 1996$ \\
\hline G9 & $1 / 1 / 1994$ & Q7 & $5 / 1 / 1996$ \\
\hline GD & $1 / 1 / 2006$ & Q8 & $5 / 1 / 1996$ \\
\hline GT & $1 / 1 / 2006$ & $\mathrm{P} 1$ & $7 / 1 / 1996$ \\
\hline H1 & $12 / 31 / 1971$ & $\mathrm{P} 2$ & $7 / 1 / 1996$ \\
\hline $\mathrm{H} 2$ & $12 / 31 / 1971$ & P3 & $7 / 1 / 1996$ \\
\hline H3 & $12 / 31 / 1971$ & $\mathrm{P} 4$ & $7 / 1 / 1996$ \\
\hline $\mathrm{H} 4$ & $6 / 1 / 1972$ & $\mathrm{C} 2$ & $1 / 1 / 1997$ \\
\hline НВ & $4 / 21 / 1988$ & $\mathrm{C} 3$ & $1 / 1 / 1997$ \\
\hline I1 & $1 / 1 / 1994$ & L9 & $1 / 1 / 1997$ \\
\hline $\mathrm{I} 2$ & $1 / 1 / 1994$ & D4 & $3 / 10 / 1997$ \\
\hline I3 & $1 / 1 / 1994$ & N8 & $4 / 8 / 1997$ \\
\hline I4 & $1 / 1 / 2001$ & L3 & $5 / 1 / 1997$ \\
\hline I5 & $1 / 1 / 2002$ & $\mathrm{P} 5$ & $8 / 1 / 1997$ \\
\hline I7 & $1 / 1 / 2002$ & A3 & $8 / 14 / 1997$ \\
\hline I8 & $1 / 1 / 2001$ & B8 & $1 / 1 / 1998$ \\
\hline I9 & $1 / 1 / 2001$ & B9 & $1 / 1 / 1998$ \\
\hline IA & $1 / 2 / 2002$ & F3 & $1 / 1 / 1998$ \\
\hline IB & $2 / 21 / 2002$ & F 4 & $1 / 1 / 1998$ \\
\hline $\mathrm{J} 1$ & $1 / 1 / 1988$ & G7 & $1 / 1 / 1998$ \\
\hline $\mathrm{J} 2$ & $1 / 1 / 1988$ & $\mathrm{~K} 2$ & $1 / 1 / 1998$ \\
\hline J3 & $1 / 15 / 1999$ & K3 & $1 / 1 / 1998$ \\
\hline J5 & $1 / 1 / 1988$ & K4 & $1 / 1 / 1998$ \\
\hline J6 & $1 / 1 / 1988$ & K5 & $1 / 1 / 1998$ \\
\hline $\mathrm{J} 7$ & $1 / 1 / 1988$ & K6 & $1 / 1 / 1998$ \\
\hline
\end{tabular}

Continued on next page... 


\begin{tabular}{|c|c|c|c|}
\hline \multicolumn{2}{|c|}{ sorted by qual } & \multicolumn{2}{|c|}{ sorted by date } \\
\hline qual & date & qual & date \\
\hline J8 & $1 / 1 / 2001$ & K7 & $1 / 1 / 1998$ \\
\hline J9 & $1 / 1 / 2000$ & $\mathrm{P} 7$ & $1 / 1 / 1998$ \\
\hline JA & $1 / 1 / 2005$ & $\mathrm{P} 8$ & $1 / 1 / 1998$ \\
\hline $\mathrm{JL}$ & $4 / 1 / 1996$ & N9 & $2 / 1 / 1998$ \\
\hline $\mathrm{JM}$ & $12 / 31 / 1971$ & Q1 & $2 / 7 / 1998$ \\
\hline $\mathrm{JN}$ & $1 / 1 / 1973$ & 2 & $4 / 11 / 1998$ \\
\hline $\mathrm{K} 1$ & $1 / 1 / 1994$ & J3 & $1 / 15 / 1999$ \\
\hline $\mathrm{K} 2$ & $1 / 1 / 1998$ & 13 & $1 / 1 / 2000$ \\
\hline K3 & $1 / 1 / 1998$ & $4 \mathrm{~A}$ & $1 / 1 / 2000$ \\
\hline K4 & $1 / 1 / 1998$ & $4 \mathrm{~B}$ & $1 / 1 / 2000$ \\
\hline K5 & $1 / 1 / 1998$ & $4 \mathrm{C}$ & $1 / 1 / 2000$ \\
\hline K6 & $1 / 1 / 1998$ & $4 \mathrm{D}$ & $1 / 1 / 2000$ \\
\hline $\mathrm{K} 7$ & $1 / 1 / 1998$ & $4 \mathrm{E}$ & $1 / 1 / 2000$ \\
\hline K9 & $1 / 1 / 1994$ & $4 \mathrm{~F}$ & $1 / 1 / 2000$ \\
\hline KA & $1 / 1 / 2006$ & $4 \mathrm{I}$ & $1 / 1 / 2000$ \\
\hline KB & $1 / 1 / 2006$ & $4 \mathrm{~J}$ & $1 / 1 / 2000$ \\
\hline $\mathrm{KC}$ & $1 / 1 / 2006$ & $4 \mathrm{~K}$ & $1 / 1 / 2000$ \\
\hline L1 & $5 / 1 / 1992$ & $4 \mathrm{M}$ & $1 / 1 / 2000$ \\
\hline L2 & $1 / 1 / 1993$ & $4 \mathrm{~N}$ & $1 / 1 / 2000$ \\
\hline L3 & $5 / 1 / 1997$ & $4 \mathrm{P}$ & $1 / 1 / 2000$ \\
\hline L4 & $12 / 1 / 1980$ & J9 & $1 / 1 / 2000$ \\
\hline L5 & $6 / 1 / 2005$ & $\mathrm{~N} 4$ & $1 / 1 / 2000$ \\
\hline L6 & $4 / 1 / 2006$ & O7 & $1 / 1 / 2000$ \\
\hline L8 & $1 / 1 / 1972$ & Q2 & $1 / 1 / 2000$ \\
\hline L9 & $1 / 1 / 1997$ & Q3 & $1 / 1 / 2000$ \\
\hline M1 & $1 / 1 / 1992$ & EX & $8 / 1 / 2000$ \\
\hline M2 & $10 / 1 / 1992$ & I4 & $1 / 1 / 2001$ \\
\hline M3 & $3 / 1 / 1993$ & I8 & $1 / 1 / 2001$ \\
\hline M4 & $12 / 31 / 1971$ & I9 & $1 / 1 / 2001$ \\
\hline M8 & $1 / 1 / 2001$ & $\mathrm{~J} 8$ & $1 / 1 / 2001$ \\
\hline M9 & $1 / 1 / 1995$ & M8 & $1 / 1 / 2001$ \\
\hline N1 & $1 / 1 / 1996$ & O1 & $1 / 1 / 2001$ \\
\hline $\mathrm{N} 2$ & $7 / 1 / 1994$ & $\mathrm{O} 2$ & $1 / 1 / 2001$ \\
\hline N3 & $1 / 1 / 1996$ & O3 & $1 / 1 / 2001$ \\
\hline N4 & $1 / 1 / 2000$ & $\mathrm{O} 4$ & $1 / 1 / 2001$ \\
\hline N6 & $5 / 1 / 1996$ & O5 & $1 / 1 / 2001$ \\
\hline N7 & $5 / 1 / 1996$ & O6 & $1 / 1 / 2001$ \\
\hline N8 & $4 / 8 / 1997$ & OK & $1 / 1 / 2001$ \\
\hline N9 & $2 / 1 / 1998$ & Q4 & $1 / 1 / 2001$ \\
\hline $\mathrm{NU}$ & $1 / 1 / 2005$ & Q9 & $2 / 19 / 2001$ \\
\hline O1 & $1 / 1 / 2001$ & $4 \mathrm{R}$ & $4 / 1 / 2001$ \\
\hline $\mathrm{O} 2$ & $1 / 1 / 2001$ & $3 \mathrm{~A}$ & $1 / 1 / 2002$ \\
\hline O3 & $1 / 1 / 2001$ & I5 & $1 / 1 / 2002$ \\
\hline $\mathrm{O} 4$ & $1 / 1 / 2001$ & I7 & $1 / 1 / 2002$ \\
\hline O5 & $1 / 1 / 2001$ & IA & $1 / 2 / 2002$ \\
\hline O6 & $1 / 1 / 2001$ & IB & $2 / 21 / 2002$ \\
\hline O7 & $1 / 1 / 2000$ & O8 & $7 / 1 / 2002$ \\
\hline O8 & $7 / 1 / 2002$ & $3 \mathrm{~T}$ & $1 / 1 / 2003$ \\
\hline O9 & $6 / 1 / 2003$ & O9 & $6 / 1 / 2003$ \\
\hline OJ & $1 / 1 / 2004$ & OJ & $1 / 1 / 2004$ \\
\hline OK & $1 / 1 / 2001$ & $3 \mathrm{~B}$ & $1 / 1 / 2005$ \\
\hline $\mathrm{P} 1$ & $7 / 1 / 1996$ & $3 \mathrm{E}$ & $1 / 1 / 2005$ \\
\hline $\mathrm{P} 2$ & $7 / 1 / 1996$ & $3 \mathrm{G}$ & $1 / 1 / 2005$ \\
\hline P3 & $7 / 1 / 1996$ & $3 \mathrm{~J}$ & $1 / 1 / 2005$ \\
\hline $\mathrm{P} 4$ & $7 / 1 / 1996$ & $\mathrm{CX}$ & $1 / 1 / 2005$ \\
\hline P5 & $8 / 1 / 1997$ & JA & $1 / 1 / 2005$ \\
\hline P6 & $1 / 1 / 1984$ & $\mathrm{NU}$ & $1 / 1 / 2005$ \\
\hline $\mathrm{P} 7$ & $1 / 1 / 1998$ & Y1 & $1 / 1 / 2005$ \\
\hline P8 & $1 / 1 / 1998$ & Y4 & $1 / 1 / 2005$ \\
\hline P9 & $1 / 1 / 1981$ & Y5 & $1 / 1 / 2005$ \\
\hline Q1 & $2 / 7 / 1998$ & Y6 & $1 / 1 / 2005$ \\
\hline Q2 & $1 / 1 / 2000$ & $\mathrm{Y} 7$ & $1 / 1 / 2005$ \\
\hline Q3 & $1 / 1 / 2000$ & Y8 & $1 / 1 / 2005$ \\
\hline
\end{tabular}

Continued on next page. 


\begin{tabular}{|c|c|c|c|}
\hline \multicolumn{2}{|c|}{ sorted by qual } & \multicolumn{2}{|c|}{ sorted by date } \\
\hline qual & date & qual & date \\
\hline Q4 & $1 / 1 / 2001$ & Y9 & $1 / 1 / 2005$ \\
\hline Q5 & $1 / 1 / 1994$ & $\mathrm{Z3}$ & $1 / 1 / 2005$ \\
\hline Q6 & $5 / 1 / 1996$ & $\mathrm{ZC}$ & $1 / 1 / 2005$ \\
\hline Q7 & $5 / 1 / 1996$ & $\mathrm{ZD}$ & $1 / 1 / 2005$ \\
\hline Q8 & $5 / 1 / 1996$ & $\mathrm{ZJ}$ & $1 / 1 / 2005$ \\
\hline Q9 & $2 / 19 / 2001$ & $\mathrm{ZM}$ & $1 / 1 / 2005$ \\
\hline $\mathrm{Y} 1$ & $1 / 1 / 2005$ & L5 & $6 / 1 / 2005$ \\
\hline Y4 & $1 / 1 / 2005$ & $\mathrm{Z} 1$ & $9 / 1 / 2005$ \\
\hline Y5 & $1 / 1 / 2005$ & $\mathrm{Z} 4$ & $11 / 1 / 2005$ \\
\hline Y6 & $1 / 1 / 2005$ & $4 \mathrm{~L}$ & $1 / 1 / 2006$ \\
\hline $\mathrm{Y} 7$ & $1 / 1 / 2005$ & $\mathrm{BB}$ & $1 / 1 / 2006$ \\
\hline Y8 & $1 / 1 / 2005$ & GD & $1 / 1 / 2006$ \\
\hline Y9 & $1 / 1 / 2005$ & GT & $1 / 1 / 2006$ \\
\hline $\mathrm{Z} 1$ & $9 / 1 / 2005$ & KA & $1 / 1 / 2006$ \\
\hline $\mathrm{Z3}$ & $1 / 1 / 2005$ & $\mathrm{~KB}$ & $1 / 1 / 2006$ \\
\hline $\mathrm{Z} 4$ & $11 / 1 / 2005$ & $\mathrm{KC}$ & $1 / 1 / 2006$ \\
\hline $\mathrm{ZC}$ & $1 / 1 / 2005$ & $\mathrm{BE}$ & $1 / 5 / 2006$ \\
\hline $\mathrm{ZD}$ & $1 / 1 / 2005$ & $\mathrm{BD}$ & $2 / 1 / 2006$ \\
\hline $\mathrm{ZJ}$ & $1 / 1 / 2005$ & $\mathrm{BK}$ & $2 / 1 / 2006$ \\
\hline $\mathrm{ZM}$ & $1 / 1 / 2005$ & L6 & $4 / 1 / 2006$ \\
\hline
\end{tabular}


Table 4: Minimal and maximal contribution basis, 1972-2007, in $€$

\begin{tabular}{lrr}
\hline \hline year & min & max \\
\hline 1972 & 661 & 8,852 \\
1973 & 661 & 9,615 \\
1974 & 926 & 10,683 \\
1975 & 1,058 & 11,904 \\
1976 & 1,058 & 13,430 \\
1977 & 1,526 & 15,261 \\
1978 & 1,632 & 17,093 \\
1979 & 1,738 & 18,924 \\
1980 & 1,835 & 19,840 \\
1981 & 1,929 & 20,755 \\
1982 & 2,030 & 21,976 \\
1983 & 2,142 & 23,197 \\
1984 & 2,227 & 24,418 \\
1985 & 2,300 & 25,029 \\
1986 & 2,395 & 26,249 \\
1987 & 2,494 & 26,860 \\
1988 & 2,571 & 28,081 \\
1989 & 2,638 & 28,691 \\
1990 & 2,704 & 29,302 \\
1991 & 2,820 & 30,523 \\
1992 & 2,975 & 32,354 \\
1993 & 3,156 & 34,185 \\
1994 & 3,345 & 36,627 \\
1995 & 3,512 & 38,458 \\
1996 & 3,663 & 39,679 \\
1997 & 3,805 & 41,511 \\
1998 & 3,897 & 42,732 \\
1999 & 3,967 & 43,342 \\
2000 & 4,046 & 43,953 \\
2001 & 4,147 & 45,173 \\
2002 & 4,222 & 45,780 \\
2003 & 4,331 & 47,040 \\
2004 & 4,427 & 48,300 \\
2005 & 4,528 & 50,820 \\
2006 & 4,664 & 52,500 \\
2007 & 4,776 & 53,760 \\
\hline & & \\
1989
\end{tabular}


Table 5: Codelist for the variable stsl

\begin{tabular}{|c|c|}
\hline code & country \\
\hline 001 & Österreich \\
\hline 002 & Deutschland \\
\hline 003 & Schweiz \\
\hline 004 & Liechtenstein \\
\hline 005 & Italien \\
\hline 006 & ehemaliges Jugoslawien \\
\hline 007 & Ungarn \\
\hline 008 & ehemalige Tschechoslowakei \\
\hline 009 & Tschechische Republik \\
\hline 010 & Slowakei \\
\hline 012 & Deutschland (ehem. DDR) \\
\hline 013 & Dänemark \\
\hline 014 & Niederlande \\
\hline 015 & Belgien \\
\hline 016 & Luxemburg \\
\hline 017 & Frankreich \\
\hline 018 & Spanien \\
\hline 019 & Portugal \\
\hline 022 & Andorra \\
\hline 023 & Monaco \\
\hline 024 & San Marino \\
\hline 025 & Vatikanstadt \\
\hline 030 & Malta \\
\hline 031 & Albanien \\
\hline 032 & Griechenland \\
\hline 033 & Türkei \\
\hline 034 & Bulgarien \\
\hline 035 & Rumänien \\
\hline 036 & ehemalige Sowjetunion \\
\hline 037 & Polen \\
\hline 040 & Finnland \\
\hline 041 & Schweden \\
\hline 042 & Norwegen \\
\hline 043 & Vereinigtes Königreich Großbritannien \\
\hline 044 & Irland \\
\hline 045 & Island \\
\hline 046 & Estland \\
\hline 047 & Lettland \\
\hline 048 & Litauen \\
\hline 051 & Slowenien \\
\hline 052 & Kroatien \\
\hline 053 & Bosnien und Herzegovina \\
\hline 054 & Mazedonien \\
\hline 055 & Serbien und Montenegro \\
\hline 060 & Russische Föderation \\
\hline
\end{tabular}

Continued on next page... 


\begin{tabular}{|c|c|}
\hline code & country \\
\hline 061 & Belarus (Weißrussland) \\
\hline 062 & Ukraine \\
\hline 063 & Armenien \\
\hline 064 & Aserbaidschan \\
\hline 065 & Kasachstan \\
\hline 066 & Moldova \\
\hline 067 & Georgien \\
\hline 068 & Färöer \\
\hline 069 & Gibraltar \\
\hline 070 & Svalbard und Jan Mayen \\
\hline 100 & Asien, nicht näher zuordenbar \\
\hline 101 & Zypern \\
\hline 102 & Israel \\
\hline 103 & Libanon \\
\hline 104 & Jordanien \\
\hline 105 & Syrien \\
\hline 106 & Irak \\
\hline 107 & Kuwait \\
\hline 108 & Saudi-Arabien \\
\hline 109 & Jemen (ehem.demokr.VR) \\
\hline 110 & Jemen \\
\hline 115 & Bahrain \\
\hline 116 & Katar \\
\hline 117 & Vereinigte Arabische Emirate \\
\hline 118 & Oman \\
\hline 119 & Iran \\
\hline 120 & Afghanistan \\
\hline 125 & Pakistan \\
\hline 126 & Indien \\
\hline 127 & Nepal \\
\hline 128 & China \\
\hline 129 & Mongolei \\
\hline 130 & Bangladesch \\
\hline 135 & Sikkim (zu Indien) \\
\hline 136 & Bhutan \\
\hline 137 & Myanmar \\
\hline 138 & Thailand \\
\hline 139 & Kambodscha \\
\hline 140 & Laos \\
\hline 144 & Brunei \\
\hline 145 & Malaysia \\
\hline 146 & Singapur \\
\hline 148 & Vietnam \\
\hline 149 & Macau (zu Portugal) \\
\hline 150 & Hongkong \\
\hline 155 & Korea, Dem. VR \\
\hline
\end{tabular}




\begin{tabular}{|c|c|}
\hline code & country \\
\hline 156 & Korea, Rep. \\
\hline 157 & Malediven \\
\hline 158 & Sri Lanka \\
\hline 159 & Indonesien \\
\hline 160 & Timor (zu Indonesien) \\
\hline 161 & Philippinen \\
\hline 165 & Republik China (Taiwan) \\
\hline 166 & Japan \\
\hline 170 & Usbekistan \\
\hline 171 & Tadschikistan \\
\hline 172 & Turkmenistan \\
\hline 173 & Kirgisistan \\
\hline 174 & Timor-Leste \\
\hline 175 & Taiwan \\
\hline 200 & Afrika, nicht näher zuordenbar \\
\hline 201 & Marokko \\
\hline 202 & Algerien \\
\hline 203 & Tunesien \\
\hline 204 & Libyen \\
\hline 205 & Ägypten \\
\hline 206 & Sahara (dzt. zu Marokko) \\
\hline 207 & Mauretanien \\
\hline 208 & Senegal \\
\hline 209 & Gambia \\
\hline 210 & Mali \\
\hline 215 & Burkina Faso \\
\hline 216 & Niger \\
\hline 217 & Tschad \\
\hline 218 & Sudan \\
\hline 219 & Äthiopien \\
\hline 220 & Somalia \\
\hline 221 & Eritrea \\
\hline 225 & Dschibuti \\
\hline 226 & Guinea-Bissau \\
\hline 227 & Guinea \\
\hline 228 & Sierra Leone \\
\hline 229 & Liberia \\
\hline 230 & Cote d` Ivoire (Elfenbeink.) \\
\hline 235 & Ghana \\
\hline 236 & Togo \\
\hline 237 & Benin \\
\hline 238 & Nigeria \\
\hline 239 & Kamerun \\
\hline 240 & Zentralafrikanische Republik \\
\hline 245 & Äquatorialguinea \\
\hline 246 & Gabun \\
\hline
\end{tabular}

Continued on next page... 


\begin{tabular}{|c|c|}
\hline code & country \\
\hline 247 & Kongo (Brazzaville) \\
\hline 248 & Kongo, Dem. Rep. \\
\hline 249 & Uganda \\
\hline 250 & Ruanda \\
\hline 255 & Burundi \\
\hline 256 & Tansania \\
\hline 257 & Kenia \\
\hline 258 & Angola \\
\hline 259 & Sambia \\
\hline 260 & Malawi \\
\hline 265 & Mosambik \\
\hline 266 & Namibia \\
\hline 268 & Südafrika \\
\hline 269 & Botsuana \\
\hline 270 & Simbabwe \\
\hline 275 & Lesotho \\
\hline 276 & Swasiland \\
\hline 277 & Madagaskar \\
\hline 278 & Mauritius \\
\hline 280 & Seychellen \\
\hline 281 & Kap Verde \\
\hline 282 & Komoren \\
\hline 283 & Sao Tome und Principe \\
\hline 284 & Französische Südgebiete \\
\hline 286 & Westsahara \\
\hline 287 & Brit. Territ. im Ind. Ozean \\
\hline 288 & Mayotte \\
\hline 289 & Reunion \\
\hline 290 & St. Helena \\
\hline 300 & Amerika, nicht näher zuordenbar \\
\hline 301 & Kanada \\
\hline 302 & Vereinigte Staaten (USA) \\
\hline 303 & Grönland (zu Dänemark) \\
\hline 304 & Mexico \\
\hline 307 & Guatemala \\
\hline 308 & Belize \\
\hline 309 & Honduras \\
\hline 310 & El Salvator \\
\hline 315 & Nicaragua \\
\hline 316 & Costa Rica \\
\hline 317 & Panama \\
\hline 318 & Kuba \\
\hline 319 & Jamaika \\
\hline 320 & Haiti \\
\hline 325 & Dominikanische Republik \\
\hline 326 & Puerto Rico (zu USA) \\
\hline
\end{tabular}




\begin{tabular}{|c|c|}
\hline code & country \\
\hline 327 & Bahamas \\
\hline 328 & Bermuda \\
\hline 329 & Westindische assoz. Staaten \\
\hline 330 & Guadeloupe (zu Frankreich) \\
\hline 331 & Martinique (zu Frankreich) \\
\hline 332 & Barbados \\
\hline 333 & Niederländische Antillen \\
\hline 337 & Kolumbien \\
\hline 338 & Venezuela \\
\hline 339 & Guyana \\
\hline 340 & Suriname (zu Niederlande) \\
\hline 345 & Französisch Guyana \\
\hline 346 & Trinidad und Tobago \\
\hline 347 & Ecuador \\
\hline 348 & Peru \\
\hline 349 & Brasilien \\
\hline 350 & Bolivien \\
\hline 355 & Paraguay \\
\hline 356 & Chile \\
\hline 357 & Argentinien \\
\hline 358 & Uruguay \\
\hline 361 & Falkland-Inseln (zu GB) \\
\hline 362 & Süd-Georgien \\
\hline 363 & Süd-Sandwich-Inseln \\
\hline 381 & Grenada \\
\hline 382 & Dominica \\
\hline 383 & St. Lucia \\
\hline 384 & St. Vincent und Grenadinen \\
\hline 385 & Antigua und Barbuda \\
\hline 386 & St. Kitts und Nevis \\
\hline 387 & Aruba \\
\hline 388 & Anguilla \\
\hline 389 & Kaimaninseln \\
\hline 390 & Montserrat \\
\hline 391 & St. Pierre und Miquelon \\
\hline 392 & Turks- und Caicosinseln \\
\hline 393 & Britische Jungferninseln \\
\hline 394 & Amerikan. Jungferninseln \\
\hline 400 & Australien/Ozeanien div. \\
\hline 401 & Australien \\
\hline 405 & Neuseeland \\
\hline 406 & Palau \\
\hline 410 & Marianen (zu USA) \\
\hline 411 & Guam (zu USA) \\
\hline 412 & Hawaii (zu USA) \\
\hline 413 & Marshall-Inseln \\
\hline
\end{tabular}

Continued on next page... 


\begin{tabular}{|c|c|}
\hline code & country \\
\hline 414 & Mikronesien \\
\hline 415 & Nauru \\
\hline 416 & Belau (Palau-Inseln) \\
\hline 420 & Kiribati (Gilbert-Inseln) \\
\hline 421 & Salomonen \\
\hline 422 & Tuvalu (Ellice-Inseln) \\
\hline 423 & Vanuatu (neue Hebriden) \\
\hline 424 & Neukaledonien (zu F) \\
\hline 427 & Fidschi \\
\hline 428 & Samoa \\
\hline 429 & Tonga \\
\hline 431 & Papua-Neuguinea \\
\hline 432 & Kokosinseln (Australien) \\
\hline 433 & Weihnachtsinseln (Australien) \\
\hline 434 & Cookinseln \\
\hline 435 & Heard und die McDonaldinseln \\
\hline 436 & Norfolkinsel \\
\hline 437 & Niue \\
\hline 438 & Pitcairninseln \\
\hline 439 & Tokelau \\
\hline 440 & Kleinere Am. Überseeinseln \\
\hline 441 & Wallis und Futuna \\
\hline 442 & Amerikanische Samoa \\
\hline 443 & Französisch-Polynesien \\
\hline 500 & Antarktis \\
\hline 501 & Bouvetinsel \\
\hline
\end{tabular}


Table 6: Codelist for the variable bundesland

\begin{tabular}{ll}
\hline \hline code & province \\
\hline 0 & multiple provinces \\
1 & Vienna \\
2 & Lower Austria \\
3 & Burgenland \\
4 & Upper Austria \\
5 & Styria \\
6 & Carinthia \\
7 & Salzburg \\
8 & Tyrol \\
9 & Vorarlberg \\
10 & outside of Austria \\
\hline
\end{tabular}


Table 7: Codelist for the variable beot

\begin{tabular}{|c|c|}
\hline code & location \\
\hline \multicolumn{2}{|c|}{$\mathbf{1}^{\text {st }}$ digit } \\
\hline blank & Österreich \\
\hline 1 & Italien \\
\hline 2 & Schweiz \\
\hline 3 & Liechtenstein \\
\hline 4 & Bundesrepublik Deutschland (BRD) \\
\hline 5 & Tschechien \\
\hline 6 & Ungarn \\
\hline 7 & Slowenien \\
\hline 8 & Slowakei \\
\hline 9 & Andere Staaten \\
\hline \multicolumn{2}{|c|}{$\mathbf{2}^{\text {nd }}$ digit } \\
\hline 0 & keine Zuordnung möglich \\
\hline 1 & Wien \\
\hline 2 & Niederösterreich \\
\hline 3 & Burgenland \\
\hline 4 & Oberösterreich \\
\hline 5 & Steiermark \\
\hline 6 & Kärnten \\
\hline 7 & Salzburg \\
\hline 8 & Tirol \\
\hline 9 & Vorarlberg \\
\hline \multicolumn{2}{|c|}{$\mathbf{3}^{r d}$ and $4^{\text {th }}$ digit } \\
\hline \multicolumn{2}{|c|}{ if $1^{\text {st }}$ digit $=$ blank and $2^{\text {nd }}$ digit $=1$} \\
\hline 01 & Innere Stadt \\
\hline 02 & Leopoldstadt \\
\hline 03 & Landstraße \\
\hline 04 & Wieden \\
\hline 05 & Margareten \\
\hline 06 & Mariahilf \\
\hline 07 & Neubau \\
\hline 08 & Josefstadt \\
\hline 09 & Alsergrund \\
\hline 10 & Favoriten \\
\hline 11 & Simmering \\
\hline 12 & Meidling \\
\hline 13 & Hietzing \\
\hline 14 & Penzing \\
\hline 15 & Rudolfsheim - Fünfhaus \\
\hline 16 & Ottakring \\
\hline 17 & Hernals \\
\hline 18 & Währing \\
\hline 19 & Döbling \\
\hline
\end{tabular}

Continued on next page... 


\begin{tabular}{|c|c|}
\hline code & location \\
\hline 20 & Brigittenau \\
\hline 21 & Floridsdorf \\
\hline 22 & Donaustadt \\
\hline 23 & Liesing \\
\hline \multicolumn{2}{|c|}{ if $1^{\text {st }}$ digit $=$ blank and $2^{\text {nd }}$ digit $=2$} \\
\hline 01 & Amstetten \\
\hline 02 & Baden \\
\hline 03 & Bruck/Leitha \\
\hline 05 & Gänserndorf \\
\hline 06 & Gmünd \\
\hline 07 & Hollabrunn \\
\hline 08 & Horn \\
\hline 09 & Korneuburg \\
\hline 10 & Krems \\
\hline 11 & Lilienfeld \\
\hline 12 & Melk \\
\hline 13 & Mistelbach \\
\hline 14 & Mödling \\
\hline 15 & Neunkirchen \\
\hline 19 & Scheibbs \\
\hline 20 & Tulln \\
\hline 21 & Waidhofen/Thaya \\
\hline 22 & Wr. Neustadt \\
\hline 23 & Wien-Umgebung \\
\hline 24 & Zwettl \\
\hline \multicolumn{2}{|c|}{ if $1^{\text {st }}$ digit $=$ blank and $2^{\text {nd }}$ digit $=3$} \\
\hline 01 & Eisenstadt-Umgebung \\
\hline 02 & Neusiedl \\
\hline 03 & Mattersburg \\
\hline 04 & Oberpullendorf \\
\hline 05 & Oberwart \\
\hline 06 & Güssing \\
\hline 07 & Jennersdorf \\
\hline \multicolumn{2}{|c|}{ if $1^{\text {st }}$ digit $=$ blank and $2^{\text {nd }}$ digit $=4$} \\
\hline 01 & Braunau \\
\hline 02 & Eferding \\
\hline 03 & Freistadt \\
\hline 04 & Gmunden \\
\hline 05 & Grieskirchen \\
\hline 06 & Kirchdorf \\
\hline 07 & Linz-Land \\
\hline 08 & Perg \\
\hline 09 & Ried i. I. \\
\hline 10 & Rohrbach \\
\hline 11 & Schärding \\
\hline
\end{tabular}

Continued on next page... 


\begin{tabular}{|c|c|}
\hline code & location \\
\hline 12 & Steyr-Land \\
\hline 13 & Urfahr-Umgebung \\
\hline 14 & Vöcklabruck \\
\hline 15 & Wels-Land \\
\hline 16 & Wels-Stadt \\
\hline 17 & Steyr-Stadt \\
\hline 18 & Linz-Stadt \\
\hline \multicolumn{2}{|c|}{ if $1^{\text {st }}$ digit $=$ blank and $2^{\text {nd }}$ digit $=5$} \\
\hline 02 & Bruck/Mur \\
\hline 03 & Deutschlandsberg \\
\hline 04 & Feldbach \\
\hline 05 & Fürstenfeld \\
\hline 06 & Graz-Umgebung \\
\hline 07 & Hartberg \\
\hline 08 & Judenburg \\
\hline 09 & Knittelfeld \\
\hline 10 & Leibnitz \\
\hline 11 & Leoben \\
\hline 12 & Liezen \\
\hline 13 & Mürzzuschlag \\
\hline 14 & Murau \\
\hline 15 & Radkersburg \\
\hline 16 & Voitsberg \\
\hline 17 & Weiz \\
\hline \multicolumn{2}{|c|}{ if $1^{\text {st }}$ digit $=$ blank and $2^{\text {nd }}$ digit $=6$} \\
\hline 01 & Klagenfurt \\
\hline 02 & Villach \\
\hline 03 & Hermagor \\
\hline 04 & Klagenfurt-Land \\
\hline 05 & St. Veit an der Glan \\
\hline 06 & Spittal an der Drau \\
\hline 07 & Villach-Land \\
\hline 08 & Völkermarkt \\
\hline 09 & Wolfsberg \\
\hline 10 & Feldkirchen \\
\hline \multicolumn{2}{|c|}{ if $1^{\text {st }}$ digit $=$ blank and $2^{\text {nd }}$ digit $=7$} \\
\hline 00 & Salzburg-Stadt \\
\hline 01 & Salzburg-Umgebung \\
\hline 02 & Hallein \\
\hline 03 & St. Johann i. P. \\
\hline 04 & Zell am See \\
\hline 05 & Tamsweg \\
\hline \multicolumn{2}{|c|}{ if $1^{\text {st }}$ digit $=$ blank and $2^{\text {nd }}$ digit $=8$} \\
\hline 01 & Innsbruck-Stadt \\
\hline
\end{tabular}

Continued on next page... 


\begin{tabular}{ll}
$\ldots$ table 7 & continued \\
\hline \hline code & location \\
\hline 02 & Imst \\
03 & Innsbruck-Land \\
04 & Kitzbühel \\
05 & Kufstein \\
06 & Landeck \\
07 & Lienz \\
08 & Reutte \\
09 & Schwaz \\
if $1^{\text {st }}$ digit $=$ blank and $2^{\text {nd }}$ digit $=9$ \\
01 & Bludenz \\
02 & Bregenz \\
03 & Dornbirn \\
04 & Feldkirch \\
$\mathbf{5}^{\text {th }}$ and & $\mathbf{6}^{\text {th }}$ digit \\
- & non-informative \\
\hline
\end{tabular}


Table 8: Codelist for the variable $n u t s$

\begin{tabular}{llll}
\hline \hline $\mathbf{1}^{s t}$ & $\mathbf{2}^{\text {nd }}$ & $\mathbf{3}^{\text {rd }}$ & region \\
& digit & \\
\hline
\end{tabular}

\section{Ostösterreich}

Burgenland

\begin{tabular}{cccl}
1 & 1 & 1 & Mittelburgenland \\
1 & 1 & 2 & Nordburgenland \\
1 & 1 & 3 & Südburgenland \\
\multicolumn{4}{c}{ Niederösterreich }
\end{tabular}

Niederösterreich

\begin{tabular}{|c|c|c|c|}
\hline 1 & 2 & 1 & Mostviertel - Eisenwurzen \\
\hline 1 & 2 & 2 & Niederösterreich-Süd \\
\hline 1 & 2 & 3 & Sankt Pölten \\
\hline 1 & 2 & 4 & Waldviertel \\
\hline 1 & 2 & 5 & Weinviertel \\
\hline 1 & 2 & 6 & Wiener Umland - Nordteil \\
\hline 1 & 2 & 7 & Wiener Umland - Südteil \\
\hline \multicolumn{4}{|c|}{ Wien } \\
\hline 1 & & 1 & Wien \\
\hline
\end{tabular}

\section{Südösterreich}

\begin{tabular}{llll}
\multicolumn{3}{c}{ Kärnten } \\
2 & 1 & 1 & Klagenfurt - Villach \\
2 & 1 & 2 & Oberkärnten \\
2 & 1 & 3 & Unterkärnten \\
\multicolumn{3}{c}{ Steiermark } & \\
2 & 2 & 1 & Graz \\
2 & 2 & 2 & Liezen \\
2 & 2 & 3 & Östliche Obersteiermark \\
2 & 2 & 4 & Oststeiermark \\
2 & 2 & 5 & West- und Südsteiermark \\
2 & 2 & 6 & Westliche Obersteiermark
\end{tabular}

\section{Westösterreich}

Oberösterreich

\begin{tabular}{llll}
3 & 1 & 1 & Innviertel \\
3 & 1 & 2 & Linz - Wels \\
3 & 1 & 3 & Mühlviertel \\
3 & 1 & 4 & Steyr - Kirchdorf \\
3 & 1 & 5 & Traunviertel \\
\multicolumn{2}{c}{ Salzburg } & & \\
3 & 2 & 1 & Lungau \\
3 & 2 & 2 & Pinzgau - Pongau \\
3 & 2 & 3 & Salzburg und Umgebung \\
\multicolumn{2}{l}{ Tirol } & & \\
3 & 3 & 1 & Außerfern \\
3 & 3 & 2 & Innsbruck \\
\hline \multicolumn{5}{l}{} \\
\end{tabular}




\begin{tabular}{llll}
\multicolumn{5}{c}{$\ldots$ table 8 continued } \\
\hline \hline $\mathbf{1}^{\text {st }}$ & $\mathbf{2}^{\text {nd }}$ & $\mathbf{3}^{\text {rd }}$ & region \\
& digit & & \\
\hline 3 & 3 & 3 & Osttirol \\
3 & 3 & 4 & Tiroler Oberland \\
3 & 3 & 5 & Tiroler Unterland \\
\multicolumn{2}{c}{ Vorarlberg } & & \\
3 & 4 & 1 & Bludenz - Bregenzer Wald \\
3 & 4 & 2 & Rheintal - Bodenseegebiet \\
\hline
\end{tabular}


Table 9: Codelist for the variable industry_ 15

\begin{tabular}{|c|c|}
\hline code & industry affiliation \\
\hline 1 & Landwirtschaft, Fischerei, Forstwirtschaft, Jagd \\
\hline 2 & Elektrizitätsversorgung, Gasversorgung, Wärmeversorgung, Wasserversorgung \\
\hline 3 & $\begin{array}{l}\text { Kohlenbergbau, Erzbergbau, Erdöl- und Erdgasbergbau, Salzbergbau, Magnesit- } \\
\text { bergbau, Sonstiger Bergbau, Torfabbau, Steine(Erden)gewinnung }\end{array}$ \\
\hline 4 & Erzeugung von Sachgütern \\
\hline 5 & Hoch- und Tiefbau, Ausbau- und Bauhilfsgewerbe, Bauinstallation \\
\hline 6 & Großhandel, Einzelhandel, Lagerung und Aufbewahrung \\
\hline 7 & Beherbergungs- und Gaststättenwesen \\
\hline 8 & $\begin{array}{l}\text { Straßenverkehr, Eisenbahn- und Seilbahnverkehr, Schiffahrt, Luftverkehr, Trans- } \\
\text { port in Rohrleitungen, Spedition, Nachrichtenübermittlung }\end{array}$ \\
\hline 9 & $\begin{array}{l}\text { Geld- und Kreditwesen, Privatversicherung, Realitätenwesen, Rechts- und } \\
\text { Wirtschaftsdienste }\end{array}$ \\
\hline 10 & Körperpflege und Reinigung, Bestattungswesen \\
\hline 11 & Kunst, Unterhaltung, Sport \\
\hline 12 & Gesundheits- und Fürsorgewesen \\
\hline 13 & Unterrichts- und Forschungswesen \\
\hline 14 & $\begin{array}{l}\text { Sozialversicherungsträger, Interessenvertretungen, Einrichtungen der Gebi- } \\
\text { etskörperschaften }\end{array}$ \\
\hline 15 & Haushaltung und Hauswartung \\
\hline
\end{tabular}


Table 10: Codelist for the variable $w i k l_{-} 25$

\begin{tabular}{|c|c|}
\hline code & industry \\
\hline 1 & Landwirtschaft, Fischerei, Forstwirtschaft, Jagd \\
\hline 2 & Elektrizitätsversorgung, Gasversorgung, Wärmeversorgung, Wasserversorgung \\
\hline 3 & $\begin{array}{l}\text { Kohlenbergbau, Erzbergbau, Erdöl- und Erdgasbergbau, Salzbergbau, Magnesit- } \\
\text { bergbau, Sonstiger Bergbau, Torfabbau, Steine(Erden)gewinnung }\end{array}$ \\
\hline 4 & $\begin{array}{l}\text { Erzeugung von Nahrungs- und Genuflmitteln, Erzeugung von Getränken, } \\
\text { Tabakverarbeitung }\end{array}$ \\
\hline 5 & Erzeugung von Textilien und Textilwaren \\
\hline 6 & $\begin{array}{l}\text { Erzeugung von Bekleidung und Bettwaren, Erzeugung und Reparatur von } \\
\text { Schuhen }\end{array}$ \\
\hline 7 & Erzeugung und Verarbeitung von Leder und Lederersatzstoffen \\
\hline 8 & $\begin{array}{l}\text { Holzplattenerzeugung, Bearbeitung von Holz, Verarbeitung von Holz, Erzeugung } \\
\text { von Musikinstrumenten, Sportartikeln und Spielwaren }\end{array}$ \\
\hline 9 & Erzeugung und Verarbeitung von Papier und Pappe \\
\hline 10 & Druckerei und Vervielfältigung, Verlagswesen \\
\hline 11 & $\begin{array}{l}\text { Erzeugung von Waren aus Gummi und Kunststoffen, Erzeugung von Chemikalien } \\
\text { und chemischen Produkten, Verarbeitung von Erdöl, Erdgas und Kohle }\end{array}$ \\
\hline 12 & $\begin{array}{l}\text { Erzeugung von Waren aus Steinen und Erden, Erzeugung und Bearbeitung von } \\
\text { Glas }\end{array}$ \\
\hline 13 & $\begin{array}{l}\text { Erzeugung von Eisen und NE-Metallen, Stahl- und Leichtmetallbau, Bearbeitung } \\
\text { von Metallen, Erzeugung von Metallwaren, Erzeugung von Maschinen, Erzeugung } \\
\text { von elektrotechnischen Einrichtungen, Erzeugung von Transportmitteln, Erzeu- } \\
\text { gung von feinmechanischen Geräten, Uhren und Schmuckwaren }\end{array}$ \\
\hline 14 & Hoch- und Tiefbau, Ausbau- und Bauhilfsgewerbe, Bauinstallation \\
\hline 15 & Groflhandel, Einzelhandel, Lagerung und Aufbewahrung \\
\hline 16 & Beherbergungs- und Gaststättenwesen \\
\hline 17 & $\begin{array}{l}\text { Straflenverkehr, Eisenbahn- und Seilbahnverkehr, Schiffahrt, Luftverkehr, Trans- } \\
\text { port in Rohrleitungen, Spedition, Nachrichten,bermittlung }\end{array}$ \\
\hline 18 & Geld- und Kreditwesen, Privatversicherung \\
\hline 19 & Realitätenwesen, Rechts- und Wirtschaftsdienste \\
\hline 20 & Körperpflege und Reinigung, Bestattungswesen \\
\hline 21 & Kunst, Unterhaltung, Sport \\
\hline 22 & Gesundheits- und Fürsorgewesen \\
\hline 23 & Unterrichts- und Forschungswesen \\
\hline 24 & $\begin{array}{l}\text { Sozialversicherungsträger, Interessenvertretungen, Einrichtungen der Gebi- } \\
\text { etskörperschaften }\end{array}$ \\
\hline 25 & Haushaltung und Hauswartung \\
\hline
\end{tabular}


Table 11: Codelist for the variable $w i k l \_59$

\begin{tabular}{|c|c|}
\hline code & industry \\
\hline 1 & Landwirtschaft, Fischere \\
\hline 2 & Forstwirtschaft, Jagd \\
\hline 11 & Elektrizitätsversorgung \\
\hline 12 & Gasversorgung \\
\hline 13 & Wärmeversorgung \\
\hline 14 & Wasserversorgung \\
\hline 21 & Kohlenbergbau \\
\hline 22 & Erzbergbau \\
\hline 23 & Erdöl- und Erdgasbergbau \\
\hline 24 & Salzbergbau \\
\hline 25 & Magnesitbergbau \\
\hline 26 & Sonstiger Bergbau, Torfabbau \\
\hline 27 & Steine(Erden)gewinnung \\
\hline 31 & Erzeugung von Nahrungs- und Genuflmitteln \\
\hline 32 & Erzeugung von Getränken, Tabakverarbeitung \\
\hline 33 & Erzeugung von Textilien und Textilwaren \\
\hline 34 & Erzeugung von Bekleidung und Bettwaren \\
\hline 35 & Erzeugung und Reparatur von Schuhen \\
\hline 36 & Erzeugung und Verarbeitung von Leder und Lederersatzstoffen \\
\hline 37 & Holzplattenerzeugung, Bearbeitung von Holz \\
\hline 38 & Verarbeitung von Holz \\
\hline 39 & Erzeugung von Musikinstrumenten, Sportartikeln und Spielwaren \\
\hline 41 & Erzeugung und Verarbeitung von Papier und Pappe \\
\hline 42 & Druckerei und Vervielfältigung \\
\hline 43 & Verlagswesen \\
\hline 44 & Erzeugung von Waren aus Gummi und Kunststoffen \\
\hline 45 & Erzeugung von Chemikalien und chemischen Produkten \\
\hline 46 & Verarbeitung von Erdöl, Erdgas und Kohle \\
\hline 47 & Erzeugung von Waren aus Steinen und Erden \\
\hline 48 & Erzeugung und Bearbeitung von Glas \\
\hline 51 & Erzeugung von Eisen und NE-Metallen \\
\hline 52 & Stahl- und Leichtmetallbau, Bearbeitung von Metallen \\
\hline 53 & Erzeugung von Metallwaren \\
\hline 54 & Erzeugung von Maschinen \\
\hline 56 & Erzeugung von elektrotechnischen Einrichtungen \\
\hline 58 & Erzeugung von Transportmitteln \\
\hline 59 & Erzeugung von feinmechanischen Geräten, Uhren und Schmuckwaren \\
\hline 61 & Hoch- und Tiefbau \\
\hline 62 & Ausbau- und Bauhilfsgewerbe \\
\hline 63 & Bauinstallation \\
\hline 71 & Grosshandel \\
\hline 74 & Einzelhandel \\
\hline 77 & Lagerung und Aufbewahrung \\
\hline 78 & Beherbergungs- und Gaststättenwesen \\
\hline 81 & Straflenverkehr \\
\hline
\end{tabular}

Continued on next page... 


\begin{tabular}{ll}
$\ldots$ table & 11 continued \\
\hline \hline code & industry \\
\hline 82 & Eisenbahn- und Seilbahnverkehr \\
83 & Schiffahrt \\
84 & Luftverkehr \\
85 & Transport in Rohrleitungen, Spedition \\
88 & Nachrichtenübermittlung \\
91 & Geld- und Kreditwesen \\
92 & Privatversicherung \\
93 & Realitätenwesen, Rechts- und Wirtschaftsdienste \\
94 & Körperpflege und Reinigung, Bestattungswesen \\
95 & Kunst, Unterhaltung, Sport \\
96 & Gesundheits- und Fürsorgewesen \\
97 & Unterrichts- und Forschungswesen \\
98 & Sozialversicherungsträger, Interessenvertretungen, Einrichtungen der Gebi- \\
& etskörperschaften \\
99 & Haushaltung und Hauswartung
\end{tabular}


Table 12: Codelist for the variable onace

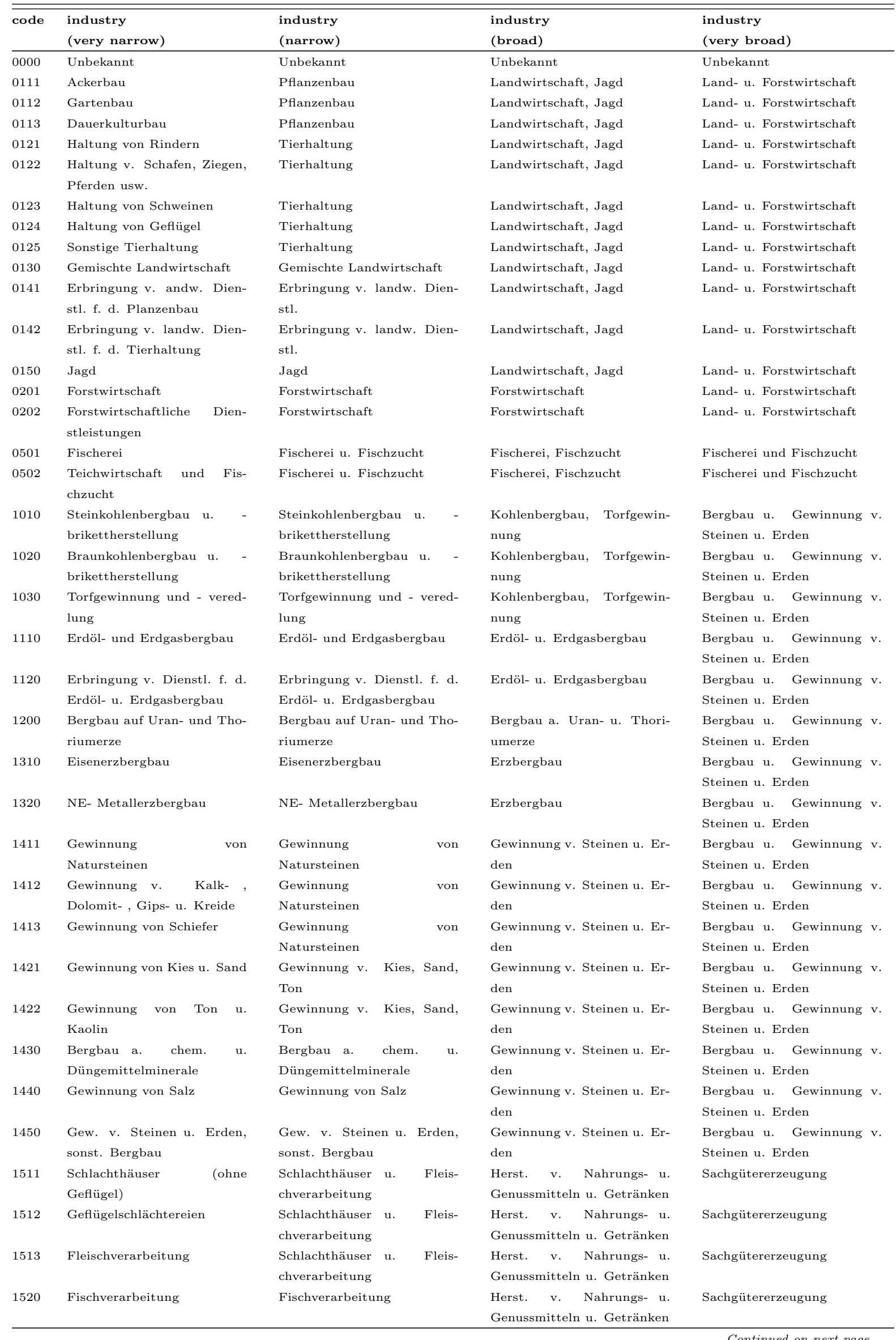

Continued on next page... 


\begin{tabular}{|c|c|c|c|c|}
\hline code & $\begin{array}{l}\text { industry } \\
\text { (very narrow) }\end{array}$ & $\begin{array}{l}\text { industry } \\
\text { (narrow) }\end{array}$ & $\begin{array}{l}\text { industry } \\
\text { (broad) }\end{array}$ & $\begin{array}{l}\text { industry } \\
\text { (very broad) }\end{array}$ \\
\hline 1531 & Verarbeitung von Kartoffeln & $\begin{array}{l}\text { Obst- } \\
\text { Gemüseverarbeitung }\end{array}$ & $\begin{array}{l}\text { Herst. v. Nahrungs- u. } \\
\text { Genussmitteln u. Getränken }\end{array}$ & Sachgütererzeugung \\
\hline 1532 & $\begin{array}{l}\text { Herstellung von Frucht- und } \\
\text { Gemüsesäften }\end{array}$ & $\begin{array}{l}\text { Obst- } \\
\text { Gemüseverarbeitung }\end{array}$ & $\begin{array}{l}\text { Herst. v. Nahrungs- u. } \\
\text { Genussmitteln u. Getränken }\end{array}$ & Sachgütererzeugung \\
\hline 1533 & $\begin{array}{l}\text { Verarbeitung von Obst und } \\
\text { Gemüse a. n. g. }\end{array}$ & $\begin{array}{l}\text { Obst- } \\
\text { Gemüseverarbeitung }\end{array}$ & $\begin{array}{l}\text { Herst. v. Nahrungs- u. } \\
\text { Genussmitteln u. Getränken }\end{array}$ & Sachgütererzeugung \\
\hline 1541 & $\begin{array}{l}\text { Herst. v. rohen Ölen u. Fet- } \\
\text { ten }\end{array}$ & $\begin{array}{l}\text { Herst. v. pflanzl. u. tier. } \\
\text { Ölen u. Fetten }\end{array}$ & $\begin{array}{l}\text { Herst. v. Nahrungs- u. } \\
\text { Genussmitteln u. Getränken }\end{array}$ & Sachgütererzeugung \\
\hline 1542 & $\begin{array}{l}\text { Herst. v. raffinierten Ölen u. } \\
\text { Fette }\end{array}$ & $\begin{array}{l}\text { Herst. v. pflanzl. u. tier. } \\
\text { Ölen u. Fetten }\end{array}$ & $\begin{array}{l}\text { Herst. v. Nahrungs- u. } \\
\text { Genussmitteln u. Getränken }\end{array}$ & Sachgütererzeugung \\
\hline 1543 & $\begin{array}{l}\text { Herst. v. Margarine u. ä. } \\
\text { Nahrungsfetten }\end{array}$ & $\begin{array}{l}\text { Herst. v. pflanzl. u. tier. } \\
\text { Ölen u. Fetten }\end{array}$ & $\begin{array}{l}\text { Herst. v. Nahrungs- u. } \\
\text { Genussmitteln u. Getränken }\end{array}$ & Sachgütererzeugung \\
\hline 1551 & Milchverarbeitung & Milchverarbeitung & $\begin{array}{l}\text { Herst. v. Nahrungs- u. } \\
\text { Genussmitteln u. Getränken }\end{array}$ & Sachgütererzeugung \\
\hline 1552 & Herstellung von Speiseeis & Milchverarbeitung & $\begin{array}{l}\text { Herst. v. Nahrungs- u. } \\
\text { Genussmitteln u. Getränken }\end{array}$ & Sachgütererzeugung \\
\hline 1561 & Mahl- und Schälmühlen & $\begin{array}{l}\text { Mahl- u. Schälmühlen, } \\
\text { Herst. v. Stärke }\end{array}$ & $\begin{array}{l}\text { Herst. v. Nahrungs- u. } \\
\text { Genussmitteln u. Getränken }\end{array}$ & Sachgütererzeugung \\
\hline 1562 & $\begin{array}{l}\text { Herst. v. Stärke u. } \\
\text { Stärkeerzeugniss }\end{array}$ & $\begin{array}{l}\text { Mahl- u. Schälmühlen, } \\
\text { Herst. v. Stärke }\end{array}$ & $\begin{array}{l}\text { Herst. v. Nahrungs- u. } \\
\text { Genussmitteln u. Getränken }\end{array}$ & Sachgütererzeugung \\
\hline 1571 & $\begin{array}{l}\text { Herst. v. Futtermitteln für } \\
\text { Nutztiere }\end{array}$ & Herst. v. Futtermitteln & $\begin{array}{l}\text { Herst. v. Nahrungs- u. } \\
\text { Genussmitteln u. Getränken }\end{array}$ & Sachgütererzeugung \\
\hline 1572 & $\begin{array}{l}\text { Herst. v. Futtermitteln f. } \\
\text { sonst. Tiere }\end{array}$ & Herst. v. Futtermitteln & $\begin{array}{l}\text { Herst. v. Nahrungs- u. } \\
\text { Genussmitteln u. Getränken }\end{array}$ & Sachgütererzeugung \\
\hline 1581 & $\begin{array}{l}\text { Herst. v. Backwaren (o. } \\
\text { Dauerbackwaren) }\end{array}$ & $\begin{array}{l}\text { Herst. v. stonst. Nahrungs- } \\
\text { u. Genußmitteln }\end{array}$ & $\begin{array}{l}\text { Herst. v. Nahrungs- u. } \\
\text { Genussmitteln u. Getränken }\end{array}$ & Sachgütererzeugung \\
\hline 1582 & Herst. v. Dauerbackwaren & $\begin{array}{l}\text { Herst. v. stonst. Nahrungs- } \\
\text { u. Genußmitteln }\end{array}$ & $\begin{array}{l}\text { Herst. v. Nahrungs- u. } \\
\text { Genussmitteln u. Getränken }\end{array}$ & Sachgütererzeugung \\
\hline 1583 & Herst. v. Zucker & $\begin{array}{l}\text { Herst. v. stonst. Nahrungs- } \\
\text { u. Genußmitteln }\end{array}$ & $\begin{array}{l}\text { Herst. v. Nahrungs- u. } \\
\text { Genussmitteln u. Getränken }\end{array}$ & Sachgütererzeugung \\
\hline 1584 & $\begin{array}{l}\text { Herst. v. Süßwaren (o. } \\
\text { Dauerbackwaren) }\end{array}$ & $\begin{array}{l}\text { Herst. v. stonst. Nahrungs- } \\
\text { u. Genußmitteln }\end{array}$ & $\begin{array}{l}\text { Herst. v. Nahrungs- u. } \\
\text { Genussmitteln u. Getränken }\end{array}$ & Sachgütererzeugung \\
\hline 1585 & Herstellung von Teigwaren & $\begin{array}{l}\text { Herst. v. stonst. Nahrungs- } \\
\text { u. Genußmitteln }\end{array}$ & $\begin{array}{l}\text { Herst. v. Nahrungs- u. } \\
\text { Genussmitteln u. Getränken }\end{array}$ & Sachgütererzeugung \\
\hline 1586 & $\begin{array}{l}\text { Verarbeitung v. Kaffee u. } \\
\text { Tee }\end{array}$ & $\begin{array}{l}\text { Herst. v. stonst. Nahrungs- } \\
\text { u. Genußmitteln }\end{array}$ & $\begin{array}{l}\text { Herst. v. Nahrungs- u. } \\
\text { Genussmitteln u. Getränken }\end{array}$ & Sachgütererzeugung \\
\hline 1587 & $\begin{array}{l}\text { Herstellung von Würzen und } \\
\text { Soßen }\end{array}$ & $\begin{array}{l}\text { Herst. v. stonst. Nahrungs- } \\
\text { u. Genußmitteln }\end{array}$ & $\begin{array}{l}\text { Herst. v. Nahrungs- u. } \\
\text { Genussmitteln u. Getränken }\end{array}$ & Sachgütererzeugung \\
\hline 1588 & $\begin{array}{l}\text { Herst. v. homogenisierten u. } \\
\text { diätetischen Nahrungsm. }\end{array}$ & $\begin{array}{l}\text { Herst. v. stonst. Nahrungs- } \\
\text { u. Genußmitteln }\end{array}$ & $\begin{array}{l}\text { Herst. v. Nahrungs- u. } \\
\text { Genussmitteln u. Getränken }\end{array}$ & Sachgütererzeugung \\
\hline 1589 & $\begin{array}{l}\text { Herst. v. sonst. Nahrungs- } \\
\text { u. Genußm. }\end{array}$ & $\begin{array}{l}\text { Herst. v. stonst. Nahrungs- } \\
\text { u. Genußmitteln }\end{array}$ & $\begin{array}{l}\text { Herst. v. Nahrungs- u. } \\
\text { Genussmitteln u. Getränken }\end{array}$ & Sachgütererzeugung \\
\hline 1591 & Herstellung von Spirituosen & Getränkeherstellung & $\begin{array}{l}\text { Herst. v. Nahrungs- u. } \\
\text { Genussmitteln u. Getränken }\end{array}$ & Sachgütererzeugung \\
\hline 1592 & Alkoholbrennerei & Getränkeherstellung & $\begin{array}{l}\text { Herst. v. Nahrungs- u. } \\
\text { Genussmitteln u. Getränken }\end{array}$ & Sachgütererzeugung \\
\hline 1593 & Herstellung von Wein & Getränkeherstellung & $\begin{array}{l}\text { Herst. v. Nahrungs- u. } \\
\text { Genussmitteln u. Getränken }\end{array}$ & Sachgütererzeugung \\
\hline 1594 & $\begin{array}{l}\text { Herst. v. Apfelwein u. } \\
\text { sonst. Fruchtweinen }\end{array}$ & Getränkeherstellung & $\begin{array}{l}\text { Herst. v. Nahrungs- u. } \\
\text { Genussmitteln u. Getränken }\end{array}$ & Sachgütererzeugung \\
\hline 1595 & $\begin{array}{l}\text { Herst. v. Wermut u. sonst. } \\
\text { aromat. Weinen }\end{array}$ & Getränkeherstellung & $\begin{array}{l}\text { Herst. v. Nahrungs- u. } \\
\text { Genussmitteln u. Getränken }\end{array}$ & Sachgütererzeugung \\
\hline 1596 & Herstellung von Bier & Getränkeherstellung & $\begin{array}{l}\text { Herst. v. Nahrungs- u. } \\
\text { Genussmitteln u. Getränken }\end{array}$ & Sachgütererzeugung \\
\hline 1597 & Herstellung von Malz & Getränkeherstellung & $\begin{array}{l}\text { Herst. v. Nahrungs- u. } \\
\text { Genussmitteln u. Getränken }\end{array}$ & Sachgütererzeugung \\
\hline 1598 & $\begin{array}{l}\text { Gewinnung u. Abfüllung } \\
\text { natürl. Mineralwässer }\end{array}$ & Getränkeherstellung & $\begin{array}{l}\text { Herst. v. Nahrungs- u. } \\
\text { Genussmitteln u. Getränken }\end{array}$ & Sachgütererzeugung \\
\hline 1600 & Tabakverarbeitung & Tabakverarbeitung & Tabakverarbeitung & Sachgütererzeugung \\
\hline 1711 & $\begin{array}{l}\text { Baumwollaufbereitung } \\
\text { Spinnerei }\end{array}$ & $\begin{array}{l}\text { Spinnstoffaufber. u. Spin- } \\
\text { nerei }\end{array}$ & $\begin{array}{l}\text { Herst. v. Textilien u. Tex- } \\
\text { tilwaren (o. Bekleidung) }\end{array}$ & Sachgütererzeugung \\
\hline 1712 & $\begin{array}{l}\text { Wollaufbereitung u. Streich- } \\
\text { garnspinnerei }\end{array}$ & $\begin{array}{l}\text { Spinnstoffaufber. u. Spin- } \\
\text { nerei }\end{array}$ & $\begin{array}{l}\text { Herst. v. Textilien u. Tex- } \\
\text { tilwaren (o. Bekleidung) }\end{array}$ & Sachgütererzeugung \\
\hline
\end{tabular}




\begin{tabular}{|c|c|c|c|c|}
\hline code & $\begin{array}{l}\text { industry } \\
\text { (very narrow) }\end{array}$ & $\begin{array}{l}\text { industry } \\
\text { (narrow) }\end{array}$ & $\begin{array}{l}\text { industry } \\
\text { (broad) }\end{array}$ & $\begin{array}{l}\text { industry } \\
\text { (very broad) }\end{array}$ \\
\hline 1713 & $\begin{array}{l}\text { Wollaufbereitung u. Kamm- } \\
\text { garnspinnerei }\end{array}$ & $\begin{array}{l}\text { Spinnstoffaufber. u. Spin- } \\
\text { nerei }\end{array}$ & $\begin{array}{l}\text { Herst. v. Textilien u. Tex- } \\
\text { tilwaren (o. Bekleidung) }\end{array}$ & Sachgütererzeugung \\
\hline 1714 & $\begin{array}{l}\text { Flachsaufbereitung u. Spin- } \\
\text { nerei }\end{array}$ & $\begin{array}{l}\text { Spinnstoffaufber. u. Spin- } \\
\text { nerei }\end{array}$ & $\begin{array}{l}\text { Herst. v. Textilien u. Tex- } \\
\text { tilwaren (o. Bekleidung) }\end{array}$ & Sachgütererzeugung \\
\hline 1715 & $\begin{array}{l}\text { Zwirnen u. Texturieren v. } \\
\text { Filamentgarnen }\end{array}$ & $\begin{array}{l}\text { Spinnstoffaufber. u. Spin- } \\
\text { nerei }\end{array}$ & $\begin{array}{l}\text { Herst. v. Textilien u. Tex- } \\
\text { tilwaren (o. Bekleidung) }\end{array}$ & Sachgütererzeugung \\
\hline 1716 & Nähgarnfertigung & $\begin{array}{l}\text { Spinnstoffaufber. u. Spin- } \\
\text { nerei }\end{array}$ & $\begin{array}{l}\text { Herst. v. Textilien u. Tex- } \\
\text { tilwaren (o. Bekleidung) }\end{array}$ & Sachgütererzeugung \\
\hline 1717 & $\begin{array}{l}\text { Sonst. Spinnstoffaufbere- } \\
\text { itung u. Spinnerei }\end{array}$ & $\begin{array}{l}\text { Spinnstoffaufber. u. Spin- } \\
\text { nerei }\end{array}$ & $\begin{array}{l}\text { Herst. v. Textilien u. Tex- } \\
\text { tilwaren (o. Bekleidung) }\end{array}$ & Sachgütererzeugung \\
\hline 1721 & Baumwollweberei & Weberei & $\begin{array}{l}\text { Herst. v. Textilien u. Tex- } \\
\text { tilwaren (o. Bekleidung) }\end{array}$ & Sachgütererzeugung \\
\hline 1722 & Streichgarnweberei & Weberei & $\begin{array}{l}\text { Herst. v. Textilien u. Tex- } \\
\text { tilwaren (o. Bekleidung) }\end{array}$ & Sachgütererzeugung \\
\hline 1723 & Kammgarnweberei & Weberei & $\begin{array}{l}\text { Herst. v. Textilien u. Tex- } \\
\text { tilwaren (o. Bekleidung) }\end{array}$ & Sachgütererzeugung \\
\hline 1724 & $\begin{array}{l}\text { Seiden- und Filamentgar- } \\
\text { nweberei }\end{array}$ & Weberei & $\begin{array}{l}\text { Herst. v. Textilien u. Tex- } \\
\text { tilwaren (o. Bekleidung) }\end{array}$ & Sachgütererzeugung \\
\hline 1725 & Sonstige Weberei & Weberei & $\begin{array}{l}\text { Herst. v. Textilien u. Tex- } \\
\text { tilwaren (o. Bekleidung) }\end{array}$ & Sachgütererzeugung \\
\hline 1730 & Textilveredlung & Textilveredlung & $\begin{array}{l}\text { Herst. v. Textilien u. Tex- } \\
\text { tilwaren (o. Bekleidung) }\end{array}$ & Sachgütererzeugung \\
\hline 1740 & $\begin{array}{l}\text { Herst. v. konfektionierten } \\
\text { Textilwaren }\end{array}$ & $\begin{array}{l}\text { Herst. v. konfektionierten } \\
\text { Textilwaren }\end{array}$ & $\begin{array}{l}\text { Herst. v. Textilien u. Tex- } \\
\text { tilwaren (o. Bekleidung) }\end{array}$ & Sachgütererzeugung \\
\hline 1751 & Herst. von Teppichen & $\begin{array}{l}\text { Herst. v. stonst. Textil- } \\
\text { waren }\end{array}$ & $\begin{array}{l}\text { Herst. v. Textilien u. Tex- } \\
\text { tilwaren (o. Bekleidung) }\end{array}$ & Sachgütererzeugung \\
\hline 1752 & Herst. von Seilerwaren & $\begin{array}{l}\text { Herst. v. stonst. Textil- } \\
\text { waren }\end{array}$ & $\begin{array}{l}\text { Herst. v. Textilien u. Tex- } \\
\text { tilwaren (o. Bekleidung) }\end{array}$ & Sachgütererzeugung \\
\hline 1753 & $\begin{array}{l}\text { Herst. v. Vliesstoff u. } \\
\text { Erzeugnissen daraus }\end{array}$ & $\begin{array}{l}\text { Herst. v. stonst. Textil- } \\
\text { waren }\end{array}$ & $\begin{array}{l}\text { Herst. v. Textilien u. Tex- } \\
\text { tilwaren (o. Bekleidung) }\end{array}$ & Sachgütererzeugung \\
\hline 1754 & $\begin{array}{l}\text { Herst. v. Stickereien u. v. } \\
\text { sonst. Textilwaren }\end{array}$ & $\begin{array}{l}\text { Herst. v. stonst. Textil- } \\
\text { waren }\end{array}$ & $\begin{array}{l}\text { Herst. v. Textilien u. Tex- } \\
\text { tilwaren (o. Bekleidung) }\end{array}$ & Sachgütererzeugung \\
\hline 1760 & $\begin{array}{l}\text { Herst. v. gewirktem u. } \\
\text { gestricktem Stoff }\end{array}$ & $\begin{array}{l}\text { Herst. v. gewirktem u. } \\
\text { gestricktem Stoff }\end{array}$ & $\begin{array}{l}\text { Herst. v. Textilien u. Tex- } \\
\text { tilwaren (o. Bekleidung) }\end{array}$ & Sachgütererzeugung \\
\hline 1771 & $\begin{array}{l}\text { Herstellung } \\
\text { Strumpfwaren }\end{array}$ & $\begin{array}{l}\text { Herst. v. gewirktem u. } \\
\text { gestrickten Erzeugn. }\end{array}$ & $\begin{array}{l}\text { Herst. v. Textilien u. Tex- } \\
\text { tilwaren (o. Bekleidung) }\end{array}$ & Sachgütererzeugung \\
\hline 1772 & $\begin{array}{l}\text { Herstellung von Pullovern, } \\
\text { Strickjacken u. ä }\end{array}$ & $\begin{array}{l}\text { Herst. v. gewirktem u. } \\
\text { gestrickten Erzeugn. }\end{array}$ & $\begin{array}{l}\text { Herst. v. Textilien u. Tex- } \\
\text { tilwaren (o. Bekleidung) }\end{array}$ & Sachgütererzeugung \\
\hline 1810 & $\begin{array}{l}\text { Herstellung von Lederbeklei- } \\
\text { dung }\end{array}$ & $\begin{array}{l}\text { Herstellung von Lederbeklei- } \\
\text { dung }\end{array}$ & Herstellung von Bekleidung & Sachgütererzeugung \\
\hline 1821 & $\begin{array}{l}\text { Herst. v. Arbeits- u. Berufs- } \\
\text { bekleidung }\end{array}$ & $\begin{array}{l}\text { Herst. } \quad \text { v. } \quad \text { Bekleidung(o. } \\
\text { Leder) }\end{array}$ & Herstellung von Bekleidung & Sachgütererzeugung \\
\hline 1822 & $\begin{array}{l}\text { Herst. v. sonst. Oberbeklei- } \\
\text { dung }\end{array}$ & $\begin{array}{l}\text { Herst. } \quad \text { v. } \quad \text { Bekleidung(o. } \\
\text { Leder) }\end{array}$ & Herstellung von Bekleidung & Sachgütererzeugung \\
\hline 1823 & Herstellung von Wäsche & $\begin{array}{l}\text { Herst. } \quad \text { v. } \quad \text { Bekleidung(o. } \\
\text { Leder) }\end{array}$ & Herstellung von Bekleidung & Sachgütererzeugung \\
\hline 1824 & $\begin{array}{l}\text { Herst. v. sonst. Bekleidung } \\
\text { u. Zubehör }\end{array}$ & $\begin{array}{l}\text { Herst. } \quad \text { v. } \quad \text { Bekleidung(o. } \\
\text { Leder })\end{array}$ & Herstellung von Bekleidung & Sachgütererzeugung \\
\hline 1830 & $\begin{array}{l}\text { Zurichtung u. Färben v. } \\
\text { Fellen, Herst. v. Pelzw. }\end{array}$ & $\begin{array}{l}\text { Zurichtung u. Färben v. } \\
\text { Fellen, Herst. v. Pelzw. }\end{array}$ & Herstellung von Bekleidung & Sachgütererzeugung \\
\hline 1910 & Ledererzeugung & Ledererzeugung & $\begin{array}{l}\text { Ledererzeugung u. - verar- } \\
\text { beitung }\end{array}$ & Sachgütererzeugung \\
\hline 1920 & $\begin{array}{l}\text { Lederverarbeitung (o. Bek- } \\
\text { leidung) }\end{array}$ & $\begin{array}{l}\text { Lederverarbeitung (o. Bek- } \\
\text { leidung) }\end{array}$ & $\begin{array}{l}\text { Ledererzeugung u. - verar- } \\
\text { beitung }\end{array}$ & Sachgütererzeugung \\
\hline 1930 & Herstellung von Schuhen & Herstellung von Schuhen & $\begin{array}{l}\text { Ledererzeugung } \mathrm{u} \text {. - verar- } \\
\text { beitung }\end{array}$ & Sachgütererzeugung \\
\hline 2010 & $\begin{array}{l}\text { Säge- , Hobel- u. Holz- } \\
\text { imprägnierwerke }\end{array}$ & $\begin{array}{l}\text { Säge- , Hobel- u. Holz- } \\
\text { imprägnierwerke }\end{array}$ & $\begin{array}{l}\text { Be- u. Verarbeitung v. Holz } \\
\text { (o. Möbel) }\end{array}$ & Sachgütererzeugung \\
\hline 2020 & $\begin{array}{ll}\text { Furnier- , Sperrholz- , } & \text {, } \\
\text { Holzfaser- u. Holzspanplat- } \\
\text { tenwerke }\end{array}$ & $\begin{array}{l}\text { Furnier- , Sperrholz- , } \\
\text { Holzfaser- u. Holzspanplat- } \\
\text { ten }\end{array}$ & $\begin{array}{l}\text { Be- u. Verarbeitung v. Holz } \\
\text { (o. Möbel) }\end{array}$ & Sachgütererzeugung \\
\hline 2030 & $\begin{array}{l}\text { Herst. v. Konstruktion- } \\
\text { steilen, Fertigbauteilen }\end{array}$ & $\begin{array}{l}\text { Herst. v. Konstruktion- } \\
\text { steilen, Fertigbauteilen }\end{array}$ & $\begin{array}{l}\text { Be- u. Verarbeitung v. Holz } \\
\text { (o. Möbel) }\end{array}$ & Sachgütererzeugung \\
\hline
\end{tabular}









\begin{tabular}{|c|c|c|c|c|}
\hline code & $\begin{array}{l}\text { industry } \\
\text { (very narrow) }\end{array}$ & $\begin{array}{l}\text { industry } \\
\text { (narrow) }\end{array}$ & $\begin{array}{l}\text { industry } \\
\text { (broad) }\end{array}$ & $\begin{array}{l}\text { industry } \\
\text { (very broad) }\end{array}$ \\
\hline 2330 & $\begin{array}{l}\text { Herst. u. Verarbeitung v. } \\
\text { Spalt- u. Brutstoffen }\end{array}$ & $\begin{array}{l}\text { Herst. u. Verarbeitung v. } \\
\text { Spalt- u. Brutstoffen }\end{array}$ & $\begin{array}{l}\text { Kokerei, } \\
\text { eralölverarbeitung }\end{array}$ & Sachgütererzeugung \\
\hline 2411 & Herst. von Industriegasen & $\begin{array}{l}\text { Herst. v. chem. Grundstof- } \\
\text { fen }\end{array}$ & $\begin{array}{l}\text { Herst. v. chemik. u. chem. } \\
\text { Erzeugnissen }\end{array}$ & Sachgütererzeugung \\
\hline 2412 & $\begin{array}{l}\text { Herst. v. Farbstoffen u. Pig- } \\
\text { menten }\end{array}$ & $\begin{array}{l}\text { Herst. v. chem. Grundstof- } \\
\text { fen }\end{array}$ & $\begin{array}{l}\text { Herst. v. chemik. u. chem. } \\
\text { Erzeugnissen }\end{array}$ & Sachgütererzeugung \\
\hline 2413 & $\begin{array}{l}\text { Herst. v. sonst. anorganis- } \\
\text { chen Grundstoffen }\end{array}$ & $\begin{array}{l}\text { Herst. v. chem. Grundstof- } \\
\text { fen }\end{array}$ & $\begin{array}{l}\text { Herst. v. chemik. u. chem. } \\
\text { Erzeugnissen }\end{array}$ & Sachgütererzeugung \\
\hline 2414 & $\begin{array}{l}\text { Herst. v. sonst. organischen } \\
\text { Grundstoffen }\end{array}$ & $\begin{array}{l}\text { Herst. v. chem. Grundstof- } \\
\text { fen }\end{array}$ & $\begin{array}{l}\text { Herst. v. chemik. u. chem. } \\
\text { Erzeugnissen }\end{array}$ & Sachgütererzeugung \\
\hline 2415 & $\begin{array}{l}\text { Herst. v. Düngemitteln u. } \\
\text { Stickstoffverb. }\end{array}$ & $\begin{array}{l}\text { Herst. v. chem. Grundstof- } \\
\text { fen }\end{array}$ & $\begin{array}{l}\text { Herst. v. chemik. u. chem. } \\
\text { Erzeugnissen }\end{array}$ & Sachgütererzeugung \\
\hline 2416 & $\begin{array}{l}\text { Herst. v. Kunststoff in } \\
\text { Primärformen }\end{array}$ & $\begin{array}{l}\text { Herst. v. chem. Grundstof- } \\
\text { fen }\end{array}$ & $\begin{array}{l}\text { Herst. v. chemik. u. chem. } \\
\text { Erzeugnissen }\end{array}$ & Sachgütererzeugung \\
\hline 2417 & $\begin{array}{l}\text { Herst. v. synthet. } \\
\text { Kautschuk in Primärform }\end{array}$ & $\begin{array}{l}\text { Herst. v. chem. Grundstof- } \\
\text { fen }\end{array}$ & $\begin{array}{l}\text { Herst. v. chemik. u. chem. } \\
\text { Erzeugnissen }\end{array}$ & Sachgütererzeugung \\
\hline 2420 & $\begin{array}{l}\text { Herst. } \\
\text { Schädlingsbekämpfungs- } \\
\text { u. Pfl. - schutz }\end{array}$ & $\begin{array}{l}\text { Herst. } \\
\text { Schädlingsbekämpfungs- } \\
\text { u. Pfl. - schutz }\end{array}$ & $\begin{array}{l}\text { Herst. v. chemik. u. chem. } \\
\text { Erzeugnissen }\end{array}$ & Sachgütererzeugung \\
\hline 2430 & $\begin{array}{l}\text { Herst. v. Anstrichmitteln, } \\
\text { Druckfarbe u. Kitten }\end{array}$ & $\begin{array}{l}\text { Herst. v. Anstrichmitteln, } \\
\text { Druckfarbe u. Kitten }\end{array}$ & $\begin{array}{l}\text { Herst. v. chemik. u. chem. } \\
\text { Erzeugnissen }\end{array}$ & Sachgütererzeugung \\
\hline 2441 & $\begin{array}{l}\text { Herst. v. pharmaz. Grund- } \\
\text { stoffen }\end{array}$ & $\begin{array}{l}\text { Herst. v. pharmaz. Erzeug- } \\
\text { nissen }\end{array}$ & $\begin{array}{l}\text { Herst. v. chemik. u. chem. } \\
\text { Erzeugnissen }\end{array}$ & Sachgütererzeugung \\
\hline 2442 & $\begin{array}{l}\text { Herst. v. pharmaz. } \\
\text { Spezialitäten }\end{array}$ & $\begin{array}{l}\text { Herst. v. pharmaz. Erzeug- } \\
\text { nissen }\end{array}$ & $\begin{array}{l}\text { Herst. v. chemik. u. chem. } \\
\text { Erzeugnissen }\end{array}$ & Sachgütererzeugung \\
\hline 2451 & $\begin{array}{l}\text { Herst. v. Seifen, Wasch- , } \\
\text { Reinigungsmitteln }\end{array}$ & $\begin{array}{l}\text { Herst. v. Seifen, Wasch- u. } \\
\text { Körperpflegemitteln }\end{array}$ & $\begin{array}{l}\text { Herst. v. chemik. u. chem. } \\
\text { Erzeugnissen }\end{array}$ & Sachgütererzeugung \\
\hline 2452 & $\begin{array}{l}\text { Herst. v. Duft- u. } \\
\text { Körperpflegemitteln }\end{array}$ & $\begin{array}{l}\text { Herst. v. Seifen, Wasch- u. } \\
\text { Körperpflegemitteln }\end{array}$ & $\begin{array}{l}\text { Herst. v. chemik. u. chem. } \\
\text { Erzeugnissen }\end{array}$ & Sachgütererzeugung \\
\hline 2461 & $\begin{array}{l}\text { Herst. v. pyrotechnischen } \\
\text { Erzeugnissen }\end{array}$ & $\begin{array}{l}\text { Herst. v. sonst. chem. } \\
\text { Erzeugnissen }\end{array}$ & $\begin{array}{l}\text { Herst. v. chemik. u. chem. } \\
\text { Erzeugnissen }\end{array}$ & Sachgütererzeugung \\
\hline 2462 & $\begin{array}{l}\text { Herst. v. Klebstoffen und } \\
\text { Gelatine }\end{array}$ & $\begin{array}{l}\text { Herst. v. sonst. chem. } \\
\text { Erzeugnissen }\end{array}$ & $\begin{array}{l}\text { Herst. v. chemik. u. chem. } \\
\text { Erzeugnissen }\end{array}$ & Sachgütererzeugung \\
\hline 2463 & Herst. v. ätherischen Ölen & $\begin{array}{l}\text { Herst. v. sonst. chem. } \\
\text { Erzeugnissen }\end{array}$ & $\begin{array}{l}\text { Herst. v. chemik. u. chem. } \\
\text { Erzeugnissen }\end{array}$ & Sachgütererzeugung \\
\hline 2464 & $\begin{array}{l}\text { Herst. v. fotochemischen } \\
\text { Erzeugnissen }\end{array}$ & $\begin{array}{l}\text { Herst. v. sonst. chem. } \\
\text { Erzeugnissen }\end{array}$ & $\begin{array}{l}\text { Herst. v. chemik. u. chem. } \\
\text { Erzeugnissen }\end{array}$ & Sachgütererzeugung \\
\hline 2465 & $\begin{array}{l}\text { Herst. v. unbesp. Ton- } \\
\text { Bild- u. Datenträgern }\end{array}$ & $\begin{array}{l}\text { Herst. v. sonst. chem. } \\
\text { Erzeugnissen }\end{array}$ & $\begin{array}{l}\text { Herst. v. chemik. u. chem. } \\
\text { Erzeugnissen }\end{array}$ & Sachgütererzeugung \\
\hline 2466 & $\begin{array}{l}\text { Herst. v. sonst. chem. } \\
\text { Erzeugnissen }\end{array}$ & $\begin{array}{l}\text { Herst. v. sonst. chem. } \\
\text { Erzeugnissen }\end{array}$ & $\begin{array}{l}\text { Herst. v. chemik. u. chem. } \\
\text { Erzeugnissen }\end{array}$ & Sachgütererzeugung \\
\hline 2470 & $\begin{array}{l}\text { Herstellung } \\
\text { Chemiefasern }\end{array}$ & $\begin{array}{l}\text { Herstellung } \\
\text { Chemiefasern }\end{array}$ & $\begin{array}{l}\text { Herst. v. chemik. u. chem. } \\
\text { Erzeugnissen }\end{array}$ & Sachgütererzeugung \\
\hline 2511 & Herstellung von Bereifungen & $\begin{array}{l}\text { Herstellung von Gummi- } \\
\text { waren }\end{array}$ & $\begin{array}{l}\text { Herst. v. Gummi- u. Kunst- } \\
\text { stoffwaren }\end{array}$ & Sachgütererzeugung \\
\hline 2512 & $\begin{array}{l}\text { Runderneuerung von Berei- } \\
\text { fungen }\end{array}$ & $\begin{array}{l}\text { Herstellung von Gummi- } \\
\text { waren }\end{array}$ & $\begin{array}{l}\text { Herst. v. Gummi- u. Kunst- } \\
\text { stoffwaren }\end{array}$ & Sachgütererzeugung \\
\hline 2513 & $\begin{array}{l}\text { Herstellung von sonstigen } \\
\text { Gummiwaren }\end{array}$ & $\begin{array}{l}\text { Herstellung von Gummi- } \\
\text { waren }\end{array}$ & $\begin{array}{l}\text { Herst. v. Gummi- u. Kunst- } \\
\text { stoffwaren }\end{array}$ & Sachgütererzeugung \\
\hline 2521 & $\begin{array}{l}\text { Herst. v. Platten, Folien, } \\
\text { Schläuchen a. Kunststoff }\end{array}$ & $\begin{array}{l}\text { Herstellung von Kunststof- } \\
\text { fwaren }\end{array}$ & $\begin{array}{l}\text { Herst. v. Gummi- u. Kunst- } \\
\text { stoffwaren }\end{array}$ & Sachgütererzeugung \\
\hline 2522 & $\begin{array}{l}\text { Herst. v. Verpackungsmit- } \\
\text { teln a. Kunststoff }\end{array}$ & $\begin{array}{l}\text { Herstellung von Kunststof- } \\
\text { fwaren }\end{array}$ & $\begin{array}{l}\text { Herst. v. Gummi- u. Kunst- } \\
\text { stoffwaren }\end{array}$ & Sachgütererzeugung \\
\hline 2523 & $\begin{array}{l}\text { Herst. v. Baubedarfsar- } \\
\text { tikeln a. Kunststoff }\end{array}$ & $\begin{array}{l}\text { Herstellung von Kunststof- } \\
\text { fwaren }\end{array}$ & $\begin{array}{l}\text { Herst. v. Gummi- u. Kunst- } \\
\text { stoffwaren }\end{array}$ & Sachgütererzeugung \\
\hline 2524 & $\begin{array}{l}\text { Herst. v. sonstigen Kunst- } \\
\text { stoffwaren }\end{array}$ & $\begin{array}{l}\text { Herstellung von Kunststof- } \\
\text { fwaren }\end{array}$ & $\begin{array}{l}\text { Herst. v. Gummi- u. Kunst- } \\
\text { stoffwaren }\end{array}$ & Sachgütererzeugung \\
\hline 2611 & Herstellung von Flachglas & Herst. und Bearb. von Glas & $\begin{array}{l}\text { Herst. u. Bearb. v. Glas } \\
\text { und Stein }\end{array}$ & Sachgütererzeugung \\
\hline 2612 & $\begin{array}{l}\text { Veredlung u. Bearbeitung } \\
\text { von Flachglas }\end{array}$ & Herst. und Bearb. von Glas & $\begin{array}{l}\text { Herst. u. Bearb. v. Glas } \\
\text { und Stein }\end{array}$ & Sachgütererzeugung \\
\hline 2613 & Herstellung von Hohlglas & Herst. und Bearb. von Glas & $\begin{array}{l}\text { Herst. u. Bearb. v. Glas } \\
\text { und Stein }\end{array}$ & Sachgütererzeugung \\
\hline
\end{tabular}




\begin{tabular}{|c|c|c|c|c|c|}
\hline code & $\begin{array}{l}\text { industry } \\
\text { (very narrow) }\end{array}$ & $\begin{array}{l}\text { industry } \\
\text { (narrow) }\end{array}$ & $\begin{array}{l}\text { industry } \\
\text { (broad) }\end{array}$ & & $\begin{array}{l}\text { industry } \\
\text { (very broad) }\end{array}$ \\
\hline 2614 & Herstellung von Glasfaser & Herst. und Bearb. von Glas & $\begin{array}{l}\text { Herst. u. Bearb. } \\
\text { und Stein }\end{array}$ & v. Glas & Sachgütererzeugung \\
\hline 2615 & $\begin{array}{l}\text { Herst. , Veredlung u. Bear- } \\
\text { beitung v. sonst. Glas }\end{array}$ & Herst. und Bearb. von Glas & $\begin{array}{l}\text { Herst. u. Bearb. } \\
\text { und Stein }\end{array}$ & v. Glas & Sachgütererzeugung \\
\hline 2621 & $\begin{array}{l}\text { Herst. v. keramischen } \\
\text { Haushaltswaren }\end{array}$ & $\begin{array}{l}\text { Keramik (o. Ziegelei u. } \\
\text { Baukeramik) }\end{array}$ & $\begin{array}{l}\text { Herst. u. Bearb. } \\
\text { und Stein }\end{array}$ & v. Glas & Sachgütererzeugung \\
\hline 2622 & Herst. von Sanitärkeramik & $\begin{array}{l}\text { Keramik (o. Ziegelei u. } \\
\text { Baukeramik) }\end{array}$ & $\begin{array}{l}\text { Herst. u. Bearb. } \\
\text { und Stein }\end{array}$ & v. Glas & Sachgütererzeugung \\
\hline 2623 & $\begin{array}{l}\text { Herst. v. Isolatoren u. } \\
\text { Isolierteile a. Keramik }\end{array}$ & $\begin{array}{l}\text { Keramik (o. } \\
\text { Baukeramik) }\end{array}$ & $\begin{array}{l}\text { Herst. u. Bearb. } \\
\text { und Stein }\end{array}$ & v. Glas & Sachgütererzeugung \\
\hline 2624 & $\begin{array}{l}\text { Herst. v. keram. Erzeugnis- } \\
\text { sen für techn. Zwecke }\end{array}$ & $\begin{array}{l}\text { Keramik (o. Ziegelei u. } \\
\text { Baukeramik) }\end{array}$ & $\begin{array}{l}\text { Herst. u. Bearb. } \\
\text { und Stein }\end{array}$ & v. Glas & Sachgütererzeugung \\
\hline 2625 & $\begin{array}{l}\text { Herst. v. keramischen } \\
\text { Erzeugnissen a. n. g. }\end{array}$ & $\begin{array}{l}\text { Keramik (o. Ziegelei u. } \\
\text { Baukeramik) }\end{array}$ & $\begin{array}{l}\text { Herst. u. Bearb. } \\
\text { und Stein }\end{array}$ & v. Glas & Sachgütererzeugung \\
\hline 2626 & $\begin{array}{l}\text { Herst. v. feuerfesten } \\
\text { keramischen Werkstoffen }\end{array}$ & $\begin{array}{l}\text { Keramik (o. Ziegelei u. } \\
\text { Baukeramik) }\end{array}$ & $\begin{array}{l}\text { Herst. u. Bearb. } \\
\text { und Stein }\end{array}$ & v. Glas & Sachgütererzeugung \\
\hline 2630 & $\begin{array}{l}\text { Herst. v. keramischen } \\
\text { Wand- u. Bodenfliesen }\end{array}$ & $\begin{array}{l}\text { Herst. v. keramischen } \\
\text { Wand- u. Bodenfliesen }\end{array}$ & $\begin{array}{l}\text { Herst. u. Bearb. } \\
\text { und Stein }\end{array}$ & v. Glas & Sachgütererzeugung \\
\hline 2640 & $\begin{array}{l}\text { Ziegelei, Herstellung v. } \\
\text { sonst. Baukeramik }\end{array}$ & $\begin{array}{l}\text { Ziegelei, Herst. v. sonst. } \\
\text { Baukeramik }\end{array}$ & $\begin{array}{l}\text { Herst. u. Bearb. } \\
\text { und Stein }\end{array}$ & v. Glas & Sachgütererzeugung \\
\hline 2651 & Herstellung von Zement & $\begin{array}{l}\text { Herst. v. Zement, Kalk u. } \\
\text { gebranntem Gips }\end{array}$ & $\begin{array}{l}\text { Herst. u. Bearb. } \\
\text { und Stein }\end{array}$ & v. Glas & Sachgütererzeugung \\
\hline 2652 & Herstellung von Kalk & $\begin{array}{l}\text { Herst. v. Zement, Kalk u. } \\
\text { gebranntem Gips }\end{array}$ & $\begin{array}{l}\text { Herst. u. Bearb. } \\
\text { und Stein }\end{array}$ & v. Glas & Sachgütererzeugung \\
\hline 2653 & Herst. von gebranntem Gips & $\begin{array}{l}\text { Herst. v. Zement, Kalk u. } \\
\text { gebranntem Gips }\end{array}$ & $\begin{array}{l}\text { Herst. u. Bearb. } \\
\text { und Stein }\end{array}$ & v. Glas & Sachgütererzeugung \\
\hline 2661 & $\begin{array}{l}\text { Herst. v. Betonerzeugnissen } \\
\text { f. d. Bau }\end{array}$ & $\begin{array}{l}\text { Herst. v. Erzeugn. aus Be- } \\
\text { ton, Zement }\end{array}$ & $\begin{array}{l}\text { Herst. u. Bearb. } \\
\text { und Stein }\end{array}$ & v. Glas & Sachgütererzeugung \\
\hline 2662 & $\begin{array}{l}\text { Herst. v. Gipserzeugnissen } \\
\text { für den Bau }\end{array}$ & $\begin{array}{l}\text { Herst. v. Erzeugn. aus Be- } \\
\text { ton, Zement }\end{array}$ & $\begin{array}{l}\text { Herst. u. Bearb. } \\
\text { und Stein }\end{array}$ & v. Glas & Sachgütererzeugung \\
\hline 2663 & Herst. von Transportbeton & $\begin{array}{l}\text { Herst. v. Erzeugn. aus Be- } \\
\text { ton, Zement }\end{array}$ & $\begin{array}{l}\text { Herst. u. Bearb. } \\
\text { und Stein }\end{array}$ & v. Glas & Sachgütererzeugung \\
\hline 2664 & Herst. von Mörtel & $\begin{array}{l}\text { Herst. v. Erzeugn. aus Be- } \\
\text { ton, Zement }\end{array}$ & $\begin{array}{l}\text { Herst. u. Bearb. } \\
\text { und Stein }\end{array}$ & v. Glas & Sachgütererzeugung \\
\hline 2665 & $\begin{array}{l}\text { Herst. von Faserzement- } \\
\text { waren }\end{array}$ & $\begin{array}{l}\text { Herst. v. Erzeugn. aus Be- } \\
\text { ton, Zement }\end{array}$ & $\begin{array}{l}\text { Herst. u. Bearb. } \\
\text { und Stein }\end{array}$ & v. Glas & Sachgütererzeugung \\
\hline 2666 & $\begin{array}{l}\text { Herst. v. Erzeugnissen a. } \\
\text { Beton, Zement u. Gips }\end{array}$ & $\begin{array}{l}\text { Herst. v. Erzeugn. aus Be- } \\
\text { ton, Zement }\end{array}$ & $\begin{array}{l}\text { Herst. u. Bearb. } \\
\text { und Stein }\end{array}$ & v. Glas & Sachgütererzeugung \\
\hline 2670 & $\begin{array}{l}\text { Be- und Verarbeitung von } \\
\text { Natursteinen a. n. g }\end{array}$ & $\begin{array}{l}\text { Be- und Verarbeitung von } \\
\text { Natursteinen a. n. g }\end{array}$ & $\begin{array}{l}\text { Herst. u. Bearb. } \\
\text { und Stein }\end{array}$ & v. Glas & Sachgütererzeugung \\
\hline 2681 & $\begin{array}{l}\text { Herst. v. Mühlsteinen, } \\
\text { Steinen zum Zerfasern }\end{array}$ & $\begin{array}{l}\text { Herst. v. Mineralerzeugnis- } \\
\text { sen }\end{array}$ & $\begin{array}{l}\text { Herst. u. Bearb. } \\
\text { und Stein }\end{array}$ & v. Glas & Sachgütererzeugung \\
\hline 2682 & $\begin{array}{l}\text { Herst. v. Mineralerzeugnis- } \\
\text { sen a. n. g. }\end{array}$ & $\begin{array}{l}\text { Herst. v. Mineralerzeugnis- } \\
\text { sen }\end{array}$ & $\begin{array}{l}\text { Herst. u. Bearb. } \\
\text { und Stein }\end{array}$ & v. Glas & Sachgütererzeugung \\
\hline 2710 & $\begin{array}{l}\text { Erz. von Roheisen, Stahl u. } \\
\text { Ferrolegierung }\end{array}$ & $\begin{array}{l}\text { Erz. von Roheisen, Stahl u. } \\
\text { Ferrolegierung }\end{array}$ & $\begin{array}{l}\text { Metallerzeugung } \mathrm{c} \\
\text { beitung }\end{array}$ & a. - bear- & Sachgütererzeugung \\
\hline 2721 & $\begin{array}{l}\text { Herstellung von Rohren, } \\
\text { Rohrform-, Rohrvers }\end{array}$ & Herstellung von Rohren & $\begin{array}{l}\text { Metallerzeugung } x \\
\text { beitung }\end{array}$ & a. - bear- & Sachgütererzeugung \\
\hline 2722 & $\begin{array}{l}\text { Herstellung von Stahlrohren, } \\
\text { Rohrform-, Roh }\end{array}$ & Herstellung von Rohren & $\begin{array}{l}\text { Metallerzeugung } c \\
\text { beitung }\end{array}$ & a. - bear- & Sachgütererzeugung \\
\hline 2731 & Herstellung von Blankstahl & $\begin{array}{l}\text { Sonst. erste Bearb. von } \\
\text { Eisen und Stahl }\end{array}$ & $\begin{array}{l}\text { Metallerzeugung } c \\
\text { beitung }\end{array}$ & a. - bear- & Sachgütererzeugung \\
\hline 2732 & $\begin{array}{l}\text { Herstellung von Kaltband } \\
\text { unter } 500 \mathrm{~mm} \text { Breit }\end{array}$ & $\begin{array}{l}\text { Sonst. erste Bearb. von } \\
\text { Eisen und Stahl }\end{array}$ & $\begin{array}{l}\text { Metallerzeugung } x \\
\text { beitung }\end{array}$ & a. - bear- & Sachgütererzeugung \\
\hline 2733 & Herstellung von Kaltprofilen & $\begin{array}{l}\text { Sonst. erste Bearb. von } \\
\text { Eisen und Stahl }\end{array}$ & $\begin{array}{l}\text { Metallerzeugung } x \\
\text { beitung }\end{array}$ & d. - bear- & Sachgütererzeugung \\
\hline 2734 & $\begin{array}{l}\text { Herstellung von gezogenem } \\
\text { Draht }\end{array}$ & $\begin{array}{l}\text { Sonst. erste Bearb. von } \\
\text { Eisen und Stahl }\end{array}$ & $\begin{array}{l}\text { Metallerzeugung } \mathrm{l} \\
\text { beitung }\end{array}$ & a. - bear- & Sachgütererzeugung \\
\hline 2735 & $\begin{array}{l}\text { Erz. von Roheisen, Stahl u. } \\
\text { Ferrolegierung }\end{array}$ & $\begin{array}{l}\text { Erz. von Roheisen, Stahl u. } \\
\text { Ferrolegierung }\end{array}$ & $\begin{array}{l}\text { Metallerzeugung } x \\
\text { beitung }\end{array}$ & a. - bear- & Sachgütererzeugung \\
\hline 2741 & $\begin{array}{l}\text { Erz. u. erste Bearbeitung } \\
\text { von Edelmetall }\end{array}$ & $\begin{array}{l}\text { Erz. u. erste Bearb. von } \\
\text { NE- Metallen }\end{array}$ & $\begin{array}{l}\text { Metallerzeugung } c \\
\text { beitung }\end{array}$ & a. - bear- & Sachgütererzeugung \\
\hline
\end{tabular}

Continued on next page... 


\begin{tabular}{|c|c|c|c|c|}
\hline$\overline{\text { code }}$ & $\begin{array}{l}\text { industry } \\
\text { (very narrow) }\end{array}$ & $\begin{array}{l}\text { industry } \\
\text { (narrow) }\end{array}$ & $\begin{array}{l}\text { industry } \\
\text { (broad) }\end{array}$ & $\begin{array}{l}\text { industry } \\
\text { (very broad) }\end{array}$ \\
\hline 2742 & $\begin{array}{l}\text { Erz. u. erste Bearbeitung } \\
\text { von Aluminium }\end{array}$ & $\begin{array}{l}\text { Erz. u. erste Bearb. von } \\
\text { NE- Metallen }\end{array}$ & $\begin{array}{l}\text { Metallerzeugung u. - bear- } \\
\text { beitung }\end{array}$ & Sachgütererzeugung \\
\hline 2743 & $\begin{array}{l}\text { Erz. u. erste Bearbeitung } \\
\text { von Blei, Zink }\end{array}$ & $\begin{array}{l}\text { Erz. u. erste Bearb. von } \\
\text { NE- Metallen }\end{array}$ & $\begin{array}{l}\text { Metallerzeugung u. - bear- } \\
\text { beitung }\end{array}$ & Sachgütererzeugung \\
\hline 2744 & $\begin{array}{l}\text { Erz. u. erste Bearbeitung } \\
\text { von Kupfer }\end{array}$ & $\begin{array}{l}\text { Erz. u. erste Bearb. von } \\
\text { NE- Metallen }\end{array}$ & $\begin{array}{l}\text { Metallerzeugung u. - bear- } \\
\text { beitung }\end{array}$ & Sachgütererzeugung \\
\hline 2745 & $\begin{array}{l}\text { Erz. u. erste Bearb. von } \\
\text { sonst. NE- Metallen }\end{array}$ & $\begin{array}{l}\text { Erz. u. erste Bearb. von } \\
\text { NE- Metallen }\end{array}$ & $\begin{array}{l}\text { Metallerzeugung u. - bear- } \\
\text { beitung }\end{array}$ & Sachgütererzeugung \\
\hline 2751 & Eisengießerei & Gießereiindustrie & $\begin{array}{l}\text { Metallerzeugung u. - bear- } \\
\text { beitung }\end{array}$ & Sachgütererzeugung \\
\hline 2752 & Stahlgießerei & Gießereiindustrie & $\begin{array}{l}\text { Metallerzeugung u. - bear- } \\
\text { beitung }\end{array}$ & Sachgütererzeugung \\
\hline 2753 & Leichtmetallgießerei & Gießereiindustrie & $\begin{array}{l}\text { Metallerzeugung u. - bear- } \\
\text { beitung }\end{array}$ & Sachgütererzeugung \\
\hline 2754 & Schwermetallgießerei & Gießereiindustrie & $\begin{array}{l}\text { Metallerzeugung u. - bear- } \\
\text { beitung }\end{array}$ & Sachgütererzeugung \\
\hline 2811 & $\begin{array}{l}\text { Herst. v. Stahl- u. Leicht- } \\
\text { metallkonstruktionen }\end{array}$ & Stahl- u. Leichtmetallbau & Herst. v. Metallerzeugnissen & Sachgütererzeugung \\
\hline 2812 & $\begin{array}{l}\text { Herst. v. Ausbauelementen } \\
\text { aus Stahl u. Leichtm. }\end{array}$ & Stahl- u. Leichtmetallbau & Herst. v. Metallerzeugnissen & Sachgütererzeugung \\
\hline 2821 & $\begin{array}{l}\text { Herst. von Tanks und Sam- } \\
\text { melbehältern }\end{array}$ & Kessel u. Behälterbau & Herst. v. Metallerzeugnissen & Sachgütererzeugung \\
\hline 2822 & $\begin{array}{l}\text { Herst. von Heizkörpern für } \\
\text { Zentralheiz }\end{array}$ & Kessel u. Behälterbau & Herst. v. Metallerzeugnissen & Sachgütererzeugung \\
\hline 2830 & $\begin{array}{l}\text { Herst. von Dampfkesseln } \\
\text { (ohne Zentralh }\end{array}$ & Herst. von Dampfkesseln & Herst. v. Metallerzeugnissen & Sachgütererzeugung \\
\hline 2840 & $\begin{array}{l}\text { Herst. von Schmiede-, Preß- } \\
\text { u. Stanzteilen }\end{array}$ & $\begin{array}{l}\text { Herst. von Schmiede-, Preß- } \\
\text { u. Stanzteilen }\end{array}$ & Herst. v. Metallerzeugnissen & Sachgütererzeugung \\
\hline 2851 & $\begin{array}{l}\text { Oberflächenveredlung und } \\
\text { Wärmebehandlung }\end{array}$ & $\begin{array}{l}\text { Oberflächenveredlung, } \\
\text { Wärmebehandlung } \\
\text { Mechanik }\end{array}$ & Herst. v. Metallerzeugnissen & Sachgütererzeugung \\
\hline 2852 & Mechanik a. n. g. & $\begin{array}{l}\text { Oberflächenveredlung, } \\
\text { Wärmebehandlung } \\
\text { Mechanik }\end{array}$ & Herst. v. Metallerzeugnissen & Sachgütererzeugung \\
\hline 2861 & $\begin{array}{l}\text { Herst. von Schneidwaren } \\
\text { und Bestecken }\end{array}$ & $\begin{array}{l}\text { Herst. v. Schneidewaren, } \\
\text { Werkzeuge u. Schlösser }\end{array}$ & Herst. v. Metallerzeugnissen & Sachgütererzeugung \\
\hline 2862 & Hers. von Werkzeugen & $\begin{array}{l}\text { Herst. v. Schneidewaren, } \\
\text { Werkzeuge u. Schlösser }\end{array}$ & Herst. v. Metallerzeugnissen & Sachgütererzeugung \\
\hline 2863 & $\begin{array}{l}\text { Herst. von Schlössern und } \\
\text { Beschlägen }\end{array}$ & $\begin{array}{l}\text { Herst. v. Schneidewaren, } \\
\text { Werkzeuge u. Schlösser }\end{array}$ & Herst. v. Metallerzeugnissen & Sachgütererzeugung \\
\hline 2871 & $\begin{array}{l}\text { Herst. v. Behältern aus } \\
\text { Eisen und Stahl }\end{array}$ & $\begin{array}{l}\text { Herst. v. sonst. Eisen- } \\
\text { Blech- u. Metallwaren }\end{array}$ & Herst. v. Metallerzeugnissen & Sachgütererzeugung \\
\hline 2872 & $\begin{array}{l}\text { Herst. v. Verpackungen und } \\
\text { Verschlüssen }\end{array}$ & $\begin{array}{l}\text { Herst. v. sonst. Eisen- } \\
\text { Blech- u. Metallwaren }\end{array}$ & Herst. v. Metallerzeugnissen & Sachgütererzeugung \\
\hline 2873 & Herst. von Drahtwaren & $\begin{array}{l}\text { Herst. v. sonst. Eisen- } \\
\text { Blech- u. Metallwaren }\end{array}$ & Herst. v. Metallerzeugnissen & Sachgütererzeugung \\
\hline 2874 & $\begin{array}{l}\text { Herst. v. Schrauben, Nieten, } \\
\text { Ketten u. Federn }\end{array}$ & $\begin{array}{l}\text { Herst. v. sonst. Eisen- } \\
\text { Blech- u. Metallwaren }\end{array}$ & Herst. v. Metallerzeugnissen & Sachgütererzeugung \\
\hline 2875 & $\begin{array}{l}\text { Herst. v. Eisen-, Blech- u. } \\
\text { Metallwaren }\end{array}$ & $\begin{array}{l}\text { Herst. v. sonst. Eisen- } \\
\text { Blech- u. Metallwaren }\end{array}$ & Herst. v. Metallerzeugnissen & Sachgütererzeugung \\
\hline 2911 & $\begin{array}{l}\text { Herst. v. Verbrennungsmo- } \\
\text { toren u. Turbinen }\end{array}$ & $\begin{array}{l}\text { Herst. v. Masch. f. d. Erz. } \\
\text { u. Nutzung v. mech. En- } \\
\text { ergie }\end{array}$ & Maschinenbau & Sachgütererzeugung \\
\hline 2912 & $\begin{array}{l}\text { Herst. v. Pumpen und Kom- } \\
\text { pressoren }\end{array}$ & $\begin{array}{l}\text { Herst. v. Masch. f. d. Erz. } \\
\text { u. Nutzung v. mech. En- } \\
\text { ergie }\end{array}$ & Maschinenbau & Sachgütererzeugung \\
\hline 2913 & Herst. von Armaturen & $\begin{array}{l}\text { Herst. v. Masch. f. d. Erz. } \\
\text { u. Nutzung v. mech. En- } \\
\text { ergie }\end{array}$ & Maschinenbau & Sachgütererzeugung \\
\hline 2914 & $\begin{array}{l}\text { Herst. v. Lagern, Getrieben } \\
\text { u. Zahnrädern }\end{array}$ & $\begin{array}{l}\text { Herst. v. Masch. f. d. Erz. } \\
\text { u. Nutzung v. mech. En- } \\
\text { ergie }\end{array}$ & Maschinenbau & Sachgütererzeugung \\
\hline
\end{tabular}




\begin{tabular}{|c|c|c|c|c|}
\hline code & $\begin{array}{l}\text { industry } \\
\text { (very narrow) }\end{array}$ & $\begin{array}{l}\text { industry } \\
\text { (narrow) }\end{array}$ & $\begin{array}{l}\text { industry } \\
\text { (broad) }\end{array}$ & $\begin{array}{l}\text { industry } \\
\text { (very broad) }\end{array}$ \\
\hline 2921 & $\begin{array}{l}\text { Herst. von Öfen und Bren- } \\
\text { nern }\end{array}$ & $\begin{array}{l}\text { Herst. v. sonst. Masch. un- } \\
\text { spezif. Verwendung }\end{array}$ & Maschinenbau & Sachgütererzeugung \\
\hline 2922 & $\begin{array}{l}\text { Herst. von Hebezeugen und } \\
\text { Fördermittel }\end{array}$ & $\begin{array}{l}\text { Herst. v. sonst. Masch. un- } \\
\text { spezif. Verwendung }\end{array}$ & Maschinenbau & Sachgütererzeugung \\
\hline 2923 & $\begin{array}{l}\text { Herst. v. kälte- u. lufttech- } \\
\text { nischen Erzeugn. }\end{array}$ & $\begin{array}{l}\text { Herst. v. sonst. Masch. un- } \\
\text { spezif. Verwendung }\end{array}$ & Maschinenbau & Sachgütererzeugung \\
\hline 2924 & $\begin{array}{l}\text { Herst. v. Masch. unspezifis- } \\
\text { cher Verwendung }\end{array}$ & $\begin{array}{l}\text { Herst. v. sonst. Masch. un- } \\
\text { spezif. Verwendung }\end{array}$ & Maschinenbau & Sachgütererzeugung \\
\hline 2931 & Herst. von Ackerschleppern & $\begin{array}{l}\text { Herst. v. land- u. forstw. } \\
\text { Maschinen }\end{array}$ & Maschinenbau & Sachgütererzeugung \\
\hline 2932 & $\begin{array}{l}\text { Herst. v. sonst. land- u. } \\
\text { forstwirt. Maschinen }\end{array}$ & $\begin{array}{l}\text { Herst. v. land- u. forstw. } \\
\text { Maschinen }\end{array}$ & Maschinenbau & Sachgütererzeugung \\
\hline 2940 & $\begin{array}{l}\text { Herst. von Werkzeugmaschi- } \\
\text { nen(bis } 7 / 04 \text { ) }\end{array}$ & $\begin{array}{l}\text { Herst. von Werkzeugmaschi- } \\
\text { nen }\end{array}$ & Maschinenbau & Sachgütererzeugung \\
\hline 2941 & $\begin{array}{l}\text { Herst. v. handgeführten } \\
\text { Werkzeugen m. Mo- } \\
\text { torantrieb }\end{array}$ & $\begin{array}{l}\text { Herst. von Werkzeugmaschi- } \\
\text { nen }\end{array}$ & Maschinenbau & Sachgütererzeugung \\
\hline 2942 & $\begin{array}{l}\text { Herst. v. sonst. Werkzeug- } \\
\text { masch. f. d. Metallbear- } \\
\text { beitung }\end{array}$ & $\begin{array}{l}\text { Herst. von Werkzeugmaschi- } \\
\text { nen }\end{array}$ & Maschinenbau & Sachgütererzeugung \\
\hline 2943 & $\begin{array}{l}\text { Herst. v. Werkzeugmaschi- } \\
\text { nen a. n. g. }\end{array}$ & $\begin{array}{l}\text { Herst. von Werkzeugmaschi- } \\
\text { nen }\end{array}$ & Maschinenbau & Sachgütererzeugung \\
\hline 2951 & $\begin{array}{l}\text { Herst. v. Maschinen für die } \\
\text { Metallerzeugung }\end{array}$ & $\begin{array}{l}\text { Herst. v. Masch. für sonst. } \\
\text { Wirtschaftszweige }\end{array}$ & Maschinenbau & Sachgütererzeugung \\
\hline 2952 & $\begin{array}{l}\text { Herst. v. Bergwerks-, Bau- } \\
\text { u. Baustoffmasch. }\end{array}$ & $\begin{array}{l}\text { Herst. v. Masch. für sonst. } \\
\text { Wirtschaftszweige }\end{array}$ & Maschinenbau & Sachgütererzeugung \\
\hline 2953 & $\begin{array}{l}\text { Herst. v. Masch. für die } \\
\text { Nahrungs- u. Genußm. erz. }\end{array}$ & $\begin{array}{l}\text { Herst. v. Masch. für sonst. } \\
\text { Wirtschaftszweige }\end{array}$ & Maschinenbau & Sachgütererzeugung \\
\hline 2954 & $\begin{array}{l}\text { Herst. v. Maschinen für die } \\
\text { Textil- u }\end{array}$ & $\begin{array}{l}\text { Herst. v. Masch. für sonst. } \\
\text { Wirtschaftszweige }\end{array}$ & Maschinenbau & Sachgütererzeugung \\
\hline 2955 & Herst. von Papiermaschinen & $\begin{array}{l}\text { Herst. v. Masch. für sonst. } \\
\text { Wirtschaftszweige }\end{array}$ & Maschinenbau & Sachgütererzeugung \\
\hline 2956 & $\begin{array}{l}\text { Herst. v. Masch. für sonst. } \\
\text { Wirtschaftszweige }\end{array}$ & $\begin{array}{l}\text { Herst. v. Masch. für sonst. } \\
\text { Wirtschaftszweige }\end{array}$ & Maschinenbau & Sachgütererzeugung \\
\hline 2960 & $\begin{array}{l}\text { Herst. von Waffen und } \mathrm{Mu}- \\
\text { nition }\end{array}$ & $\begin{array}{l}\text { Herst. von Waffen u. Muni- } \\
\text { tion }\end{array}$ & Maschinenbau & Sachgütererzeugung \\
\hline 2971 & $\begin{array}{l}\text { Herst. v. elektri. u. elek- } \\
\text { trotherm. Haushaltsgeräten }\end{array}$ & Herst. von Haushaltsgeräten & Maschinenbau & Sachgütererzeugung \\
\hline 2972 & $\begin{array}{l}\text { Herst. v. nichtelektri. } \\
\text { Koch- u. Heizgeräten }\end{array}$ & Herst. von Haushaltsgeräten & Maschinenbau & Sachgütererzeugung \\
\hline 3001 & Herst. von Büromaschinen & $\begin{array}{l}\text { Herst. v. Bürom. - u. } \\
\text { Datenverarbeitungsgeräten }\end{array}$ & $\begin{array}{l}\text { Herst. v. Büromasch. , } \\
\text { Datenverarbeitungsgeräten }\end{array}$ & Sachgütererzeugung \\
\hline 3002 & $\begin{array}{l}\text { Herst. v. Datenverar- } \\
\text { beitungsgeräten }\end{array}$ & $\begin{array}{l}\text { Herst. v. Bürom. - u. } \\
\text { Datenverarbeitungsgeräten }\end{array}$ & $\begin{array}{l}\text { Herst. v. Büromasch. , } \\
\text { Datenverarbeitungsgeräten }\end{array}$ & Sachgütererzeugung \\
\hline 3110 & $\begin{array}{l}\text { Herst. v. Elektromotoren, } \\
\text { Generatoren u. Transf. }\end{array}$ & $\begin{array}{l}\text { Herst. v. Elektromotoren, } \\
\text { Generatoren u. Transf. }\end{array}$ & $\begin{array}{l}\text { Herst. v. Geräten der Elek- } \\
\text { trizitätserzeugung }\end{array}$ & Sachgütererzeugung \\
\hline 3120 & $\begin{array}{l}\text { Herst. } \quad \mathrm{v} . \quad \text { Elek- } \\
\text { trizitätsverteilungseinrichtungen }\end{array}$ & $\begin{array}{l}\text { Herst. v. } \quad \text { Elek- } \\
\text { trizitätsverteilungseinrichtungen }\end{array}$ & $\begin{array}{l}\text { Herst. v. Geräten der Elek- } \\
\text { trizitätserzeugung }\end{array}$ & Sachgütererzeugung \\
\hline 3130 & $\begin{array}{l}\text { Herst. v. isolierten Elek- } \\
\text { trokabeln }\end{array}$ & $\begin{array}{l}\text { Herst. v. isolierten Elek- } \\
\text { trokabeln }\end{array}$ & $\begin{array}{l}\text { Herst. v. Geräten der Elek- } \\
\text { trizitätserzeugung }\end{array}$ & Sachgütererzeugung \\
\hline 3140 & $\begin{array}{l}\text { Herst. v. Akkumulatoren } \\
\text { und Batterien }\end{array}$ & $\begin{array}{l}\text { Herst. v. Akkumulatoren } \\
\text { und Batterien }\end{array}$ & $\begin{array}{l}\text { Herst. v. Geräten der Elek- } \\
\text { trizitätserzeugung }\end{array}$ & Sachgütererzeugung \\
\hline 3150 & $\begin{array}{l}\text { Herst. v. elektrischen Lam- } \\
\text { pen u. Leuchten }\end{array}$ & $\begin{array}{l}\text { Herst. v. elektrischen Lam- } \\
\text { pen u. Leuchten }\end{array}$ & $\begin{array}{l}\text { Herst. v. Geräten der Elek- } \\
\text { trizitätserzeugung }\end{array}$ & Sachgütererzeugung \\
\hline 3161 & $\begin{array}{l}\text { Herst. v. } \quad \text { elektr. } \\
\text { Ausrüstungen f. Motoren }\end{array}$ & $\begin{array}{l}\text { Herst. v. elektrischen } \\
\text { Ausrüstungen }\end{array}$ & $\begin{array}{l}\text { Herst. v. Geräten der Elek- } \\
\text { trizitätserzeugung }\end{array}$ & Sachgütererzeugung \\
\hline 3162 & $\begin{array}{l}\text { Herst. v. sonst. elektri. } \\
\text { Ausrüstungen }\end{array}$ & $\begin{array}{l}\text { Herst. v. elektrischen } \\
\text { Ausrüstungen }\end{array}$ & $\begin{array}{l}\text { Herst. v. Geräten der Elek- } \\
\text { trizitätserzeugung }\end{array}$ & Sachgütererzeugung \\
\hline 3210 & $\begin{array}{l}\text { Herst. v. elektron. Bauele- } \\
\text { menten }\end{array}$ & $\begin{array}{l}\text { Herst. v. elektron. Bauele- } \\
\text { menten }\end{array}$ & $\begin{array}{l}\text { Rundfunk- , Fernseh- u. } \\
\text { Nachrichtentechnik }\end{array}$ & Sachgütererzeugung \\
\hline 3220 & $\begin{array}{l}\text { Herst. v. nachrichtentechn. } \\
\text { Geräten }\end{array}$ & $\begin{array}{l}\text { Herst. v. nachrichtentechn. } \\
\text { Geräten }\end{array}$ & $\begin{array}{l}\text { Rundfunk- , Fernseh- u. } \\
\text { Nachrichtentechnik }\end{array}$ & Sachgütererzeugung \\
\hline
\end{tabular}

Continued on next page... 


\begin{tabular}{|c|c|c|c|c|}
\hline code & $\begin{array}{l}\text { industry } \\
\text { (very narrow) }\end{array}$ & $\begin{array}{l}\text { industry } \\
\text { (narrow) }\end{array}$ & $\begin{array}{l}\text { industry } \\
\text { (broad) }\end{array}$ & $\begin{array}{l}\text { industry } \\
\text { (very broad) }\end{array}$ \\
\hline 3230 & $\begin{array}{l}\text { Herst. v. Rundfunk- u. } \\
\text { Fernsehgeräten }\end{array}$ & $\begin{array}{l}\text { Herst. v. Rundfunk- u. } \\
\text { Fernsehgeräten }\end{array}$ & $\begin{array}{l}\text { Rundfunk- , Fernseh- u. } \\
\text { Nachrichtentechnik }\end{array}$ & Sachgütererzeugung \\
\hline 3310 & $\begin{array}{l}\text { Herst. v. medizin. Geräten } \\
\text { u. orthop. Vorrichtungen }\end{array}$ & $\begin{array}{l}\text { Herst. v. medizin. Geräten } \\
\text { u. orthop. Vorrichtungen }\end{array}$ & $\begin{array}{ll}\text { Medizin- } \quad, \quad \text { Mess- } & \text { u. } \\
\text { Regelungstechnik, Optik } & \end{array}$ & Sachgütererzeugung \\
\hline 3320 & $\begin{array}{l}\text { Herst. v. Meß-, Kontroll- } \\
\text { Navigationsinstrumenten }\end{array}$ & $\begin{array}{l}\text { Herst. v. Meß-, Kontroll-, } \\
\text { Navigationsinstrumenten }\end{array}$ & $\begin{array}{ll}\text { Medizin- } \quad \text { Mess- } & \text { u. } \\
\text { Regelungstechnik, Optik }\end{array}$ & Sachgütererzeugung \\
\hline 3330 & $\begin{array}{l}\text { Herst. v. industriellen } \\
\text { Prozeßsteuerungsanl. }\end{array}$ & $\begin{array}{l}\text { Herst. v. industriellen } \\
\text { Prozeßsteuerungsanl. }\end{array}$ & $\begin{array}{ll}\text { Medizin- } \quad, \quad \text { Mess- } & \text { u. } \\
\text { Regelungstechnik, Optik } & \end{array}$ & Sachgütererzeugung \\
\hline 3340 & $\begin{array}{l}\text { Herst. v. opti. u. fotografis- } \\
\text { chen Geräten }\end{array}$ & $\begin{array}{l}\text { Herst. v. opti. u. fotografis- } \\
\text { chen Geräten }\end{array}$ & $\begin{array}{l}\text { Medizin- , Mess- } \text { u. } \\
\text { Regelungstechnik, Optik }\end{array}$ & Sachgütererzeugung \\
\hline 3350 & Herst. von Uhren & Herst. von Uhren & $\begin{array}{ll}\text { Medizin- } \quad, \quad \text { Mess- } & \text { u. } \\
\text { Regelungstechnik, Optik } & \end{array}$ & Sachgütererzeugung \\
\hline 3410 & $\begin{array}{l}\text { Herst. v. Kraftwagen u. } \\
\text { Kraftwagenmotoren }\end{array}$ & $\begin{array}{l}\text { Herst. v. Kraftwagen u. } \\
\text { Kraftwagenmotoren }\end{array}$ & $\begin{array}{l}\text { Herst. v. Kraftwagen u. } \\
\text { Kfz- Teilen }\end{array}$ & Sachgütererzeugung \\
\hline 3420 & $\begin{array}{l}\text { Herst. v. Karosserien, Auf- } \\
\text { bauten u. Anhängern }\end{array}$ & $\begin{array}{l}\text { Herst. v. Karosserien, Auf- } \\
\text { bauten u. Anhängern }\end{array}$ & $\begin{array}{l}\text { Herst. v. Kraftwagen u. } \\
\text { Kfz- Teilen }\end{array}$ & Sachgütererzeugung \\
\hline 3430 & $\begin{array}{l}\text { Herst. v. Teilen u. Zubehör } \\
\text { f. Kraftwagen }\end{array}$ & $\begin{array}{l}\text { Herst. v. Teilen u. Zubehör } \\
\text { f. Kraftwagen }\end{array}$ & $\begin{array}{l}\text { Herst. v. Kraftwagen u. } \\
\text { Kfz- Teilen }\end{array}$ & Sachgütererzeugung \\
\hline 3511 & $\begin{array}{l}\text { Schiffbau (o. Boots- u. } \\
\text { Yachtbau) }\end{array}$ & Schiffbau & Sonstiger Fahrzeugbau & Sachgütererzeugung \\
\hline 3512 & Boots- und Yachtbau & Schiffbau & Sonstiger Fahrzeugbau & Sachgütererzeugung \\
\hline 3520 & Schienenfahrzeugbau & Schienenfahrzeugbau & Sonstiger Fahrzeugbau & Sachgütererzeugung \\
\hline 3530 & Luft- und Raumfahrzeugbau & Luft- und Raumfahrzeugbau & Sonstiger Fahrzeugbau & Sachgütererzeugung \\
\hline 3541 & Herstellung von Krafträdern & $\begin{array}{l}\text { Herst. v. Krafträdern, } \\
\text { Fahrrädern u. Behinderten- } \\
\text { fahrz. }\end{array}$ & Sonstiger Fahrzeugbau & Sachgütererzeugung \\
\hline 3542 & Herstellung von Fahrrädern & $\begin{array}{l}\text { Herst. v. Krafträdern, } \\
\text { Fahrrädern u. Behinderten- } \\
\text { fahrz. }\end{array}$ & Sonstiger Fahrzeugbau & Sachgütererzeugung \\
\hline 3543 & $\begin{array}{l}\text { Herst. von Behinderten- } \\
\text { fahrzeugen }\end{array}$ & $\begin{array}{l}\text { Herst. v. Krafträdern, } \\
\text { Fahrrädern u. Behinderten- } \\
\text { fahrz. }\end{array}$ & Sonstiger Fahrzeugbau & Sachgütererzeugung \\
\hline 3550 & Fahrzeugbau a. n. g. & Fahrzeugbau a. n. g. & Sonstiger Fahrzeugbau & Sachgütererzeugung \\
\hline 3611 & Herstellung von Sitzmöbeln & Herstellung von Möbeln & $\begin{array}{l}\text { Möbel, Musikinstrum. } \\
\text { Sport- u. Spielw. }\end{array}$ & Sachgütererzeugung \\
\hline 3612 & $\begin{array}{l}\text { Herstellung von Büro- und } \\
\text { Ladenmöbeln }\end{array}$ & Herstellung von Möbeln & $\begin{array}{l}\text { Möbel, Musikinstrum. } \\
\text { Sport- u. Spielw. }\end{array}$ & Sachgütererzeugung \\
\hline 3613 & $\begin{array}{l}\text { Herstellung von } \\
\text { Küchenmöbeln aus Holz }\end{array}$ & Herstellung von Möbeln & $\begin{array}{l}\text { Möbel, Musikinstrum. } \\
\text { Sport- u. Spielw. }\end{array}$ & Sachgütererzeugung \\
\hline 3614 & $\begin{array}{l}\text { Herstellung von sonstigen } \\
\text { Möbeln }\end{array}$ & Herstellung von Möbeln & $\begin{array}{l}\text { Möbel, Musikinstrum. } \\
\text { Sport- u. Spielw. }\end{array}$ & Sachgütererzeugung \\
\hline 3615 & Herstellung von Matratzen & Herstellung von Möbeln & $\begin{array}{l}\text { Möbel, Musikinstrum. } \\
\text { Sport- u. Spielw. }\end{array}$ & Sachgütererzeugung \\
\hline 3621 & $\begin{array}{l}\text { Herstellung von Münzen und } \\
\text { Medaillen }\end{array}$ & $\begin{array}{l}\text { Herstellung von Schmuck u. } \\
\text { ä. }\end{array}$ & $\begin{array}{l}\text { Möbel, Musikinstrum. } \\
\text { Sport- u. Spielw. }\end{array}$ & Sachgütererzeugung \\
\hline 3622 & $\begin{array}{l}\text { Herstellung von Schmuck, } \\
\text { Gold- und Silbersc }\end{array}$ & $\begin{array}{l}\text { Herstellung von Schmuck u. } \\
\text { ä. }\end{array}$ & $\begin{array}{l}\text { Möbel, Musikinstrum. } \\
\text { Sport- u. Spielw. }\end{array}$ & Sachgütererzeugung \\
\hline 3630 & $\begin{array}{l}\text { Herstellung von Musikin- } \\
\text { strumenten }\end{array}$ & $\begin{array}{l}\text { Herstellung von Musikin- } \\
\text { strumenten }\end{array}$ & $\begin{array}{l}\text { Möbel, Musikinstrum. } \\
\text { Sport- u. Spielw. }\end{array}$ & Sachgütererzeugung \\
\hline 3640 & $\begin{array}{l}\text { Herstellung von Sport- } \\
\text { geräten }\end{array}$ & $\begin{array}{l}\text { Herstellung von Sport- } \\
\text { geräten }\end{array}$ & $\begin{array}{l}\text { Möbel, Musikinstrum. } \\
\text { Sport- u. Spielw. }\end{array}$ & Sachgütererzeugung \\
\hline 3650 & Herstellung von Spielwaren & Herstellung von Spielwaren & $\begin{array}{l}\text { Möbel, Musikinstrum. } \\
\text { Sport- u. Spielw. }\end{array}$ & Sachgütererzeugung \\
\hline 3661 & $\begin{array}{l}\text { Herstellung von Phantasi- } \\
\text { eschmuck }\end{array}$ & $\begin{array}{l}\text { Herstellung von Erzeugnis- } \\
\text { sen }\end{array}$ & $\begin{array}{l}\text { Möbel, Musikinstrum. } \\
\text { Sport- u. Spielw. }\end{array}$ & Sachgütererzeugung \\
\hline 3662 & $\begin{array}{l}\text { Herstellung von Besen und } \\
\text { Bürsten }\end{array}$ & $\begin{array}{l}\text { Herstellung von Erzeugnis- } \\
\text { sen }\end{array}$ & $\begin{array}{l}\text { Möbel, Musikinstrum. } \\
\text { Sport- u. Spielw. }\end{array}$ & Sachgütererzeugung \\
\hline 3663 & $\begin{array}{l}\text { Herstellung von Erzeugnis- } \\
\text { sen a. n. g. }\end{array}$ & $\begin{array}{l}\text { Herstellung von Erzeugnis- } \\
\text { sen }\end{array}$ & $\begin{array}{l}\text { Möbel, Musikinstrum. } \\
\text { Sport- u. Spielw. }\end{array}$ & Sachgütererzeugung \\
\hline 3710 & Rückgewinnung von Schrott & Rückgewinnung von Schrott & Rückgewinnung (Recycling) & Sachgütererzeugung \\
\hline 3720 & $\begin{array}{l}\text { Rückgew. v. nichtmetalli. } \\
\text { Altmaterial u. Rohstoffen }\end{array}$ & $\begin{array}{l}\text { Rückgew. v. nichtmetalli. } \\
\text { Altmaterial u. Rohstoffen }\end{array}$ & Rückgewinnung (Recycling) & Sachgütererzeugung \\
\hline
\end{tabular}

Continued on next page... 


\begin{tabular}{|c|c|c|c|c|c|c|}
\hline \multirow{2}{*}{$\begin{array}{l}\text { code } \\
4010\end{array}$} & \multirow{2}{*}{$\begin{array}{l}\text { industry } \\
\text { (very narrow) } \\
\text { Elektrizitätsversorgung (bis } \\
7 / 04)\end{array}$} & \multirow{2}{*}{$\begin{array}{l}\begin{array}{l}\text { industry } \\
\text { (narrow) }\end{array} \\
\text { Elektrizitätsversorgung }\end{array}$} & \multicolumn{2}{|l|}{$\begin{array}{l}\text { industry } \\
\text { (broad) }\end{array}$} & \multicolumn{2}{|l|}{$\begin{array}{l}\text { industry } \\
\text { (very broad) }\end{array}$} \\
\hline & & & Energieversorgung & & $\begin{array}{l}\text { Energie- und } \\
\text { sorgung }\end{array}$ & Wasserver- \\
\hline 4011 & Elektrizitätsversorgung & Elektrizitätsversorgung & Energieversorgung & & $\begin{array}{l}\text { Energie- und } \\
\text { sorgung }\end{array}$ & Wasserver- \\
\hline 4012 & Elektrizitätsübertragung & Elektrizitätsversorgung & Energieversorgung & & $\begin{array}{l}\text { Energie- und } \\
\text { sorgung }\end{array}$ & Wasserver- \\
\hline 4013 & $\begin{array}{l}\text { Elektrizitätsverteilung und - } \\
\text { handel }\end{array}$ & Elektrizitätsversorgung & Energieversorgung & & $\begin{array}{l}\text { Energie- und } \\
\text { sorgung }\end{array}$ & Wasserver- \\
\hline 4020 & Gasversorgung (bis $7 / 04$ ) & Gasversorgung & Energieversorgung & & $\begin{array}{l}\text { Energie- und } \\
\text { sorgung }\end{array}$ & Wasserver- \\
\hline 4021 & Gaserzeugung & Gasversorgung & Energieversorgung & & $\begin{array}{l}\text { Energie- und } \\
\text { sorgung }\end{array}$ & Wasserver- \\
\hline 4022 & $\begin{array}{l}\text { Gasverteilung u. - handel d. } \\
\text { Rohrleitungen }\end{array}$ & Gasversorgung & Energieversorgung & & $\begin{array}{l}\text { Energie- und } \\
\text { sorgung }\end{array}$ & Wasserver- \\
\hline 4030 & Fernwärmeversorgung & Fernwärmeversorgung & Energieversorgung & & $\begin{array}{l}\text { Energie- und } \\
\text { sorgung }\end{array}$ & Wasserver- \\
\hline 4100 & Wasserversorgung & Wasserversorgung & Wasserversorgung & & $\begin{array}{l}\text { Energie- und } \\
\text { sorgung }\end{array}$ & Wasserver- \\
\hline 4511 & $\begin{array}{l}\text { Abbruch-, Spreng- u. Erd- } \\
\text { bewegungsarbeiten }\end{array}$ & $\begin{array}{l}\text { Vorbereitende Baustellenar- } \\
\text { beiten }\end{array}$ & Bauwesen & & Bauwesen & \\
\hline 4512 & Test- und Suchbohrung & $\begin{array}{l}\text { Vorbereitende Baustellenar- } \\
\text { beiten }\end{array}$ & Bauwesen & & Bauwesen & \\
\hline 4520 & $\begin{array}{l}\text { Hoch- u. Tiefbau Sparte un- } \\
\text { bekannt }\end{array}$ & $\begin{array}{l}\text { Hoch- u. Tiefbau Sparte un- } \\
\text { bekannt }\end{array}$ & Bauwesen & & Bauwesen & \\
\hline 4521 & $\begin{array}{l}\text { Hochbau, Brücken- und Tun- } \\
\text { nelbau u. ä. }\end{array}$ & Hoch- und Tiefbau & Bauwesen & & Bauwesen & \\
\hline 4522 & $\begin{array}{l}\text { Zimmerei, Dachdeckerei, } \\
\text { Bauspenglerei }\end{array}$ & Hoch- und Tiefbau & Bauwesen & & Bauwesen & \\
\hline 4523 & $\begin{array}{l}\text { Straßenbau und Eisenbah- } \\
\text { noberbau }\end{array}$ & Hoch- und Tiefbau & Bauwesen & & Bauwesen & \\
\hline 4524 & Wasserbau & Hoch- und Tiefbau & Bauwesen & & Bauwesen & \\
\hline 4525 & $\begin{array}{l}\text { Spezialbau und sonstiger } \\
\text { Tiefbau }\end{array}$ & Hoch- und Tiefbau & Bauwesen & & Bauwesen & \\
\hline 4531 & Elektroinstallation & Bauinstallation & Bauwesen & & Bauwesen & \\
\hline 4532 & $\begin{array}{l}\text { Wärme- , Kälte- , Schall- } \\
\text { und Branddämmung }\end{array}$ & Bauinstallation & Bauwesen & & Bauwesen & \\
\hline 4533 & $\begin{array}{l}\text { Gas- , Wasser-, Heizungs- u. } \\
\text { Lüftungsinstall. }\end{array}$ & Bauinstallation & Bauwesen & & Bauwesen & \\
\hline 4534 & Sonstige Bauinstallation & Bauinstallation & Bauwesen & & Bauwesen & \\
\hline 4541 & $\begin{array}{l}\text { Stukkaturgewerbe, Gipserei } \\
\text { u. Verputzerei }\end{array}$ & Ausbau- u. Bauhilfsgewerbe & Bauwesen & & Bauwesen & \\
\hline 4542 & $\begin{array}{l}\text { Bautischlerei und } \\
\text { Bauschlosserei }\end{array}$ & Ausbau- u. Bauhilfsgewerbe & Bauwesen & & Bauwesen & \\
\hline 4543 & $\begin{array}{l}\text { Fußboden-, Fliesen- u. Plat- } \\
\text { tenlegerei }\end{array}$ & Ausbau- u. Bauhilfsgewerbe & Bauwesen & & Bauwesen & \\
\hline 4544 & Malerei und Anstreicherei & Ausbau- u. Bauhilfsgewerbe & Bauwesen & & Bauwesen & \\
\hline 4545 & $\begin{array}{l}\text { Sonstiges Ausbau- und } \\
\text { Bauhilfsgewerbe }\end{array}$ & Ausbau- u. Bauhilfsgewerbe & Bauwesen & & Bauwesen & \\
\hline 4550 & $\begin{array}{l}\text { Vermietung v. Baumaschi- } \\
\text { nen mit Personal }\end{array}$ & $\begin{array}{l}\text { Vermietung v. Baumaschi- } \\
\text { nen mit Personal }\end{array}$ & Bauwesen & & Bauwesen & \\
\hline 5010 & Handel mit Kraftwagen & Handel mit Kraftwagen & $\begin{array}{l}\text { Kraftfahrzeughandel } \\
\text { Reparatur }\end{array}$ & u. & $\begin{array}{l}\text { Handel (Rep. v. } \\
\text { brauchsgütern) }\end{array}$ & KFZ u. Ge- \\
\hline 5020 & $\begin{array}{l}\text { Instandhaltung und } \\
\text { Reparatur von Kraftwa- } \\
\text { gen }\end{array}$ & $\begin{array}{l}\text { Instandhaltung und } \\
\text { Reparatur von Kraftwa- } \\
\text { gen }\end{array}$ & $\begin{array}{l}\text { Kraftfahrzeughandel } \\
\text { Reparatur }\end{array}$ & u. & $\begin{array}{l}\text { Handel (Rep. v. } \\
\text { brauchsgütern) }\end{array}$ & KFZ u. Ge- \\
\hline 5030 & $\begin{array}{l}\text { Handel mit Kraftwagenteilen } \\
\text { und Zubehör }\end{array}$ & $\begin{array}{l}\text { Handel mit Kraftwagenteilen } \\
\text { und Zubehör }\end{array}$ & $\begin{array}{l}\text { Kraftfahrzeughandel } \\
\text { Reparatur }\end{array}$ & u. & $\begin{array}{l}\text { Handel (Rep. v. } \\
\text { brauchsgütern) }\end{array}$ & KFZ u. Ge- \\
\hline 5040 & $\begin{array}{l}\text { Handel mit Krafträdern, } \\
\text { Teilen und Zubehör }\end{array}$ & $\begin{array}{l}\text { Handel mit Krafträdern, } \\
\text { Teilen und Zubehör }\end{array}$ & $\begin{array}{l}\text { Kraftfahrzeughandel } \\
\text { Reparatur }\end{array}$ & u. & $\begin{array}{l}\text { Handel (Rep. v. } \\
\text { brauchsgütern) }\end{array}$ & KFZ u. Ge- \\
\hline 5050 & Tankstellen & Tankstellen & $\begin{array}{l}\text { Kraftfahrzeughandel } \\
\text { Reparatur }\end{array}$ & u. & $\begin{array}{l}\text { Handel (Rep. v. } \\
\text { brauchsgütern) }\end{array}$ & KFZ u. Ge- \\
\hline 5111 & $\begin{array}{l}\text { Handelsvermittlung von } \\
\text { landwirtschaftlichen }\end{array}$ & Handelsvermittlung & $\begin{array}{l}\text { Handelsverm. u. G } \\
\text { del (o. Handel m. Kf }\end{array}$ & & $\begin{array}{l}\text { Handel (Rep. v. } \\
\text { brauchsgütern) }\end{array}$ & KFZ u. Ge- \\
\hline
\end{tabular}




\begin{tabular}{|c|c|c|c|c|}
\hline code & $\begin{array}{l}\text { industry } \\
\text { (very narrow) }\end{array}$ & $\begin{array}{l}\text { industry } \\
\text { (narrow) }\end{array}$ & $\begin{array}{l}\text { industry } \\
\text { (broad) }\end{array}$ & $\begin{array}{l}\text { industry } \\
\text { (very broad) }\end{array}$ \\
\hline 5112 & $\begin{array}{l}\text { Handelsvermittlung } \\
\text { Brennstoffen, Erzen, }\end{array}$ & Handelsvermittlung & $\begin{array}{l}\text { Handelsverm. u. Großhan- } \\
\text { del (o. Handel m. Kfz) }\end{array}$ & $\begin{array}{l}\text { Handel (Rep. v. KFZ u. Ge- } \\
\text { brauchsgütern) }\end{array}$ \\
\hline 5113 & $\begin{array}{l}\text { Handelsvermittlung } \\
\text { Holz, Baustoffen und }\end{array}$ & Handelsvermittlung & $\begin{array}{l}\text { Handelsverm. u. Großhan- } \\
\text { del (o. Handel m. Kfz) }\end{array}$ & $\begin{array}{l}\text { Handel (Rep. v. KFZ u. Ge- } \\
\text { brauchsgütern) }\end{array}$ \\
\hline 5114 & $\begin{array}{l}\text { Handelsvermittlung } \\
\text { Maschinen, technisch }\end{array}$ & Handelsvermittlung & $\begin{array}{l}\text { Handelsverm. u. Großhan- } \\
\text { del (o. Handel m. Kfz) }\end{array}$ & $\begin{array}{l}\text { Handel (Rep. v. KFZ u. Ge- } \\
\text { brauchsgütern) }\end{array}$ \\
\hline 5115 & $\begin{array}{l}\text { Handelsvermittlung } \\
\text { Möbeln, Einrichtungs }\end{array}$ & Handelsvermittlung & $\begin{array}{l}\text { Handelsverm. u. Großhan- } \\
\text { del (o. Handel m. Kfz) }\end{array}$ & $\begin{array}{l}\text { Handel (Rep. v. KFZ u. Ge- } \\
\text { brauchsgütern) }\end{array}$ \\
\hline 5116 & $\begin{array}{l}\text { Handelsvermittlung } \\
\text { Textilien, Bekleidun }\end{array}$ & Handelsvermittlung & $\begin{array}{l}\text { Handelsverm. u. Großhan- } \\
\text { del (o. Handel m. Kfz) }\end{array}$ & $\begin{array}{l}\text { Handel (Rep. v. KFZ u. Ge- } \\
\text { brauchsgütern) }\end{array}$ \\
\hline 5117 & $\begin{array}{l}\text { Handelsvermittlung } \\
\text { Nahrungsmitteln, Get }\end{array}$ & Handelsvermittlung & $\begin{array}{l}\text { Handelsverm. u. Großhan- } \\
\text { del (o. Handel m. Kfz) }\end{array}$ & $\begin{array}{l}\text { Handel (Rep. v. KFZ u. Ge- } \\
\text { brauchsgütern) }\end{array}$ \\
\hline 5118 & $\begin{array}{l}\text { Handelsvermittlung } \\
\text { Waren a. n. g. }\end{array}$ & Handelsvermittlung & $\begin{array}{l}\text { Handelsverm. u. Großhan- } \\
\text { del (o. Handel m. Kfz) }\end{array}$ & $\begin{array}{l}\text { Handel (Rep. v. KFZ u. Ge- } \\
\text { brauchsgütern) }\end{array}$ \\
\hline 5119 & $\begin{array}{l}\text { Handelsvermittlung ohne } \\
\text { ausgeprägten Schwer }\end{array}$ & Handelsvermittlung & $\begin{array}{l}\text { Handelsverm. u. Großhan- } \\
\text { del (o. Handel m. Kfz) }\end{array}$ & $\begin{array}{l}\text { Handel (Rep. v. KFZ u. Ge- } \\
\text { brauchsgütern) }\end{array}$ \\
\hline 5121 & $\begin{array}{l}\text { GH mit Getreide, Saaten } \\
\text { und Futterm }\end{array}$ & $\begin{array}{l}\text { GH mit landwirt. Grund- } \\
\text { stoffen u. lebenden Tieren }\end{array}$ & $\begin{array}{l}\text { Handelsverm. u. Großhan- } \\
\text { del (o. Handel m. Kfz) }\end{array}$ & $\begin{array}{l}\text { Handel (Rep. v. KFZ u. Ge- } \\
\text { brauchsgütern) }\end{array}$ \\
\hline 5122 & $\begin{array}{l}\text { GH mit Blumen und } \\
\text { Pflanzen }\end{array}$ & $\begin{array}{l}\text { GH mit landwirt. Grund- } \\
\text { stoffen u. lebenden Tieren }\end{array}$ & $\begin{array}{l}\text { Handelsverm. u. Großhan- } \\
\text { del (o. Handel m. Kfz) }\end{array}$ & $\begin{array}{l}\text { Handel (Rep. v. KFZ u. Ge- } \\
\text { brauchsgütern) }\end{array}$ \\
\hline 5123 & GH mit lebenden Tieren & $\begin{array}{l}\text { GH mit landwirt. Grund- } \\
\text { stoffen u. lebenden Tieren }\end{array}$ & $\begin{array}{l}\text { Handelsverm. u. Großhan- } \\
\text { del (o. Handel m. Kfz) }\end{array}$ & $\begin{array}{l}\text { Handel (Rep. v. KFZ u. Ge- } \\
\text { brauchsgütern) }\end{array}$ \\
\hline 5124 & $\begin{array}{l}\text { GH mit Häuten, Fellen und } \\
\text { Leder }\end{array}$ & $\begin{array}{l}\text { GH mit landwirt. Grund- } \\
\text { stoffen u. lebenden Tieren }\end{array}$ & $\begin{array}{l}\text { Handelsverm. u. Großhan- } \\
\text { del (o. Handel m. Kfz) }\end{array}$ & $\begin{array}{l}\text { Handel (Rep. v. KFZ u. Ge- } \\
\text { brauchsgütern) }\end{array}$ \\
\hline 5125 & GH mit Rohtabak & $\begin{array}{l}\text { GH mit landwirt. Grund- } \\
\text { stoffen u. lebenden Tieren }\end{array}$ & $\begin{array}{l}\text { Handelsverm. u. Großhan- } \\
\text { del (o. Handel m. Kfz) }\end{array}$ & $\begin{array}{l}\text { Handel (Rep. v. KFZ u. Ge- } \\
\text { brauchsgütern) }\end{array}$ \\
\hline 5131 & $\begin{array}{l}\text { GH mit Obst, Gemüse und } \\
\text { Kartoffeln }\end{array}$ & $\begin{array}{l}\text { GH mit Nahrungs- u. } \\
\text { Genußmitteln, Getränke, } \\
\text { Tabakw. }\end{array}$ & $\begin{array}{l}\text { Handelsverm. u. Großhan- } \\
\text { del (o. Handel m. Kfz) }\end{array}$ & $\begin{array}{l}\text { Handel (Rep. v. KFZ u. Ge- } \\
\text { brauchsgütern) }\end{array}$ \\
\hline 5132 & $\begin{array}{l}\text { GH mit Fleisch, Fleis- } \\
\text { chwaren, Geflü }\end{array}$ & $\begin{array}{l}\text { GH mit Nahrungs- u. } \\
\text { Genußmitteln, Getränke, } \\
\text { Tabakw. }\end{array}$ & $\begin{array}{l}\text { Handelsverm. u. Großhan- } \\
\text { del (o. Handel m. Kfz) }\end{array}$ & $\begin{array}{l}\text { Handel (Rep. v. KFZ u. Ge- } \\
\text { brauchsgütern) }\end{array}$ \\
\hline 5133 & $\begin{array}{l}\text { GH mit Milch, Milcherzeug- } \\
\text { nissen, Ei }\end{array}$ & $\begin{array}{l}\text { GH mit Nahrungs- u. } \\
\text { Genußmitteln, Getränke, } \\
\text { Tabakw. }\end{array}$ & $\begin{array}{l}\text { Handelsverm. u. Großhan- } \\
\text { del (o. Handel m. Kfz) }\end{array}$ & $\begin{array}{l}\text { Handel (Rep. v. KFZ u. Ge- } \\
\text { brauchsgütern) }\end{array}$ \\
\hline 5134 & GH mit Getränken & $\begin{array}{l}\text { GH mit Nahrungs- u. } \\
\text { Genußmitteln, Getränke, } \\
\text { Tabakw. }\end{array}$ & $\begin{array}{l}\text { Handelsverm. u. Großhan- } \\
\text { del (o. Handel m. Kfz) }\end{array}$ & $\begin{array}{l}\text { Handel (Rep. v. KFZ u. Ge- } \\
\text { brauchsgütern) }\end{array}$ \\
\hline 5135 & GH mit Tabakwaren & $\begin{array}{l}\text { GH mit Nahrungs- u. } \\
\text { Genußmitteln, Getränke, } \\
\text { Tabakw. }\end{array}$ & $\begin{array}{l}\text { Handelsverm. u. Großhan- } \\
\text { del (o. Handel m. Kfz) }\end{array}$ & $\begin{array}{l}\text { Handel (Rep. v. KFZ u. Ge- } \\
\text { brauchsgütern) }\end{array}$ \\
\hline 5136 & $\begin{array}{l}\text { GH mit Zucker und } \\
\text { Süßwaren }\end{array}$ & $\begin{array}{l}\text { GH mit Nahrungs- u. } \\
\text { Genußmitteln, Getränke, } \\
\text { Tabakw. }\end{array}$ & $\begin{array}{l}\text { Handelsverm. u. Großhan- } \\
\text { del (o. Handel m. Kfz) }\end{array}$ & $\begin{array}{l}\text { Handel (Rep. v. KFZ u. Ge- } \\
\text { brauchsgütern) }\end{array}$ \\
\hline 5137 & $\begin{array}{l}\text { GH mit Kaffee, Tee, Kakao } \\
\text { und Gewür }\end{array}$ & $\begin{array}{l}\text { GH mit Nahrungs- u. } \\
\text { Genußmitteln, Getränke, } \\
\text { Tabakw. }\end{array}$ & $\begin{array}{l}\text { Handelsverm. u. Großhan- } \\
\text { del (o. Handel m. Kfz) }\end{array}$ & $\begin{array}{l}\text { Handel (Rep. v. KFZ u. Ge- } \\
\text { brauchsgütern) }\end{array}$ \\
\hline 5138 & $\begin{array}{l}\text { GH mit sonstigen Nahrungs- } \\
\text { und Genu }\end{array}$ & $\begin{array}{l}\text { GH mit Nahrungs- u. } \\
\text { Genußmitteln, Getränke, } \\
\text { Tabakw. }\end{array}$ & $\begin{array}{l}\text { Handelsverm. u. Großhan- } \\
\text { del (o. Handel m. Kfz) }\end{array}$ & $\begin{array}{l}\text { Handel (Rep. v. KFZ u. Ge- } \\
\text { brauchsgütern) }\end{array}$ \\
\hline 5139 & $\begin{array}{l}\text { GH mit Nahrungs- und } \\
\text { Genußmitteln, }\end{array}$ & $\begin{array}{l}\text { GH mit Nahrungs- u. } \\
\text { Genußmitteln, Getränke, } \\
\text { Tabakw. }\end{array}$ & $\begin{array}{l}\text { Handelsverm. u. Großhan- } \\
\text { del (o. Handel m. Kfz) }\end{array}$ & $\begin{array}{l}\text { Handel (Rep. v. KFZ u. Ge- } \\
\text { brauchsgütern) }\end{array}$ \\
\hline 5141 & GH mit Textilien & $\begin{array}{l}\text { GH mit Gebrauchs- u. Ver- } \\
\text { brauchsgütern }\end{array}$ & $\begin{array}{l}\text { Handelsverm. u. Großhan- } \\
\text { del (o. Handel m. Kfz) }\end{array}$ & $\begin{array}{l}\text { Handel (Rep. v. KFZ u. Ge- } \\
\text { brauchsgütern) }\end{array}$ \\
\hline 5142 & $\begin{array}{l}\text { GH mit Bekleidung und } \\
\text { Schuhen }\end{array}$ & $\begin{array}{l}\text { GH mit Gebrauchs- u. Ver- } \\
\text { brauchsgütern }\end{array}$ & $\begin{array}{l}\text { Handelsverm. u. Großhan- } \\
\text { del (o. Handel m. Kfz) }\end{array}$ & $\begin{array}{l}\text { Handel (Rep. v. KFZ u. Ge- } \\
\text { brauchsgütern) }\end{array}$ \\
\hline 5143 & $\begin{array}{l}\text { GH mit elektrischen } \\
\text { Haushaltsgeräte }\end{array}$ & $\begin{array}{l}\text { GH mit Gebrauchs- u. Ver- } \\
\text { brauchsgütern }\end{array}$ & $\begin{array}{l}\text { Handelsverm. u. Großhan- } \\
\text { del (o. Handel m. Kfz) }\end{array}$ & $\begin{array}{l}\text { Handel (Rep. v. KFZ u. Ge- } \\
\text { brauchsgütern) }\end{array}$ \\
\hline 5144 & $\begin{array}{l}\text { GH mit Haushaltswaren aus } \\
\text { Metall, } \mathrm{k}\end{array}$ & $\begin{array}{l}\text { GH mit Gebrauchs- u. Ver- } \\
\text { brauchsgütern }\end{array}$ & $\begin{array}{l}\text { Handelsverm. u. Großhan- } \\
\text { del (o. Handel m. Kfz) }\end{array}$ & $\begin{array}{l}\text { Handel (Rep. v. KFZ u. Ge- } \\
\text { brauchsgütern) }\end{array}$ \\
\hline 5145 & $\begin{array}{l}\text { GH mit kosmetischen } \\
\text { Erzeugnissen un }\end{array}$ & $\begin{array}{l}\text { GH mit Gebrauchs- u. Ver- } \\
\text { brauchsgütern }\end{array}$ & $\begin{array}{l}\text { Handelsverm. u. Großhan- } \\
\text { del (o. Handel m. Kfz) }\end{array}$ & $\begin{array}{l}\text { Handel (Rep. v. KFZ u. Ge- } \\
\text { brauchsgütern) }\end{array}$ \\
\hline
\end{tabular}




\begin{tabular}{|c|c|c|c|c|}
\hline code & $\begin{array}{l}\text { industry } \\
\text { (very narrow) }\end{array}$ & $\begin{array}{l}\text { industry } \\
\text { (narrow) }\end{array}$ & $\begin{array}{l}\text { industry } \\
\text { (broad) }\end{array}$ & $\begin{array}{l}\text { industry } \\
\text { (very broad) }\end{array}$ \\
\hline 5146 & $\begin{array}{l}\text { GH mit pharmazeutischen } \\
\text { Erzeugnisse }\end{array}$ & $\begin{array}{l}\text { GH mit Gebrauchs- u. Ver- } \\
\text { brauchsgütern }\end{array}$ & $\begin{array}{l}\text { Handelsverm. u. Großhan- } \\
\text { del (o. Handel m. Kfz) }\end{array}$ & $\begin{array}{l}\text { Handel (Rep. v. KFZ u. Ge- } \\
\text { brauchsgütern) }\end{array}$ \\
\hline 5147 & $\begin{array}{l}\text { GH mit sonstigen } \\
\text { Gebrauchs- und Ver }\end{array}$ & $\begin{array}{l}\text { GH mit Gebrauchs- } u \text {. Ver- } \\
\text { brauchsgütern }\end{array}$ & $\begin{array}{l}\text { Handelsverm. u. Großhan- } \\
\text { del (o. Handel m. Kfz) }\end{array}$ & $\begin{array}{l}\text { Handel (Rep. v. KFZ u. Ge- } \\
\text { brauchsgütern) }\end{array}$ \\
\hline 5151 & $\begin{array}{l}\text { GH mit festen Brennstoffen } \\
\text { und Mine }\end{array}$ & $\begin{array}{l}\text { GH mit Rohstoffen, Halb- } \\
\text { waren, Altmaterial }\end{array}$ & $\begin{array}{l}\text { Handelsverm. u. Großhan- } \\
\text { del (o. Handel m. Kfz) }\end{array}$ & $\begin{array}{l}\text { Handel (Rep. v. KFZ u. Ge- } \\
\text { brauchsgütern) }\end{array}$ \\
\hline 5152 & $\begin{array}{l}\text { GH mit Erzen, Eisen, Stahl, } \\
\text { NE- Meta }\end{array}$ & $\begin{array}{l}\text { GH mit Rohstoffen, Halb- } \\
\text { waren, Altmaterial }\end{array}$ & $\begin{array}{l}\text { Handelsverm. u. Großhan- } \\
\text { del (o. Handel m. Kfz) }\end{array}$ & $\begin{array}{l}\text { Handel (Rep. v. KFZ u. Ge- } \\
\text { brauchsgütern) }\end{array}$ \\
\hline 5153 & $\begin{array}{l}\text { GH mit Holz, Baustoffen, } \\
\text { Anstrichmi }\end{array}$ & $\begin{array}{l}\text { GH mit Rohstoffen, Halb- } \\
\text { waren, Altmaterial }\end{array}$ & $\begin{array}{l}\text { Handelsverm. u. Großhan- } \\
\text { del (o. Handel m. Kfz) }\end{array}$ & $\begin{array}{l}\text { Handel (Rep. v. KFZ u. Ge- } \\
\text { brauchsgütern) }\end{array}$ \\
\hline 5154 & $\begin{array}{l}\text { GH mit Bauelementen aus } \\
\text { Metall sowi }\end{array}$ & $\begin{array}{l}\text { GH mit Rohstoffen, Halb- } \\
\text { waren, Altmaterial }\end{array}$ & $\begin{array}{l}\text { Handelsverm. u. Großhan- } \\
\text { del (o. Handel m. Kfz) }\end{array}$ & $\begin{array}{l}\text { Handel (Rep. v. KFZ u. Ge- } \\
\text { brauchsgütern) }\end{array}$ \\
\hline 5155 & $\begin{array}{l}\text { GH mit chemischen Erzeug- } \\
\text { nissen }\end{array}$ & $\begin{array}{l}\text { GH mit Rohstoffen, Halb- } \\
\text { waren, Altmaterial }\end{array}$ & $\begin{array}{l}\text { Handelsverm. u. Großhan- } \\
\text { del (o. Handel m. Kfz) }\end{array}$ & $\begin{array}{l}\text { Handel (Rep. v. KFZ u. Ge- } \\
\text { brauchsgütern) }\end{array}$ \\
\hline 5156 & GH mit sonstigen Halbwaren & $\begin{array}{l}\text { GH mit Rohstoffen, Halb- } \\
\text { waren, Altmaterial }\end{array}$ & $\begin{array}{l}\text { Handelsverm. u. Großhan- } \\
\text { del (o. Handel m. Kfz) }\end{array}$ & $\begin{array}{l}\text { Handel (Rep. v. KFZ u. Ge- } \\
\text { brauchsgütern) }\end{array}$ \\
\hline 5157 & $\begin{array}{l}\text { GH mit Altmaterial und } \\
\text { Reststoffen }\end{array}$ & $\begin{array}{l}\text { GH mit Rohstoffen, Halb- } \\
\text { waren, Altmaterial }\end{array}$ & $\begin{array}{l}\text { Handelsverm. u. Großhan- } \\
\text { del (o. Handel m. Kfz) }\end{array}$ & $\begin{array}{l}\text { Handel (Rep. v. KFZ u. Ge- } \\
\text { brauchsgütern) }\end{array}$ \\
\hline 5161 & $\begin{array}{l}\text { GH mit Werkzeugmaschinen } \\
\text { (bis } 7 / 04 \text { ) }\end{array}$ & $\begin{array}{l}\text { GH mit Masch. } \\
\text { Ausrüstungen u. Zubehör }\end{array}$ & $\begin{array}{l}\text { Handelsverm. u. Großhan- } \\
\text { del (o. Handel m. Kfz) }\end{array}$ & $\begin{array}{l}\text { Handel (Rep. v. KFZ u. Ge- } \\
\text { brauchsgütern) }\end{array}$ \\
\hline 5162 & $\begin{array}{l}\text { GH mit Baumaschinen (bis } \\
7 / 04 \text { ) }\end{array}$ & $\begin{array}{l}\text { GH mit Masch. } \\
\text { Ausrüstungen u. Zubehör }\end{array}$ & $\begin{array}{l}\text { Handelsverm. u. Großhan- } \\
\text { del (o. Handel m. Kfz) }\end{array}$ & $\begin{array}{l}\text { Handel (Rep. v. KFZ u. Ge- } \\
\text { brauchsgütern) }\end{array}$ \\
\hline 5163 & $\begin{array}{l}\text { GH mit Textil- , Näh- u. } \\
\text { Strickmaschinen (bis } 7 / 04 \text { ) }\end{array}$ & $\begin{array}{l}\text { GH mit Masch. } \\
\text { Ausrüstungen u. Zubehör }\end{array}$ & $\begin{array}{l}\text { Handelsverm. u. Großhan- } \\
\text { del (o. Handel m. Kfz) }\end{array}$ & $\begin{array}{l}\text { Handel (Rep. v. KFZ u. Ge- } \\
\text { brauchsgütern) }\end{array}$ \\
\hline 5164 & $\begin{array}{l}\text { GH mit Büromaschinen u. - } \\
\text { einrichtungen (bis } 7 / 04 \text { ) }\end{array}$ & $\begin{array}{l}\text { GH mit Masch. } \\
\text { Ausrüstungen u. Zubehör }\end{array}$ & $\begin{array}{l}\text { Handelsverm. u. Großhan- } \\
\text { del (o. Handel m. Kfz) }\end{array}$ & $\begin{array}{l}\text { Handel (Rep. v. KFZ u. Ge- } \\
\text { brauchsgütern) }\end{array}$ \\
\hline 5165 & $\begin{array}{l}\text { GH mit sonst. Maschinen u. } \\
\text { Ausrüstungen (bis } 7 / 04 \text { ) }\end{array}$ & $\begin{array}{l}\text { GH mit Masch. } \\
\text { Ausrüstungen u. Zubehör }\end{array}$ & $\begin{array}{l}\text { Handelsverm. u. Großhan- } \\
\text { del (o. Handel m. Kfz) }\end{array}$ & $\begin{array}{l}\text { Handel (Rep. v. KFZ u. Ge- } \\
\text { brauchsgütern) }\end{array}$ \\
\hline 5166 & $\begin{array}{l}\text { GH mit landwirtschaftlichen } \\
\text { Maschinen (bis } 7 / 04 \text { ) }\end{array}$ & $\begin{array}{l}\text { GH mit Masch. } \\
\text { Ausrüstungen u. Zubehör }\end{array}$ & $\begin{array}{l}\text { Handelsverm. u. Großhan- } \\
\text { del (o. Handel m. Kfz) }\end{array}$ & $\begin{array}{l}\text { Handel (Rep. v. KFZ u. Ge- } \\
\text { brauchsgütern) }\end{array}$ \\
\hline 5170 & $\begin{array}{l}\text { Sonstiger Großhandel (bis } \\
7 / 04)\end{array}$ & Sonstiger Großhandel & $\begin{array}{l}\text { Handelsverm. u. Großhan- } \\
\text { del (o. Handel m. Kfz) }\end{array}$ & $\begin{array}{l}\text { Handel (Rep. v. KFZ u. Ge- } \\
\text { brauchsgütern) }\end{array}$ \\
\hline 5181 & GH mit Werkzeugmaschinen & $\begin{array}{l}\text { GH mit Masch. } \\
\text { Ausrüstungen u. Zubehör }\end{array}$ & $\begin{array}{l}\text { Handelsverm. u. Großhan- } \\
\text { del (o. Handel m. Kfz) }\end{array}$ & $\begin{array}{l}\text { Handel (Rep. v. KFZ u. Ge- } \\
\text { brauchsgütern) }\end{array}$ \\
\hline 5182 & GH mit Baumaschinen & $\begin{array}{l}\text { GH mit Masch. } \\
\text { Ausrüstungen u. Zubehör }\end{array}$ & $\begin{array}{l}\text { Handelsverm. u. Großhan- } \\
\text { del (o. Handel m. Kfz) }\end{array}$ & $\begin{array}{l}\text { Handel (Rep. v. KFZ u. Ge- } \\
\text { brauchsgütern) }\end{array}$ \\
\hline 5183 & $\begin{array}{l}\text { GH mit Textil- , Näh- u. } \\
\text { Strickmaschinen }\end{array}$ & $\begin{array}{l}\text { GH mit Masch. } \\
\text { Ausrüstungen u. Zubehör }\end{array}$ & $\begin{array}{l}\text { Handelsverm. u. Großhan- } \\
\text { del (o. Handel m. Kfz) }\end{array}$ & $\begin{array}{l}\text { Handel (Rep. v. KFZ u. Ge- } \\
\text { brauchsgütern) }\end{array}$ \\
\hline 5184 & $\begin{array}{l}\text { GH mit Datenverarbeitungs- } \\
\text { geräten u. peripheren Ein- } \\
\text { heiten }\end{array}$ & $\begin{array}{l}\text { GH mit Masch. } \\
\text { Ausrüstungen u. Zubehör }\end{array}$ & $\begin{array}{l}\text { Handelsverm. u. Großhan- } \\
\text { del (o. Handel m. Kfz) }\end{array}$ & $\begin{array}{l}\text { Handel (Rep. v. KFZ u. Ge- } \\
\text { brauchsgütern) }\end{array}$ \\
\hline 5185 & $\begin{array}{l}\text { GH mit } \\
\text { Büromaschinen }\end{array}$ & $\begin{array}{l}\text { GH mit Masch. } \\
\text { Ausrüstungen u. Zubehör }\end{array}$ & $\begin{array}{l}\text { Handelsverm. u. Großhan- } \\
\text { del (o. Handel m. Kfz) }\end{array}$ & $\begin{array}{l}\text { Handel (Rep. v. KFZ u. Ge- } \\
\text { brauchsgütern) }\end{array}$ \\
\hline 5186 & $\begin{array}{l}\text { GH mit elektron. Bauele- } \\
\text { menten }\end{array}$ & $\begin{array}{l}\text { GH mit Masch. } \\
\text { Ausrüstungen u. Zubehör }\end{array}$ & $\begin{array}{l}\text { Handelsverm. u. Großhan- } \\
\text { del (o. Handel m. Kfz) }\end{array}$ & $\begin{array}{l}\text { Handel (Rep. v. KFZ u. Ge- } \\
\text { brauchsgütern) }\end{array}$ \\
\hline 5187 & $\begin{array}{l}\text { GH mit sonst. Maschinen, } \\
\text { Ausrüstungen u. Zubehör }\end{array}$ & $\begin{array}{l}\text { GH mit Masch. } \\
\text { Ausrüstungen u. Zubehör }\end{array}$ & $\begin{array}{l}\text { Handelsverm. u. Großhan- } \\
\text { del (o. Handel m. Kfz) }\end{array}$ & $\begin{array}{l}\text { Handel (Rep. v. KFZ u. Ge- } \\
\text { brauchsgütern) }\end{array}$ \\
\hline 5188 & $\begin{array}{l}\text { GH mit landw. Maschinen u. } \\
\text { Geräten }\end{array}$ & $\begin{array}{l}\text { GH mit Masch. } \\
\text { Ausrüstungen u. Zubehör }\end{array}$ & $\begin{array}{l}\text { Handelsverm. u. Großhan- } \\
\text { del (o. Handel m. Kfz) }\end{array}$ & $\begin{array}{l}\text { Handel (Rep. v. KFZ u. Ge- } \\
\text { brauchsgütern) }\end{array}$ \\
\hline 5190 & Sonstiger Großhandel & Sonstiger Großhandel & $\begin{array}{l}\text { Handelsverm. u. Großhan- } \\
\text { del (o. Handel m. Kfz) }\end{array}$ & $\begin{array}{l}\text { Handel (Rep. v. KFZ u. Ge- } \\
\text { brauchsgütern) }\end{array}$ \\
\hline 5211 & $\begin{array}{l}\text { Einzelhandel mit Waren ver- } \\
\text { schiedener Art }\end{array}$ & $\begin{array}{l}\text { EH mit Waren versch. Art } \\
\text { (in Verkaufsräumen) }\end{array}$ & $\begin{array}{l}\text { Einzelhandel (o. Handel } \mathrm{m} \text {. } \\
\text { Kfz) }\end{array}$ & $\begin{array}{l}\text { Handel (Rep. v. KFZ u. Ge- } \\
\text { brauchsgütern) }\end{array}$ \\
\hline 5212 & $\begin{array}{l}\text { Sonstiger Einzelhandel mit } \\
\text { Waren verschiede }\end{array}$ & $\begin{array}{l}\text { EH mit Waren versch. Art } \\
\text { (in Verkaufsräumen) }\end{array}$ & $\begin{array}{l}\text { Einzelhandel (o. Handel } \mathrm{m} \text {. } \\
\text { Kfz) }\end{array}$ & $\begin{array}{l}\text { Handel (Rep. v. KFZ u. Ge- } \\
\text { brauchsgütern) }\end{array}$ \\
\hline 5221 & $\begin{array}{l}\text { Einzelhandel mit Obst, } \\
\text { Gemüse und Kartoffel }\end{array}$ & $\begin{array}{l}\text { EH mit Nahrungs- u. } \\
\text { Genussmitteln, Getränke, } \\
\text { Tabakw. }\end{array}$ & $\begin{array}{l}\text { Einzelhandel (o. Handel } \mathrm{m} \text {. } \\
\text { Kfz) }\end{array}$ & $\begin{array}{l}\text { Handel (Rep. v. KFZ u. Ge- } \\
\text { brauchsgütern) }\end{array}$ \\
\hline 5222 & $\begin{array}{l}\text { Einzelhandel mit Fleisch und } \\
\text { Fleischwaren, }\end{array}$ & $\begin{array}{l}\text { EH mit Nahrungs- u. } \\
\text { Genussmitteln, Getränke, } \\
\text { Tabakw. }\end{array}$ & $\begin{array}{l}\text { Einzelhandel (o. Handel m. } \\
\text { Kfz) }\end{array}$ & $\begin{array}{l}\text { Handel (Rep. v. KFZ u. Ge- } \\
\text { brauchsgütern) }\end{array}$ \\
\hline
\end{tabular}




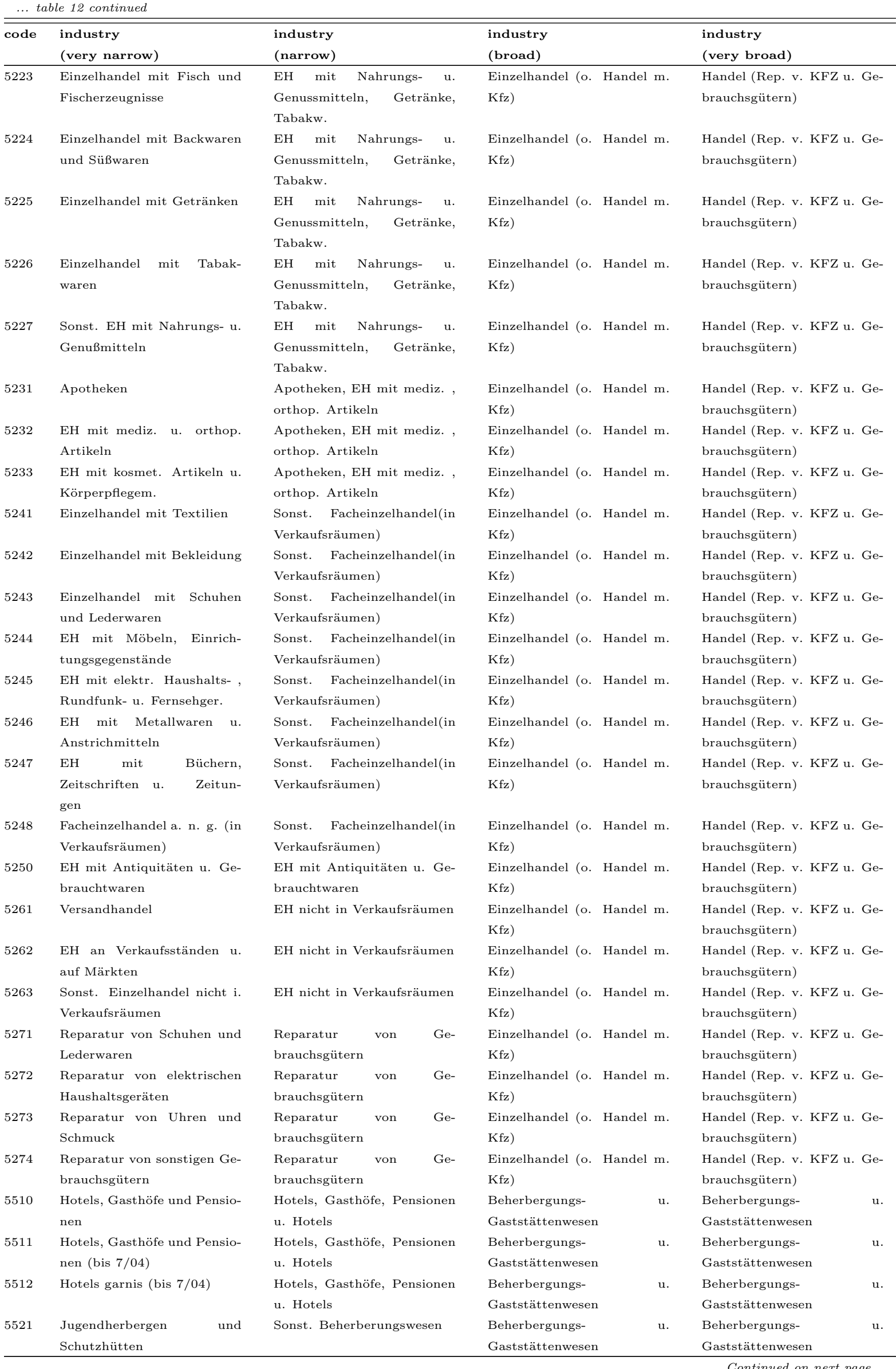









\begin{tabular}{|c|c|c|c|c|c|}
\hline code & $\begin{array}{l}\text { industry } \\
\text { (very narrow) }\end{array}$ & $\begin{array}{l}\text { industry } \\
\text { (narrow) }\end{array}$ & $\begin{array}{l}\text { industry } \\
\text { (broad) }\end{array}$ & $\begin{array}{l}\text { industry } \\
\text { (very broad) }\end{array}$ & \\
\hline 6522 & Spezialkreditinstitute & $\begin{array}{l}\text { Sonst. Finanzierungsinstitu- } \\
\text { tionen }\end{array}$ & Kreditwesen & $\begin{array}{l}\text { Kredit- und } \\
\text { sicherungswesen }\end{array}$ & Ver- \\
\hline 6523 & $\begin{array}{l}\text { Finanzierungsinstitutionen } \\
\text { a. n. g. }\end{array}$ & $\begin{array}{l}\text { Sonst. Finanzierungsinstitu- } \\
\text { tionen }\end{array}$ & Kreditwesen & $\begin{array}{l}\text { Kredit- und } \\
\text { sicherungswesen }\end{array}$ & Ver- \\
\hline 6601 & Lebensversicherung & Versicherungswesen & Versicherungswesen & $\begin{array}{l}\text { Kredit- und } \\
\text { sicherungswesen }\end{array}$ & Ver- \\
\hline 6602 & Pensionskassen & Versicherungswesen & Versicherungswesen & $\begin{array}{l}\text { Kredit- und } \\
\text { sicherungswesen }\end{array}$ & Ver- \\
\hline 6603 & $\begin{array}{l}\text { Sonstiges Versicherungswe- } \\
\text { sen }\end{array}$ & Versicherungswesen & Versicherungswesen & $\begin{array}{l}\text { Kredit- und } \\
\text { sicherungswesen }\end{array}$ & Ver- \\
\hline 6711 & $\begin{array}{l}\text { Effekten- und Warenter- } \\
\text { minbörsen }\end{array}$ & $\begin{array}{l}\text { Mit d. Kreditwesen verbun- } \\
\text { dene Tätigkeit }\end{array}$ & $\begin{array}{l}\text { Mit d. Kredit- u. Vers. We- } \\
\text { sen verbundene Tätigkeiten }\end{array}$ & $\begin{array}{l}\text { Kredit- und } \\
\text { sicherungswesen }\end{array}$ & Ver- \\
\hline 6712 & $\begin{array}{l}\text { Effektenvermittlung und - } \\
\text { verwaltung }\end{array}$ & $\begin{array}{l}\text { Mit d. Kreditwesen verbun- } \\
\text { dene Tätigkeit }\end{array}$ & $\begin{array}{l}\text { Mit d. Kredit- u. Vers. We- } \\
\text { sen verbundene Tätigkeiten }\end{array}$ & $\begin{array}{l}\text { Kredit- und } \\
\text { sicherungswesen }\end{array}$ & Ver- \\
\hline 6713 & $\begin{array}{l}\text { Sonst. mit d. Kreditwesen } \\
\text { verbundene Tätigkeit }\end{array}$ & $\begin{array}{l}\text { Mit d. Kreditwesen verbun- } \\
\text { dene Tätigkeit }\end{array}$ & $\begin{array}{l}\text { Mit d. Kredit- u. Vers. We- } \\
\text { sen verbundene Tätigkeiten }\end{array}$ & $\begin{array}{l}\text { Kredit- und } \\
\text { sicherungswesen }\end{array}$ & Ver- \\
\hline 6720 & $\begin{array}{l}\text { Mit d. Versicherungswesen } \\
\text { verbundene Tätigkeit }\end{array}$ & $\begin{array}{l}\text { Mit d. Versicherungswesen } \\
\text { verbundene Tätigkeit }\end{array}$ & $\begin{array}{l}\text { Mit d. Kredit- u. Vers. We- } \\
\text { sen verbundene Tätigkeiten }\end{array}$ & $\begin{array}{l}\text { Kredit- und } \\
\text { sicherungswesen }\end{array}$ & Ver- \\
\hline 7011 & $\begin{array}{l}\text { Erschließung } \\
\text { Grundstücken }\end{array}$ & $\begin{array}{l}\text { Erschl. , Kauf u. Verkauf v. } \\
\text { eigenen Grundst. u. Real }\end{array}$ & Realitätenwesen & $\begin{array}{l}\text { Realitäten, Rechts- } \\
\text { Wirtsch. Dienste }\end{array}$ & u. \\
\hline 7012 & $\begin{array}{l}\text { Kauf u. Verkauf v. eigenen } \\
\text { Grundst. u. Realitäten }\end{array}$ & $\begin{array}{l}\text { Erschl. , Kauf u. Verkauf v. } \\
\text { eigenen Grundst. u. Real }\end{array}$ & Realitätenwesen & $\begin{array}{l}\text { Realitäten, Rechts- } \\
\text { Wirtsch. Dienste }\end{array}$ & u. \\
\hline 7020 & $\begin{array}{l}\text { Vermiet. u. Verpacht. v. } \\
\text { eigenen Grundst. u. Re- } \\
\text { alitäten }\end{array}$ & $\begin{array}{l}\text { Vermiet. u. Verpacht. v. } \\
\text { eigenen Grundst. u. Re- } \\
\text { alität }\end{array}$ & Realitätenwesen & $\begin{array}{l}\text { Realitäten, Rechts- } \\
\text { Wirtsch. Dienste }\end{array}$ & u. \\
\hline 7031 & $\begin{array}{l}\text { Vermittlung v. } \\
\text { Grundstücken u. Realitäten }\end{array}$ & $\begin{array}{l}\text { Verm. u. Verw. v. } \\
\text { Grundstücken } \\
\text { u. Realitäten }\end{array}$ & Realitätenwesen & $\begin{array}{l}\text { Realitäten, Rechts- } \\
\text { Wirtsch. Dienste }\end{array}$ & u. \\
\hline 7032 & $\begin{array}{l}\text { Verwaltung } \\
\text { Grundstücken, } \\
\text { Hauswartung }\end{array}$ & $\begin{array}{l}\text { Verm. u. Verw. v. } \\
\text { Grundstücken } \\
\text { u. Realitäten }\end{array}$ & Realitätenwesen & $\begin{array}{l}\text { Realitäten, Rechts- } \\
\text { Wirtsch. Dienste }\end{array}$ & u. \\
\hline 7110 & $\begin{array}{l}\text { Vermietung v. Kraftwagen } \\
\text { bis } 3 \text {, 5t Gesamtgew. }\end{array}$ & $\begin{array}{l}\text { Vermietung v. Kraftwagen } \\
\text { bis } 3,5 \text { t Gesamtgew. }\end{array}$ & $\begin{array}{l}\text { Vermietung bewegl. Sachen } \\
\text { o. Bedienungspersonal }\end{array}$ & $\begin{array}{l}\text { Realitäten, Rechts- } \\
\text { Wirtsch. Dienste }\end{array}$ & u. \\
\hline 7121 & $\begin{array}{l}\text { Vermietung von Land- } \\
\text { fahrzeugen }\end{array}$ & $\begin{array}{l}\text { Vermietung v. } \quad \text { sonst. } \\
\text { Verkehrsmitteln }\end{array}$ & $\begin{array}{l}\text { Vermietung bewegl. Sachen } \\
\text { o. Bedienungspersonal }\end{array}$ & $\begin{array}{l}\text { Realitäten, Rechts- } \\
\text { Wirtsch. Dienste }\end{array}$ & u. \\
\hline 7122 & $\begin{array}{l}\text { Vermietung von Wasser- } \\
\text { fahrzeugen }\end{array}$ & $\begin{array}{l}\text { Vermietung } \quad \mathrm{v} . \\
\text { Verkehrsmitteln }\end{array}$ & $\begin{array}{l}\text { Vermietung bewegl. Sachen } \\
\text { o. Bedienungspersonal }\end{array}$ & $\begin{array}{l}\text { Realitäten, Rechts- } \\
\text { Wirtsch. Dienste }\end{array}$ & u. \\
\hline 7123 & $\begin{array}{l}\text { Vermietung von Luftfahrzeu- } \\
\text { gen }\end{array}$ & $\begin{array}{l}\text { Vermietung v. } \quad \text { sonst. } \\
\text { Verkehrsmitteln }\end{array}$ & $\begin{array}{l}\text { Vermietung bewegl. Sachen } \\
\text { o. Bedienungspersonal }\end{array}$ & $\begin{array}{l}\text { Realitäten, Rechts- } \\
\text { Wirtsch. Dienste }\end{array}$ & u. \\
\hline 7131 & $\begin{array}{l}\text { Vermietung v. land- } \\
\text { wirtschaftl. Maschinen }\end{array}$ & $\begin{array}{l}\text { Vermietung v. Maschinen } \\
\text { und Geräten }\end{array}$ & $\begin{array}{l}\text { Vermietung bewegl. Sachen } \\
\text { o. Bedienungspersonal }\end{array}$ & $\begin{array}{l}\text { Realitäten, Rechts- } \\
\text { Wirtsch. Dienste }\end{array}$ & u. \\
\hline 7132 & $\begin{array}{l}\text { Vermietung v. Baumaschi- } \\
\text { nen und - geräten }\end{array}$ & $\begin{array}{l}\text { Vermietung v. Maschinen } \\
\text { und Geräten }\end{array}$ & $\begin{array}{l}\text { Vermietung bewegl. Sachen } \\
\text { o. Bedienungspersonal }\end{array}$ & $\begin{array}{l}\text { Realitäten, Rechts- } \\
\text { Wirtsch. Dienste }\end{array}$ & u. \\
\hline 7133 & $\begin{array}{l}\text { Vermietung v. Büromasch. } \\
\text { Datenverarbeitungsger. }\end{array}$ & $\begin{array}{l}\text { Vermietung v. Maschinen } \\
\text { und Geräten }\end{array}$ & $\begin{array}{l}\text { Vermietung bewegl. Sachen } \\
\text { o. Bedienungspersonal }\end{array}$ & $\begin{array}{l}\text { Realitäten, Rechts- } \\
\text { Wirtsch. Dienste }\end{array}$ & u. \\
\hline 7134 & $\begin{array}{l}\text { Vermietung v. sonst. } \\
\text { Maschinen und Geräten }\end{array}$ & $\begin{array}{l}\text { Vermietung v. Maschinen } \\
\text { und Geräten }\end{array}$ & $\begin{array}{l}\text { Vermietung bewegl. Sachen } \\
\text { o. Bedienungspersonal }\end{array}$ & $\begin{array}{l}\text { Realitäten, Rechts- } \\
\text { Wirtsch. Dienste }\end{array}$ & u. \\
\hline 7140 & $\begin{array}{l}\text { Vermietung von Ge- } \\
\text { brauchsgütern a. n. g. }\end{array}$ & $\begin{array}{l}\text { Vermietung von Ge- } \\
\text { brauchsgütern a. n. g. }\end{array}$ & $\begin{array}{l}\text { Vermietung bewegl. Sachen } \\
\text { o. Bedienungspersonal }\end{array}$ & $\begin{array}{l}\text { Realitäten, Rechts- } \\
\text { Wirtsch. Dienste }\end{array}$ & $\mathrm{u}$. \\
\hline 7210 & Hardwareberatung & Hardwareberatung & $\begin{array}{l}\text { Datenverarbeitung } \\
\text { Datenbanken }\end{array}$ & $\begin{array}{l}\text { Realitäten, Rechts- } \\
\text { Wirtsch. Dienste }\end{array}$ & u. \\
\hline 7220 & Softwarehäuser (bis $7 / 04$ ) & Softwarehäuser & $\begin{array}{l}\text { Datenverarbeitung } \\
\text { Datenbanken }\end{array}$ & $\begin{array}{l}\text { Realitäten, Rechts- } \\
\text { Wirtsch. Dienste }\end{array}$ & u. \\
\hline 7221 & $\begin{array}{l}\text { Verlag u. Herst. von Stan- } \\
\text { dardsoftware }\end{array}$ & Softwarehäuser & $\begin{array}{l}\text { Datenverarbeitung } \\
\text { Datenbanken }\end{array}$ & $\begin{array}{l}\text { Realitäten, Rechts- } \\
\text { Wirtsch. Dienste }\end{array}$ & u. \\
\hline 7222 & $\begin{array}{l}\text { Softwareberatung } \mathrm{u} \text {. - en- } \\
\text { twicklung }\end{array}$ & Softwarehäuser & $\begin{array}{l}\text { Datenverarbeitung } \\
\text { Datenbanken }\end{array}$ & $\begin{array}{l}\text { Realitäten, Rechts- } \\
\text { Wirtsch. Dienste }\end{array}$ & u. \\
\hline 7230 & Datenverarbeitungsdienste & Datenverarbeitungsdienste & $\begin{array}{l}\text { Datenverarbeitung } \\
\text { Datenbanken }\end{array}$ & $\begin{array}{l}\text { Realitäten, Rechts- } \\
\text { Wirtsch. Dienste }\end{array}$ & $\mathrm{u}$. \\
\hline 7240 & Datenbanken & Datenbanken & $\begin{array}{l}\text { Datenverarbeitung } \\
\text { Datenbanken }\end{array}$ & $\begin{array}{l}\text { Realitäten, Rechts- } \\
\text { Wirtsch. Dienste }\end{array}$ & $\mathrm{u}$. \\
\hline 7250 & Instandhaltung & Instandhaltung & Datenverarbeitung & Realitäten, & u. \\
\hline & Reparatur & Reparatur & Datenbanken & Wirtsch. Dienste & \\
\hline & Büromaschinen & Büromaschinen & & & \\
\hline
\end{tabular}




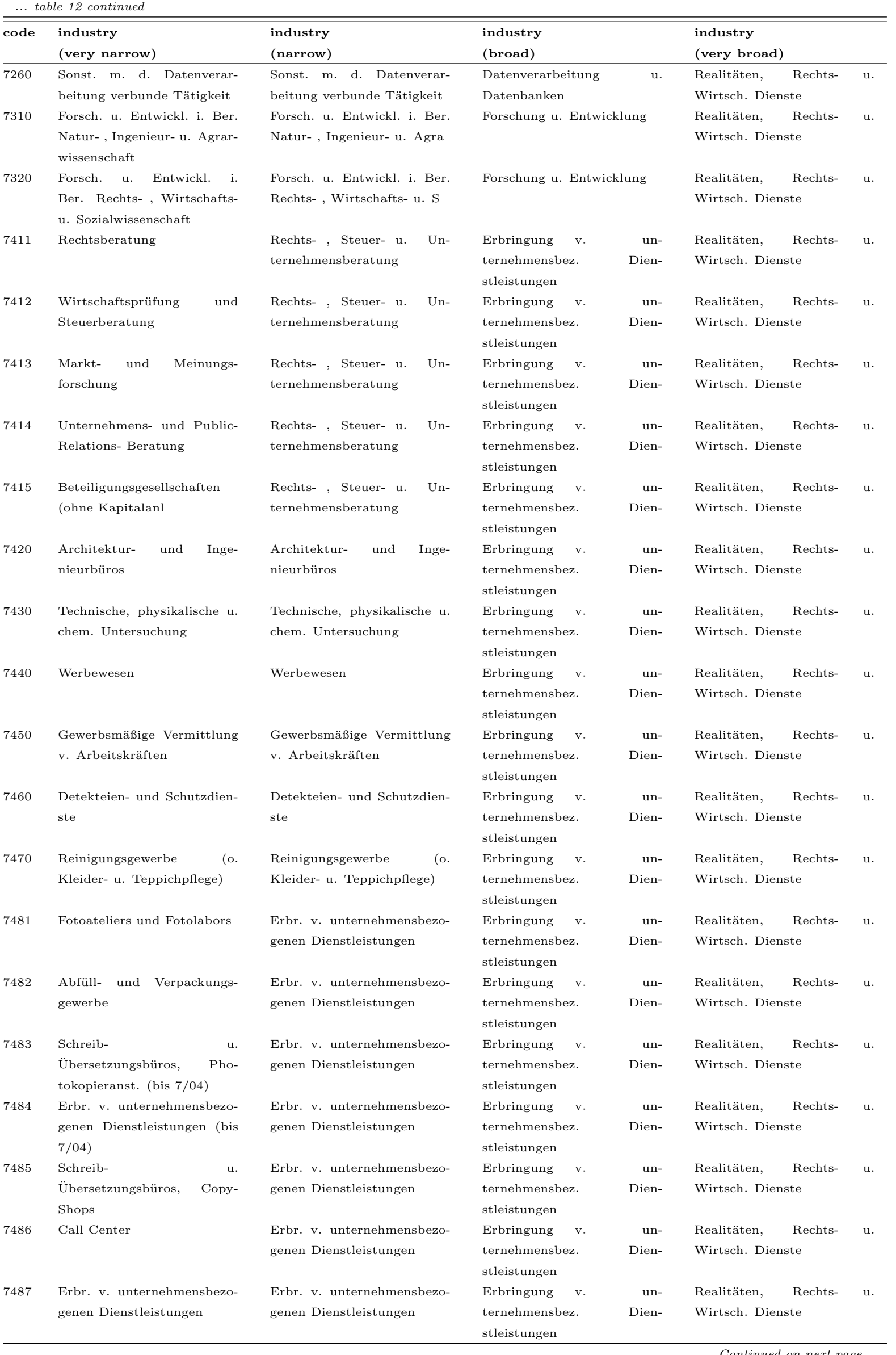




\begin{tabular}{|c|c|c|c|c|}
\hline code & $\begin{array}{l}\text { industry } \\
\text { (very narrow) }\end{array}$ & $\begin{array}{l}\text { industry } \\
\text { (narrow) }\end{array}$ & $\begin{array}{l}\text { industry } \\
\text { (broad) }\end{array}$ & $\begin{array}{l}\text { industry } \\
\text { (very broad) }\end{array}$ \\
\hline 7511 & $\begin{array}{l}\text { Allgemeine öffentliche Ver- } \\
\text { waltung }\end{array}$ & Öffentliche Verwaltung & $\begin{array}{l}\text { Öffentl. Verwaltung, } \\
\text { Sozialvers. , Landesverteidi- } \\
\text { gung }\end{array}$ & $\begin{array}{l}\text { Öffentl. Verwaltung, Lan- } \\
\text { desvert. , Sozialvers. }\end{array}$ \\
\hline 7512 & $\begin{array}{l}\text { Öffentl. Verwaltung a. d. } \\
\text { Gebieten Gesundh. } \quad-\mathrm{u} . \\
\text { Sozialwesen }\end{array}$ & Öffentliche Verwaltung & $\begin{array}{l}\text { Öffentl. Verwaltung, } \\
\text { Sozialvers. , Landesverteidi- } \\
\text { gung }\end{array}$ & $\begin{array}{l}\text { Öffentl. Verwaltung, Lan- } \\
\text { desvert. , Sozialvers. }\end{array}$ \\
\hline 7513 & $\begin{array}{l}\text { Wirtschaftsförderung, - ord- } \\
\text { nung und - aufsicht }\end{array}$ & Öffentliche Verwaltung & $\begin{array}{l}\text { Öffentl. } \quad \text { Verwaltung, } \\
\text { Sozialvers. , Landesverteidi- } \\
\text { gung }\end{array}$ & $\begin{array}{l}\text { Öffentl. Verwaltung, Lan- } \\
\text { desvert. , Sozialvers. }\end{array}$ \\
\hline 7514 & $\begin{array}{l}\text { Sonst. m. d. öffentl. Ver- } \\
\text { waltung verbundene Tätigk. }\end{array}$ & Öffentliche Verwaltung & $\begin{array}{l}\text { Öffentl. Verwaltung, } \\
\text { Sozialvers. , Landesverteidi- } \\
\text { gung }\end{array}$ & $\begin{array}{l}\text { Öffentl. Verwaltung, Lan- } \\
\text { desvert. , Sozialvers. }\end{array}$ \\
\hline 7521 & Auswärtige Angelegenheiten & $\begin{array}{l}\text { Auswert. Angelegenh. } \\
\text { Landesverteidigung, Justiz }\end{array}$ & $\begin{array}{l}\text { Öffentl. Verwaltung, } \\
\text { Sozialvers. , Landesverteidi- } \\
\text { gung }\end{array}$ & $\begin{array}{l}\text { Öffentl. Verwaltung, Lan- } \\
\text { desvert. , Sozialvers. }\end{array}$ \\
\hline 7522 & Landesverteidigung & $\begin{array}{l}\text { Auswert. Angelegenh. , } \\
\text { Landesverteidigung, Justiz }\end{array}$ & $\begin{array}{l}\text { Öffentl. Verwaltung, } \\
\text { Sozialvers. , Landesverteidi- } \\
\text { gung }\end{array}$ & $\begin{array}{l}\text { Öffentl. Verwaltung, Lan- } \\
\text { desvert. , Sozialvers. }\end{array}$ \\
\hline 7523 & Justiz & $\begin{array}{l}\text { Auswert. Angelegenh. } \\
\text { Landesverteidigung, Justiz }\end{array}$ & $\begin{array}{l}\text { Öffentl. Verwaltung, } \\
\text { Sozialvers. , Landesverteidi- } \\
\text { gung }\end{array}$ & $\begin{array}{l}\text { Öffentl. Verwaltung, Lan- } \\
\text { desvert. , Sozialvers. }\end{array}$ \\
\hline 7524 & $\begin{array}{l}\text { Öffentliche Sicherheit und } \\
\text { Ordnung }\end{array}$ & $\begin{array}{l}\text { Auswert. Angelegenh. } \\
\text { Landesverteidigung, Justiz }\end{array}$ & $\begin{array}{l}\text { Öffentl. Verwaltung, } \\
\text { Sozialvers. , Landesverteidi- } \\
\text { gung }\end{array}$ & $\begin{array}{l}\text { Öffentl. Verwaltung, Lan- } \\
\text { desvert. , Sozialvers. }\end{array}$ \\
\hline 7525 & Feuerwehr & $\begin{array}{l}\text { Auswert. Angelegenh. } \\
\text { Landesverteidigung, Justiz }\end{array}$ & $\begin{array}{l}\text { Öffentl. Verwaltung, } \\
\text { Sozialvers. , Landesverteidi- } \\
\text { gung }\end{array}$ & $\begin{array}{l}\text { Öffentl. Verwaltung, Lan- } \\
\text { desvert. , Sozialvers. }\end{array}$ \\
\hline 7530 & Sozialversicherung & Sozialversicherung & $\begin{array}{l}\text { Öffentl. Verwaltung, } \\
\text { Sozialvers. , Landesverteidi- } \\
\text { gung }\end{array}$ & $\begin{array}{l}\text { Öffentl. Verwaltung, Lan- } \\
\text { desvert. , Sozialvers. }\end{array}$ \\
\hline 8010 & Kindergärten, Volksschulen & Kindergärten, Volksschulen & Unterrichtswesen & Unterrichtswesen \\
\hline 8021 & $\begin{array}{l}\text { Allgemeinbildende weit- } \\
\text { erführende Schulen }\end{array}$ & Weiterführende Schulen & Unterrichtswesen & Unterrichtswesen \\
\hline 8022 & $\begin{array}{l}\text { Berufsbildende } \\
\text { erführende Schulen }\end{array}$ & Weiterführende Schulen & Unterrichtswesen & Unterrichtswesen \\
\hline 8030 & Hochschulen & Hochschulen & Unterrichtswesen & Unterrichtswesen \\
\hline 8041 & Fahrschulen & $\begin{array}{l}\text { Erwachsenenbild. u. sonst. } \\
\text { Unterricht }\end{array}$ & Unterrichtswesen & Unterrichtswesen \\
\hline 8042 & $\begin{array}{l}\text { Erwachsenenbildung und } \\
\text { Unterricht a. n. g. }\end{array}$ & $\begin{array}{l}\text { Erwachsenenbild. u. sonst. } \\
\text { Unterricht }\end{array}$ & Unterrichtswesen & Unterrichtswesen \\
\hline 8511 & Krankenhäuser & Gesundheitswesen & $\begin{array}{l}\text { Gesundheits- , Veterinär- u. } \\
\text { Sozialwesen }\end{array}$ & $\begin{array}{l}\text { Gesundheits- , Veterinär- u. } \\
\text { Sozialwesen }\end{array}$ \\
\hline 8512 & $\begin{array}{l}\text { Arztpraxen (ohne Zahnarzt- } \\
\text { praxen) }\end{array}$ & Gesundheitswesen & $\begin{array}{l}\text { Gesundheits- , Veterinär- u. } \\
\text { Sozialwesen }\end{array}$ & $\begin{array}{l}\text { Gesundheits- , Veterinär- u. } \\
\text { Sozialwesen }\end{array}$ \\
\hline 8513 & Zahnarztpraxen & Gesundheitswesen & $\begin{array}{l}\text { Gesundheits- , Veterinär- u. } \\
\text { Sozialwesen }\end{array}$ & $\begin{array}{l}\text { Gesundheits- , Veterinär- u. } \\
\text { Sozialwesen }\end{array}$ \\
\hline 8514 & Gesundheitswesen a. n. g. & Gesundheitswesen & $\begin{array}{l}\text { Gesundheits- , Veterinär- u. } \\
\text { Sozialwesen }\end{array}$ & $\begin{array}{l}\text { Gesundheits- , Veterinär- u. } \\
\text { Sozialwesen }\end{array}$ \\
\hline 8520 & Veterinärwesen & Veterinärwesen & $\begin{array}{l}\text { Gesundheits- , Veterinär- u. } \\
\text { Sozialwesen }\end{array}$ & $\begin{array}{l}\text { Gesundheits- , Veterinär- u. } \\
\text { Sozialwesen }\end{array}$ \\
\hline 8531 & $\begin{array}{l}\text { Heime (o. Fremden- , } \\
\text { Erholungs- u. Ferienheime) }\end{array}$ & Sozialwesen & $\begin{array}{l}\text { Gesundheits- , Veterinär- u. } \\
\text { Sozialwesen }\end{array}$ & $\begin{array}{l}\text { Gesundheits- , Veterinär- u. } \\
\text { Sozialwesen }\end{array}$ \\
\hline 8532 & Sozialwesen a. n. g. & Sozialwesen & $\begin{array}{l}\text { Gesundheits- , Veterinär- u. } \\
\text { Sozialwesen }\end{array}$ & $\begin{array}{l}\text { Gesundheits- , Veterinär- u. } \\
\text { Sozialwesen }\end{array}$ \\
\hline 9000 & $\begin{array}{l}\text { Abwasser- u. Abfallbeseiti- } \\
\text { gung u. sonst. Entsorgung } \\
\text { (bis } 7 / 04 \text { ) }\end{array}$ & $\begin{array}{l}\text { Abwasser- u. Abfallbeseiti- } \\
\text { gung u. sonst. Entsorgung }\end{array}$ & $\begin{array}{l}\text { Abwasser- u. Abfallbeseiti- } \\
\text { gung u. sonst. Entsorgung }\end{array}$ & $\begin{array}{l}\text { Erbr. v. sonst. öffentl. u. } \\
\text { persönl. Dienstl. }\end{array}$ \\
\hline 9001 & Kanalisation u. Kläranlagen & $\begin{array}{l}\text { Abwasser- u. Abfallbeseiti- } \\
\text { gung u. sonst. Entsorgung }\end{array}$ & $\begin{array}{l}\text { Abwasser- u. Abfallbeseiti- } \\
\text { gung u. sonst. Entsorgung }\end{array}$ & $\begin{array}{l}\text { Erbr. v. sonst. öffentl. u. } \\
\text { persönl. Dienstl. }\end{array}$ \\
\hline 9002 & $\begin{array}{l}\text { Müllabfuhr, Mülldeponie, } \\
\text { Sondermüll }\end{array}$ & $\begin{array}{l}\text { Abwasser- u. Abfallbeseiti- } \\
\text { gung u. sonst. Entsorgung }\end{array}$ & $\begin{array}{l}\text { Abwasser- u. Abfallbeseiti- } \\
\text { gung u. sonst. Entsorgung }\end{array}$ & $\begin{array}{l}\text { Erbr. v. sonst. öffentl. u. } \\
\text { persönl. Dienstl. }\end{array}$ \\
\hline 9003 & Schneeräumung & $\begin{array}{l}\text { Abwasser- u. Abfallbeseiti- } \\
\text { gung u. sonst. Entsorgung }\end{array}$ & $\begin{array}{l}\text { Abwasser- u. Abfallbeseiti- } \\
\text { gung u. sonst. Entsorgung }\end{array}$ & $\begin{array}{l}\text { Erbr. v. sonst. öffentl. u. } \\
\text { persönl. Dienstl. }\end{array}$ \\
\hline
\end{tabular}

Continued on next page... 


\begin{tabular}{|c|c|c|c|c|}
\hline code & $\begin{array}{l}\text { industry } \\
\text { (very narrow) }\end{array}$ & $\begin{array}{l}\text { industry } \\
\text { (narrow) }\end{array}$ & $\begin{array}{l}\text { industry } \\
\text { (broad) }\end{array}$ & $\begin{array}{l}\text { industry } \\
\text { (very broad) }\end{array}$ \\
\hline 9111 & $\begin{array}{l}\text { Wirtschafts- und Arbeitge- } \\
\text { berverbände }\end{array}$ & $\begin{array}{l}\text { Wirtschafts- u. Arbeitge- } \\
\text { berverbände }\end{array}$ & $\begin{array}{l}\text { Interessensvertr. kirchl. u. } \\
\text { sonst. religiöse Vereine }\end{array}$ & $\begin{array}{l}\text { Erbr. v. sonst. öffentl. u. } \\
\text { persönl. Dienstl. }\end{array}$ \\
\hline 9112 & Berufsorganisationen & $\begin{array}{l}\text { Wirtschafts- u. Arbeitge- } \\
\text { berverbände }\end{array}$ & $\begin{array}{l}\text { Interessensvertr. kirchl. u. } \\
\text { sonst. religiöse Vereine }\end{array}$ & $\begin{array}{l}\text { Erbr. v. sonst. öffentl. u. } \\
\text { persönl. Dienstl. }\end{array}$ \\
\hline 9120 & Arbeitnehmervereinigungen & Arbeitnehmervereinigungen & $\begin{array}{l}\text { Interessensvertr. kirchl. u. } \\
\text { sonst. religiöse Vereine }\end{array}$ & $\begin{array}{l}\text { Erbr. v. sonst. öffentl. u. } \\
\text { persönl. Dienstl. }\end{array}$ \\
\hline 9131 & $\begin{array}{l}\text { Kirchliche u. sonst. religiöse } \\
\text { Vereinigungen }\end{array}$ & $\begin{array}{l}\text { Kirchl. u. sonst. relig. Vere- } \\
\text { inigungen, Parteien }\end{array}$ & $\begin{array}{l}\text { Interessensvertr. kirchl. u. } \\
\text { sonst. religiöse Vereine }\end{array}$ & $\begin{array}{l}\text { Erbr. v. sonst. öffentl. u. } \\
\text { persönl. Dienstl. }\end{array}$ \\
\hline 9132 & Politische Parteien & $\begin{array}{l}\text { Kirchl. u. sonst. relig. Vere- } \\
\text { inigungen, Parteien }\end{array}$ & $\begin{array}{l}\text { Interessensvertr. kirchl. u. } \\
\text { sonst. religiöse Vereine }\end{array}$ & $\begin{array}{l}\text { Erbr. v. sonst. öffentl. u. } \\
\text { persönl. Dienstl. }\end{array}$ \\
\hline 9133 & $\begin{array}{l}\text { Sonstige Interessenvertre- } \\
\text { tungen und Vereine }\end{array}$ & $\begin{array}{l}\text { Kirchl. u. sonst. relig. Vere- } \\
\text { inigungen, Parteien }\end{array}$ & $\begin{array}{l}\text { Interessensvertr. kirchl. u. } \\
\text { sonst. religiöse Vereine }\end{array}$ & $\begin{array}{l}\text { Erbr. v. sonst. öffentl. u. } \\
\text { persönl. Dienstl. }\end{array}$ \\
\hline 9211 & Film- und Videoherstellung & $\begin{array}{l}\text { Film- u. Videoherstellung, } \\
\text { Lichtspieltheater }\end{array}$ & $\begin{array}{l}\text { Kultur, Sport u. Unterhal- } \\
\text { tung }\end{array}$ & $\begin{array}{l}\text { Erbr. v. sonst. öffentl. u. } \\
\text { persönl. Dienstl. }\end{array}$ \\
\hline 9212 & $\begin{array}{l}\text { Filmverleih und Videopro- } \\
\text { grammanbieter }\end{array}$ & $\begin{array}{l}\text { Film- u. Videoherstellung, } \\
\text { Lichtspieltheater }\end{array}$ & $\begin{array}{l}\text { Kultur, Sport u. Unterhal- } \\
\text { tung }\end{array}$ & $\begin{array}{l}\text { Erbr. v. sonst. öffentl. u. } \\
\text { persönl. Dienstl. }\end{array}$ \\
\hline 9213 & Lichtspieltheater (Kinos) & $\begin{array}{l}\text { Film- u. Videoherstellung, } \\
\text { Lichtspieltheater }\end{array}$ & $\begin{array}{l}\text { Kultur, Sport u. Unterhal- } \\
\text { tung }\end{array}$ & $\begin{array}{l}\text { Erbr. v. sonst. öffentl. u. } \\
\text { persönl. Dienstl. }\end{array}$ \\
\hline 9220 & $\begin{array}{l}\text { Hörfunk- un Fernsehanstal- } \\
\text { ten }\end{array}$ & $\begin{array}{l}\text { Hörfunk- un Fernsehanstal- } \\
\text { ten }\end{array}$ & $\begin{array}{l}\text { Kultur, Sport u. Unterhal- } \\
\text { tung }\end{array}$ & $\begin{array}{l}\text { Erbr. v. sonst. öffentl. u. } \\
\text { persönl. Dienstl. }\end{array}$ \\
\hline 9231 & $\begin{array}{l}\text { Künstlerische u. schriftstel- } \\
\text { lerische Tätigkeiten }\end{array}$ & $\begin{array}{l}\text { Erbr. v. sonst. kulturellen } \\
\text { Leistungen }\end{array}$ & $\begin{array}{l}\text { Kultur, Sport u. Unterhal- } \\
\text { tung }\end{array}$ & $\begin{array}{l}\text { Erbr. v. sonst. öffentl. u. } \\
\text { persönl. Dienstl. }\end{array}$ \\
\hline 9232 & $\begin{array}{l}\text { Betrieb u. techn. Hilfsdien- } \\
\text { ste f. d. Unterhaltung }\end{array}$ & $\begin{array}{l}\text { Erbr. v. sonst. kulturellen } \\
\text { Leistungen }\end{array}$ & $\begin{array}{l}\text { Kultur, Sport u. Unterhal- } \\
\text { tung }\end{array}$ & $\begin{array}{l}\text { Erbr. v. sonst. öffentl. u. } \\
\text { persönl. Dienstl. }\end{array}$ \\
\hline 9233 & $\begin{array}{l}\text { Schaustellergewerbe und } \\
\text { Vergnügungsparks }\end{array}$ & $\begin{array}{l}\text { Erbr. v. sonst. kulturellen } \\
\text { Leistungen }\end{array}$ & $\begin{array}{l}\text { Kultur, Sport u. Unterhal- } \\
\text { tung }\end{array}$ & $\begin{array}{l}\text { Erbr. v. sonst. öffentl. u. } \\
\text { persönl. Dienstl. }\end{array}$ \\
\hline 9234 & $\begin{array}{l}\text { Erbr. v. kulturellen u. un- } \\
\text { terhaltenden Leistungen }\end{array}$ & $\begin{array}{l}\text { Erbr. v. sonst. kulturellen } \\
\text { Leistungen }\end{array}$ & $\begin{array}{l}\text { Kultur, Sport u. Unterhal- } \\
\text { tung }\end{array}$ & $\begin{array}{l}\text { Erbr. v. sonst. öffentl. u. } \\
\text { persönl. Dienstl. }\end{array}$ \\
\hline 9240 & $\begin{array}{l}\text { Korrespondenz- } \\
\text { Nachrichtenbüros }\end{array}$ & $\begin{array}{l}\text { Korrespondenz- } \\
\text { Nachrichtenbüros }\end{array}$ & $\begin{array}{l}\text { Kultur, Sport u. Unterhal- } \\
\text { tung }\end{array}$ & $\begin{array}{l}\text { Erbr. v. sonst. öffentl. u. } \\
\text { persönl. Dienstl. }\end{array}$ \\
\hline 9251 & Bibliotheken und Archive & $\begin{array}{l}\text { Bibliotheken, Musees, bot. } \\
\text { u. zool. Gärten }\end{array}$ & $\begin{array}{l}\text { Kultur, Sport u. Unterhal- } \\
\text { tung }\end{array}$ & $\begin{array}{l}\text { Erbr. v. sonst. öffentl. u. } \\
\text { persönl. Dienstl. }\end{array}$ \\
\hline 9252 & Museen und Denkmalschutz & $\begin{array}{l}\text { Bibliotheken, Musees, bot. } \\
\text { u. zool. Gärten }\end{array}$ & $\begin{array}{l}\text { Kultur, Sport u. Unterhal- } \\
\text { tung }\end{array}$ & $\begin{array}{l}\text { Erbr. v. sonst. öffentl. u. } \\
\text { persönl. Dienstl. }\end{array}$ \\
\hline 9253 & $\begin{array}{l}\text { Botanische u. zoologische } \\
\text { Gärten, Naturparks }\end{array}$ & $\begin{array}{l}\text { Bibliotheken, Musees, bot. } \\
\text { u. zool. Gärten }\end{array}$ & $\begin{array}{l}\text { Kultur, Sport u. Unterhal- } \\
\text { tung }\end{array}$ & $\begin{array}{l}\text { Erbr. v. sonst. öffentl. u. } \\
\text { persönl. Dienstl. }\end{array}$ \\
\hline 9261 & Betrieb von Sportanlagen & Sport & $\begin{array}{l}\text { Kultur, Sport u. Unterhal- } \\
\text { tung }\end{array}$ & $\begin{array}{l}\text { Erbr. v. sonst. öffentl. u. } \\
\text { persönl. Dienstl. }\end{array}$ \\
\hline 9262 & $\begin{array}{l}\text { Erbr. v. sonst. Dienstleis- } \\
\text { tungen d. Sports }\end{array}$ & Sport & $\begin{array}{l}\text { Kultur, Sport u. Unterhal- } \\
\text { tung }\end{array}$ & $\begin{array}{l}\text { Erbr. v. sonst. öffentl. u. } \\
\text { persönl. Dienstl. }\end{array}$ \\
\hline 9271 & $\begin{array}{l}\text { Spiel- , Wett- und Lot- } \\
\text { teriewesen }\end{array}$ & $\begin{array}{l}\text { Erbr. v. sonst. Dienstl. f. } \\
\text { Unterhaltung u. Freizeit }\end{array}$ & $\begin{array}{l}\text { Kultur, Sport u. Unterhal- } \\
\text { tung }\end{array}$ & $\begin{array}{l}\text { Erbr. v. sonst. öffentl. u. } \\
\text { persönl. Dienstl. }\end{array}$ \\
\hline 9272 & $\begin{array}{l}\text { Erbr. v. sonst. Dienstl. f. } \\
\text { Unterhaltung u. Freizeit }\end{array}$ & $\begin{array}{l}\text { Erbr. v. sonst. Dienstl. f. } \\
\text { Unterhaltung u. Freizeit }\end{array}$ & $\begin{array}{l}\text { Kultur, Sport u. Unterhal- } \\
\text { tung }\end{array}$ & $\begin{array}{l}\text { Erbr. v. sonst. öffentl. u. } \\
\text { persönl. Dienstl. }\end{array}$ \\
\hline 9301 & $\begin{array}{l}\text { Wäscherei und chemische } \\
\text { Reinigung }\end{array}$ & $\begin{array}{l}\text { Erbringung v. sonst. Dien- } \\
\text { stleistungen }\end{array}$ & $\begin{array}{l}\text { Erbringung v. sonst. Dien- } \\
\text { stleistungen }\end{array}$ & $\begin{array}{l}\text { Erbr. v. sonst. öffentl. u. } \\
\text { persönl. Dienstl. }\end{array}$ \\
\hline 9302 & Kosmetiker, & $\begin{array}{l}\text { Erbringung v. sonst. Dien- } \\
\text { stleistungen }\end{array}$ & $\begin{array}{l}\text { Erbringung v. sonst. Dien- } \\
\text { stleistungen }\end{array}$ & $\begin{array}{l}\text { Erbr. v. sonst. öffentl. u. } \\
\text { persönl. Dienstl. }\end{array}$ \\
\hline 9303 & Bestattungswesen & $\begin{array}{l}\text { Erbringung v. sonst. Dien- } \\
\text { stleistungen }\end{array}$ & $\begin{array}{l}\text { Erbringung v. sonst. Dien- } \\
\text { stleistungen }\end{array}$ & $\begin{array}{l}\text { Erbr. v. sonst. öffentl. u. } \\
\text { persönl. Dienstl. }\end{array}$ \\
\hline 9304 & Bäder, Saunas, Solarien u. ä. & $\begin{array}{l}\text { Erbringung v. sonst. Dien- } \\
\text { stleistungen }\end{array}$ & $\begin{array}{l}\text { Erbringung v. sonst. Dien- } \\
\text { stleistungen }\end{array}$ & $\begin{array}{l}\text { Erbr. v. sonst. öffentl. u. } \\
\text { persönl. Dienstl. }\end{array}$ \\
\hline 9305 & $\begin{array}{l}\text { Erbringung von Dienstleis- } \\
\text { tungen a. n. g. }\end{array}$ & $\begin{array}{l}\text { Erbringung v. sonst. Dien- } \\
\text { stleistungen }\end{array}$ & $\begin{array}{l}\text { Erbringung v. sonst. Dien- } \\
\text { stleistungen }\end{array}$ & $\begin{array}{l}\text { Erbr. v. sonst. öffentl. u. } \\
\text { persönl. Dienstl. }\end{array}$ \\
\hline 9500 & Private Haushalte & Private Haushalte & Private Haushalte & Private Haushalte \\
\hline 9600 & $\begin{array}{l}\text { Herst. v. Waren d. priv. } \\
\text { Haushalte f. d. Eigenbedarf }\end{array}$ & $\begin{array}{l}\text { Herst. v. Waren d. priv. } \\
\text { Haushalte f. d. Eigenbedarf }\end{array}$ & $\begin{array}{l}\text { Herst. v. Waren d. priv. } \\
\text { Haushalte f. d. Eigenbedarf }\end{array}$ & $\begin{array}{l}\text { Herst. v. Waren d. priv. } \\
\text { Haushalte f. d. Eigenbedarf }\end{array}$ \\
\hline 9700 & $\begin{array}{l}\text { Erbring. v. Dienstl. d. priv. } \\
\text { Haushalte f. d. Eigenbedarf }\end{array}$ & $\begin{array}{l}\text { Erbring. v. Dienstl. d. priv. } \\
\text { Haushalte f. d. Eigenbedarf }\end{array}$ & $\begin{array}{l}\text { Erbring. v. Dienstl. d. priv. } \\
\text { Haushalte f. d. Eigenbedarf }\end{array}$ & $\begin{array}{l}\text { Erbring. v. Dienstl. d. priv. } \\
\text { Haushalte f. d. Eigenbedarf }\end{array}$ \\
\hline 9900 & $\begin{array}{l}\text { Exterritoriale Organis. u. } \\
\text { Körperschaften }\end{array}$ & $\begin{array}{l}\text { Exterritoriale Organis. u. } \\
\text { Körperschaften }\end{array}$ & $\begin{array}{l}\text { Exterritoriale Organisat. u. } \\
\text { Körperschaften }\end{array}$ & $\begin{array}{l}\text { Exterritoriale Organisat. u. } \\
\text { Körperschaften }\end{array}$ \\
\hline
\end{tabular}


Table 13: Codelist for the variable onace_17

\begin{tabular}{|c|c|}
\hline code & industry \\
\hline 1 & Land- und Forstwirtschaft \\
\hline 2 & Fischerei und Fischzucht \\
\hline 3 & Bergbau und Gewinnung von Steinen und Erden \\
\hline 4 & Sachguetererzeugung \\
\hline 5 & Energie- und Wasserversorgung \\
\hline 6 & Bauwesen \\
\hline 7 & $\begin{array}{l}\text { Handel, Instandhaltung und Reparatur von Kraftfahrzeugen und Gebrauchs- } \\
\text { guetern }\end{array}$ \\
\hline 8 & Beherbergungs- und Gaststaettenwesen \\
\hline 9 & Verkehr und Nachrichtenuebermittlung \\
\hline 10 & Kredit- und Versicherungswesen \\
\hline 11 & $\begin{array}{l}\text { Realitaetenwesen, Vermietung beweglicher Sachen, Erbringung von unternehmen- } \\
\text { bezogenen Dienstleistungen }\end{array}$ \\
\hline 12 & Oeffentliche Verwaltung, Landesverteidigung, Sozialversicherung \\
\hline 13 & Unterrichtswesen \\
\hline 14 & Gesundheits-, Veterinaer- und Sozialwesen \\
\hline 15 & Erbringung von sonstigen oeffentlichen und persoenlichen Dienstleistungen \\
\hline 16 & Private Haushalte \\
\hline 17 & Exterritoriale Organisationen und Koerperschaften \\
\hline
\end{tabular}


Table 14: Codelist for the variable onace_60

\begin{tabular}{|c|c|}
\hline code & industry \\
\hline 1 & Landwirtschaft, Jagd \\
\hline 2 & Forstwirtschaft \\
\hline 5 & Fischerei und Fischzucht \\
\hline 10 & Kohlenbergbau, Torfgewinnung \\
\hline 11 & Erdöl- und Erdgasbergbau, sowie damit verbundene Dienstleistungen \\
\hline 12 & Bergbau auf Uran- und Thoriumerze \\
\hline 13 & Erzbergbau \\
\hline 14 & Gewinnung von Steinen und Erden, sonstiger Bergbau \\
\hline 15 & Herstellung von Nahrungs- und Genussmitteln und Getränken \\
\hline 16 & Tabakverarbeitung \\
\hline 17 & Herstellung von Textilien und Textilwaren (ohne Bekleidung) \\
\hline 18 & Herstellung von Bekleidung \\
\hline 19 & Ledererzeugung und -verarbeitung \\
\hline 20 & Be- und Verarbeitung von Holz (ohne Herstellung von Möbeln) \\
\hline 21 & Herstellung und Verarbeitung von Papier und Pappe \\
\hline 22 & $\begin{array}{l}\text { Verlagswesen, Druckerei, Vervielfältigung von bespielten Ton-, Bild-, und Da- } \\
\text { tenträgern }\end{array}$ \\
\hline 23 & $\begin{array}{l}\text { Kokerei, Mineralölverarbeitung, Herstellung und Verarbeitung von Spalt- und } \\
\text { Brutstoffen }\end{array}$ \\
\hline 24 & Herstellung von Chemikalien und chemischen Erzeugn \\
\hline 25 & Herstellung von Gummi- und Kunststoffwaren \\
\hline 26 & $\begin{array}{l}\text { Herstellung und Bearbeitung von Glas, Herstellung von Waren aus Steinen und } \\
\text { Erden }\end{array}$ \\
\hline 27 & Metallerzeugung und -bearbeitung \\
\hline 28 & Herstellung von Metallerzeugnissen \\
\hline 29 & Maschinenbau \\
\hline 30 & Herstellung von Büromaschinen, Datenverarbeitungsgeräten und -einrichtungen \\
\hline 31 & Herstellung von Geräten der Elektrizitätserzeugung, -verteilung u.f. \\
\hline 32 & Rundfunk-, Fernseh- und Nachrichtentechnik \\
\hline 33 & Medizin-, Mess-, Steuer- und Regelungstechnik, Optik \\
\hline 34 & Herstellung von Kraftwagen und Kraftwagenteilen \\
\hline 35 & Sonstiger Fahrzeugbau \\
\hline 36 & $\begin{array}{l}\text { Herstellung von Möbeln, Schmuck, Musikinstrumenten, Sportgeräten, Spielwaren } \\
\text { und sonstigen Erzeugnissen; Recycling }\end{array}$ \\
\hline 37 & Rückgewinnung (Recycling) \\
\hline 40 & Energieversorgung \\
\hline 41 & Wasserversorgung \\
\hline 45 & Bauwesen \\
\hline 50 & $\begin{array}{l}\text { Kraftfahrzeughandel, Instandhaltung und Reparatur von Kraftfahrzeugen, } \\
\text { Tankstellen }\end{array}$ \\
\hline 51 & Handelsvermittlung und Grosshandel (ohne Handel mit Kraftfahrzeugen) \\
\hline 52 & Einzelhandel (ohne Handel mit Kraftfahrzeugen und ohne Tankstellen) \\
\hline 55 & Beherbergungs- und Gaststättenwesen \\
\hline 60 & Landverkehr, Transport in Rohrfernleitungen \\
\hline 61 & Schiffahrt \\
\hline
\end{tabular}

Continued on next page... 


\begin{tabular}{|c|c|}
\hline code & industry \\
\hline 62 & Flugverkehr \\
\hline 63 & Hilfs- und Nebentätigkeiten für den Verkehr, Reisebüros \\
\hline 64 & Nachrichtenübermittlung \\
\hline 65 & Kreditwesen \\
\hline 66 & Versicherungswesen \\
\hline 67 & Mit dem Kredit- und Versicherungswesen verbundene Tätigkeiten \\
\hline 70 & Realitätenwesen \\
\hline 71 & Vermietung beweglicher Sachen ohne Bedienungspersonal \\
\hline 72 & Datenverarbeitung und Datenbanken \\
\hline 73 & Forschung und Entwicklung \\
\hline 74 & Erbringung von unternehmensbezogenen Dienstleistungen \\
\hline 75 & Öffentliche Verwaltung, Landesverteidigung, Sozialversicherung \\
\hline 80 & Unterrichtswesen \\
\hline 85 & Gesundheits-, Veterinär- und Sozialwesen \\
\hline 90 & Abwasser- und Abfallbeseitigung und sonstige Entsorgung \\
\hline 91 & Interessenvertretungen, kirchliche und sonstige religiöse Vereinigungen \\
\hline 92 & Kultur, Sport und Unterhaltung \\
\hline 93 & Erbringung von sonstigen Dienstleistungen \\
\hline 95 & Private Haushalte \\
\hline 99 & Exterritoriale Organisationen und Körperschaften \\
\hline
\end{tabular}

